A COMPILATION OF DATA ON FLUIDS FROM GEOTHERMAL RESOURCES AI THE

UNITED STATES 


\section{DISCLAIMER}

This report was prepared as an account of work sponsored by an agency of the United States Government. Neither the United States Government nor any agency Thereof, nor any of their employees, makes any warranty, express or implied, or assumes any legal liability or responsibility for the accuracy, completeness, or usefulness of any information, apparatus, product, or process disclosed, or represents that its use would not infringe privately owned rights. Reference herein to any specific commercial product, process, or service by trade name, trademark, manufacturer, or otherwise does not necessarily constitute or imply its endorsement, recommendation, or favoring by the United States Government or any agency thereof. The views and opinions of authors expressed herein do not necessarily state or reflect those of the United States Government or any agency thereof. 


\section{DISCLAIMER}

Portions of this document may be illegible in electronic image products. Images are produced from the best available original document. 


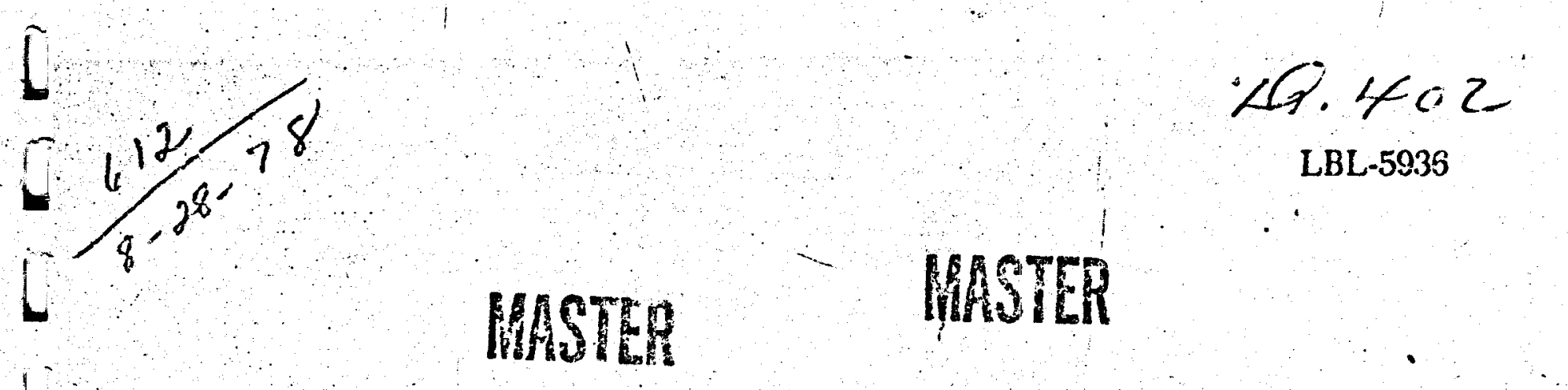

\title{
A Compilation of Data on Fluids from Geothermal Resources in the United States
}

\author{
S. R. Cosner and J. A. Apps
}

May 1978

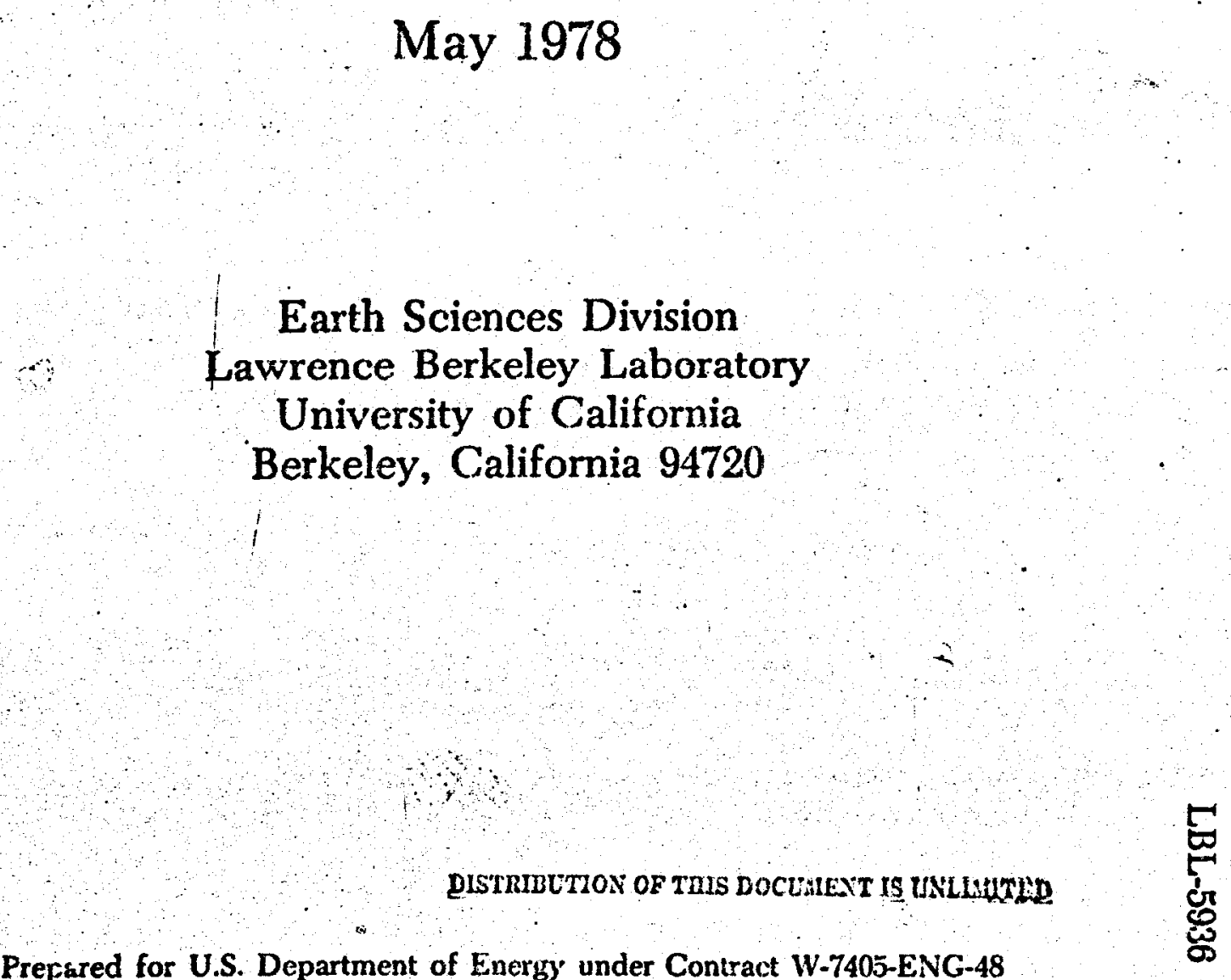

s

i)

Prepared for U.S. Department of Energy under Contract W-7405-ENG-48

REPROOUEED FMAMA DEST 


\section{TABLE OF CONTENTS}

\section{Page}

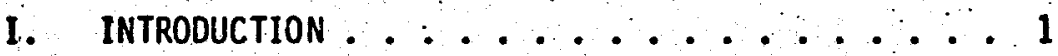

A. Background .............. 1

B. Scope of the Compilation. . . . ... 2

1. Resources Selected ......... 2

2. Information Compiled ...... 3

c. Computer Processing ......... 3

1. Storage of and Access to the Data. - 3

2. Bibliography ........ 7

References ............ 8

II. COMPILATION OF GEOTHERMAL FLUIO DATA .... 9

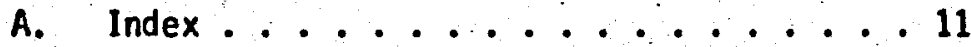

B. Compilation ................ 13

III. BIBLIOGRAPHY AND RELATED REFERENCES. . . . 95 
A COMPILATION OF DATA ON FLUIOOS FROM GEOTHERMAL RESOURCES IN THE UNITED STATES

S. R. Cosner and J. A. Apps.

\section{INTRODUCTION}

\section{A. Background}

The comercial developnent of geothermal energy for power and heating is receiving serious attention in the United States. Many resources are being investigated intensively, and the design and testing of geothermal systems and components is under way, both in government laboratories and in the private sector.

Geothermal energy can be extracted from resources containing native stean, hot water, geopressured water, hot dry rock, and magma. The most important resources in terms of immediate development potential are those containing hot water. Such resources are generally known as "liquid dominated," in contrast to "vapor dominated" or native steam sources.

Liquid dominated resources vary substantially in size, temperature, and fluid composition. The fluid composition can lead to many technical problems when such resources are exploited, including scaling, sludge formation, corrosion, and erosion in suriace facilities; the disposal of noncondensable gases; the treatment of toxic gases, volatiles and precipitates; and the disposal of spent fluids. Geothermal fluids also have differing thermodynamic and transport properties from pure water.. and these must be taken into account during geothermal power plant design. A knowledge of geothermal fluid characteristics is therefore important in anticipating such problems.

The purpose of this compilation is to provide information on the chemistry of geothermal fluids to scientists and engineers involved with the development of liquid dominated geothermal energy resources. The compilation is a comprehensive tabulation of available yeothermal fluid data from the most important geothermal resources in the United States. 
1. The compilation was funded by the Division of Geothermal Energy of the Department of Energy.

\section{B. Scope of the Compilation}

1. Resources Selected

The rescurces selected for this compilation were determined primarily from White and Williams. 1 Liquid dominated resources, with a heat capacity exceeding $1 \times 10^{18} \mathrm{cal}$ and temperatures graater than $90^{\circ} \mathrm{C}$, were candidates for inclusion in the data compilation. (Yellowstone National Park, however, was onitted because exploitation of the geothermal resources in national parks is prohibited.) Thirty-four candidates were identified in White and Williams. 1 In addition, resources at Cerro Prieto in Mexico, and Puna in Hawaii were included. The former was added because of its relevance to other geothermal resources in the Imperial Valley, California, and the latter because of significant drilling results obtained in 1977.

The resources chosen were those most likely to be exploited for geothermal energy during the next ten years. Particular importance was attached to their potential for electric power generation.

Only information on the chemical composition of fluids from wells was compiled. Hot spring analyses were not collected because such analyses are not considered to be representative of geothermal fluids found at depth within a geothermal reservoir. Furthermore, the U.S. Geological Survey is conducting a separate program to collect data on hot springs in wie United States. 2

Data from 17 resources were obtained by searching the literature as far back as 1929, and by collecting data from companies working in the geothermal energy field. Much of the data held by the private sector remains confidential because of the need to protect corporate investments in a promising geothermal area. For this reason information is lacking from wells drilled recently in such locations as Valles Caldera KGRA, New Mexico (Baca location No.. 1): Brawley KGRA, Imperial County, California; and Roosevelt Hot Springs KGRA, Beaver County, Utah. 


\section{Information Compiled}

A complete listing of the kinds of information that could possibly be compiled for each sample is shown in Table 1 . Not all information, however, could be fiund for each sample analysis. All information is recorded as found in the source document. No attempt, for example, was made to standardize units of measurement for the concentrations of components in solution.

Caution must be exercised in using the data in this compilation. The data come from a large variety of sources and are of variable quality. Users should consider the type of sample, the way it was obtained, the completeness of the analysis, the analytical methods, and the units of measurement used for reporting the results. Some samples may have been analyzed long after the sampling, leading to interim oxidation, precipitation, or evaporation of some of the constituents.

It should be recognized that geothermal fluids are rarely, if ever, uniform in composition. The analys is given for a particular geothermal well may not be representative of the resource as a whole. It may be little more than a reflection of unspecified mixtures of fluids or differing compositions from different horizons within a well. The composition of geathermal fluids can also change with time. Compositional fluctuations may occur in a flowing well during time periods as short as one hour.

\section{Computer Processing}

1. Storage of and Access to the Data

The compilation of geothermal fluid data has been processed and stored at Lawrence Berkeley Laboratory, using the Berkeley Data Base Management System (BDMS). This system is a generalized information retrieval system offering a broad range of capabilities for creating, maintaining, and accessing computer data bases. BDMA consists of an easy-to-use data base definition language, an editor that stores, modifies, and retrieves data, and a systcin that searches the data base for indices specified by the user. 3 


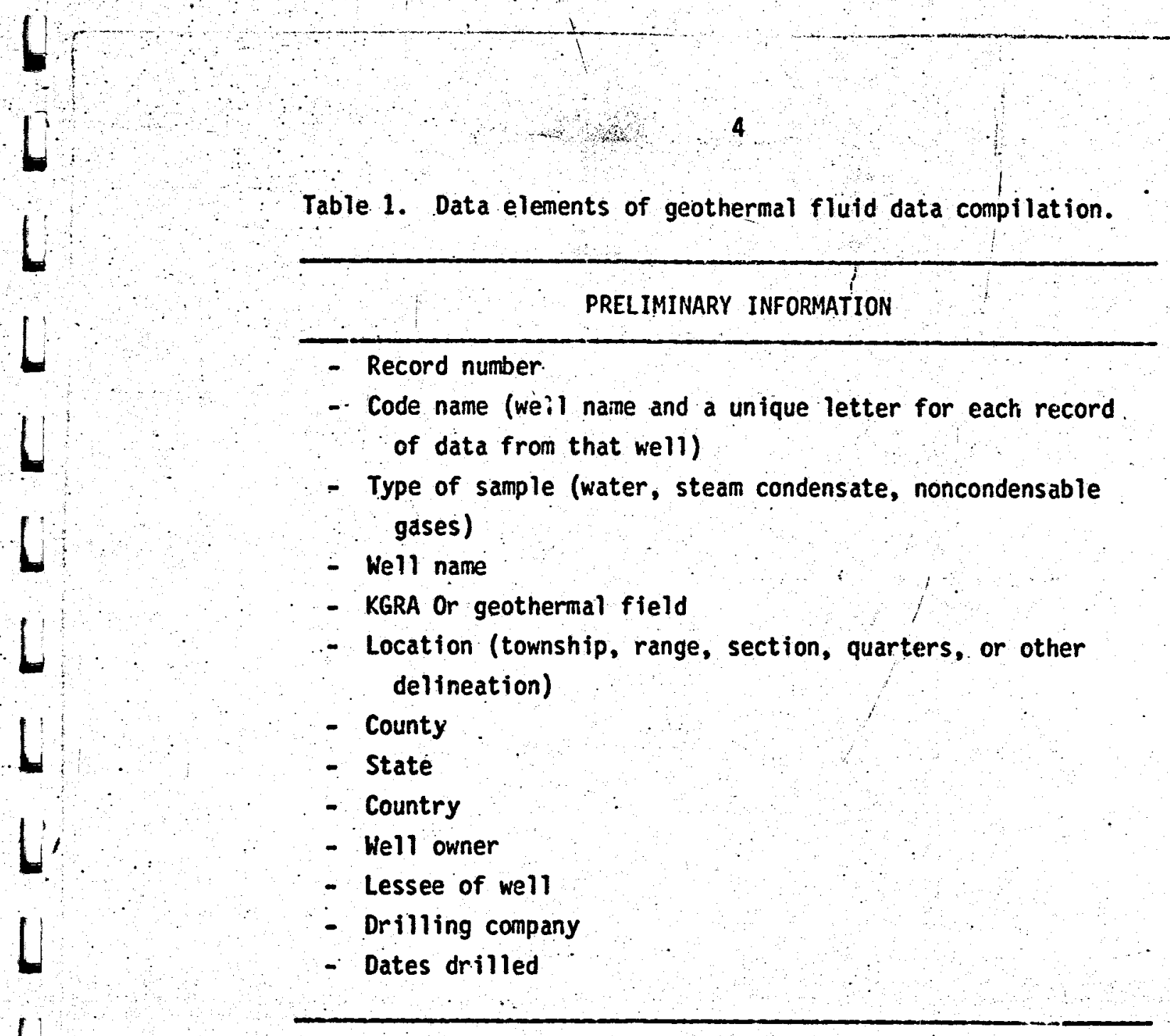

WELL DATA

- Well depth (in meters)

- Temperatures (in degrees Celsius)

- Depth or location of temperature reading

- Shut-in pressure

- Flow information (flow rates and pressures)

- Well casing preforation intervar

- Lithology of production zone 


\section{5}

Table 1\% Data elements of geothermal fluid data compilation (continued).

\section{SAMPLING INFORMATION}

- Date sample taken

- Sample number, analyzing laboratory

- Location sample taken

- Sampiing method

- Condition of sample when taken (temperature, pressure, whether fluid lost due to steam flashing)

- Condition of well when sample taken (flow time before sampling, flow rate, etc.)

PHYSICAL DATA

- $\mathrm{pH}, \mathrm{pH}$ range if given, temperature of reading

Eh, temperature of reading

- Specific gravity, temperature of reading

- Viscosity of fluid

- Total dissolved solids, whether sum of analysis or residue on evaporation

- Total alkalinity

- Other data

\section{BRINE DATA}

- Methods of analysis

- Error limits

- Units

- Constituent name, concentration, coment

- Comment, if needed, on table of analyses 
Table 1. Data elements of geothermal fluid data compilation (continued).

BIBLIOGRAPHIC DATAA

- Sources of data in record. Entry corresponds to the principal author's last name and year article was published

\section{OTHER NOTES}

- Other important information 
The compiled geothermal fluid data are stored in the data base as records. Within each record, data are stored under data elements defined by the data base user. Over 50 elements were defined for use in the geothermal fluid data compilation as shown in Table 1. Few records contain all of the data elements because the information compiled was usually incomplete.

Each record documents a specific sample analysis from one well. In those cases where there is more than one analys is for a well, there are multiple records for the wel1. An attempt was made to place the most reliable analysis in the first record.

The complete compilation, obta ined through a computer program written specifically for this purpose, follows in Section II of this report. Because the compilation is stored on the LBL computer system, it may be accessed and manipulated by users via a computer terminal. With special arrangements a user may connect to the LBL computer terminal. This connection also allows the user to search selectively for specific key subjects, and to print only those records of interest.

A more complicated operation using compilation through computer access involves the manipulation of the data in userwritten programs. This could be done, for example, using the data as input for a program modeling thermodynamic equilibria of a geothermal system. Programs could also be written to make statistical comparisons of various parameters stored in the compilation.

\section{Bibliography}

The bibliography given in Section III is a listing of all the sources from which data were collected for the compilation. The bibliography, like the geothermal fluid data compllation, was input to the BDMS computer to facilitate editing and retrieval on specified subjects. The format used is the same as that used by the National Geothermal Information Resource Group (GRID) at LBL. 2,4 The descriptors for each reference are key words, taken from a standard thesaurus, 5 which describe the subject 
matter discussed in the report or article. These key words facilitate computer searches for specific subjects.

The name and number in the upper right section of each bibliographic listing is made up of the principal author's last name and the year the article was published. This entry corresponds to that listed as the source of data at the end of each record in the fluic data compilation.

The bibliography contains listings of some reports and articles that were not used as sources in the compilation. They were scanned for data, however, and are included in the bibliography because they may be useful to investigators.

\section{References}

1. White, D. E. and Williams, D. L. Assessment of Geotherma1 Resources of the United States, U.S.G.S. Circular 726 (1975). 2. Clark, A. L., Calkins, J. A., Tongiorgi, E., and Stefanelli, E. A Report on the International Geothermal Information Exchange. Program, 1974-1975, in Proceedings-Second United Nations Symposium on the Development and Use of Geothermal Resources, Lawrence Berkeley Laboratory, vol. 1, pp. 67-100 (1976).

3. Richards, D. R. BDMS - Berkeley Data Base Management System

User's Manual, Lawrence Berkeley Laboratory, Report LBL-4683 (April 1976, revised 1977).

4. Trippe, T., White, W., Henderson, F., and Phillips, S. L., GEODOC - The GRID Document File, Record Structure and Data Element Description, Lawrence Berkeley Laboratnry. Report LBL-4432 (6 November 1975).

5. Perra, J. J. and Herr, J. J. Geothermal Thesaurus, Lawrence Berkeley Laboratory, Report LBL-4841, draft (June 1977). 



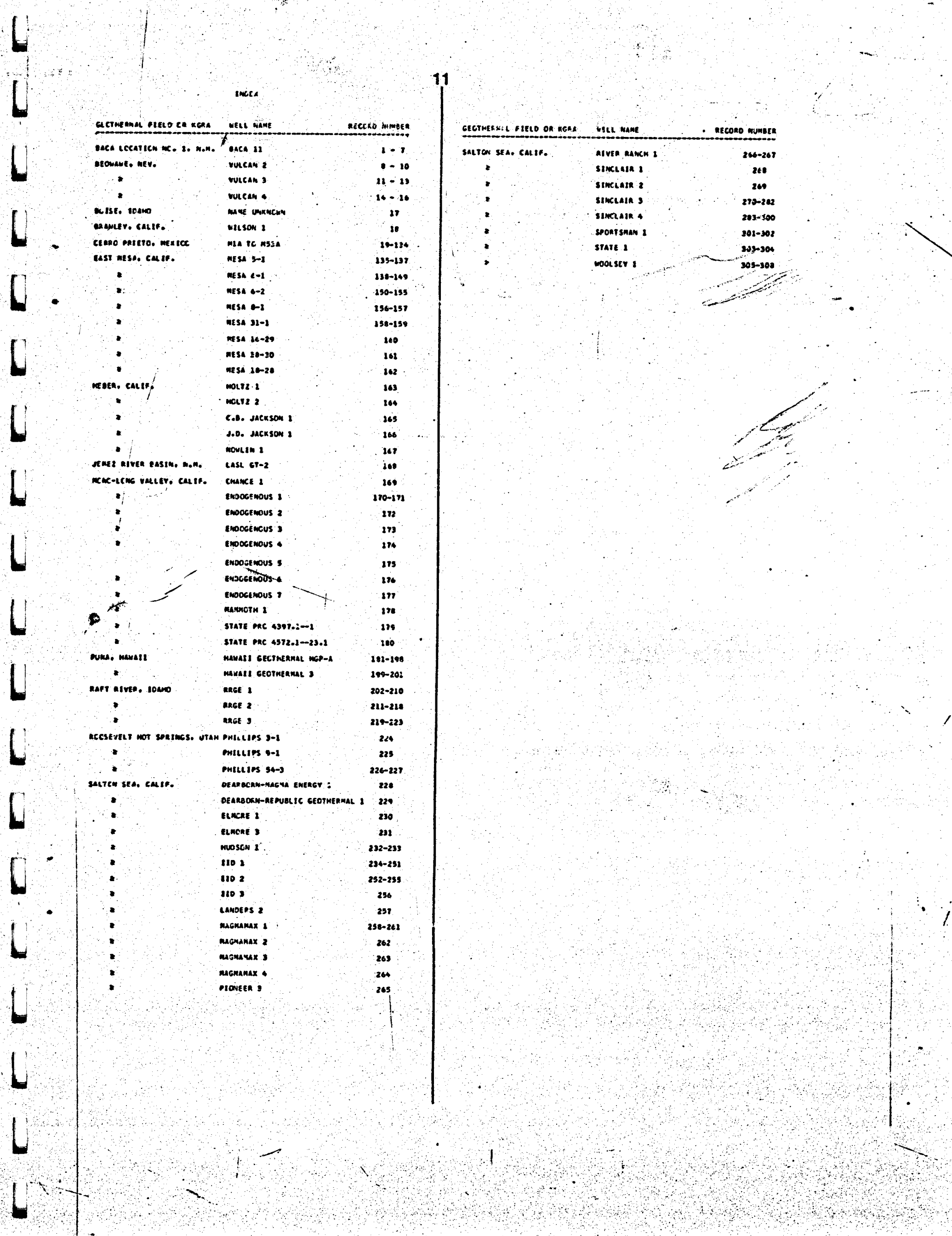



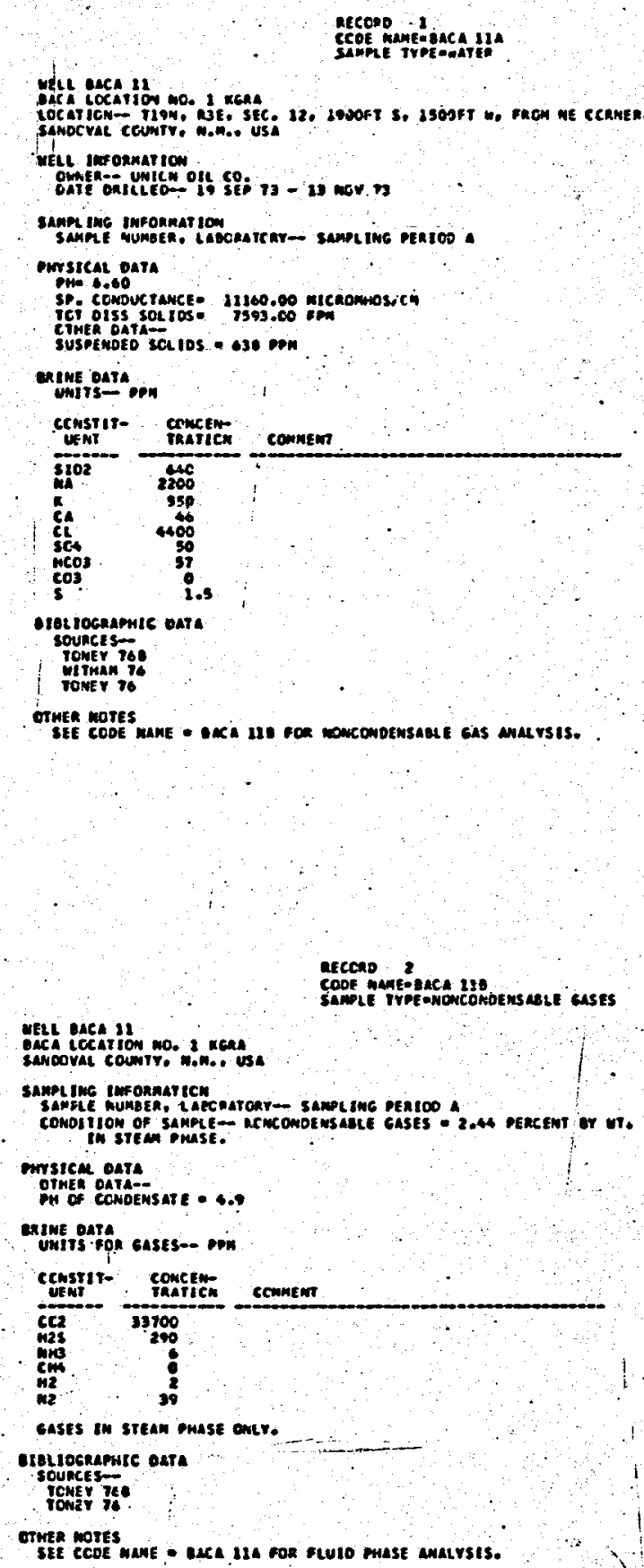


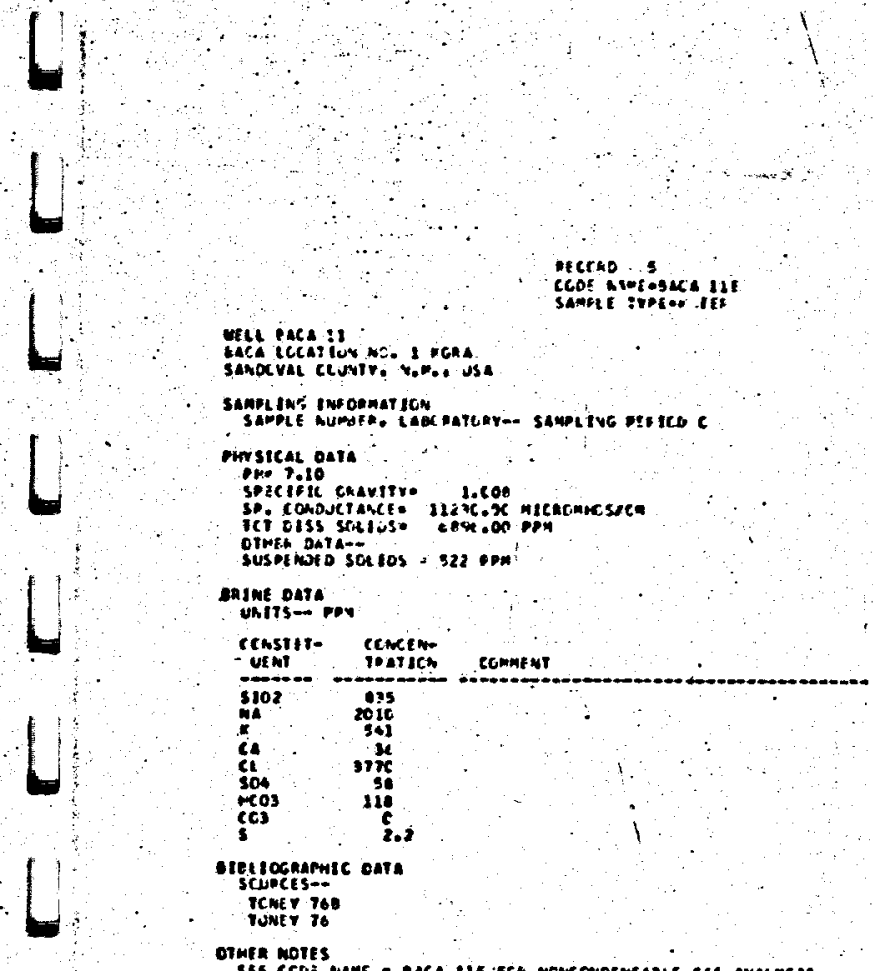

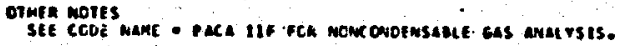

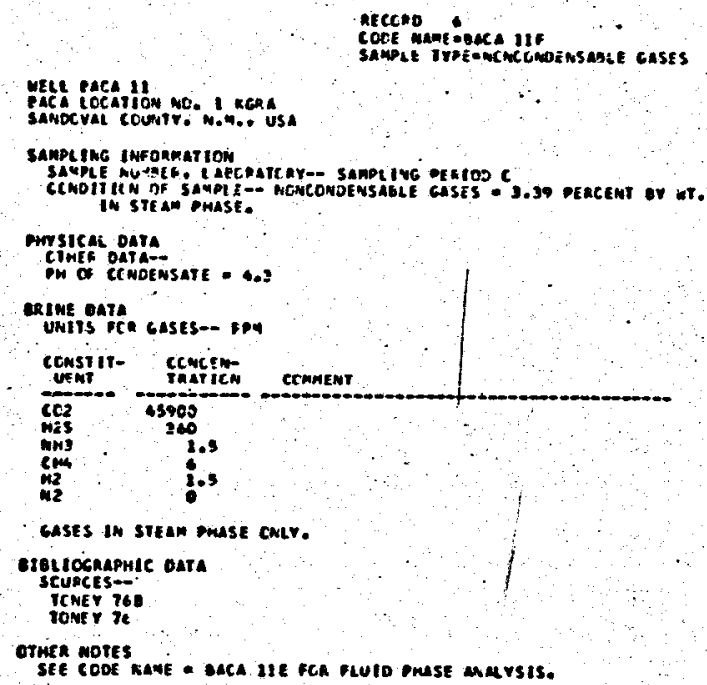

Hite ace 13

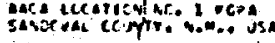

semping isfopmention

myscet eati.

pon cl crbinsute -4.7

eneste onle

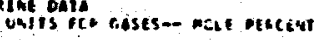

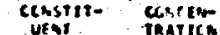

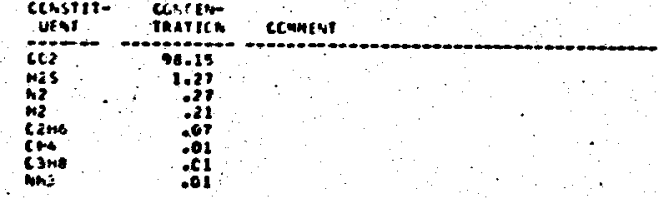

cases in ștean buase crity.

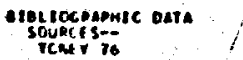

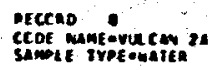

MELL Nican 2

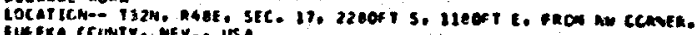

well enscomation

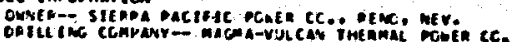

mete oara

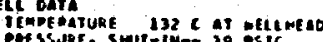

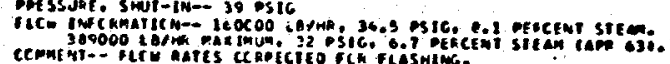

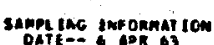

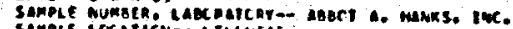

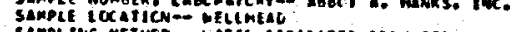

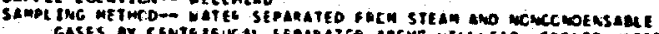

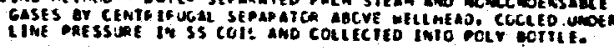

omrsical oatra

PS. S.30 TCi otss sol los: \$35.00 PPM, EVAPCEATEO A1 105 C

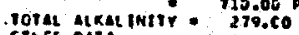

Chit cata-ing a 270 op:

- $123 \mathrm{mon}$

exting parts.ron

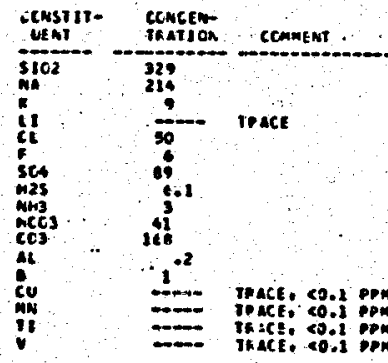

igziocrephic Dats

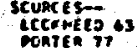

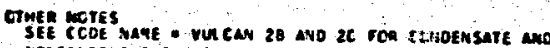

wCacr wo nsARLe gas avalYSES. 


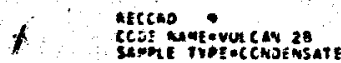

vect vutcan 2

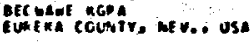

sant ing Imsonmartice

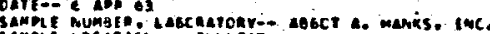

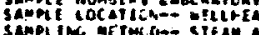

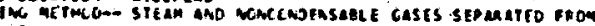

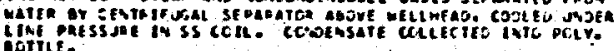

insical dara

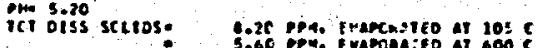

iemet oata-.-

clotir to on e.2 as caces - $30 \mathrm{rom}$

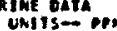

CCAsili- CCMCE

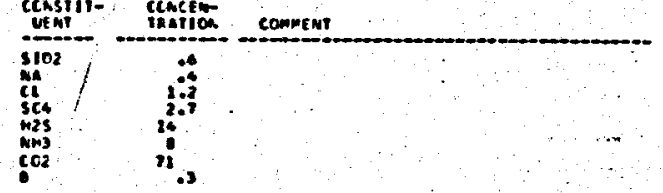

eres locrapuic data

cockeseo os

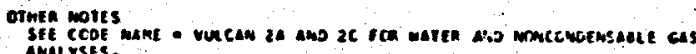

Sftectrok

1
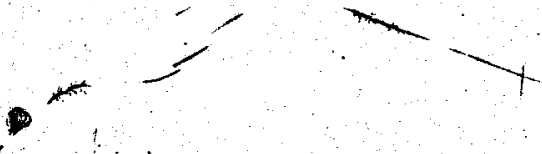

aecrio 10

CODE Memearun CAN 20 .

WECL VURCAN 2

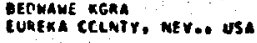

sanpt inc ineormarica

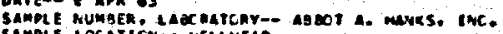

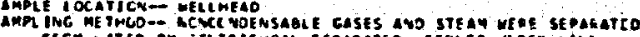

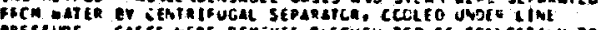

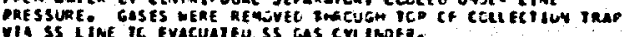

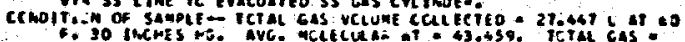

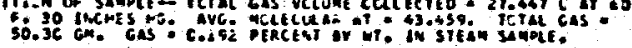

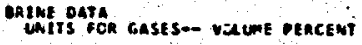

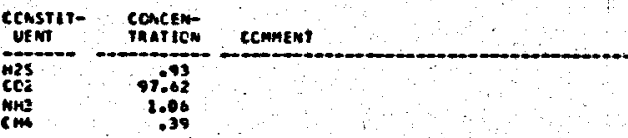

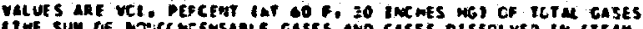

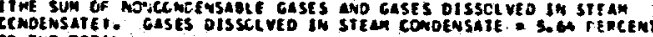

etoltegraphic cata

SOURCES- os

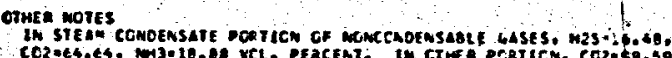

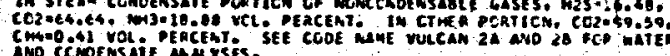

Meccho 12.

CCOE Mamerevecan an

Mete rurcan 3

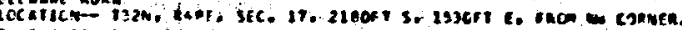

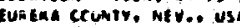

MELL INFOMATIOM

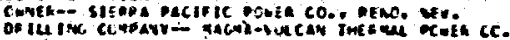

meteriat

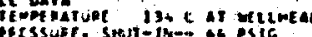

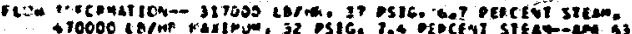

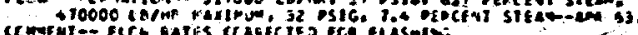

saneling emforsation

DAfi- 5 AOR Q

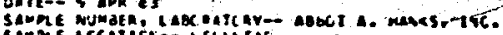

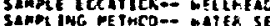

Monacerezusable

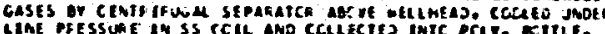

mrsicen oarn

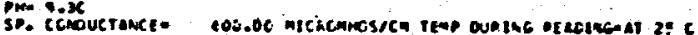

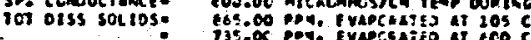

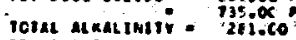

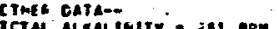

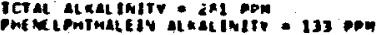

colme pant.

Censit- CCNCER CONET

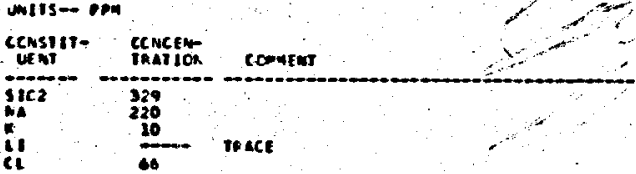

$\operatorname{sis}_{\substack{n \rightarrow 5 \\ \text { ind }}}$

$\operatorname{chs}_{\text {ict }}^{n+3}$

(c) 17

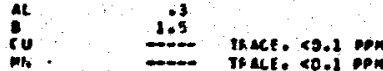

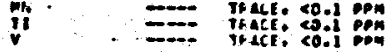

- iblioganpmic oATA

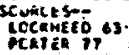

CTMEP MOTES

SEE CODE MANE - VULCAN 36 AMD 36 SCA CONJESSATE ANO

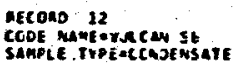

melt wuzan:

ILTES CCuNir. MEV.o usa

Sampa ine jafoination

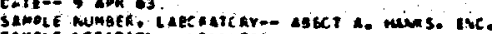

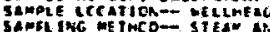

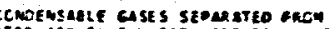

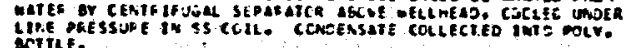

onstes.pata

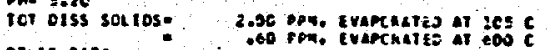

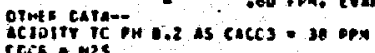

erime onta

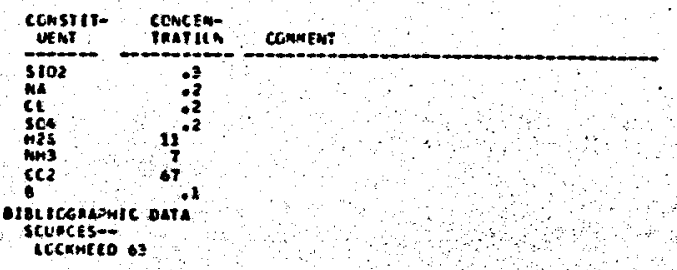

CTHER ROYES
SMELYSES. 
aeceas 12

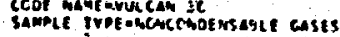

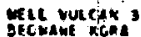

CuntKa teginiv. Mevo. usa

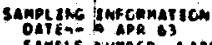

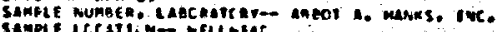

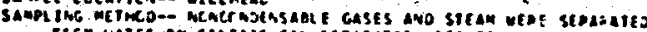

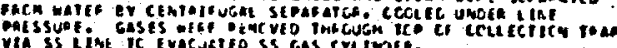

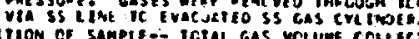

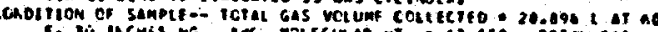

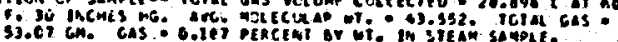

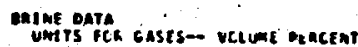

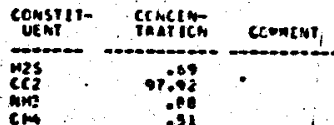

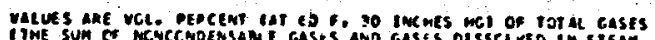

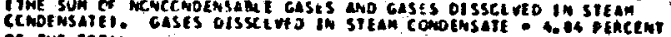
CF IME 10TA.

eselictarpmic deta

cockentio is

OTMER wOres

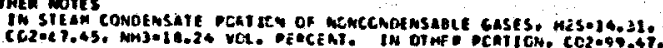

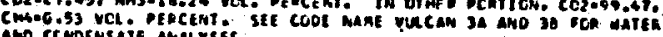

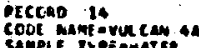

MEL vuLean.

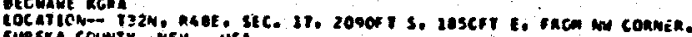

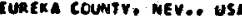

vell information

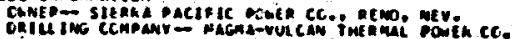

MELi OATA

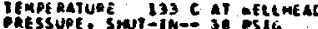

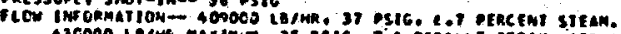

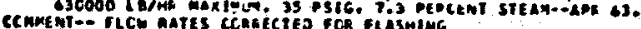

sanpline jurosenatice

gall- II Apa b3

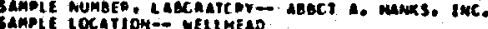

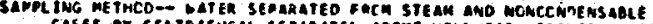

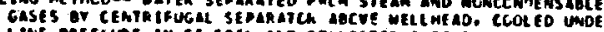

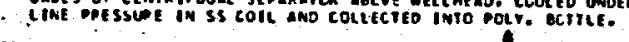

nirrsteal oata

St.: ¿ionouctance.

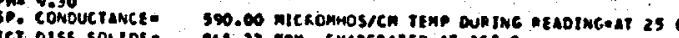

Itr olss soc los:

TOTAL ALKaL INITY - 7263.00

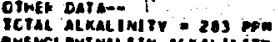

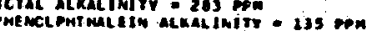

cerime oata:

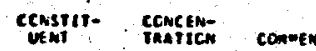

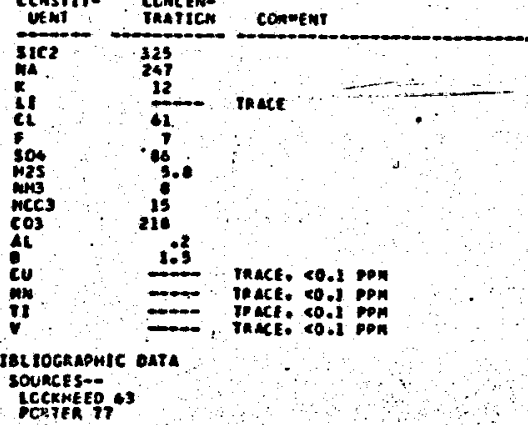

ctuex motes

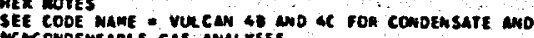

Mere nue tent:

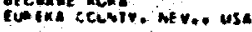

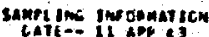

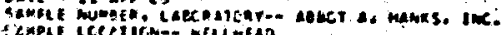

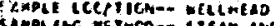

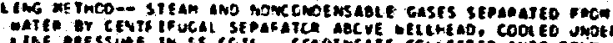

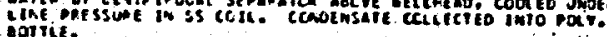

onrsical oente

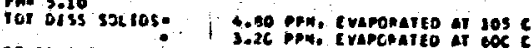

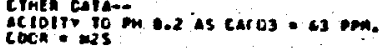

arine oars.

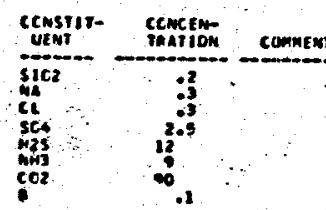

olge locenamic date

ITCKME

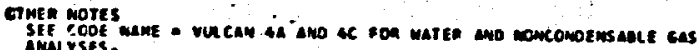
aecono le

vite vulcan

tionexa coumir, nev... usa

sanpling two gomation

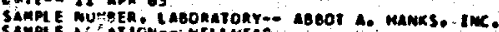

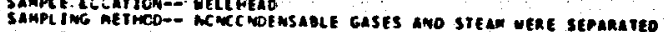

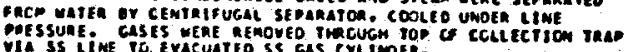

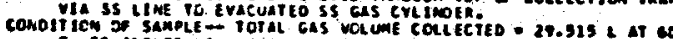

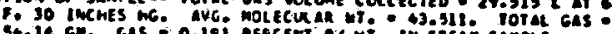

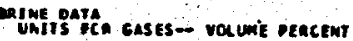

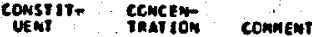

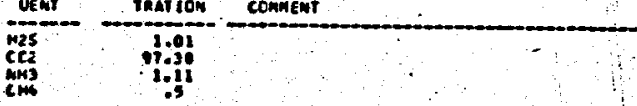

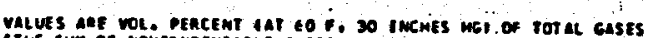

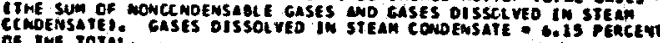
of ImE rotal.

elol locramic data

SOLRCES-

OTMER MOTES

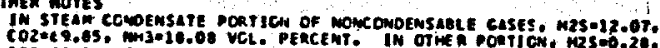

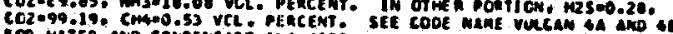




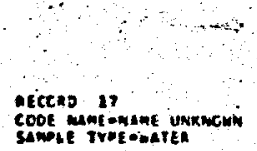

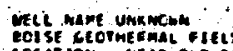

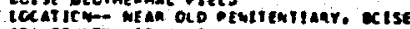

ade cCUNT, to., use

wect osin

Temeghatue De

ontsical onta

Tor oilss soliose $200.00 \mathrm{~mm}$

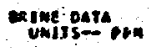

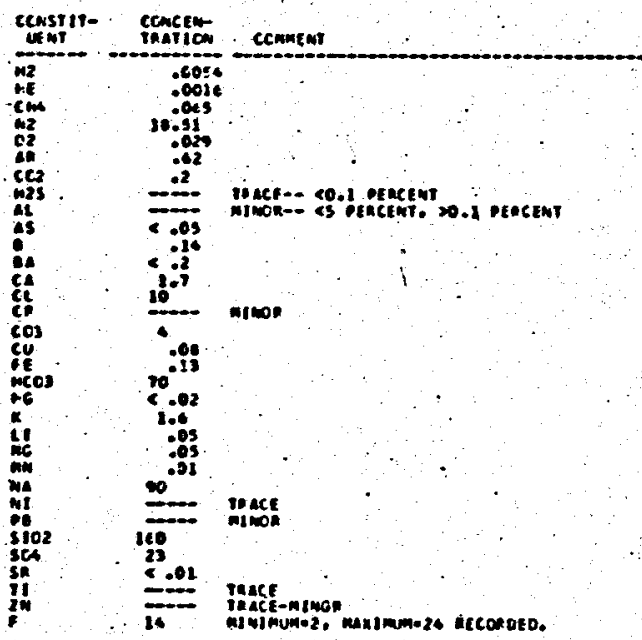

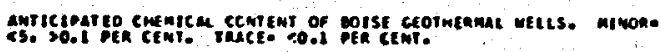
olot rogapmic olta

scuncesto.

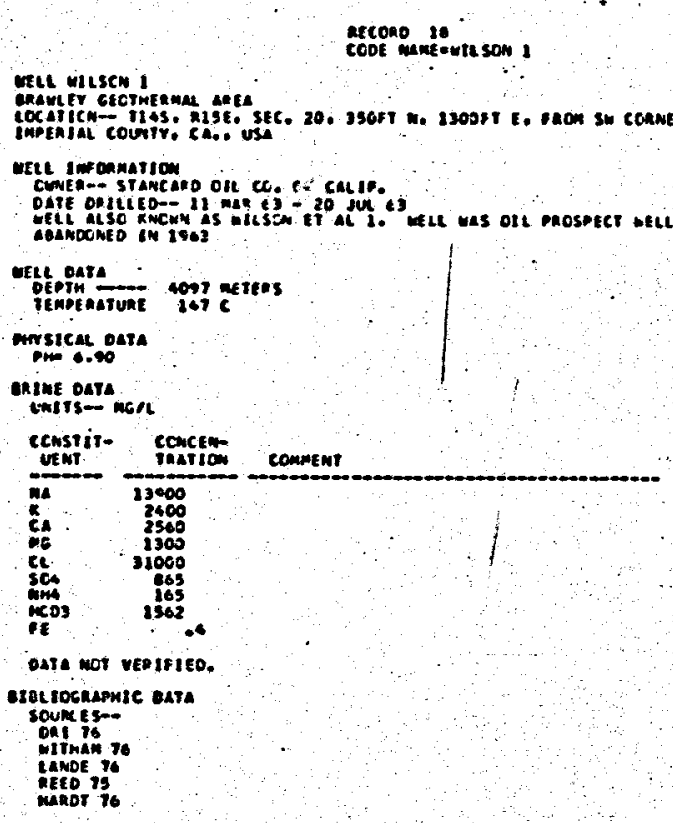

17

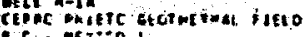
e.c.o mz:s"

SAMPLing inE ohmarion

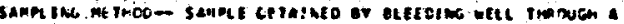

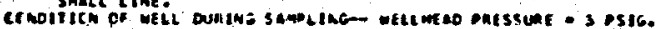

orine cata

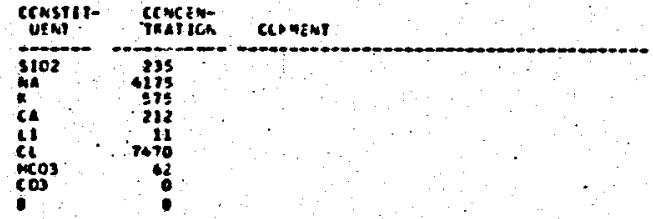

cieclocramic data

aracaoc is

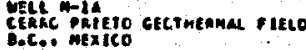

Sempl IMC IMr Denat Ite

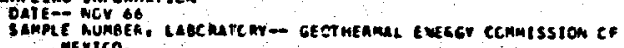

gime onta.

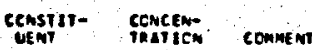

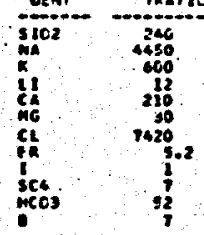

erellograpmic data

Souscts--

sopreving no 


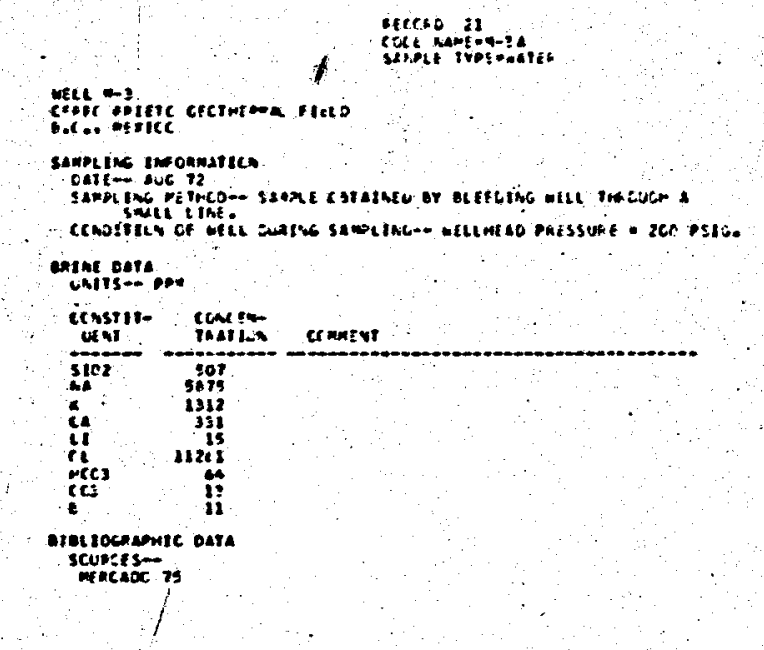

1

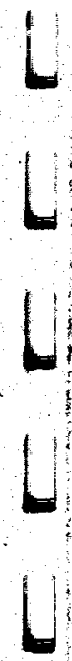

b

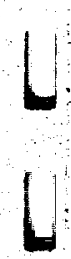

4
4
4
4
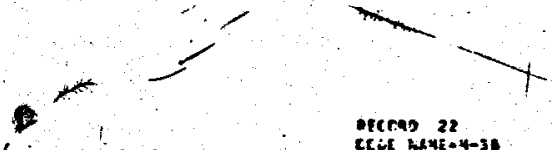

excras 22.

Sctite

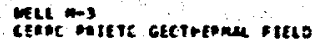

Bor.o misice

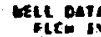

Senpl IAS Ims Jonatices

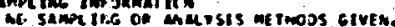

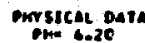

extuk oante.

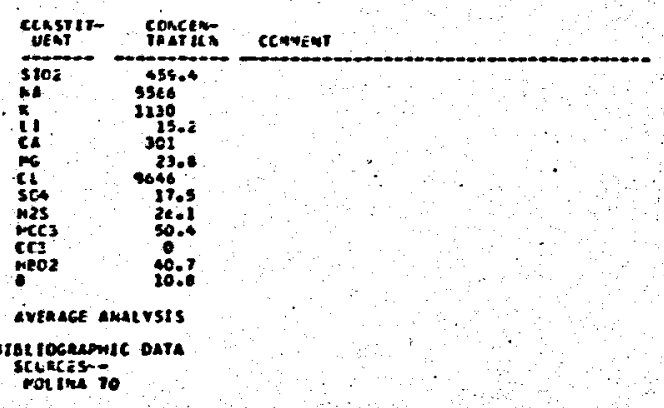

Recha 23 .

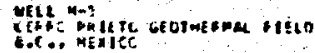

Sampi InE INFORMation

parsienc oals

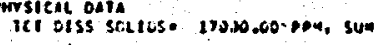

exint oute

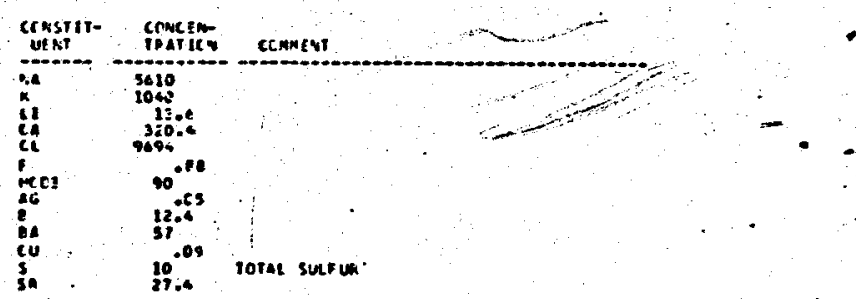

electiccraphic onta

scuacts-:-

cciun $77^{\circ 0}$

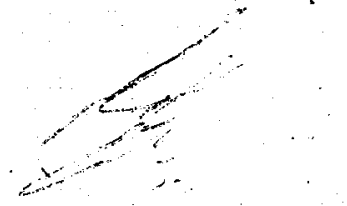

excens 24 is

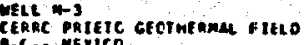

C.c.. MExico

SAMPL ING INE ORMation

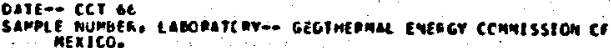

RRIME OATA

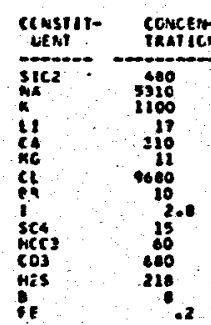

- tol hogenaphic oata

scurcesso-

conterio no 


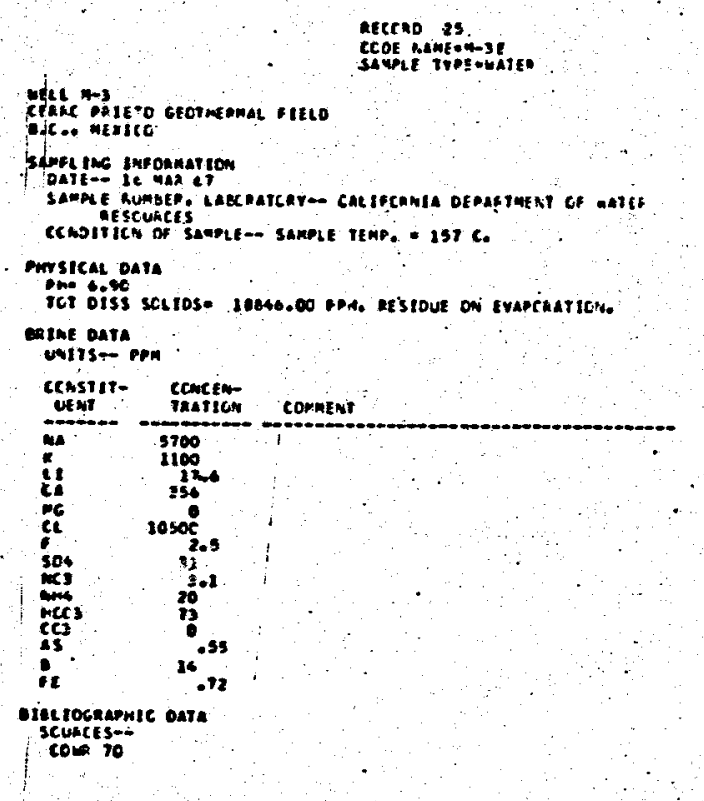

\section{GeCCAO 26 (G)}

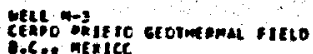

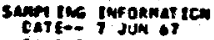

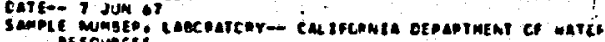

mine oave

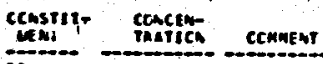

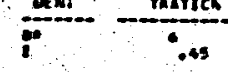

olgezograpicic oata

coxolic

\section{9}

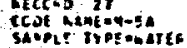

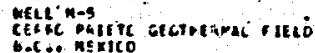

uthe imforanilon

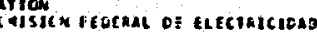

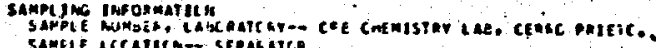

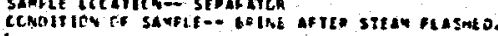

Prysical data

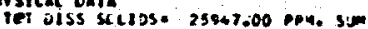

metne onta i

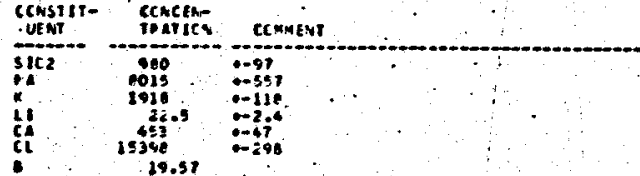

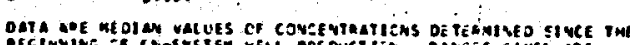

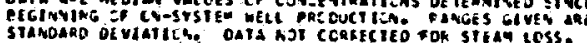

STAnoarg ot viatic

eloz cocanpme disia

$\operatorname{scunces} \rightarrow \pi$

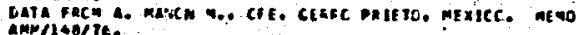

\section{Secono 20}

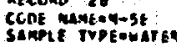

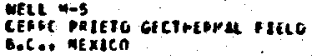

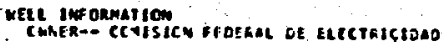

wele oata

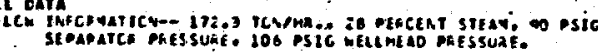

SAMPLIMG im Domatice.

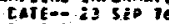

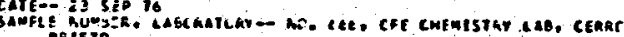
SARPLE RCGATICA.E SEPAFATER

BuIAE afTEn SIEAT Flasme D. Taxes, at

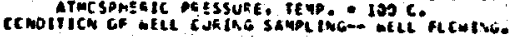

pursical Dath

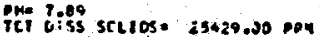

onine outa

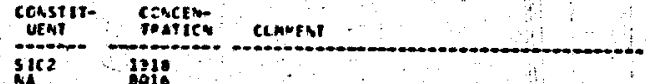

सा :

Ci $1 \cos ^{22}$

IL $140 z e^{\circ}$

in 23

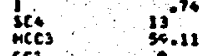

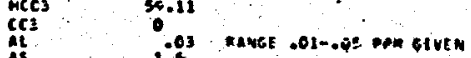

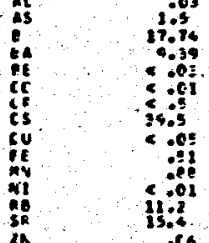

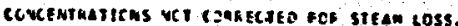

osercocrupmic oate

SCUECES

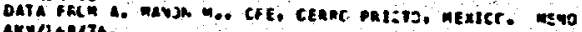





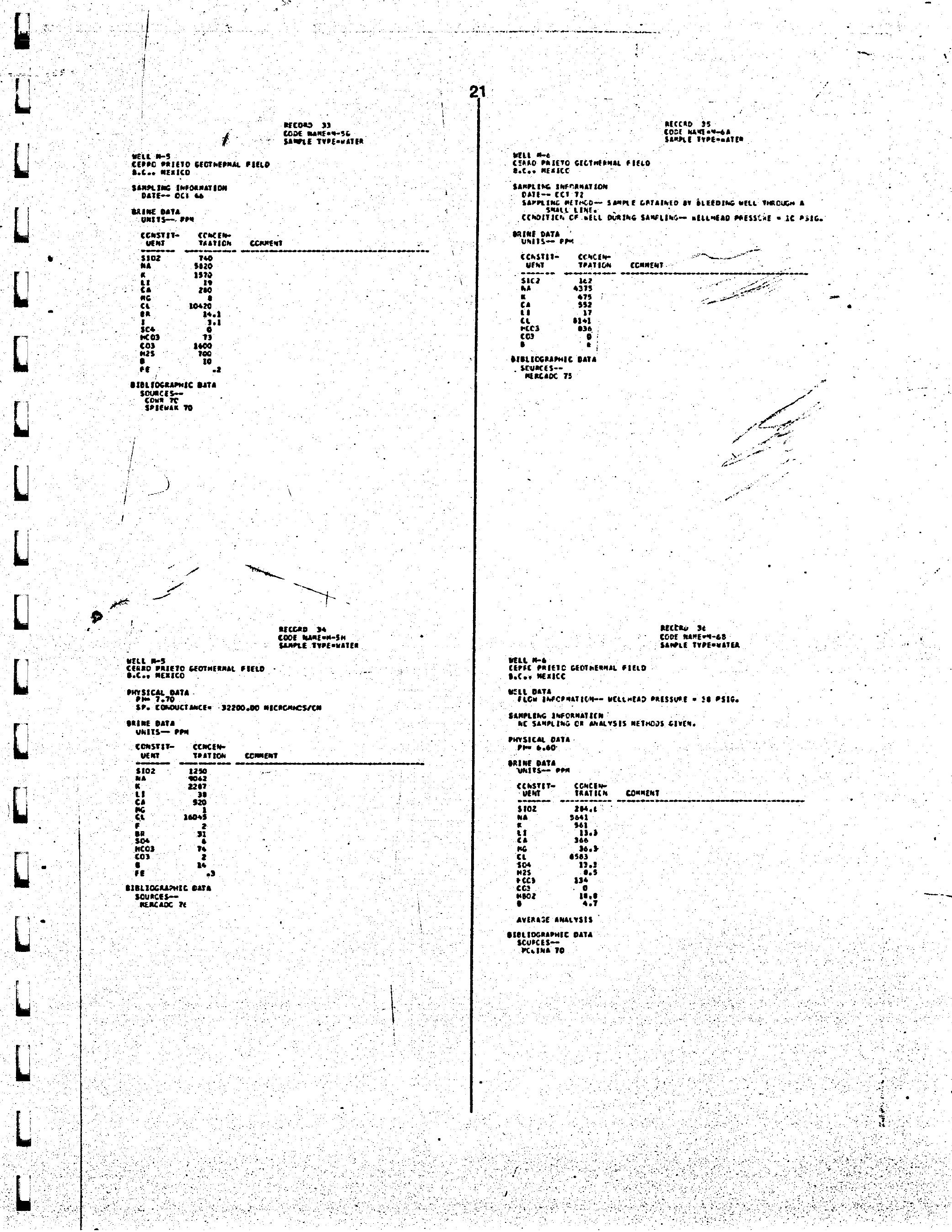




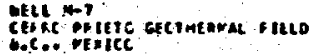

sanpe ing imformat ick

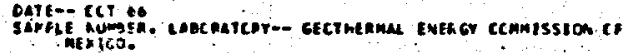

colme pare:

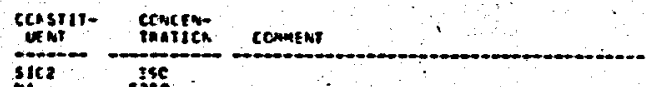

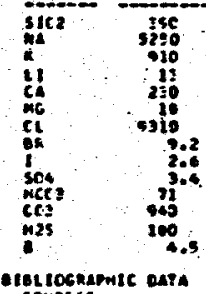

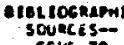

ccuk 10
settenex

\section{RECcro 42}

COOE MaUE-YY-AA

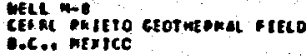

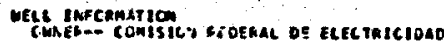

sampl jas imfonuit :en

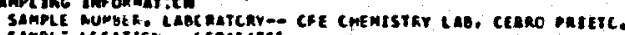

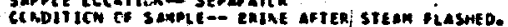

minsical Dals of

mine oata.

consyit- ccancen-

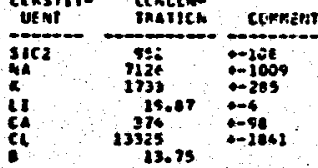

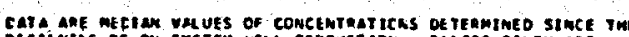

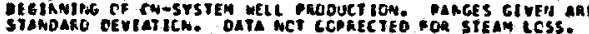

eral Hograpmic oase

Souncestio

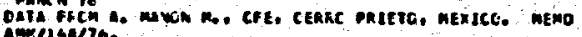

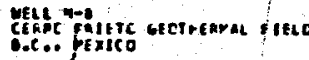

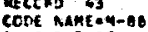
SLWh THPEonten

mene incemation

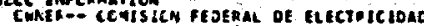

WEic oase

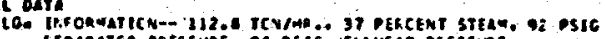

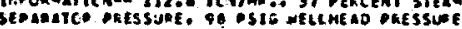

Sanptitic inf caralich

DAlE- is step stich SAPplé Eecaitica- sepafalca

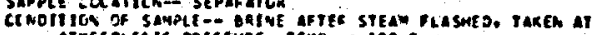

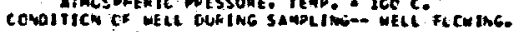

morsicm onts

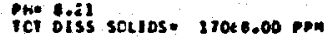

DRIME DATS

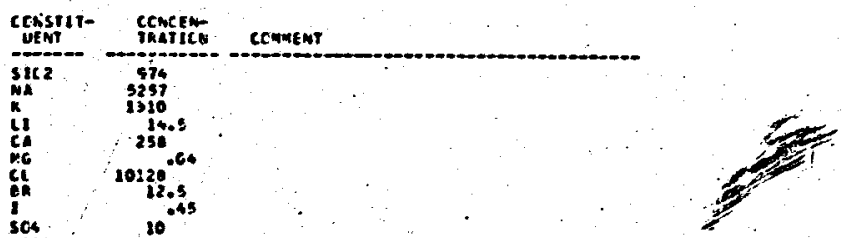

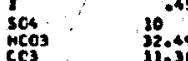

Cla

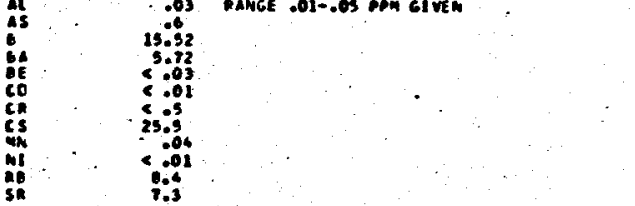

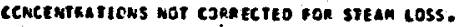

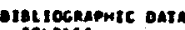

scincts:-

PANA FFCM 2. MANOW Mo. CFE. CERRD OKIETG. MEXICE. MEMO

\section{AECEad at}

CCLE MaNEAM-BC

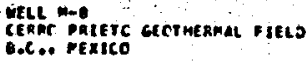

well oara

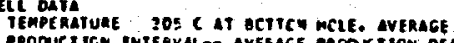

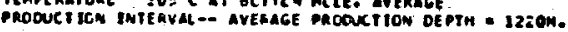

SAMPLIKG EMFOAMATIOA

onvsicat data

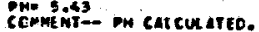

orine Data

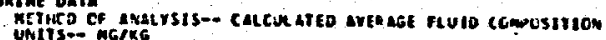

ccrsitit- cencen

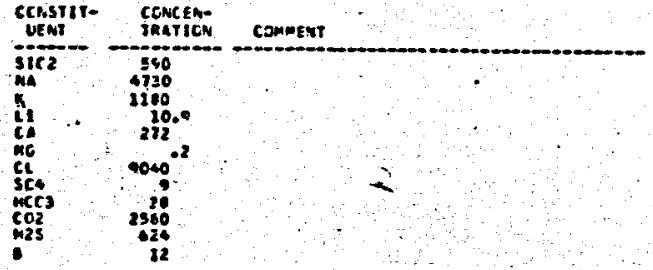

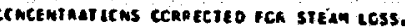

olbilograpmic data

SOURCES- 


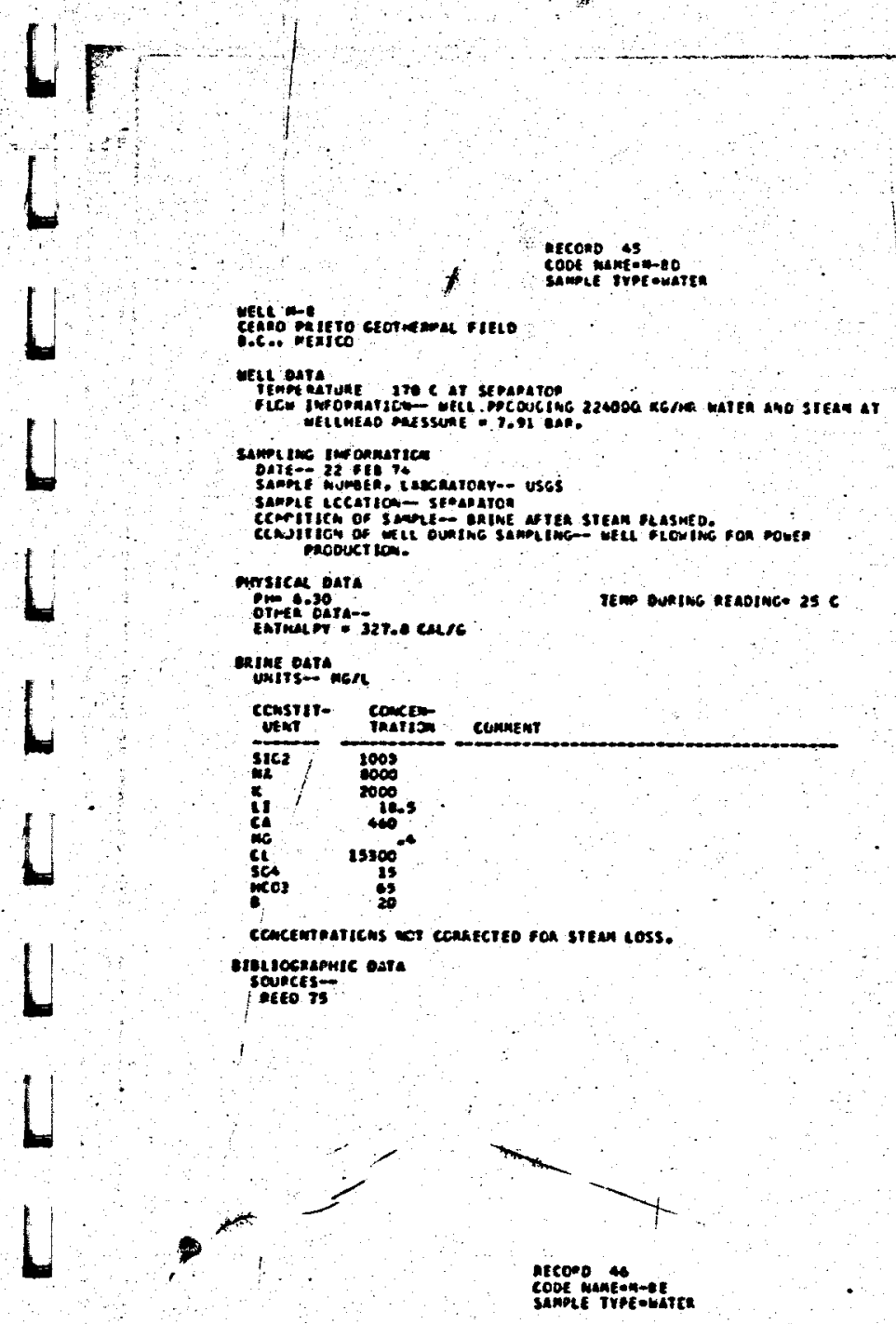

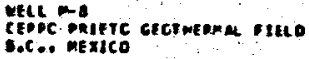

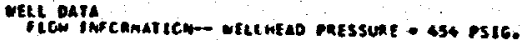

sanpling intoranaticm

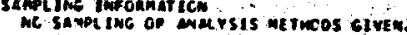

mistcat and

calus paras

cosstir- towefre coment

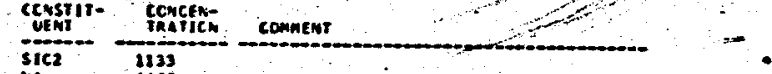

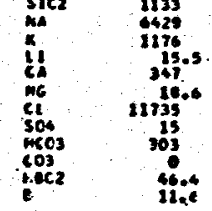

averuge amalysts

Bigciogramic oura

scencestina 70

afcoas 47.

SAMPL treserate

netiva ro

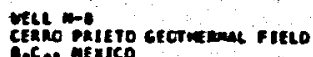

C.c.e mexico

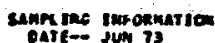

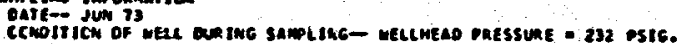
exime eata

unitss- pon

ccustito conces

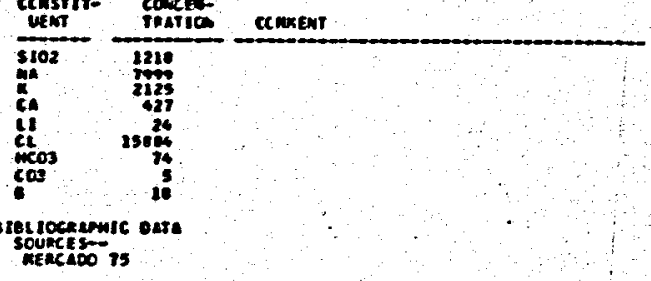

4
4
$y$
4
4

XEX $A D D$ is

\section{aecers a

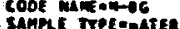

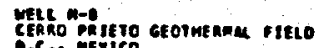

-.c.e mexico

Sampling IMFonmation

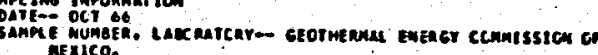

oxine paitu

Consy't CONCEN-

UENT iRATIOO CCMMENT

$302-710$

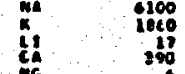

ct. 11730

Sct

- seg lockapmic onta

TSMR 70

glaxe it 


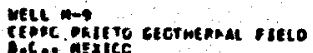

weut dita

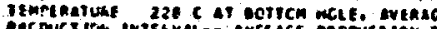

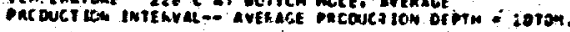

sanpline ine ongutson

SAMPIE Aumath. LARCEATCRY-- USES

onssicar para

ctrminto in calturerso.

enrme oure

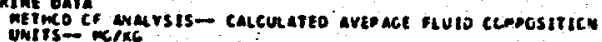

comstil- cencesto

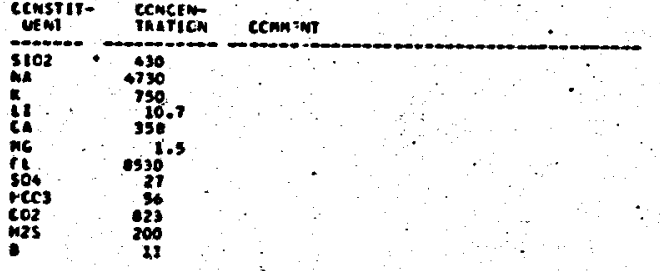

Cemetminations eckhectio ofer stean ICSS.

- migizeanamic onta

$$
\begin{gathered}
\text { sturets:- } \\
\text { ateo }
\end{gathered}
$$

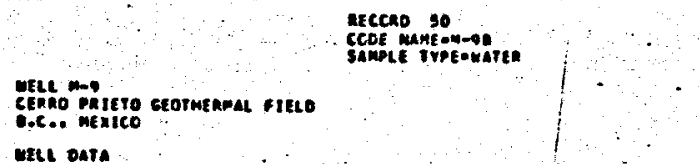

mete oara

Tempinatuse $200 \mathrm{C}$ at sebakatica

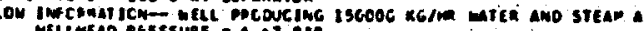

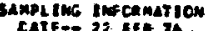

CATE- 22 2Et. Th

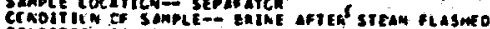

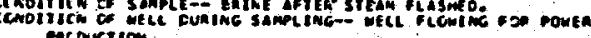
monocition.

nersicis onts

Timt:

chrmalov -.236 callo

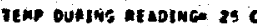

colme pars

Censit?- Ceneten-

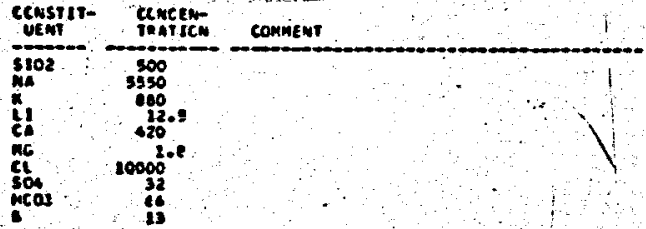

(3)

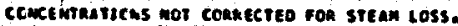

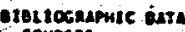
Soukets is

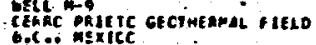

eegrat $=1-2$

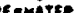

sanpling jaf oenarion

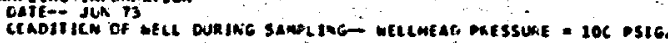
exime oats.

uniss $\rightarrow$ oo

- conssit- cracen

Jient tomation comatent

Sicz 003

Na.

CA

acc

cc3

sectoctuente outa

scyreess--

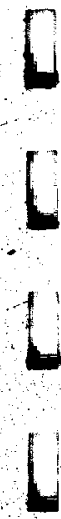

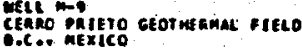

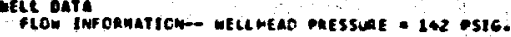
Sampl Ime tingoenasion

mystcat oath

cariue outa

CCWSIIt- CONCEN

Therism CJMNENT

3102
402
41

sovicess-: 
aecceso 32 CCDE MAME EH-10E

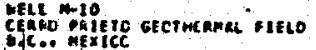

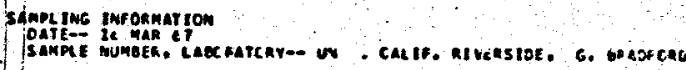

enine oara

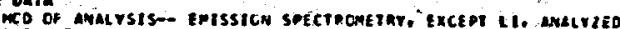

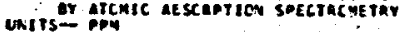

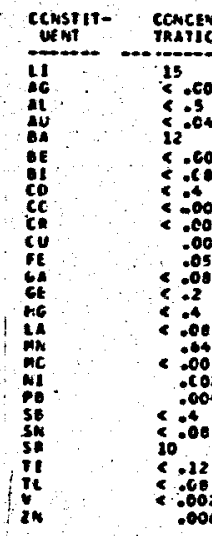

iss Hocrapuic Data

scyaces:-

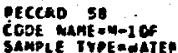

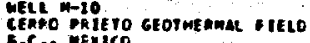
G.C.. MEnico

sampe the temf orenaticm

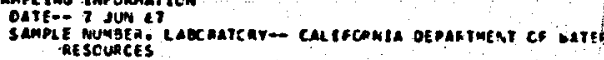

mine outa.

ctustit- concen-

UENT TRATICA COMMEAS

3

oleg cogarmic oata

Scuzces--
EEt m-12

i.c. ptnice

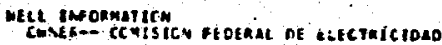

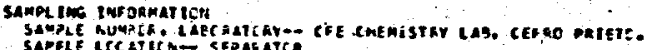

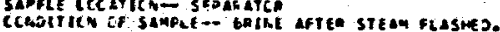

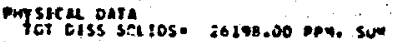

unine outs

Censin:- cencen-

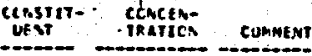

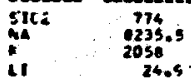

II 15.2237

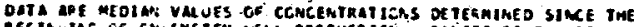

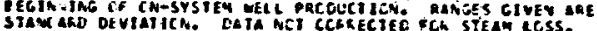

- iol tograpmic data

scasess

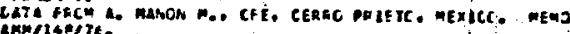

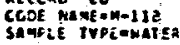

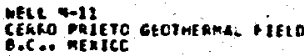

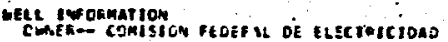

MELe outa

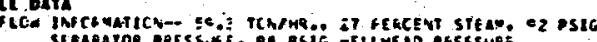

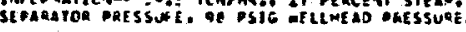

Sampl Ins imf odmaticm

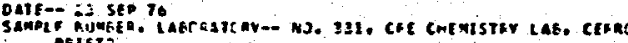

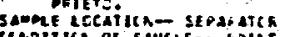

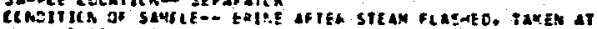

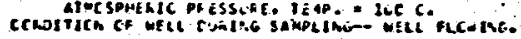

emrstcal date

Ict isiss sculos. ins:3.00 pop

ening onis.

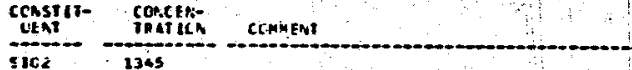

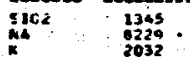

Cl $\quad 2032$

Ci $\quad 26139$

sto

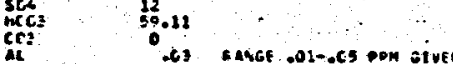

As $\quad 31.1$

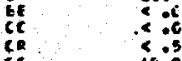

is

COMCESTEAT ICNS ACT CERAECTEO RER STEAM LCSS.

- jellocespmic oata

sences 76

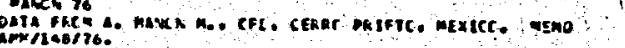




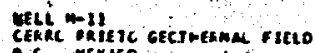
Cocor nexito

mele anta

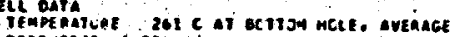

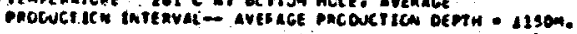

Samplatur impormatien

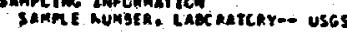

oursicm 0ata

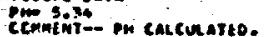

\section{eatre ousa}

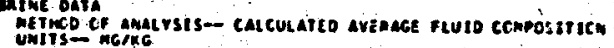

constrit- Concers

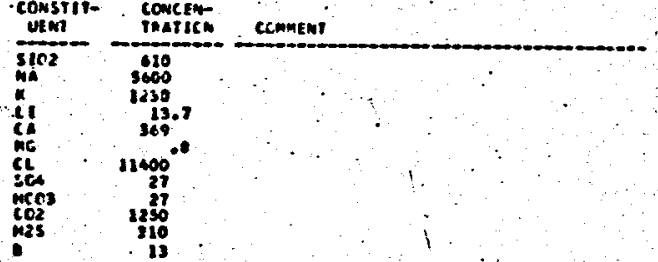

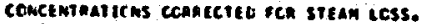

mezocsupmic oata

sourctes--

afCORio al

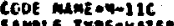$$
\text { Derco is }
$$

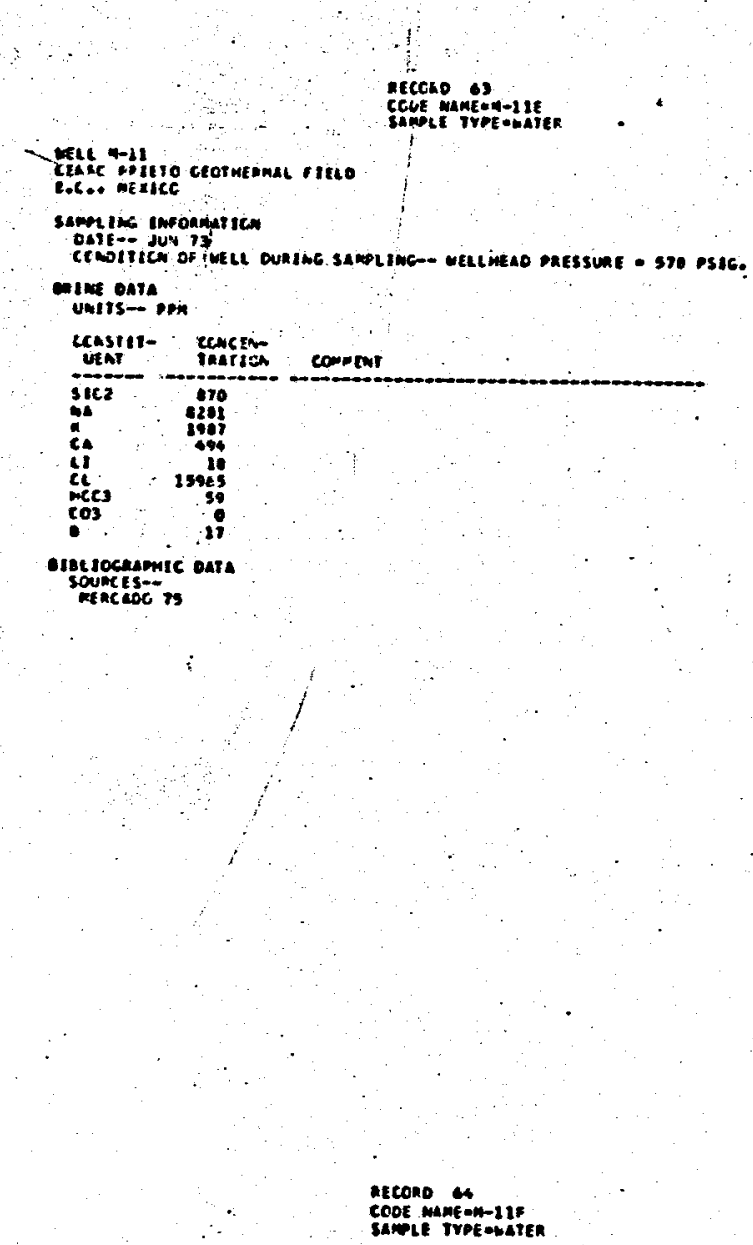

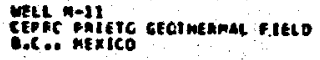

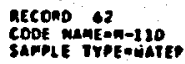

meteoth

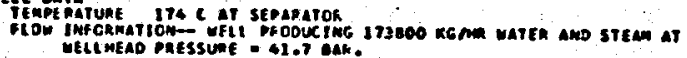

SAMP ING ImFonaticn

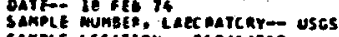

SAMPLE CLCATION-SEDAHATCA

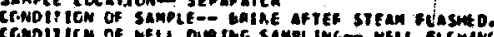

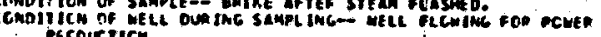

orrsicat para

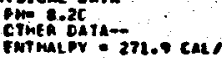

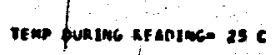

ourME PATS

ccustit- concero

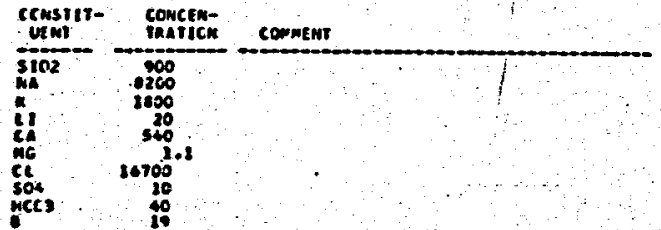

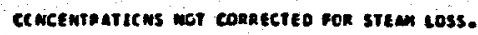

orizeonapitc outa

Stefe is

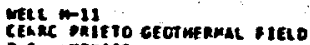

C.e.e. intice

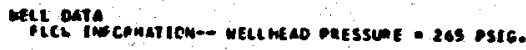

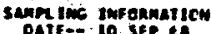

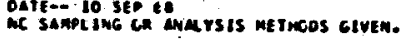

$\underset{\text { onsicu oarn }}{\operatorname{mox}}$

mime oata

unisso. om

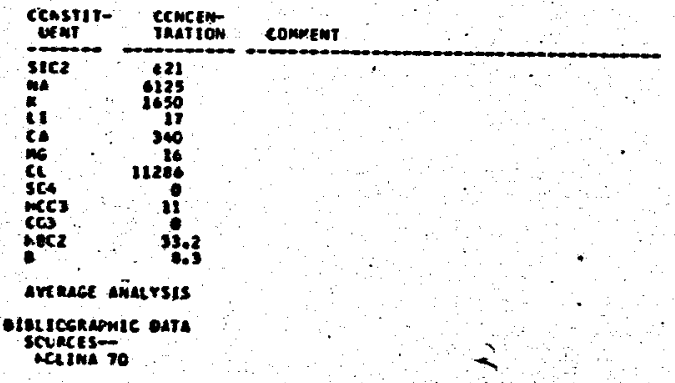



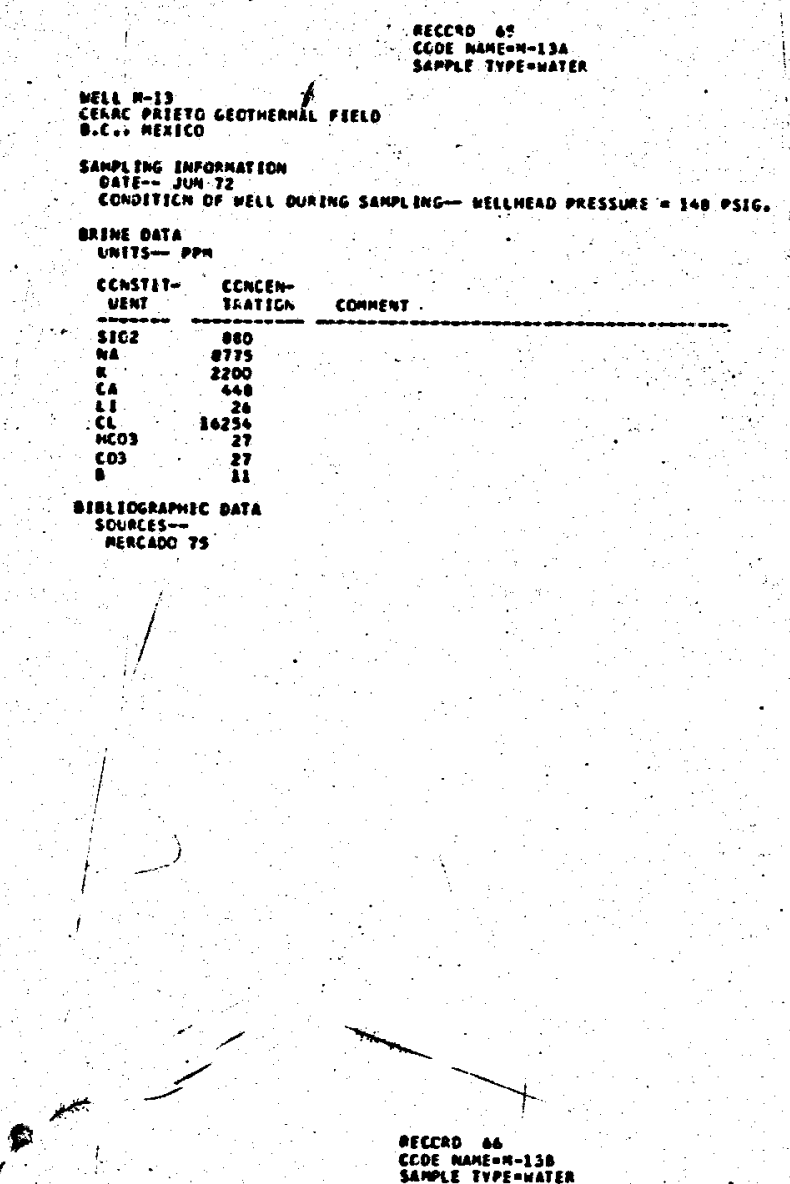

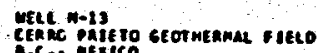

wetr oupe

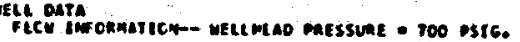

semplens infoswatich

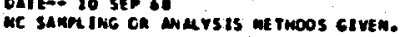

mrsical oara

enjue oata.

CCASTIY CrMCEN- CONEMT

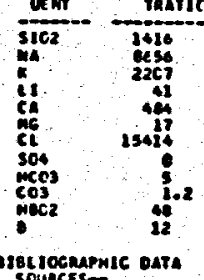

souncess to
1

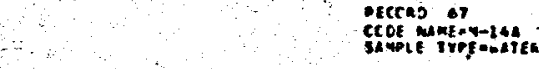

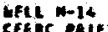

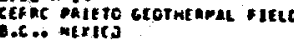

MeLl infCemation

tomeno- comiston fideral of Electaictoido

sampling infogmatisn

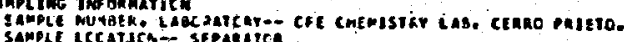

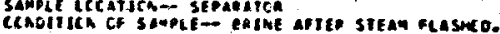

erystcat paja

ensme oase:

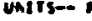

conswit- concen-

$\rightarrow$

cis 12710

Conment

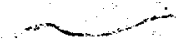

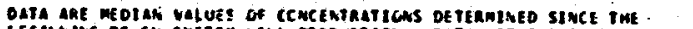

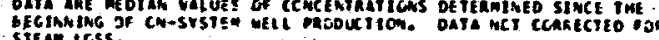
algirocrapousc oase

Sounctess-i-

cartionck at. (a)

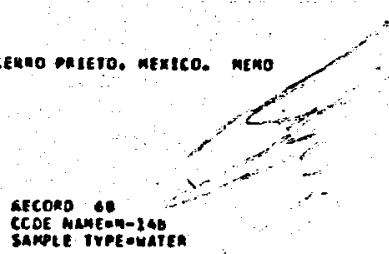

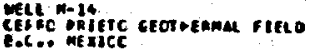

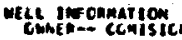

wELt Oars

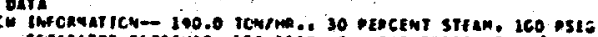
sampline information

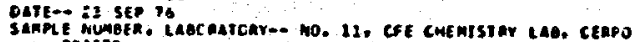

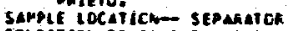

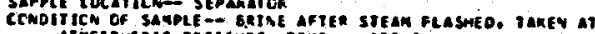

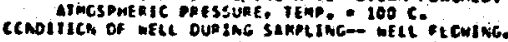

morsical oata

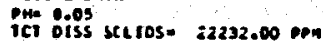

oxime oara.

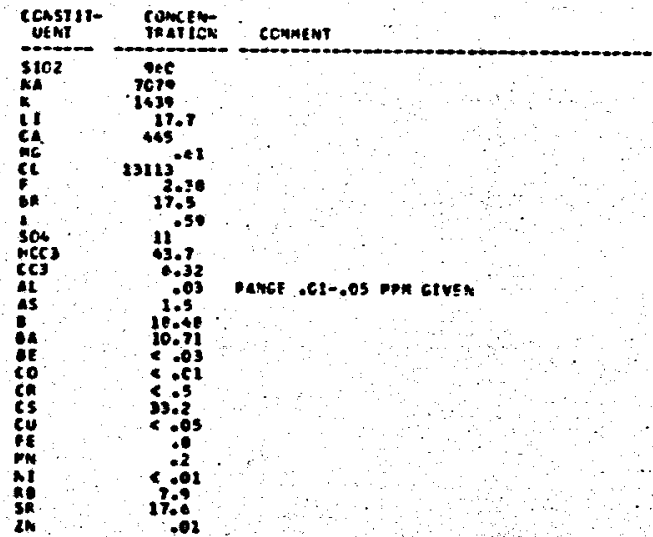

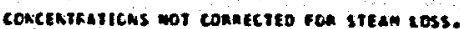

Btol HOGRapHIC oATA

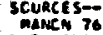

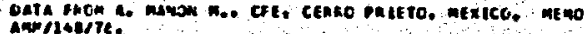



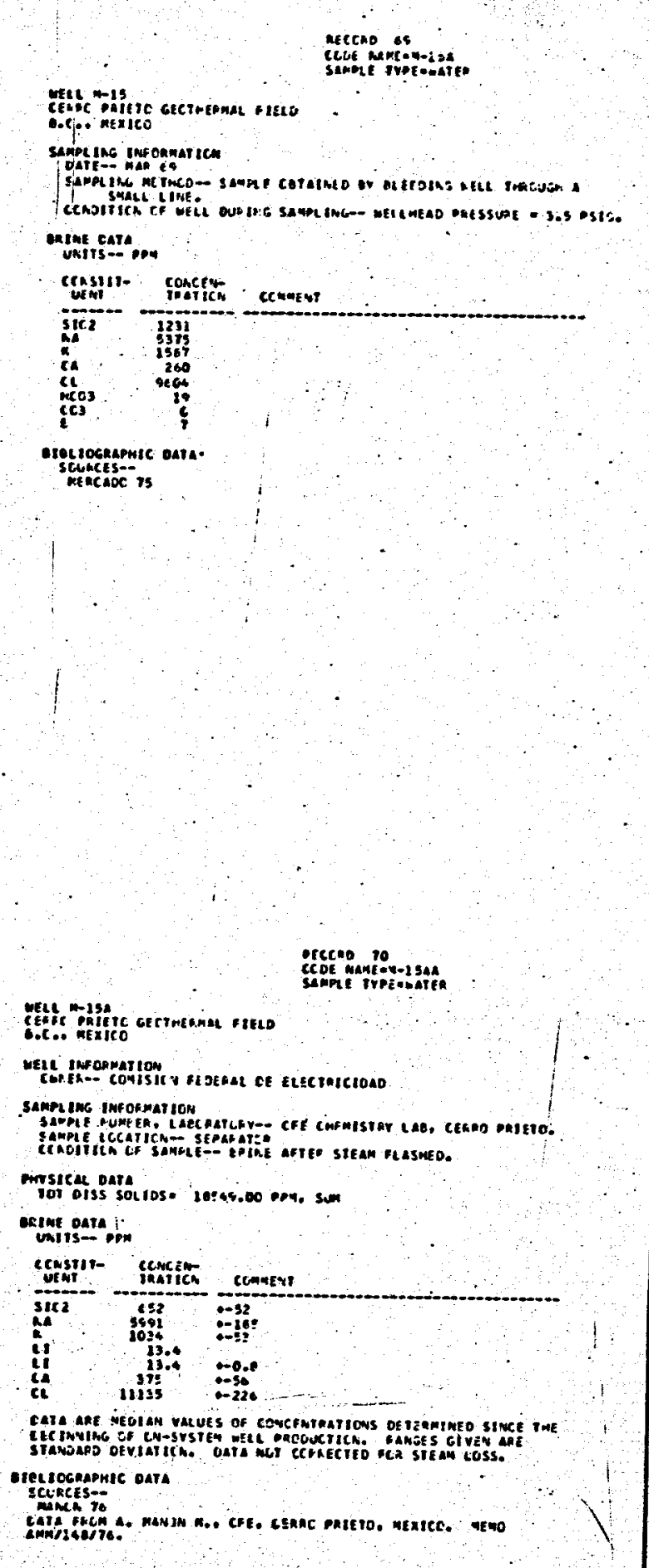

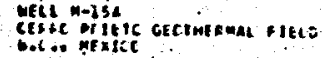

ctelt exscamaition

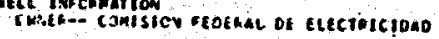

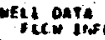

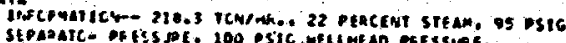

sanpe ens insogmanter

Dale $\rightarrow$ is is iffe

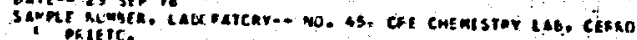

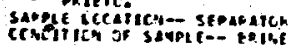

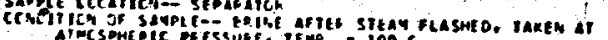

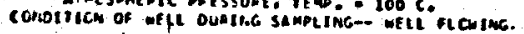

onvsicali oaja

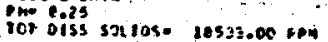

encine oure.ion

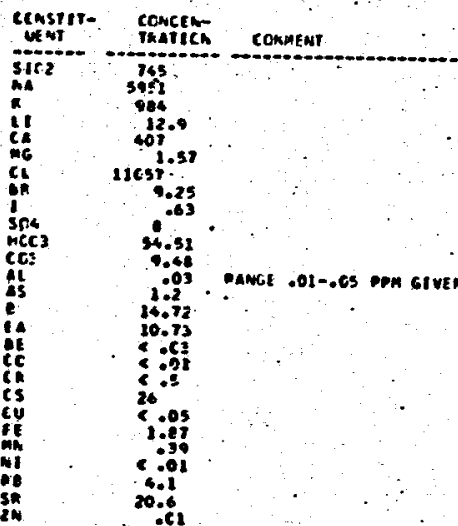

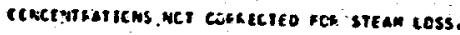

- geguegeapmic oata

nate 78

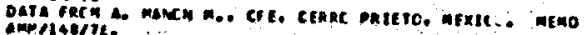
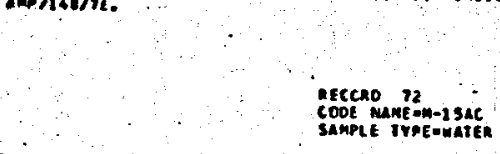

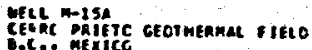

sanplyac informaicon

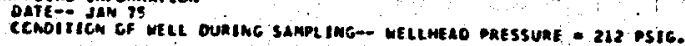

okime doaya

Censint - CCHCEN-

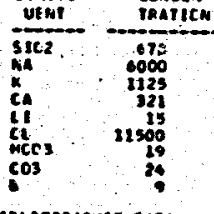

Comant

esoluograputc oata

Scuretsistis 
actord 73

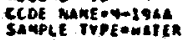

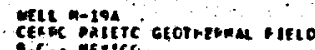

sc.. we nice

chl incormation

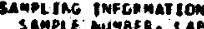

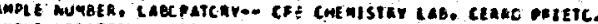

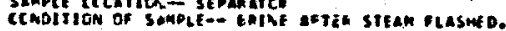

miticat DATS SOLLLS = a7497.00 Pry. Sum

unike outh

censtil- cencein-

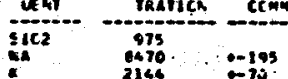

CI $\quad 234.54-70$

ce 372

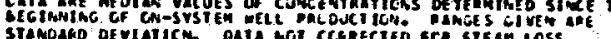

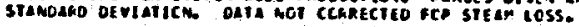

elel sographic onts

seuscest:

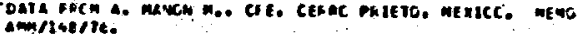
amiserre.

\section{ieccios 74}

\section{CODE MANE ${ }^{4-16 A B}$}

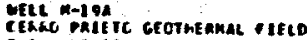

o.e. mexice

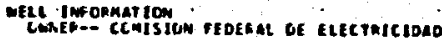

meze OAYA.

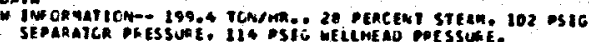

Sampl Ime imformation.

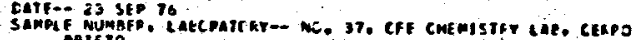

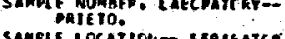

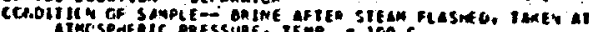

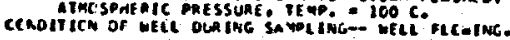

misical OATA

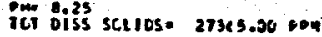

eane oara

consilt- cencert-

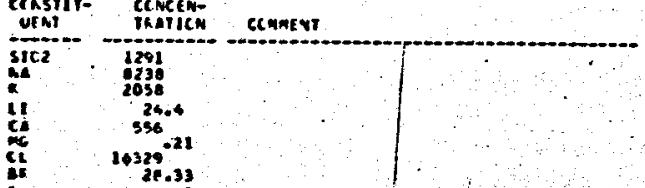

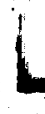
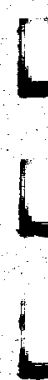

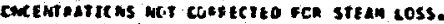

grezicgrapmic oate

scukcest-

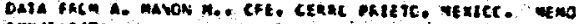
asmollatise.

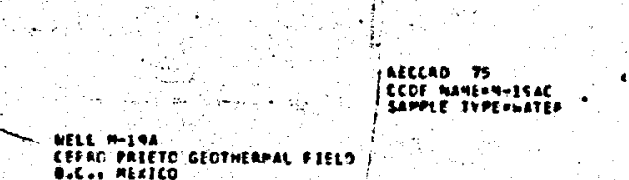

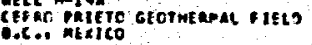

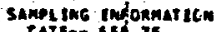

tation III 75

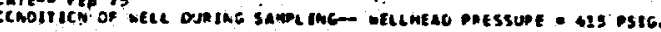

anine oura

vints - -09

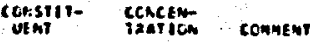

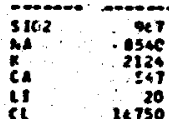

- tectocrepaic data

SEURCES-O

atccos 76

Ccok nintoy-ios

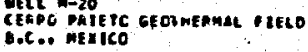

weth oara

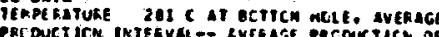

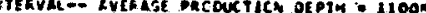

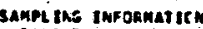

SADPLE aymaER. LAPCoATCar-- uses

onrsical dara

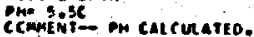

ERIME DATA

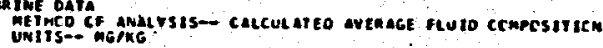

veting

CeNeEa

CCKEE KT

312
41

(1) $\quad 10$

sed

sed

${ }_{\text {H2S }}^{25}$

Ceacemiraticas ccerectlo ion stean euss.

eleztogacantc onte

stupcess-: 
accono o1 CCUE mantoy-2HA

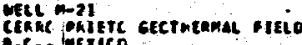

sanpling imponnaticm

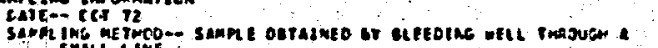

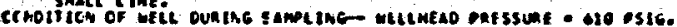

Exine oATA

ccristit:- canceri-

Utmi CCNCEN-

$3 i c 2$
ca
if
ncos
cos

its.

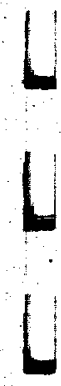

oret jockapwic eata

SERCAOC 19

Aeceno as

CCOE ManEm-218

Gete n-zi

Cocer mexice

wete anse

sampe tenc informat to

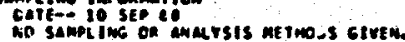

insical gata

cuime oata

Units- oon

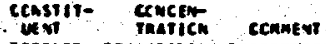

(isi

-

ccrimevi

is 290

(4)

et.

$\underset{\substack{\operatorname{sic} \\ \operatorname{ccc}_{3}}}{\cos 3}$

pece

34
240
245
48
3

crolsocaimic anta

scuarestion io

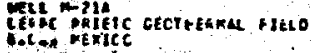

negt thenomaticen

sempling emeornatice

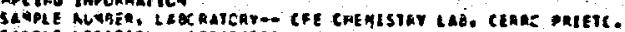

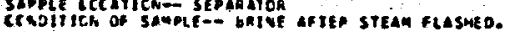

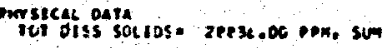

corve outs.

conspit- cencen-

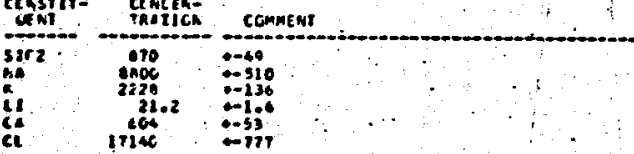

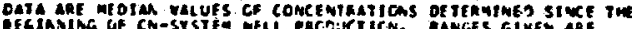

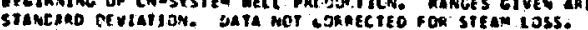

eseciocespric 0ate

secels

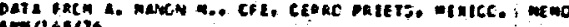

\section{atcono of}

COOE Maming-21A

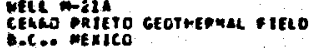

EEL jefcrmaylon

were oart

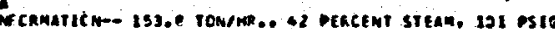

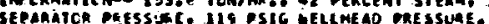

SAMPI IMG IMFORMATICN

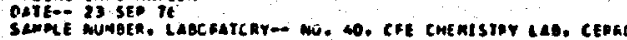
SAPPIE IOCAIICN - SE PARATOE

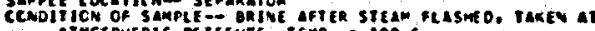

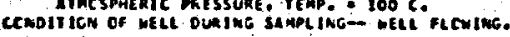

omesical data

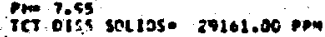

oxine oasn.

CCAsti1- Cences-

t104

in 2.25

CL 1727

CL $\quad 17277^{\circ 1}$

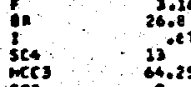

$\mathrm{CC3}_{0}^{\mathrm{mec}}$

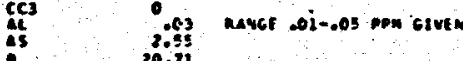

$4 \quad 20.7$

ce $\quad 80.03$

ca 18

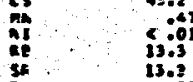

2k

orgiloskaphic Oata

scuects $-:-$

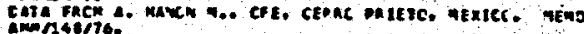




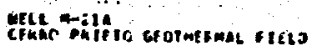

bocos matce

sanpline insuamation

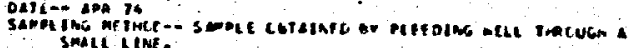

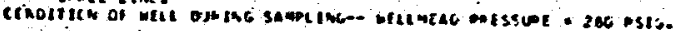
antme care.

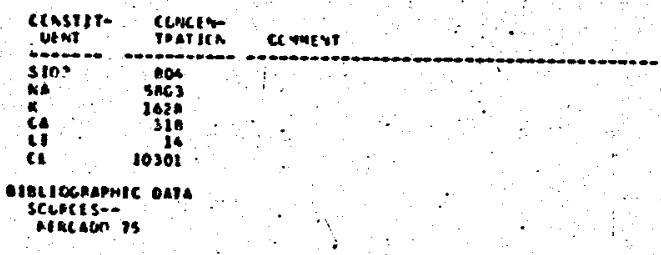

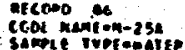

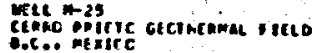

MELL ING ORMATIOH

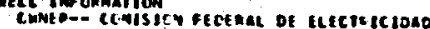

Samplane zompenation

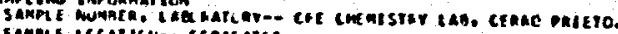

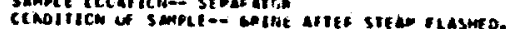

mersical onta :

ICI DISS 302 105. 36049.03 c004, sum

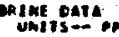

Censuir- concens

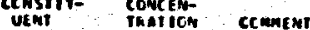

a.

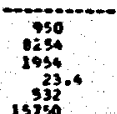

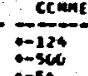

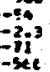

15.03

1

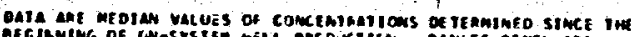

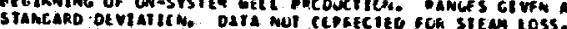

ergezocapmic data

Scuaces:-:

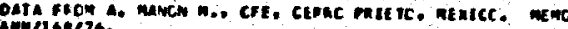

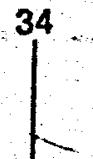

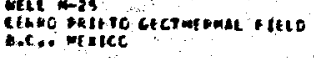

mate thochatice

witeda!

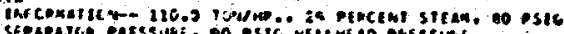

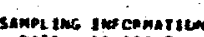

polfo is sfo t

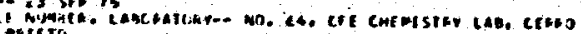

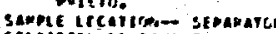

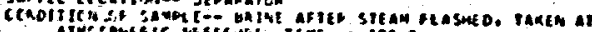

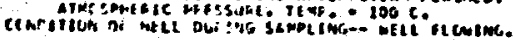

errsicat ceta

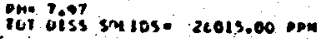

ourut oila.

censtit- concen

:

CEMmeni

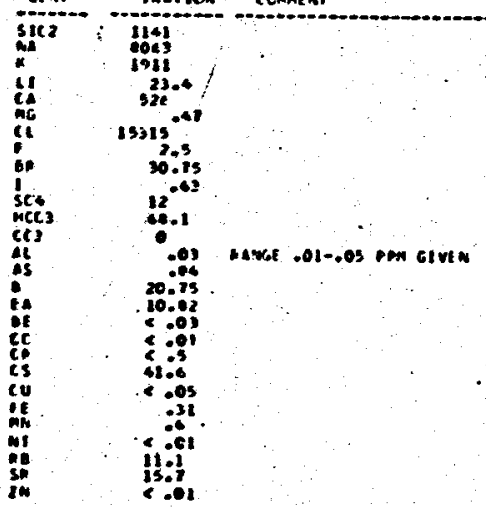

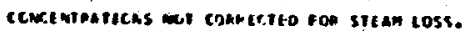

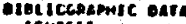

Sruces $-x_{6}$

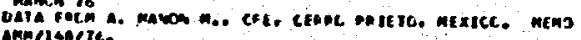

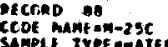

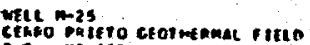

C.c.e nexico

wret odia

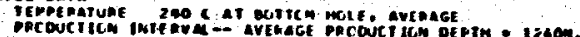

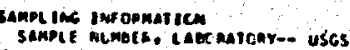

cane bata.

arito co anatrsis-- calculateo avehace huso compesition

consiot-

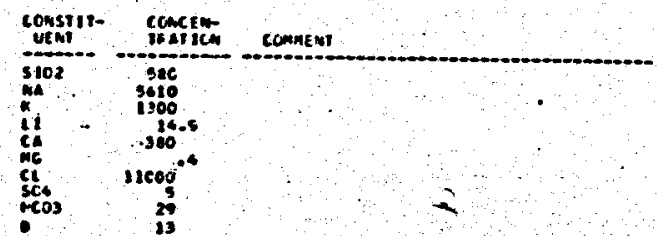

Cementanalens cconecteo oca stean coss.

isl gognaphic onte

SOUACE $5-$ 


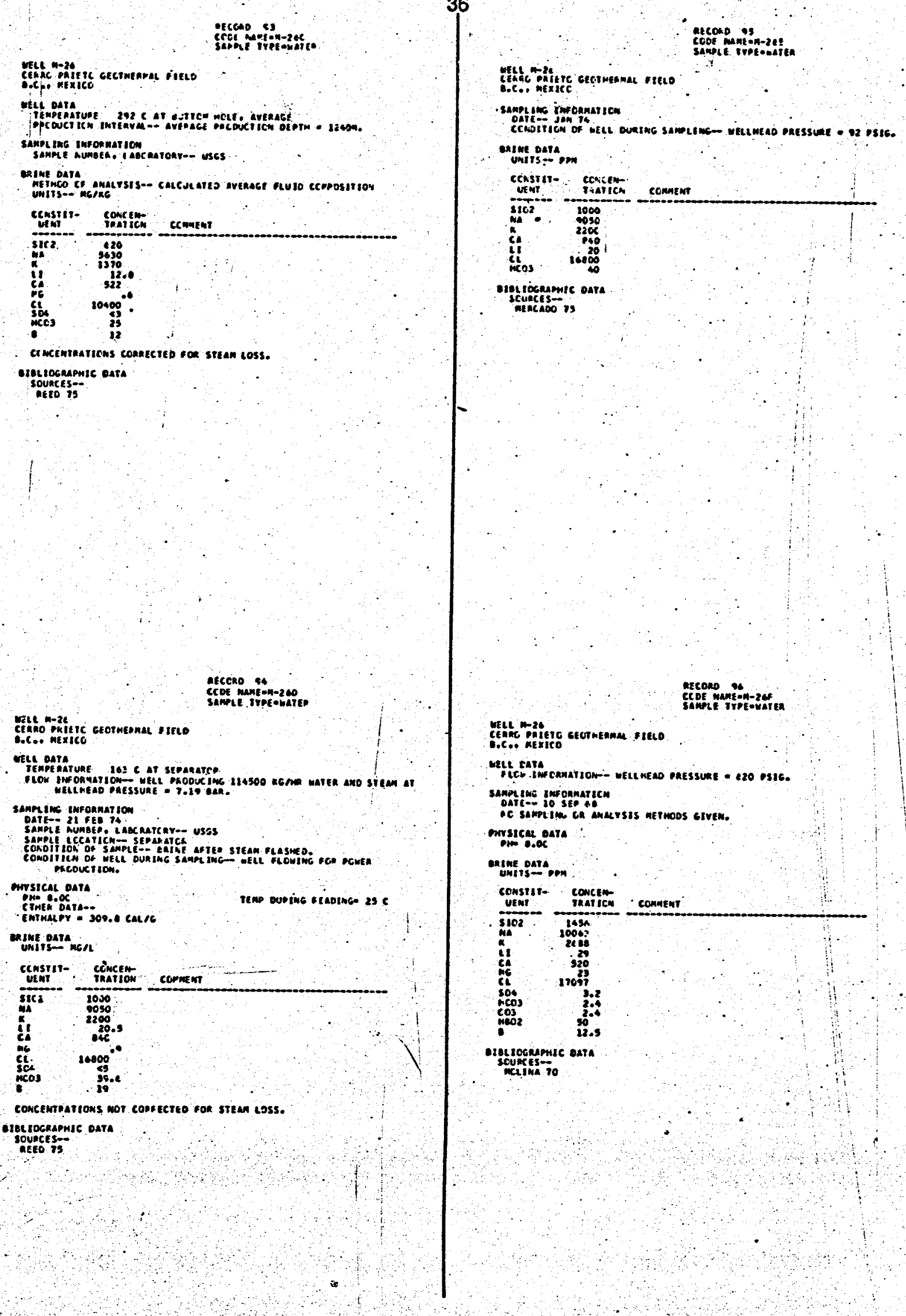




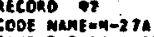

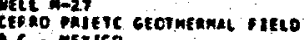

C.c.i mexies

ueit imfoamatjon

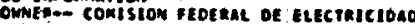

samperic infociarice

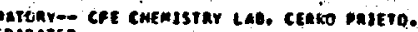

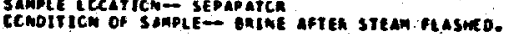

purstce oate

carme jata

Units- om

conssit- CCricew

went imeticn cennent

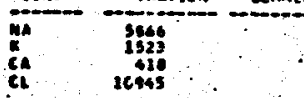

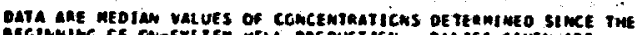

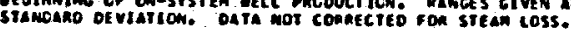

itez lockapajc oata

SOuces

DATA EACM A. MAMON M.. CFE. CERRO PRIETO. REXIEC. MEHO

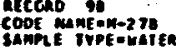

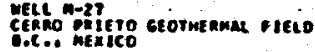

mege ireornayion

weLc odra

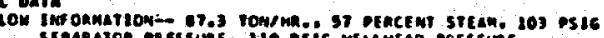

Sanqu ine juFonmar scm

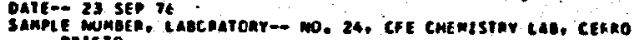
Sample LOCAYION- SEDanatca

comotilion of sample-- gaint after stean bitasico, taxen at

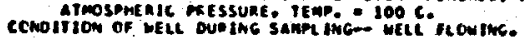

mrstcal oata

Tor ofiss solios = t9:53.00 opm

exjuk oara

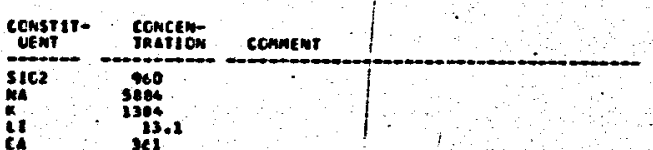

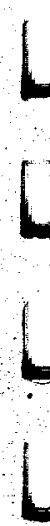

Rance .01-.05 con siven

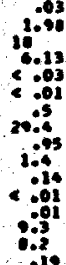

conetwruariows wer conkecieo ron stean ioss.

crot rockeme outs

Sounces 5

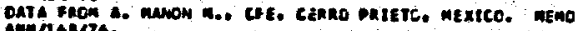

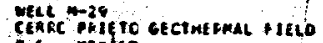

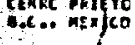

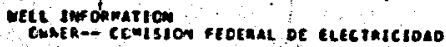

sumpting tmfonmatlom

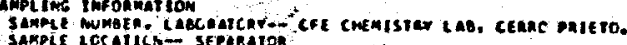

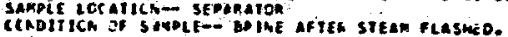

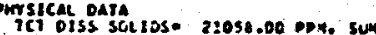

cutwe odia.

cchstit- CCAcen-

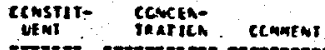

$5102 \cdots$

if 1120.5

Cl 12553

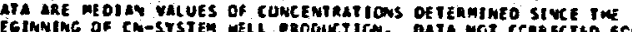
sientess.

eresicgrapmic oata

SCuRCES--

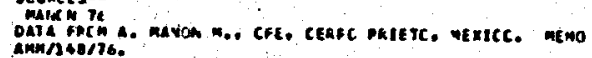

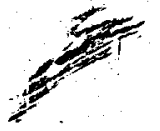

Aeccino 100

COOE NLMLOH-2SB
SAMPLE THEE

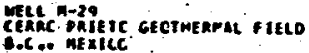

metingonalion

wece anta

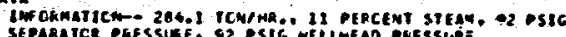

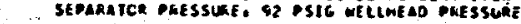

SAMPT ING TMFOAMATION

SATHE 23 SEP TE

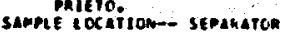

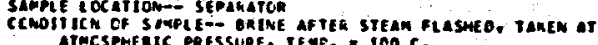

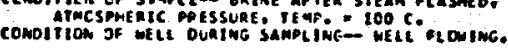

mersical onta

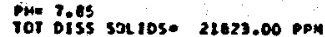

arime oata.

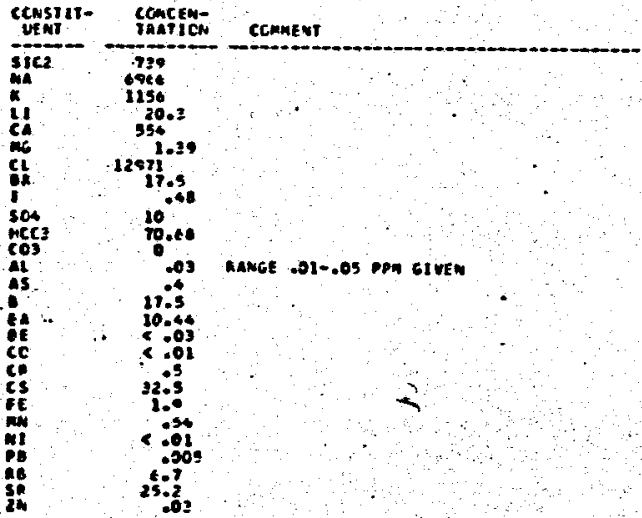

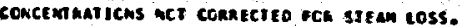

molgocmanitc odita

Sounces--

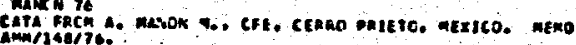




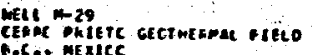
Goc.. MEXICe

mele oart

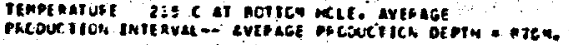

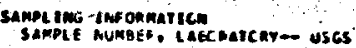

morstem oats

ecrivinte. pu calcunated.

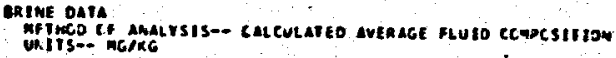

CenstrT- CCrCEN- CCMnEVT

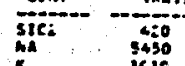

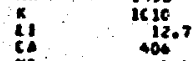

CL. $\quad 102000^{401}$

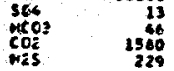

CCNCENTAAIICNS CCEREtrio ECR SHEAN LESS.

Cleziccapapictic data

SCustes is
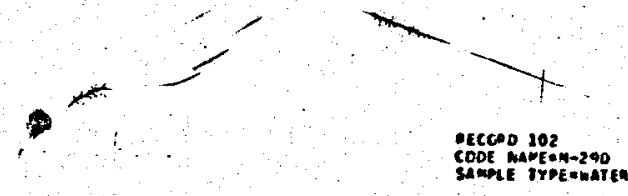

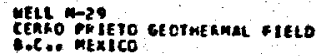

meti ana

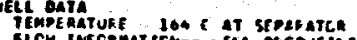

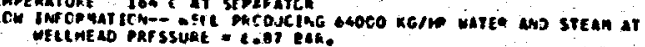

SAMPLing imformation

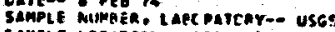

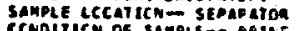

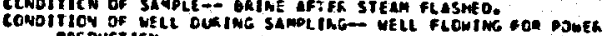

mrsicai ours

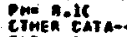

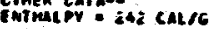

TEn ouning henolncias as

exime pata

CCNSIIT- CCNCEN

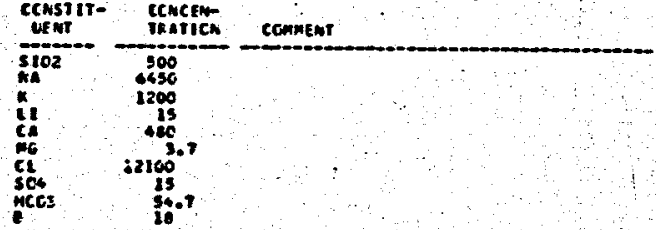

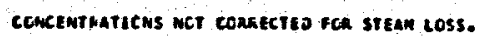

etelicceaphic oata

Sovect5:-

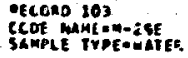

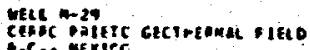

saxpiting ine ormation

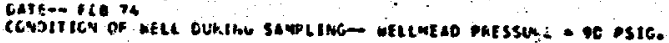

onime oata

ewiss-o pon

ccassul- cencente

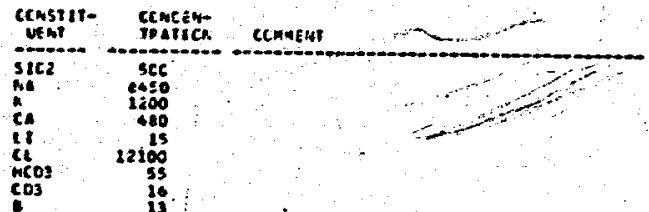

elge joganputc oata

scuketes- is

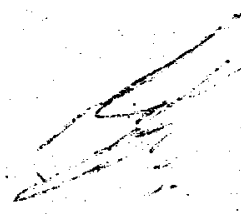

Recexo 204

CCOE MAYE-4-29F

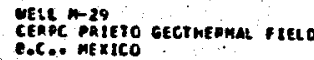

well onts

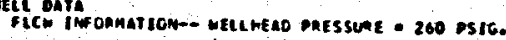

Sampeing Jurormation

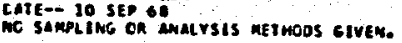

onvsical outa

onine oare.

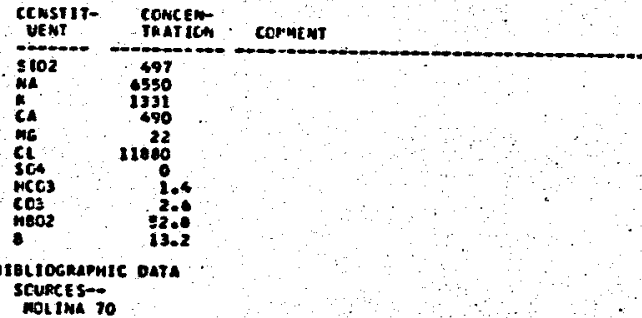


ACCORD ICS

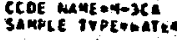

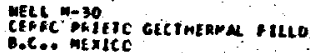

Decto mexica the ofmation

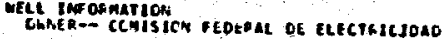

sanpe inci emronnaston

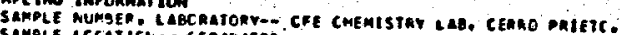

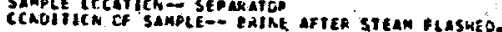

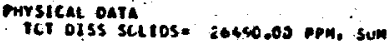

inzime oate

units - - opd

CCNSIIT- CONCFN- CLNA

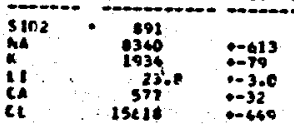

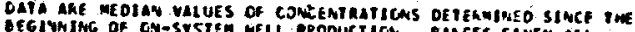

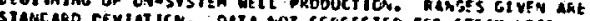

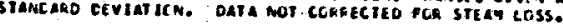

itsi jograpmic date

scureces

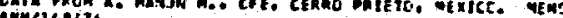

\section{AEcted Tle}

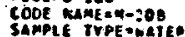

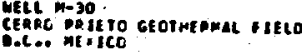

Hele infonmation

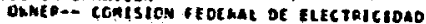

mete oara

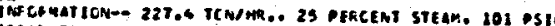

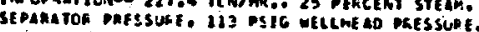

Sampl IMG Ims ormatices

DATE- 23 SEP 16

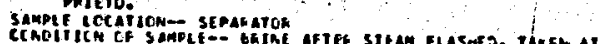

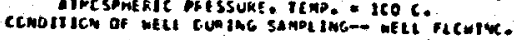

entrsicar cara

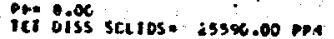

onjue oate.

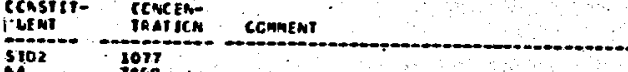

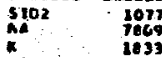

LI 3 ta $^{2}$

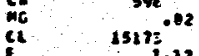

in 33.32

$\mathrm{SCCO}_{\mathrm{CH}}^{23^{48}}$

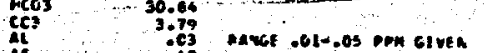

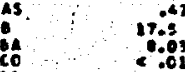

Co

is $\quad 38$

N1 $\quad 2 \%$

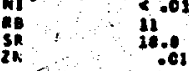

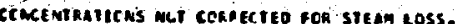

- gelgogapuat oata

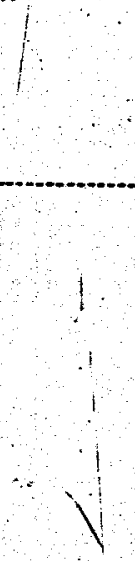


atcero ice

CCOE MAME-n-30E

veil m-30 CERo orizto geothearal fíclo

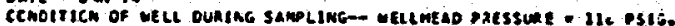
eaine onta:
units $-=-0$

CCNSTIt- CONCEN CON

HCc3 30

Scurces:-

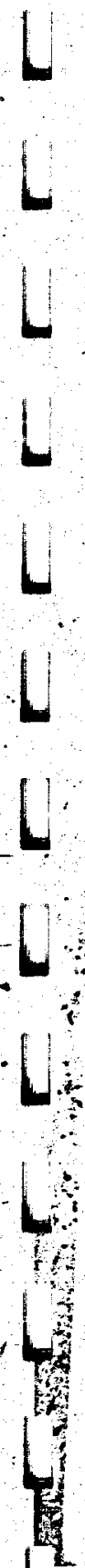

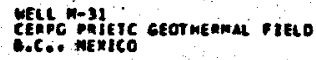

UELL EMFORMATICH

sampling twFonmaxion

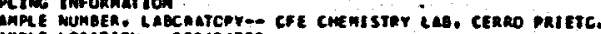

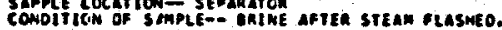

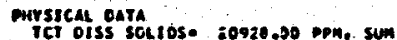

caine onta

Uhits o- on

GENI! CONCEN- Comment

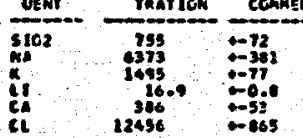

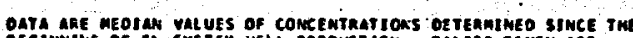

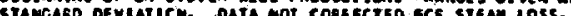

sellacaaphic oata

Sounces-

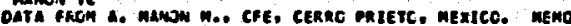
armaliserse. samplesc throngarion

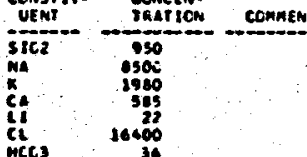

tccaio 111

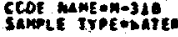

CEt: $n-31$

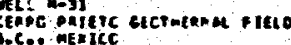

Mel information

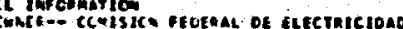

uets oura

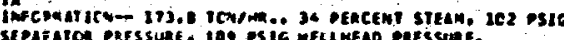
sepitato pressuat, loo osic uelakead pressune.

samplang iscomatrich

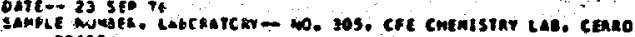
sampentery.

Sample eccalica - sepakates

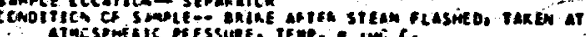

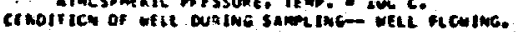

onsien osite

ivi biss sectios. 22264.00 opm

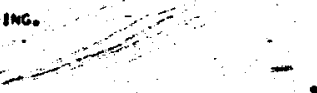

units oata:

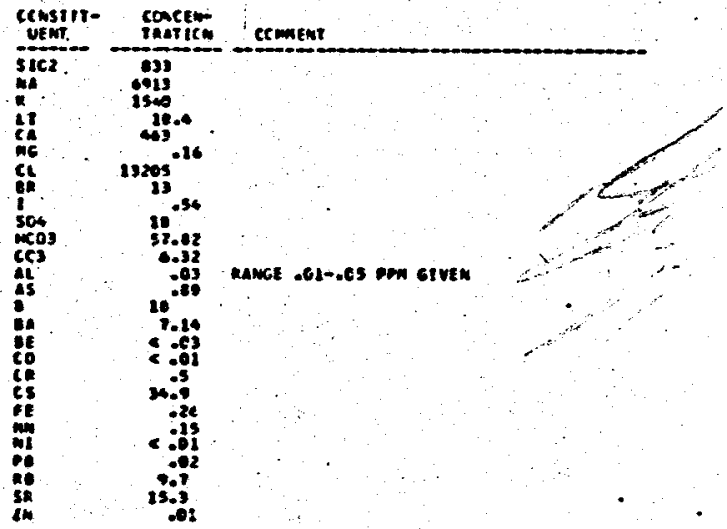

Cokcentraticins wot connecteo fon stean coss.

esectocanamtc dati

scurces 76

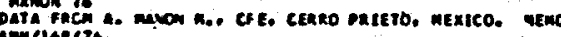




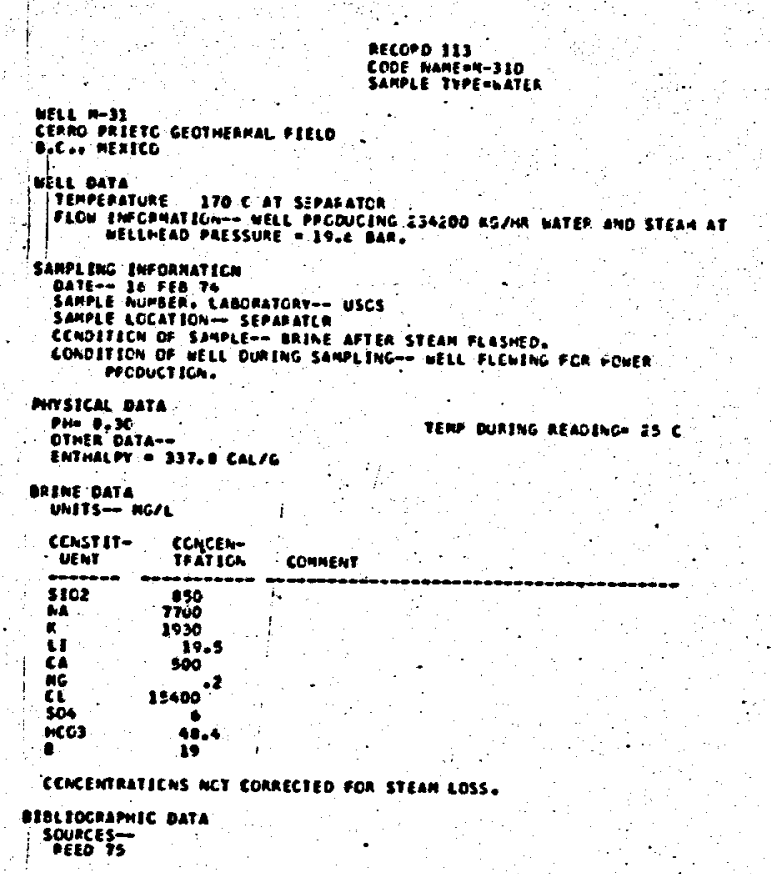

H
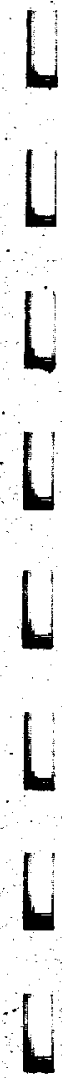

\section{Aecond 116} COOE MANEEH-3HE

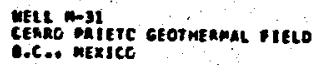

\section{saxpithe imfoaneiten}

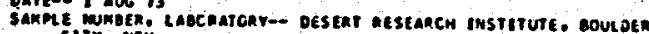

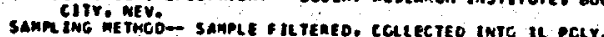

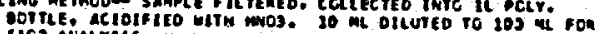

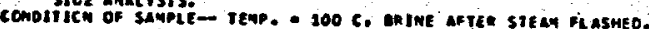
onsica gara

Sp. ecnouctance- 2raco.oe nicromosica ret Diss sxitos 10691.00 MG/L. Sun enine oura

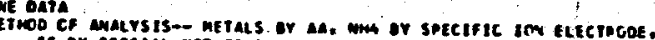
SE OY SPECIAL meTHOD IWVOLVIMG AA, SEE SANDERS AMO HILIS untris- 1976 !

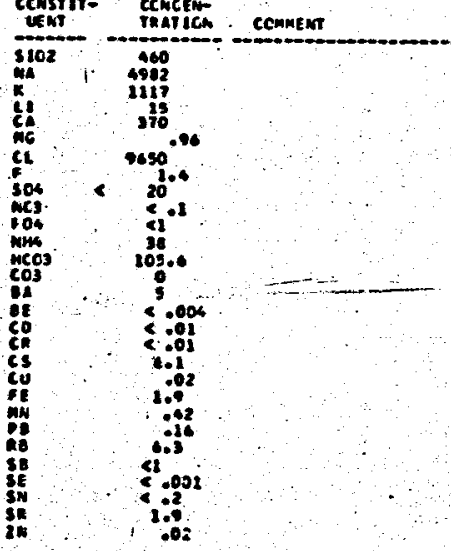

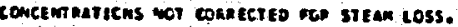

orectosinemic ata sources-

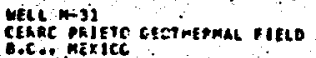

\section{Metciko iis}

CCDE MAKE-M-3ik

Sambiting infotmatrew

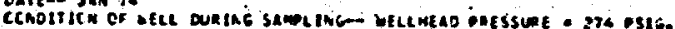
arine oure

vitts- - ON

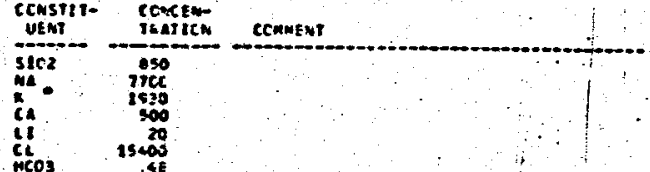

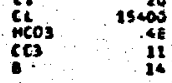

etgl lographic gata

SCURCES--

\section{Aecero 210

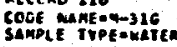

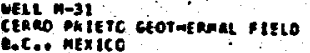

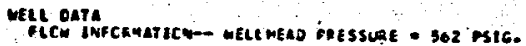

Sanplase zmFcanarion

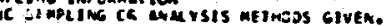

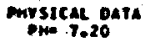

earue cata.

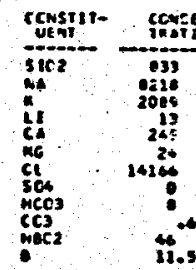

averace amairsis

negclegente onte

actina to 


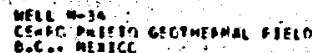

Metivara

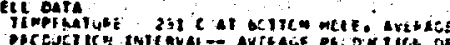

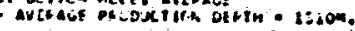

sappizac thichantice

onsient onje

cemmentio. on calculatio.

onsing onls

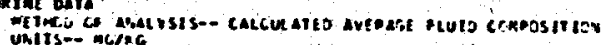

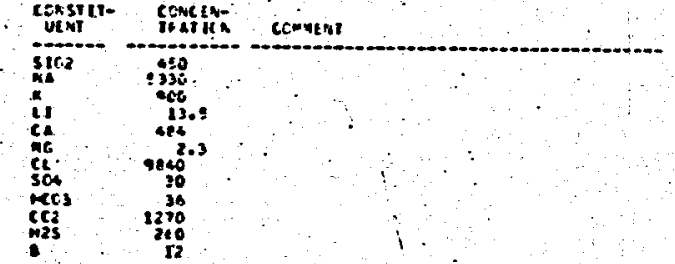

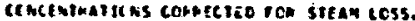

ergz mocasputc pata scutere is

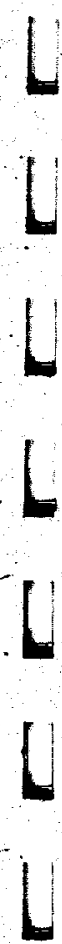

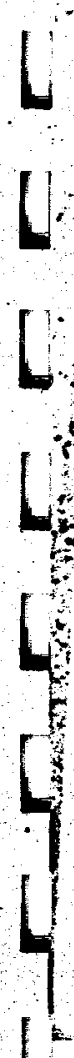

aecono 110 CCDE RAYE EN-343

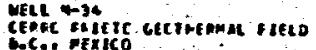

meci onis

Itpproatuse des c at strabatce.

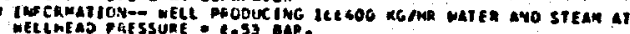

sampine insonnation

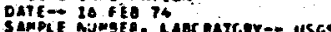

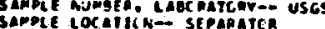

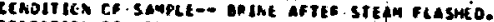

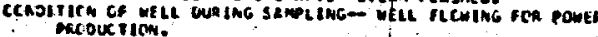

oursicue oate

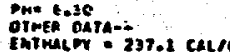

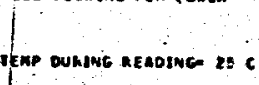

cuine cata.

crisili-

consint- cencew-

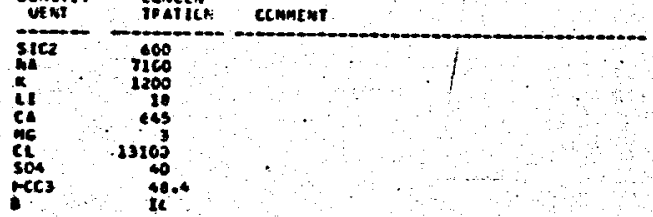

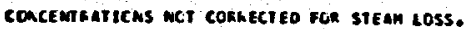

alsciogeaputc oata

serefesto

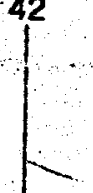

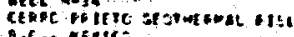

e.c.. exicic

sauplate ivyosimatien

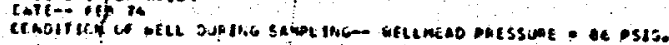
orine oure

unitson- on

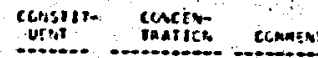

siin.

sire

ce

il

$\underset{c c}{n C C} 3$

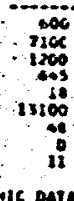

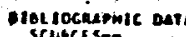

nencado is
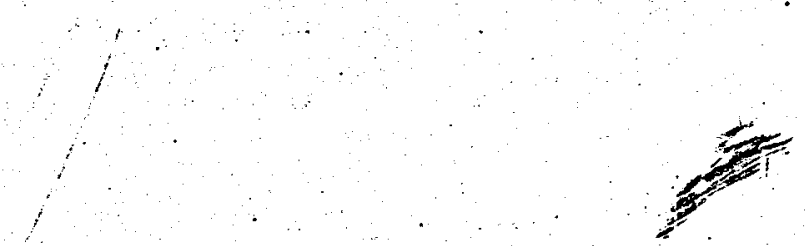

aecono 120

CCOE MAME AH-3 40

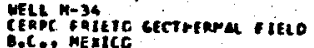

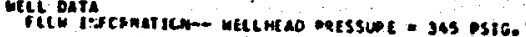

Sampa ing tmformar tom

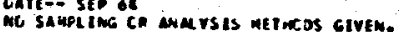

Prysicat oarts

MIME Data

unt is $-=$ pon

constirt- cowcen

Jasilon Connent

sic.

is

c1 120

MG 10202

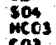

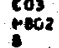

oloilographic outa

Sources-c. 
ecceno 128

CoeE hentem-sga

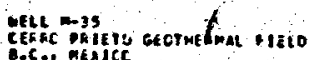

wele informalich

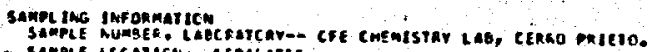

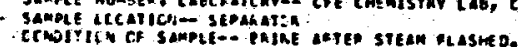

omsical oale

ICI diss scil ose iesce.ce obing sum

onime para

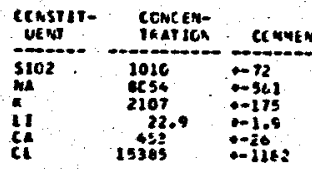

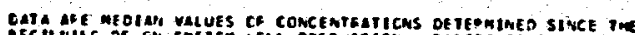

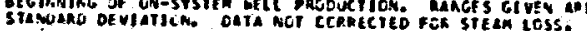

- igchocrapmic data

scinces

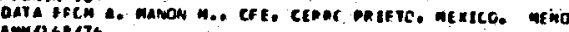

i

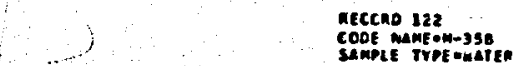

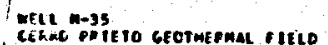

1.8.c. mexice

meLe enfogmation

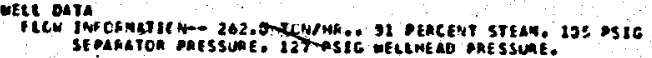

Sann the Troonaralion $-x$

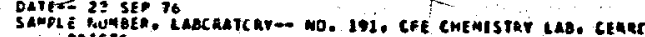

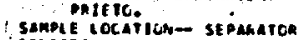

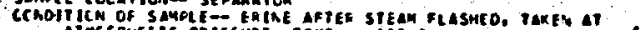

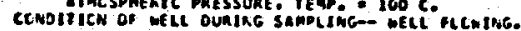

mosical oaya

ici biss schsus. asa12.00 im

ORIME OAIA

CCossuts- concen-

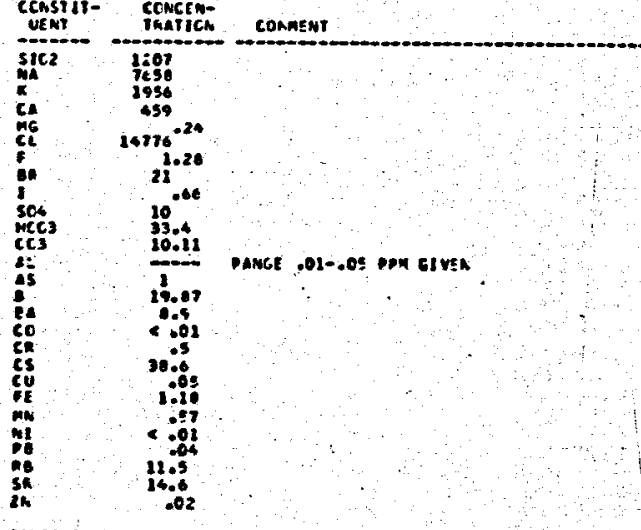

CCRCENTRATICNS NCT CCAKECIED RCR STEAM LOSS.

emelicgrapatc oata

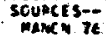

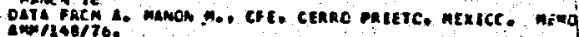

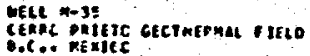

atecre 123

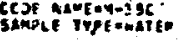

Sampithe Imcrenirion

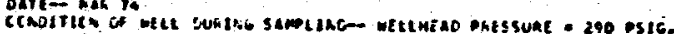
onine oare

unirs-- on

icastiv- torten-

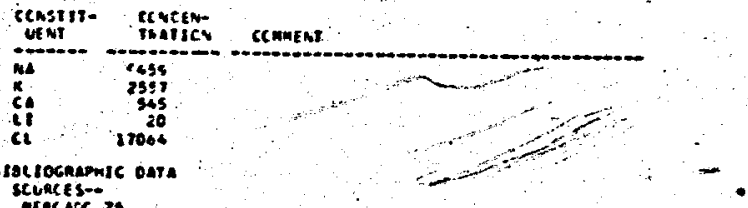

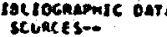

niac ace is

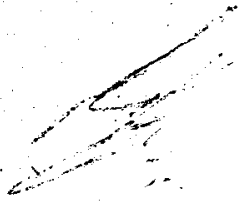

aeceno lat

COOE WKE HE-3eA

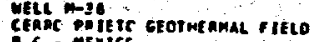

B.c., Mexice

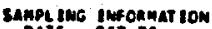

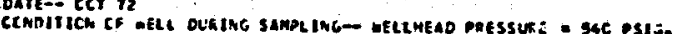

Gezine oaye

CONSTIT- CCNCEN UENT- COMNEN

sto2

ci:

nees

ces?

oreciocrapric onta

MEACADC is 


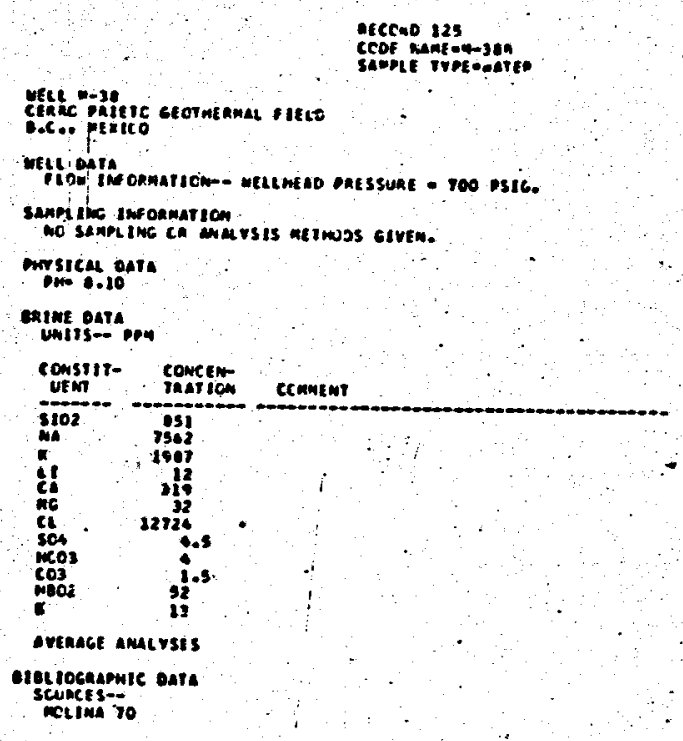

etcono $12 \mathrm{c}$ COof manten-3os

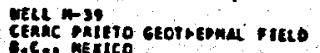

MEL InFonmation

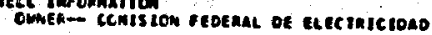

sanplate imformarto

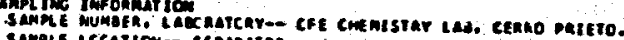

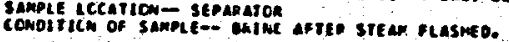

minsical oats

ret olss sollos. 26321.00 com. sum

caime oara

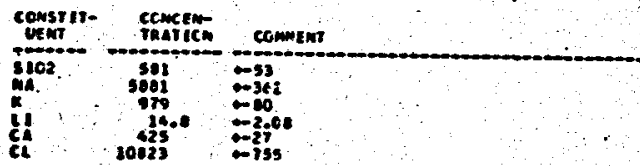

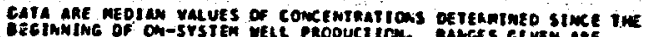

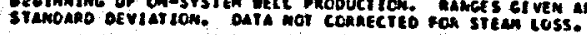

etecjocinapic enta

souncess-i-

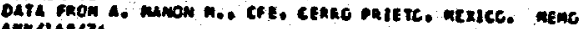

WELL

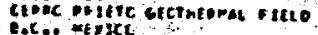

Netu exosention

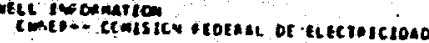

meec cosse

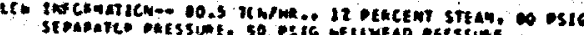

semalinc zeccianarick

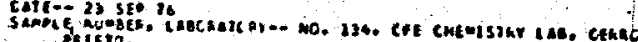
Manita.

sanne cecticn- sepessatio

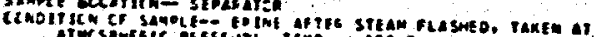

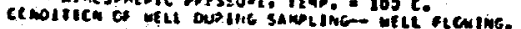

nrsical oata

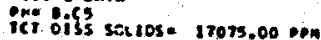

eaine pera.

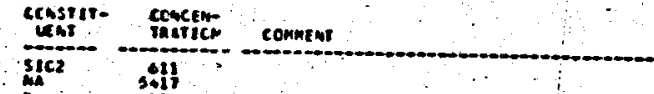

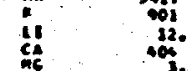

प1

is 20000

sces

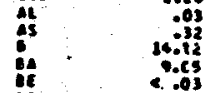

bance cos-ocs men alven

?at

is $23:$

if

If 20.0

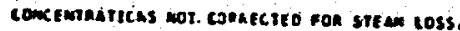

tet rocenputc oata

scurces 70

maxeryo

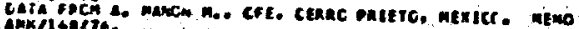

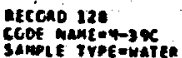

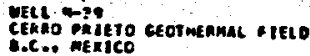

mell oaya

TEmperatuke 240 c at ucitcr more ayenace

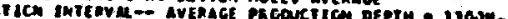
Sanprive imoanaritin

sampet munben, Lackentav-- uses

Ming ouy

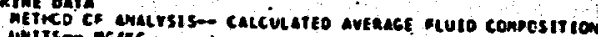
unitis- mare.

crinst1'- concers

vent

sic2

$\min ^{2}$

i⿱

ai

$\sec _{1}$

Thention

ecrnetre

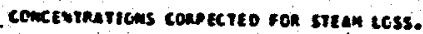

eresiccrapmac anta

SCUKC is 
atcono 129

Cook wank $+4-360$.

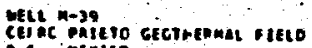

Coc.o. mexico

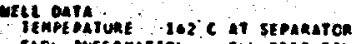

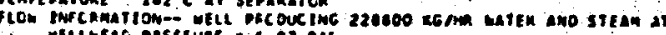

sampiting insonmazion

Date- da ite 7

SAmse Auppen. Labcintcar- uscs

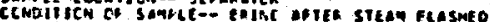

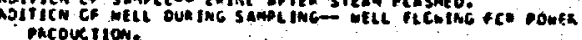

oursical oati

Pris $8.4 c$

InTmelory 254 calec

Jike OUAINE REAOIMG 25

orluk gatra

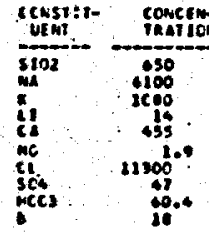

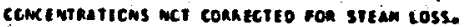

erol locrapmic eata

seviectes-

\section{atcend 130}

coct manten-39E.

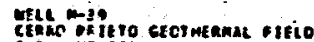

E.t.. infiec

sampling impormarien

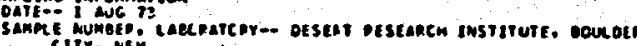
samsi enc

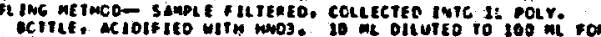

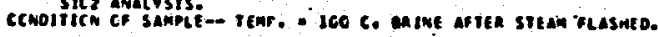

omsicas oara

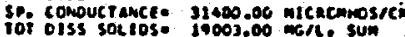

MajuE OATa

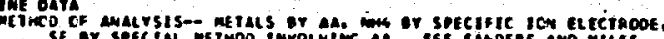

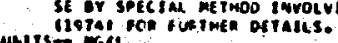

censul1. concero

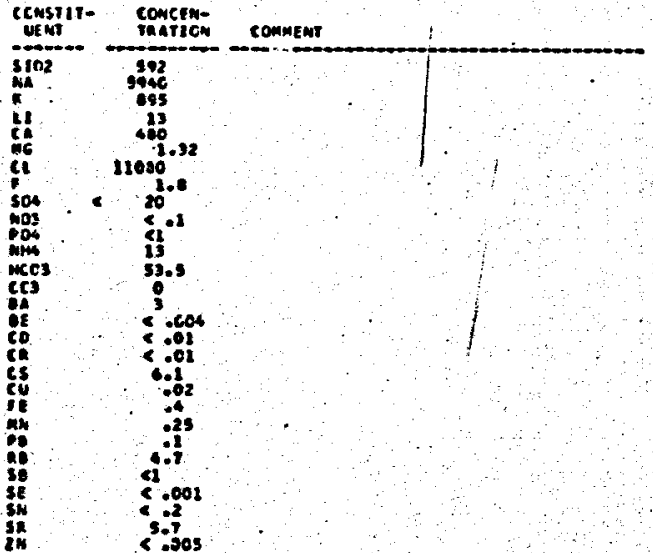

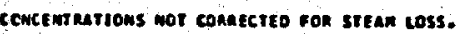

etbirocramic oate

Schotés 74
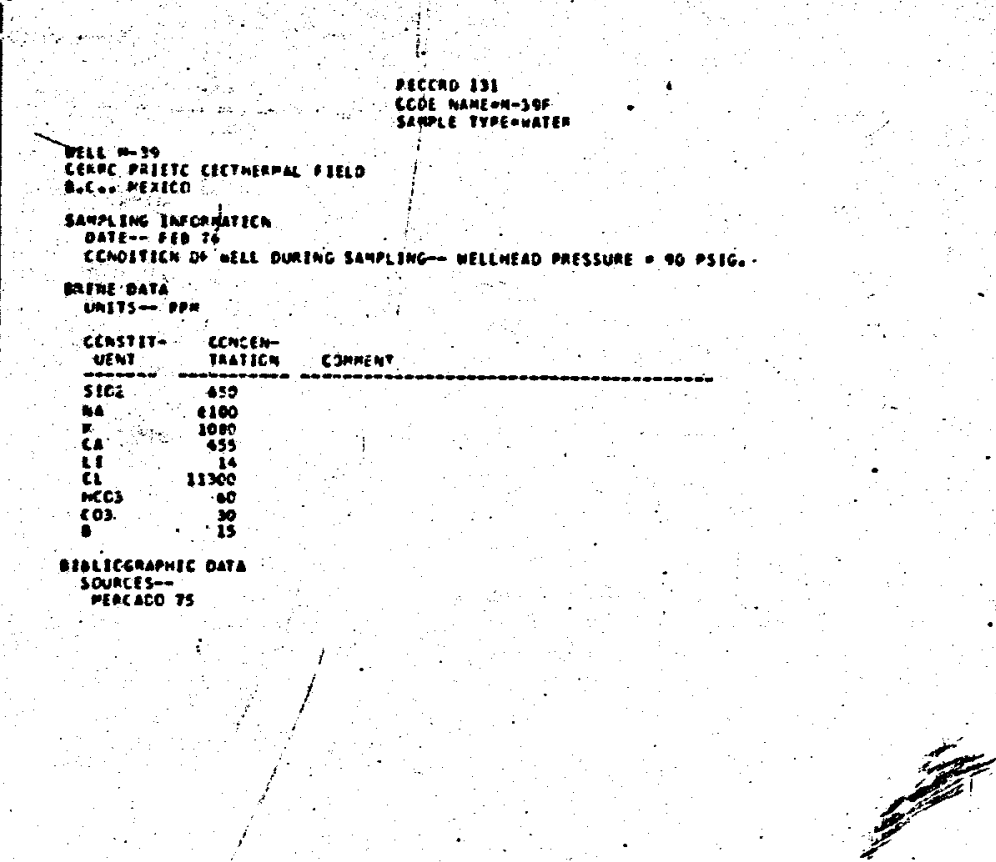

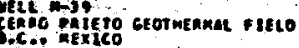

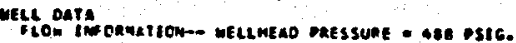

sann ghe infonwer scm

no sampl ing on anatrsis ne twos given.

omsicat pate

earue pata.

Ualis-: ton

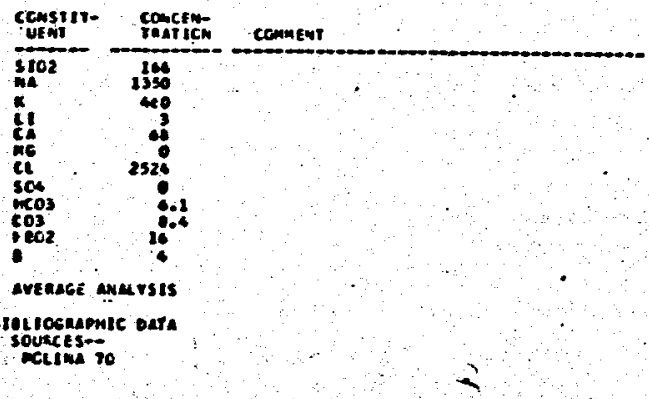

afcono 232

CODE MAMEOM-39G
SAMTE TTOE OWATE 


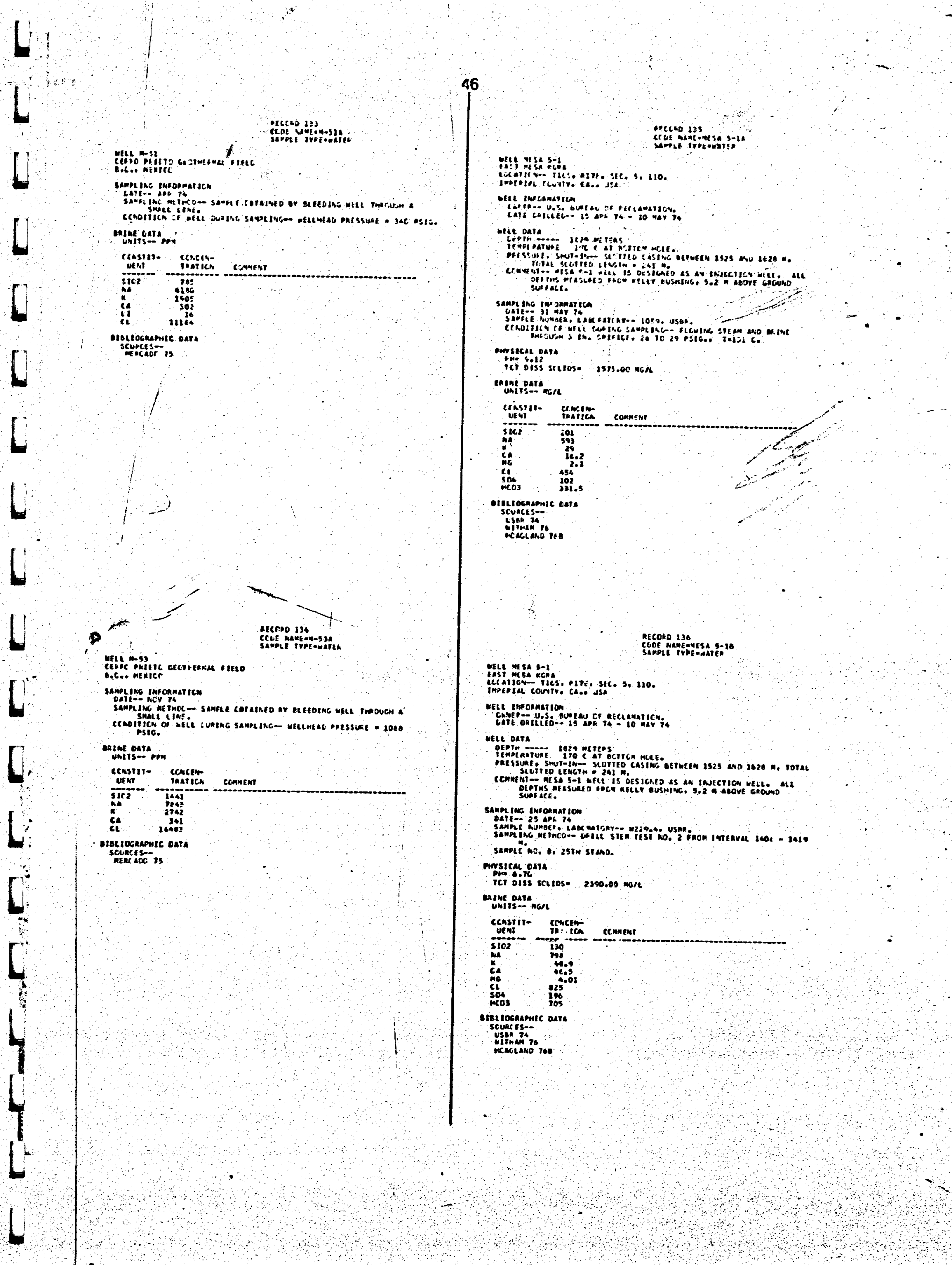




\section{ALCLED 137}

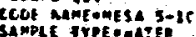

Eeti nest $3-2$

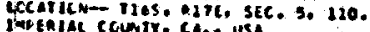

The ensormation

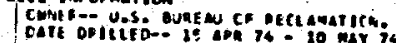

met oira

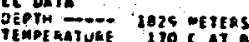

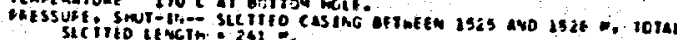

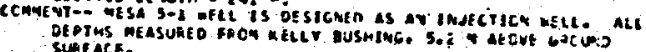

ShMplimb infoenation

CAlt-- as aps 16

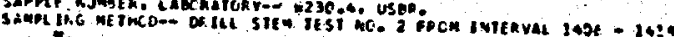

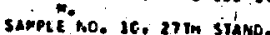

onrsical oats"

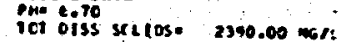

eximf onye

uniss-- nagh

ccksrito concen-

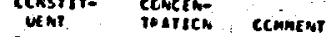

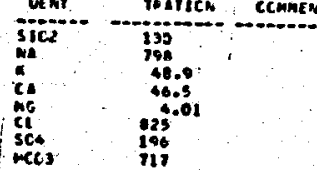

mes

mol lograpmic doata

ustan 14

Genat 16

\section{Aeccos 13}

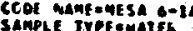

Katl ness e-2,

CaAtil

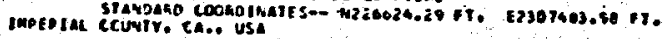

vele turobution

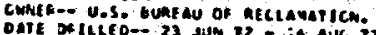

metions

JGePreatuet 2448 WE TEPS

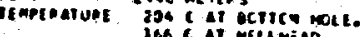

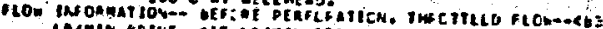

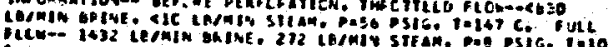

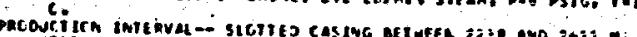

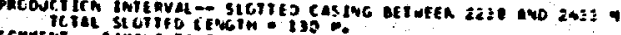

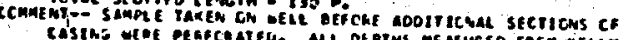

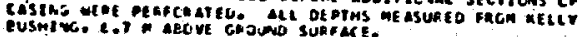

sampiline jur aanurjom

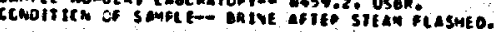

monsical oats

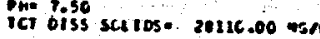

exine oare

Censilt- crinceno

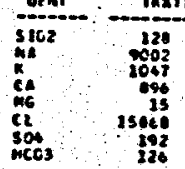

DHELTEGRDHIC DATA

cuser 54

Himin 10

ACACLAND 36 math

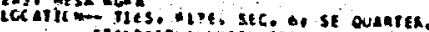

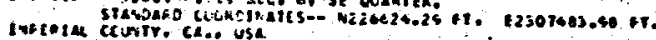

- fel infcamation

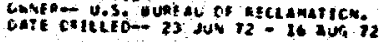

Wethonta

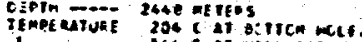

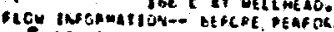

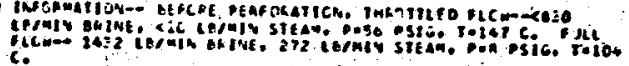

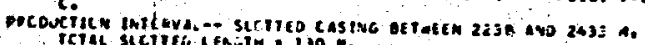

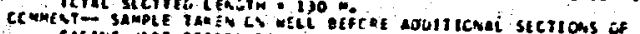

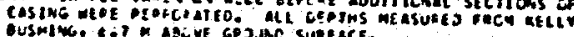

segrige Imokmagen.

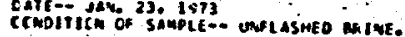

minsicm ons

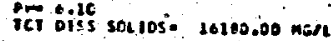

earate oart

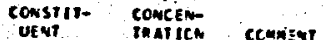

$31020 \frac{220}{2129}$

8129
42
469

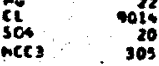

eroutcocreamic oara

scuptis-

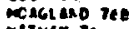

Himas 7. 


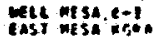

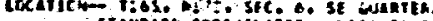

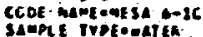

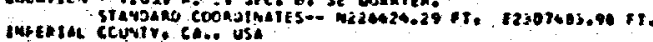

MELL Imotimation

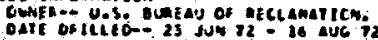

mete oara

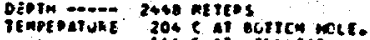

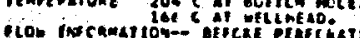

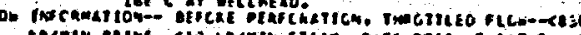

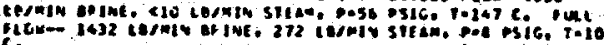

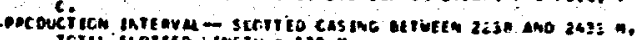

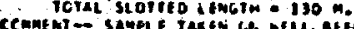

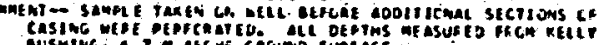

ine imosenation

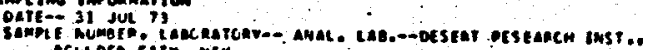

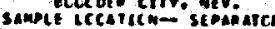

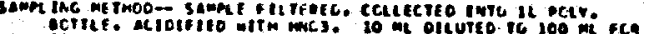

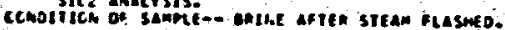

ongsicetionte

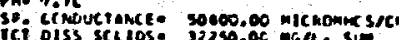

MnME Date

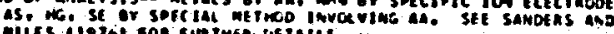
umirs- miles:

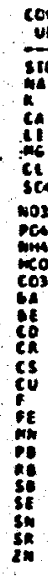

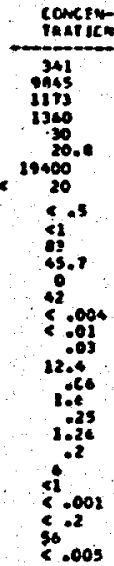

cemanent

$[1$

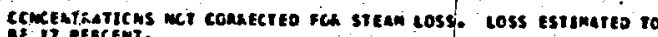

gozrocramic oata

scuaces

SaMOEAS
WCACLANO

milman 7 tats

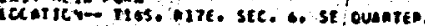

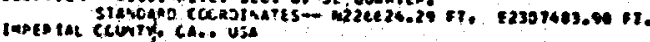

ntic imonentión

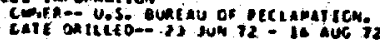

uftet gara

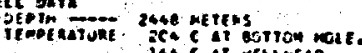

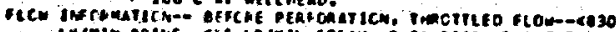

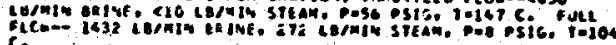

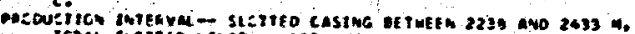

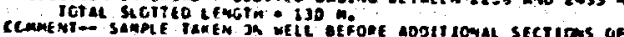

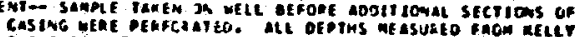

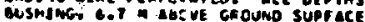

senpelate intornariom

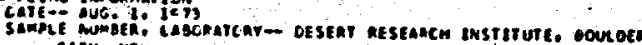

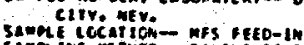

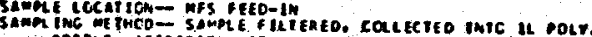

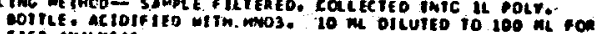

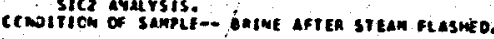

omsicar oare

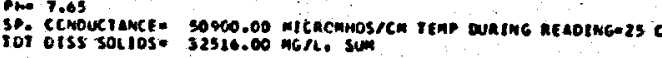

mine olis

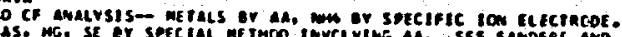

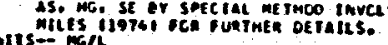

ceas:I1- ecuictin-

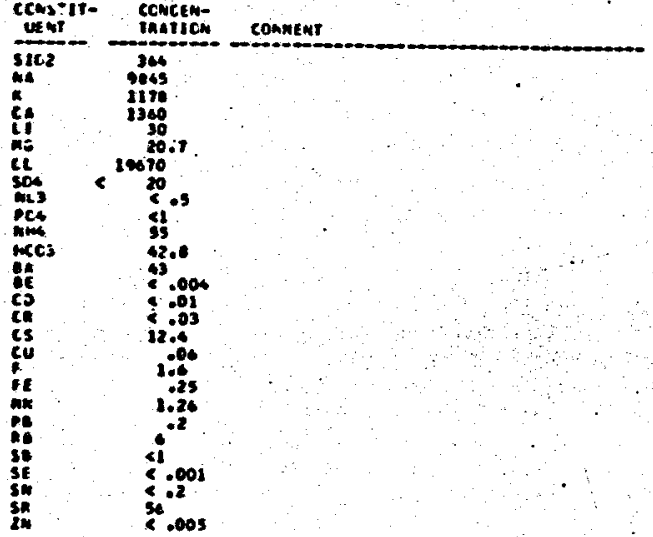

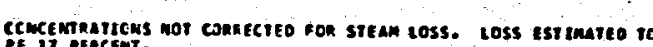

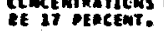

sugizccaepric data

sanders 10

climan is

ofi is. 
Fat Mest e-1.

\section{atcono 148

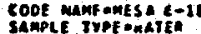

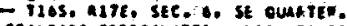

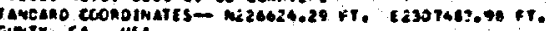

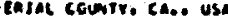

wete Inogmairion

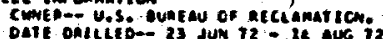

vete Data

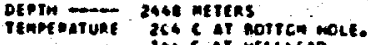

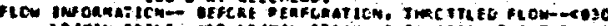

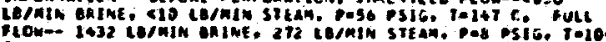

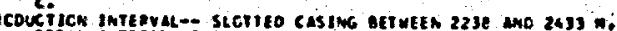

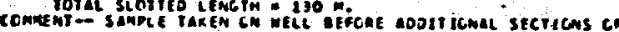

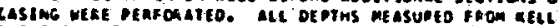

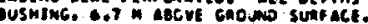

SaMPl IMG InF ORMATION

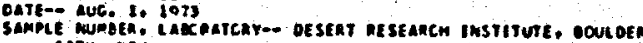

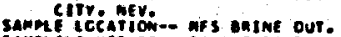

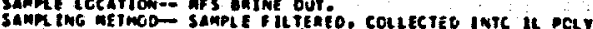

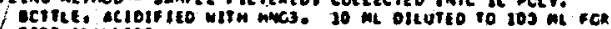

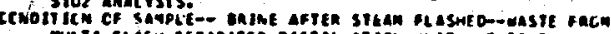

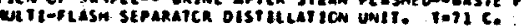

owrsical data

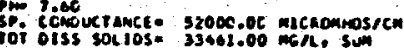

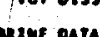

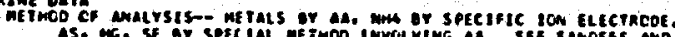

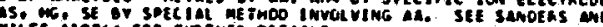

unitso-

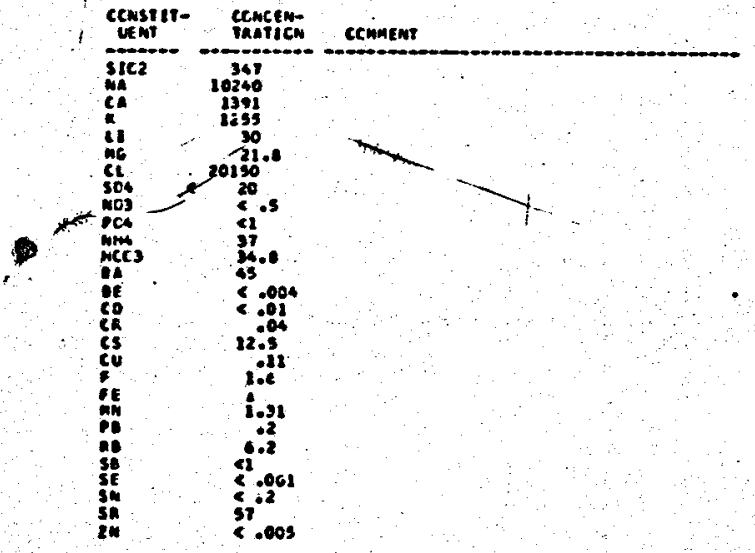

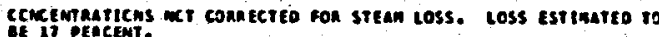

otelsocmaphic oate

sampens

intmat is.

int 34

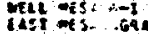

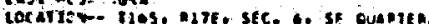

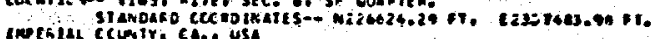

ieli inopratsen

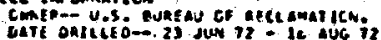

mulgata

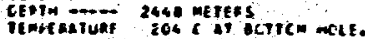

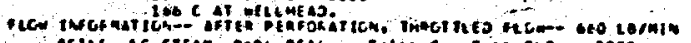

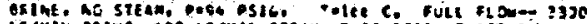

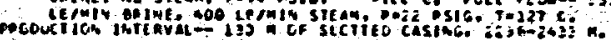

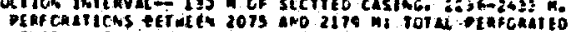

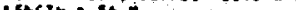

crment-- Atc Deping chouns sunt act.

simpitue imponatice.

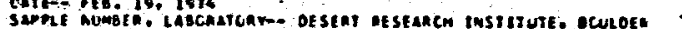
CIIr. his:

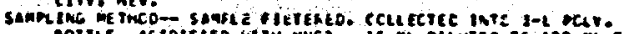

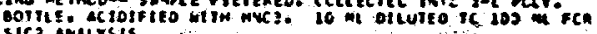

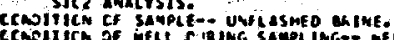

mersicm oals

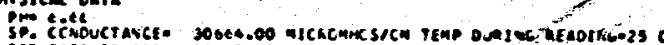

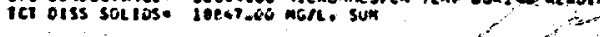

carme care.

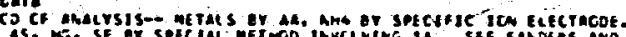

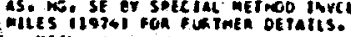

units-- Mas

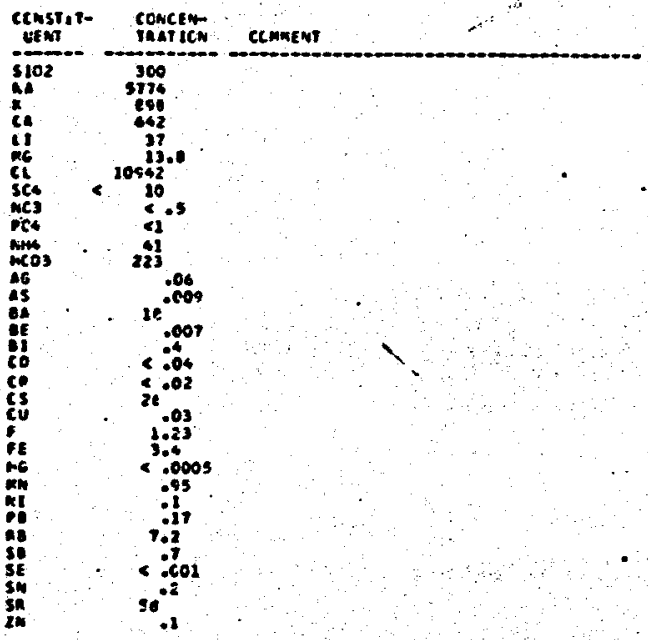

SAMBRE UNFLASMED.

esoctrockenmic onts

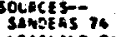

Meafeant 760

Titran 


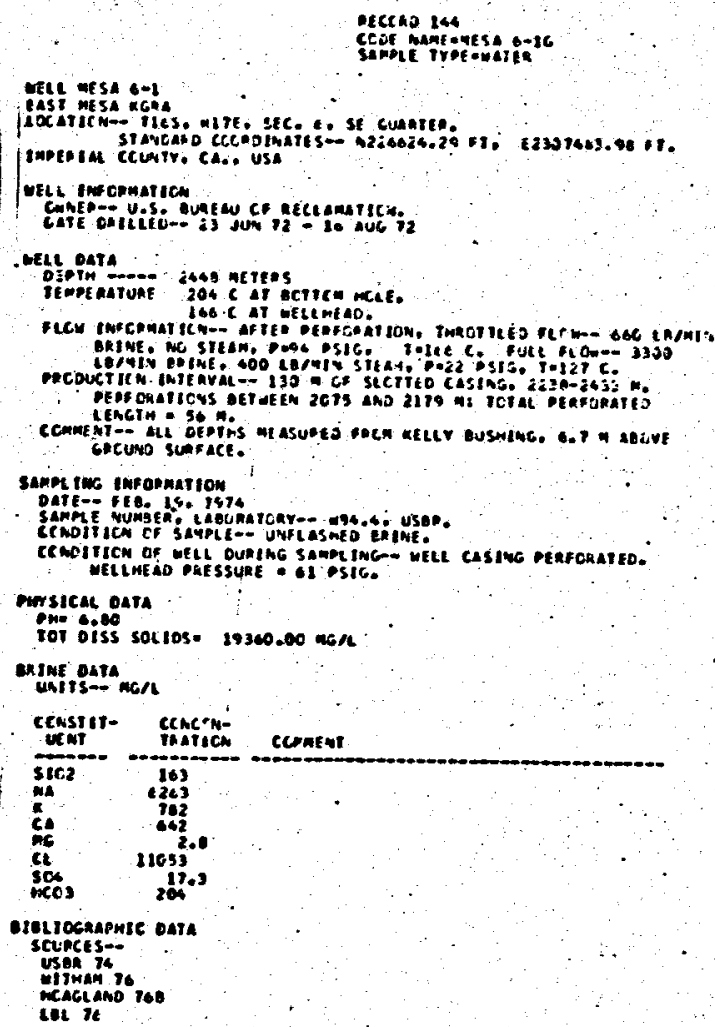

necrino 144

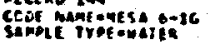

mete mest

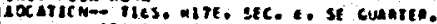

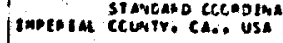

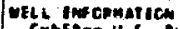

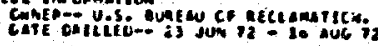

cheze para

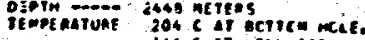

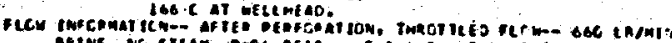

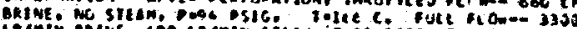

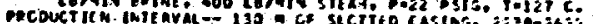

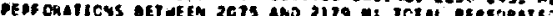

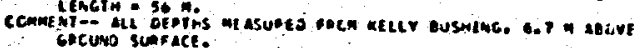

Sakpe two tmposmation

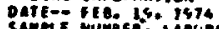

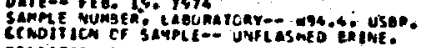

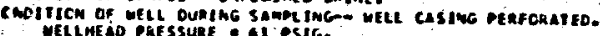

mersicac oara

Tot oiss soliose $20360.00 \mathrm{~min}$ :

units outa

ergerocapamse oata

ustinces jo-

meachano 700

cil 7

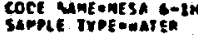

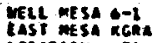

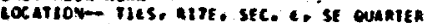

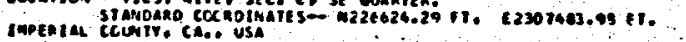

metc uecremartem

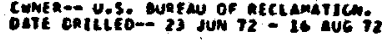

velloara

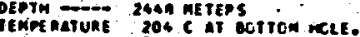

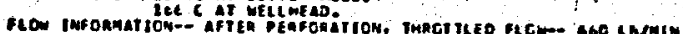

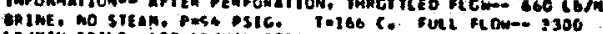

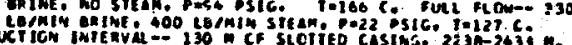

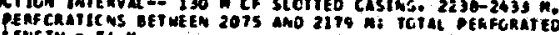

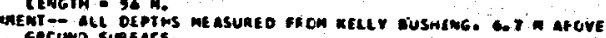

Sanpl inc imformation

DATE $\rightarrow$ JuN. 11. 1974

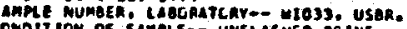

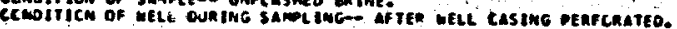

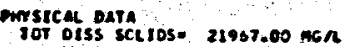

exime oxts

Uaits - MoR

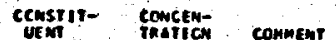

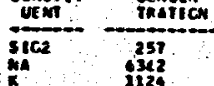

CA 1128

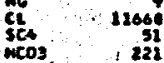

osst rocrenpitic OATA

source 50

HeAGLANO 760

Hit is

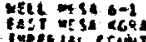

Deccos late

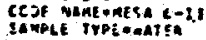

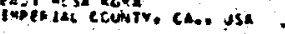

sanptine informaticen.

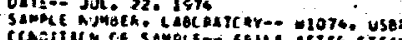

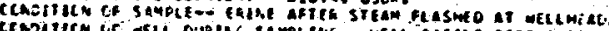

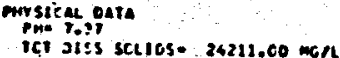

inzenz barta

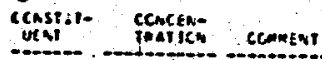

cit 1360

olosacciamic tata

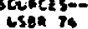

neAchand nes

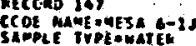

Mate ness has

thitenisi ccourir. ca., us

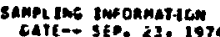

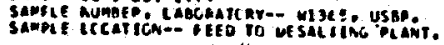

monstcal oara

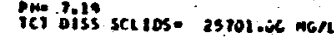

meline eare

clisstr- CEACEN

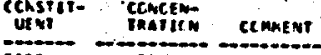

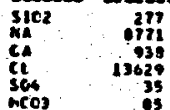

olstiogmemic onts

scuecess-- 



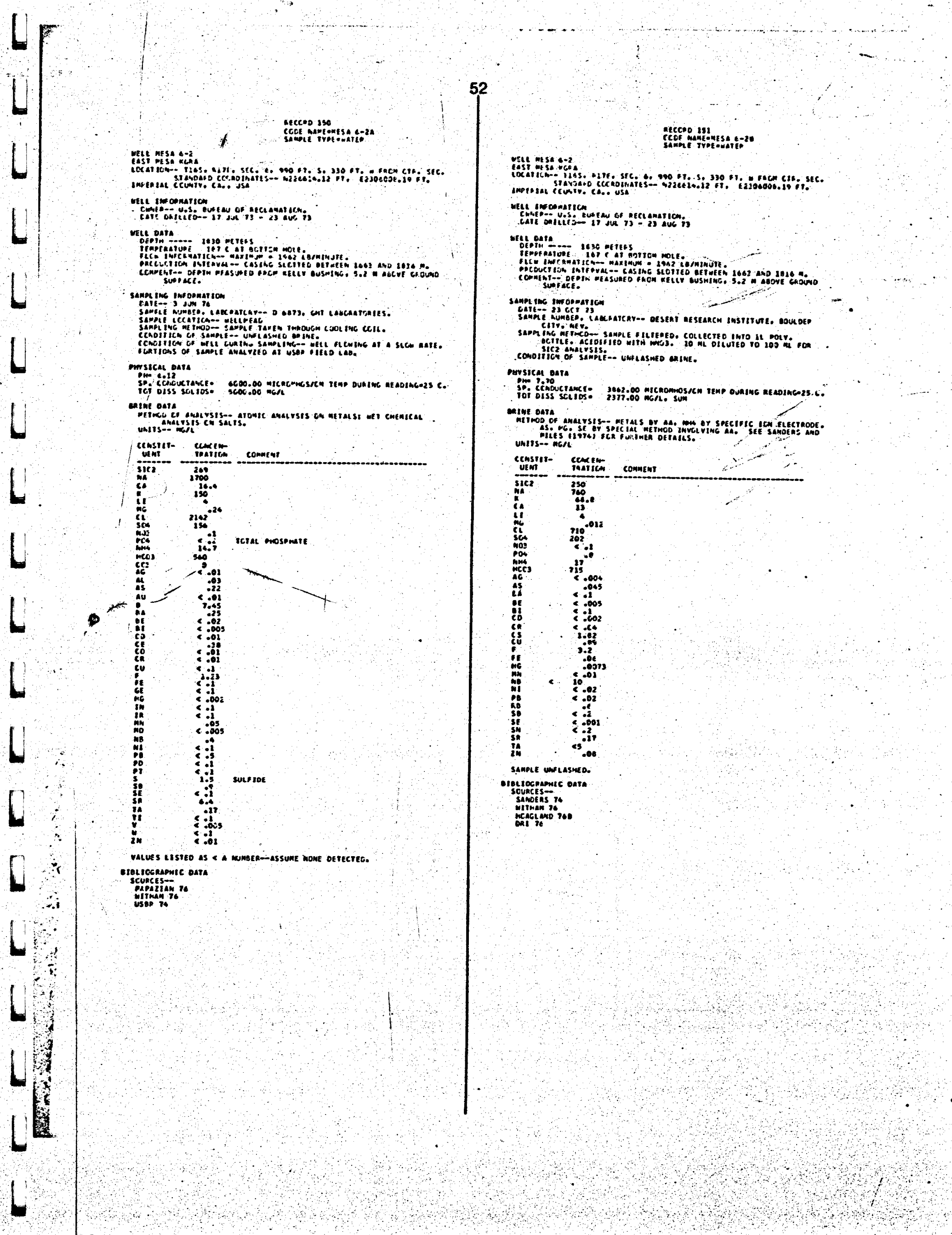


ncono 252

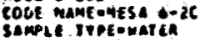

Eets wist e-?.

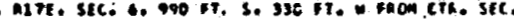
imperial sTanjako coonsimate

WEL INCRation

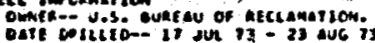

reteris

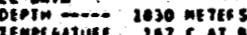

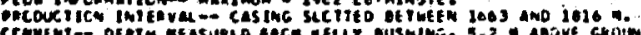
sierace.

sangetim Imonnaticn

oaft- e ug 13 .

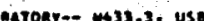

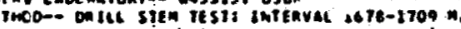

morsicat gata

ICr biss soitoso $2130.00 \mathrm{mon}$

exing cala

censrit- concen commint

otmi- maticm

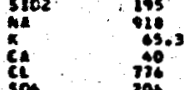

$\underset{1200}{300}$

erseroceremic outa

susece sit

uctrateno

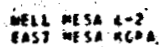

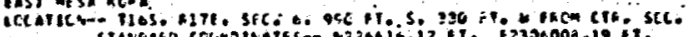

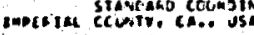

- Ell gircamartón

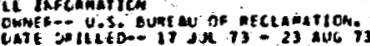

- ozi oata

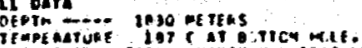

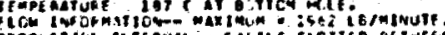

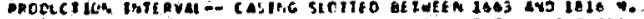

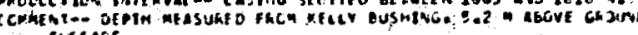

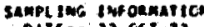

Date- 23 resis 13

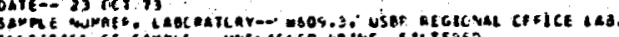

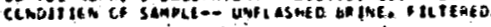

andsecat oars

ict diss sturs: 3130.6e mod

onge "Das:"

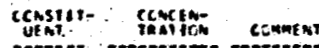

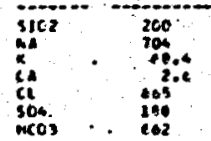

melcocriomic onta

sounces--

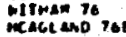

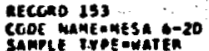

exect mesa $\quad-2$ as

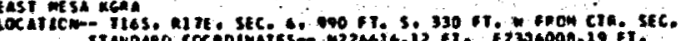
imentar clemirt. Ga.e usa

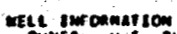

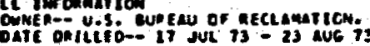

mele una

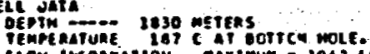

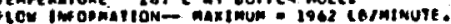

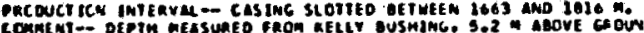

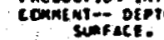

sumpeye ins ormation

DAle-- E Lug 1?

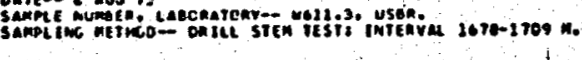

enrsical earta

ICI Diss sol 10S. 2700.00 MGn

aine oura

CCMSTII- Concen

Connew
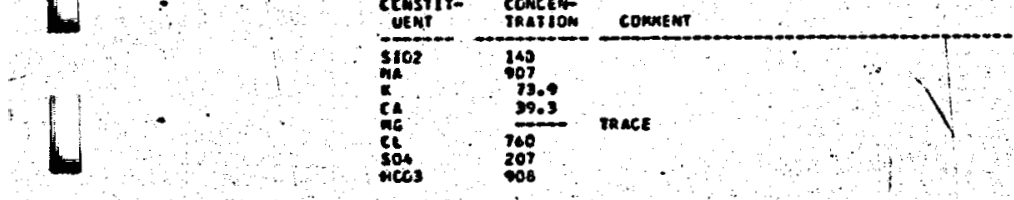

ciectoctupic data

soukct 34

utrman 70 


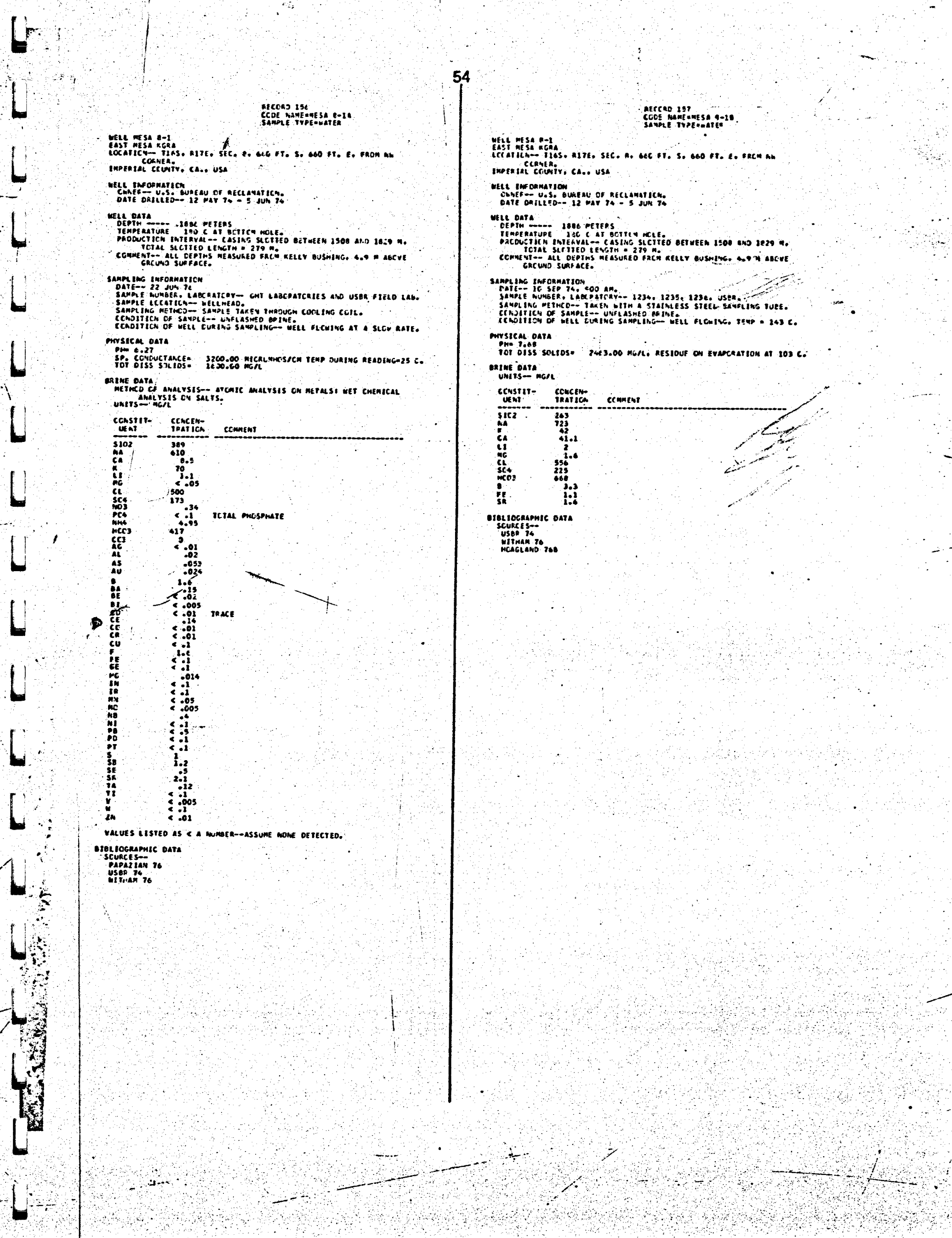


Deccas 1:8.

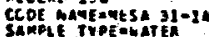

tets yesa $31-1$

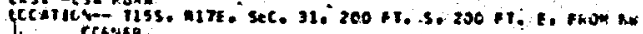

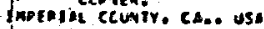

Hell ans comatica

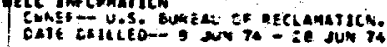

wete care

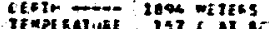

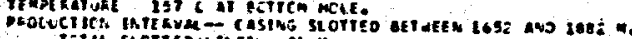

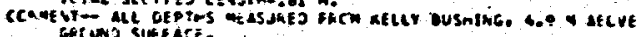

sampliag throphartem

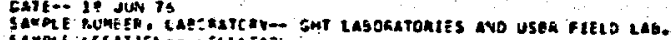

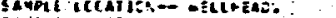

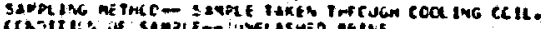

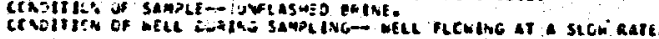
mistcet dats

in o.zT

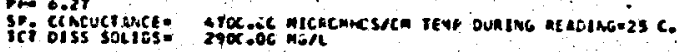

engae oata

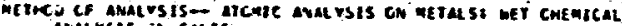

untis inatrsis

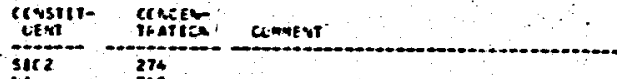

$\sin _{312}^{37 t}$

is

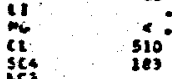

set.

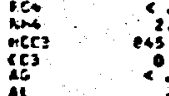

an 20

if 20

it $: 11$

id

iv

if $a_{0}$

it

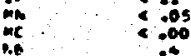

酷

in

se 1

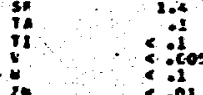

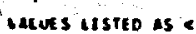

ergeicenaprat oata

iastiain $n$

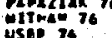

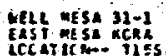

retcro 238

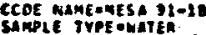

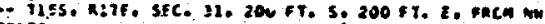
inparial cClniv. Ca.. uss

Metc In camation

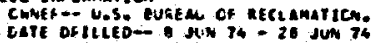

mete gers

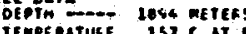

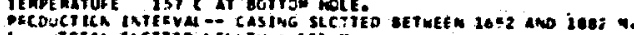

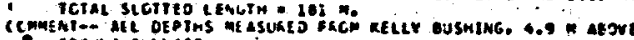
cinu sistace.

Sampling imformation

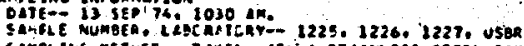

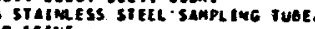

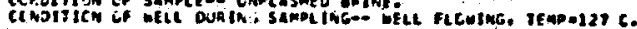

Derrsices oata

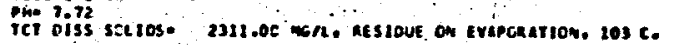
exIME OAY

UNITS-O MGS

SICE

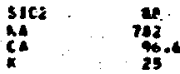

it

${ }_{\text {uct }}^{4 c 5}$

if $\quad \begin{array}{r}2.2 \\ 2 ; 4\end{array}$

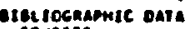

toster 76

mitman ie 


\section{Aecono 160 \\ CCOE MATE HEAST nesA $16-10$}

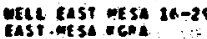

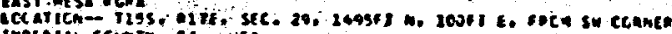
impleial cruntr. ca... use

WFil in onmation

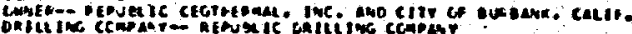

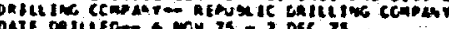

meitipare

Gipin zins meteos

42 c 437 metres

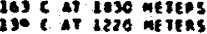

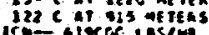

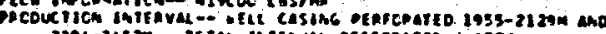

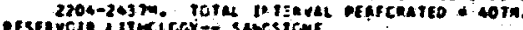

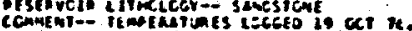

saxpl tuc insobaniten

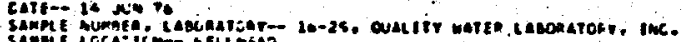

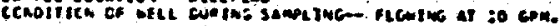

morsicas oars

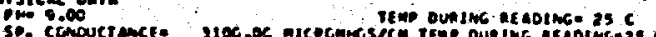
(at

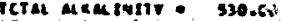

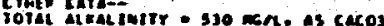

tojue oure

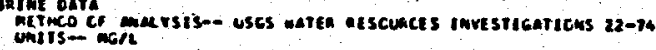
ctusiti- cancin

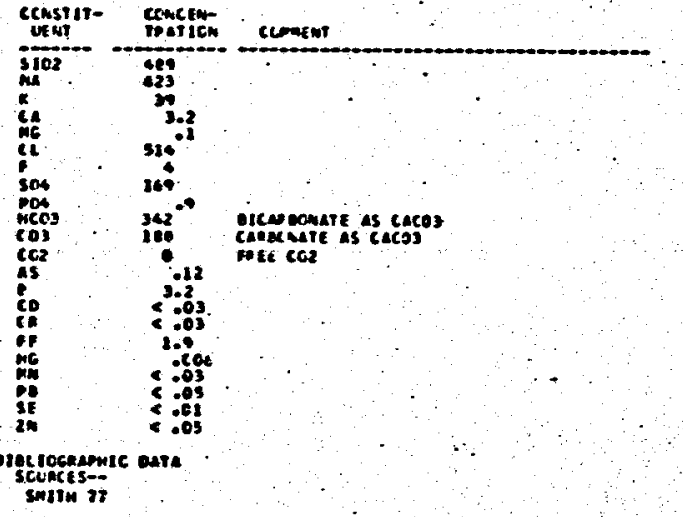

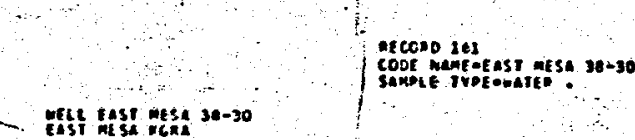

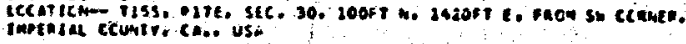

wete inecosation

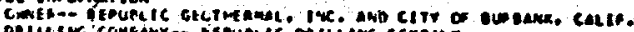

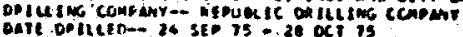

cil onta

Iempreatuat 2740 metens

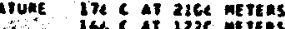

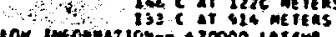

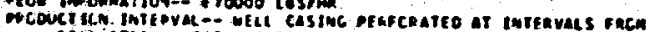

104l-2 rizn. Totel thienval pearonateo - gston.

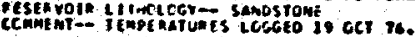

sanet Ine Infoknation

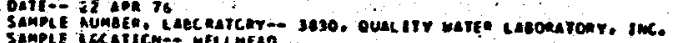

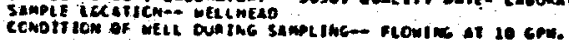

omstcat onta

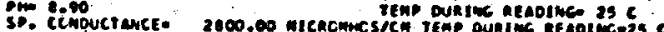

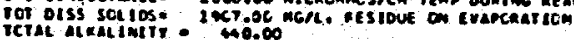

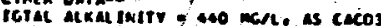

calwe outa

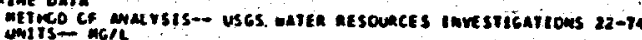

Consint- concen-

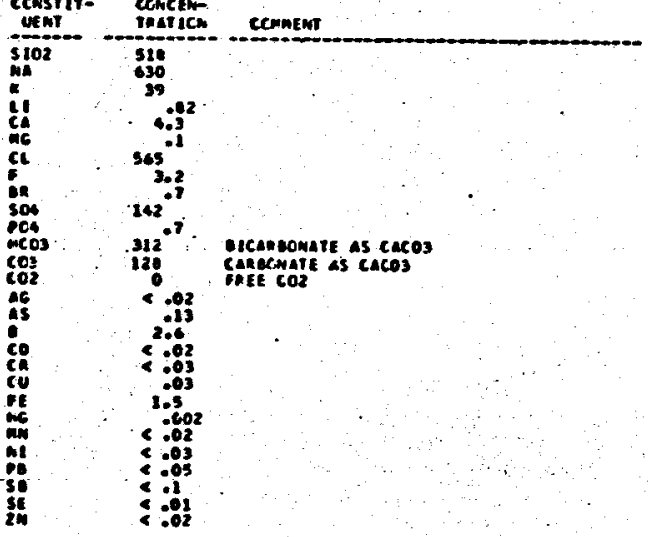

oror socrentic oata

SCyntin 


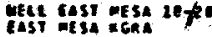

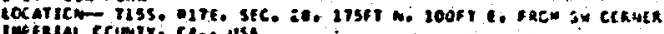

interial ccowir.

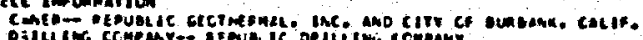

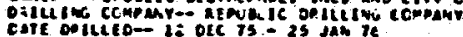

veli para

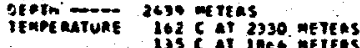

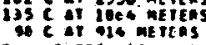

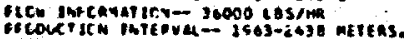

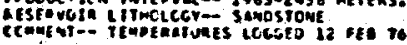

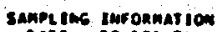

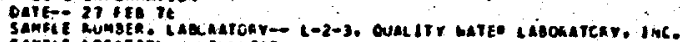

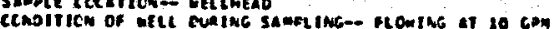

nersical cata

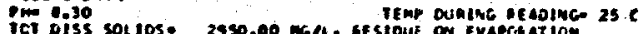

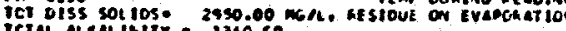

CTM AxM Bhity. 1340.60

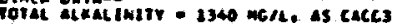

caine oata

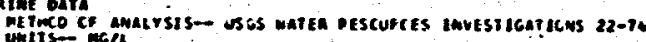

Ccustrit- coneto

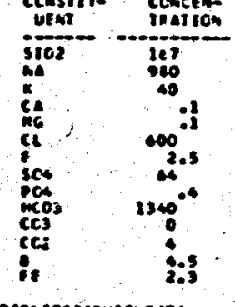

etersocinomis oata scuaces-

Cominer

Gicangcciate as cacco

gate COZ AS CaECs
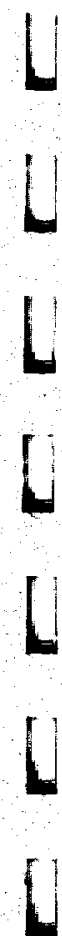

200020263

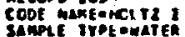

Mat acinz

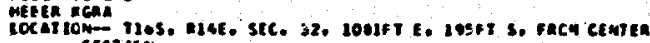

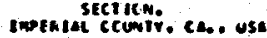

meti liscanatzon

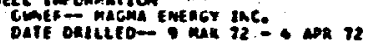

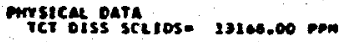

onimz oare.

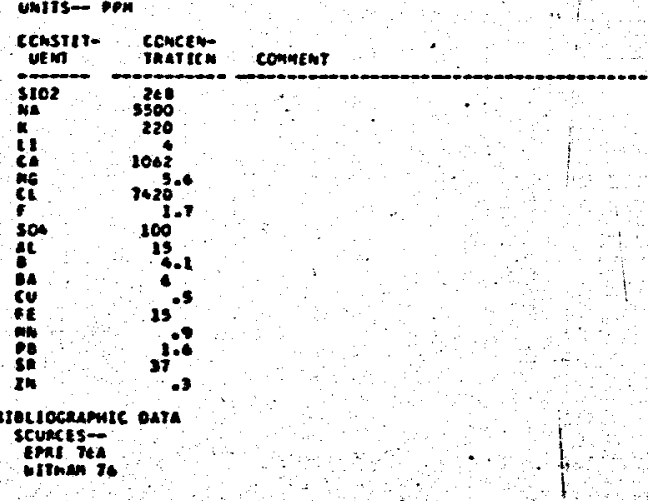

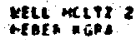

Afceno 124

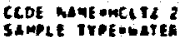

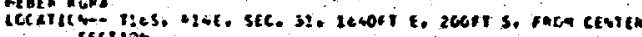

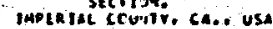

Hele In crasision

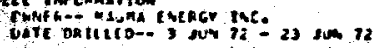

onvsient óte

ict ciss saltose les3oice on

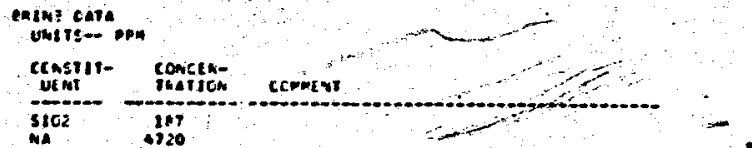

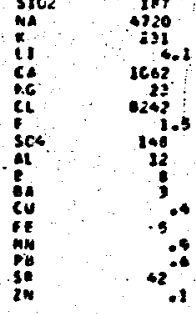

- ing tocksomic data.

iphe

iminem is

etcono 10 s

CCok Mateces. Jacksch

tele to. Jacascon

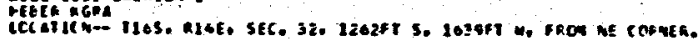

wetl Inf caratich

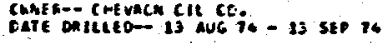

mirsicas bats

iet titso sme

oneme garse.,

Consilt- concen-

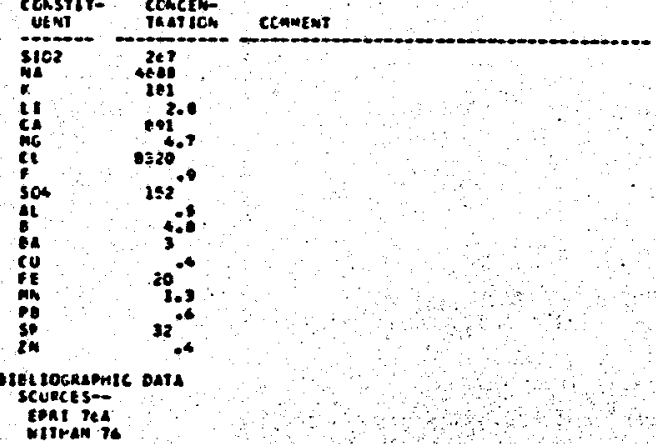


areris 166

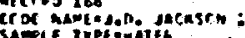

MeL J.o. secreson 1

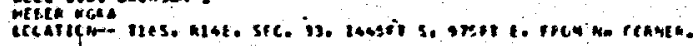

veli inf comaticen

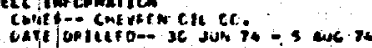

onsiche oata

iri oiss solicose s5218.00 ing

caine cati

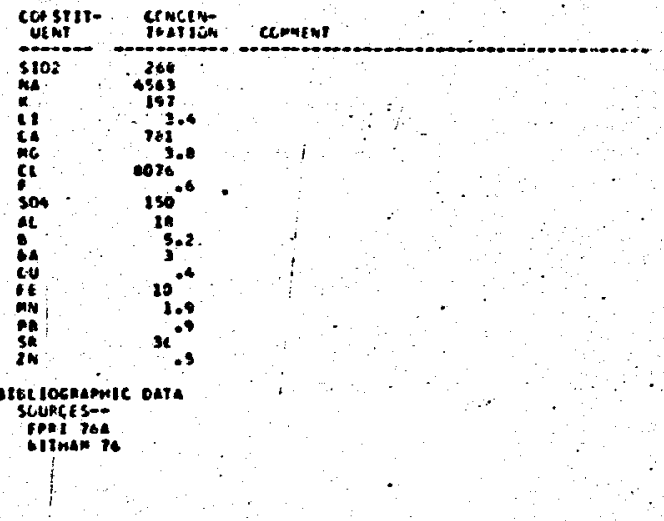

aeccos let

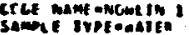

mete Nivin 1

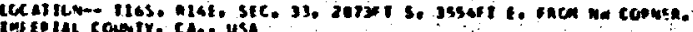

MELi Iwoonanion

mete oara

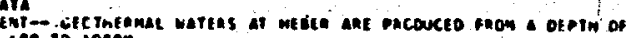
cos 10.200000

mersicas onts

icl oiss sculos. $141 \times .20$ nom

unine pala.

censist- vinciso

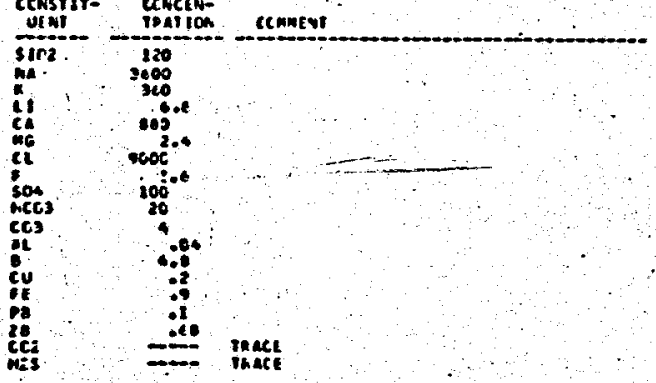

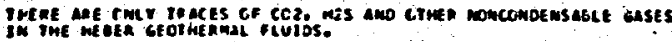

sistrogenoutc ours.

sources-

\section{Dícero jee

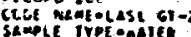

Meth cast bet-2

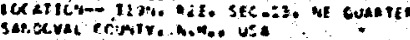

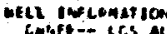

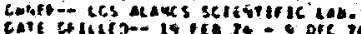

vels oate

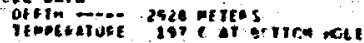

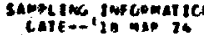

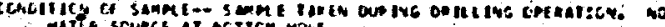
mith scurce at acricm mats.

mrstcal data

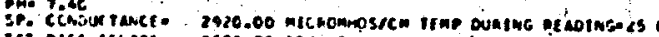

ici oiss scliose 2300.00 ppr. sas

torat manomess as caros - ito pou

earne oara

uncis-pon

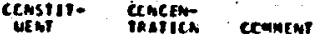

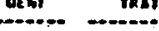

Ci.

C. $\quad 100$

3063

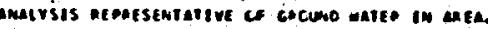

etociocesmic oate

suments

miman $x$

cuntruming

$\lim _{101} 536$

iinition

otwek motes

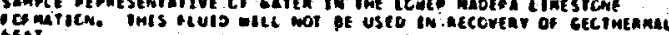

Ereono 169
COOE nanfocmance

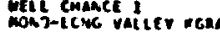

tocaten- iss. pzaf. ste. so

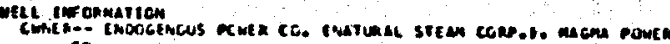

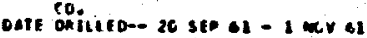

okjue oape

ergyocresmic oate

$x$ Alit 63

mitmen 16 
with thociencus it

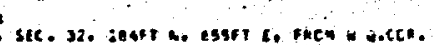

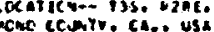

mets iwerinatich

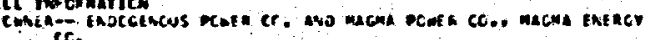
cate ofilcen- 31 du 10 - ir ave so..

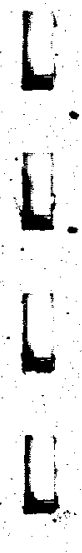

vele ont

Devir 19 a milis

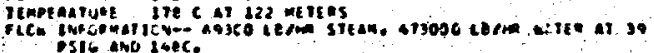
siwizing insonmar tee

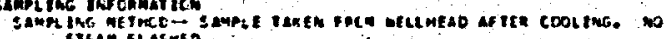

omsical pera

on e.e.

exine oave

crasill-

Consyill- ccosctive

Leni

$\$ 1 \mathrm{si} 2-230$

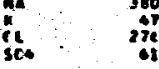

sion iccenpicic onita

scuecerit:

ofCclio 111 .

CCDE MAME EROCGENCUS 20

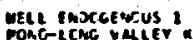

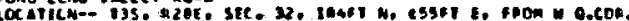
aher centr. ca.e. usa

WEll Iuromarion

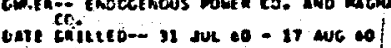

vile para

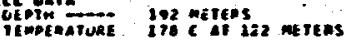

sannsus infonmalich

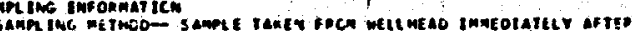

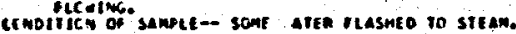

omistcal $0 A 1 A$

Units on

CCNSTIT- CCNCEN-

COMNEN1

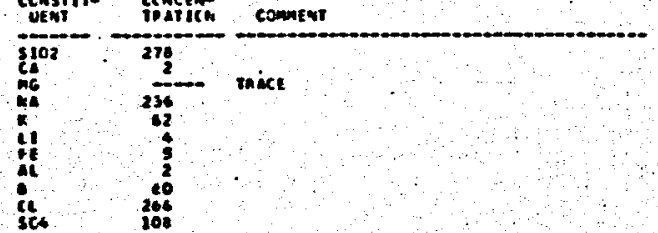

irellograpntc data

simention 


\section{iccoas tis}

ccot maketenctatncis

wete ensccencuss

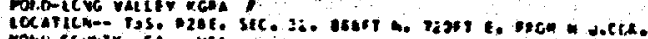
ronu Clumir. CA. Us

Weic Imeopharion

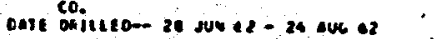

inct oura

eniwe onta

evalisbil.

oteriockanmic oats

scunction

inimen 18 aectano in:

ceot inatetrocelmours.

wete endocemess.

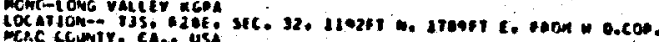

vell imonmatio

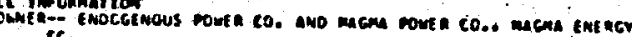

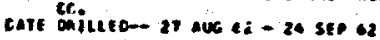

ofoin

anive oare

-iac locknowic oata

scurcesti-
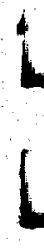

1

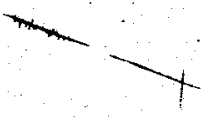

nercre $3 n$

Coof munetwoctnous ?

Wele Enocenous?

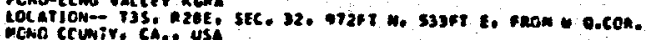

well infonmation

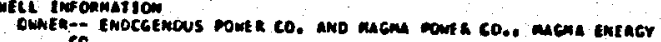
eare ofiliteo- 24 aug $22-25$ set 22

Wetcara 200 mertens

DRgne oute

no gerwe pata avalcable.

olor gocributc oate

nC MIil is

witman is

\section{an

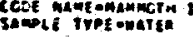

ofectos ine

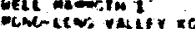

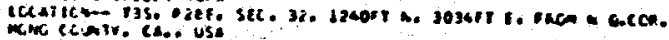

veti incremalices

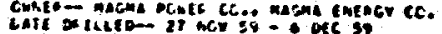

wit oava

240 ortens

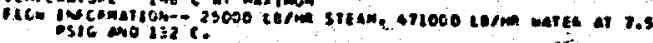

samplese incoenation

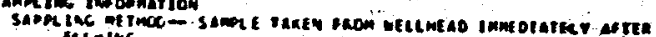

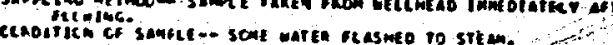

onstca outs

easme outs

vits - on

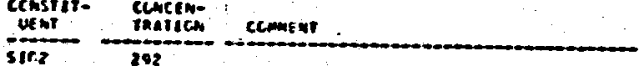

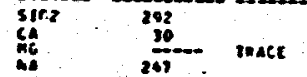

a.

it

it

sor.

II

30

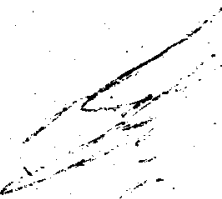

inijocenmutc bata

Solrcestin:

wetl 37 are me $\$ 397.1--1$

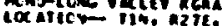

acno ectirir.

weth Incentio

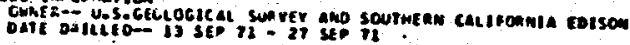

vet oata

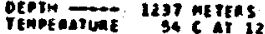

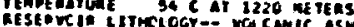

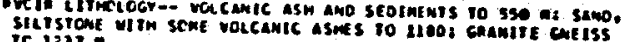

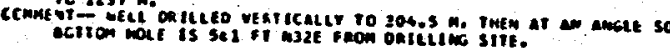

oxine oats

cars avarleace.

electrocknonic oara

scuinces-

Axitex 72

WITHen 70

arcene 100

WeCe stere rac asmi.1--23.2

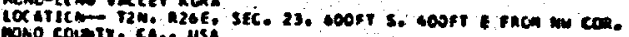

urit incourtion

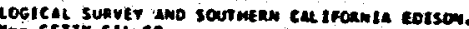

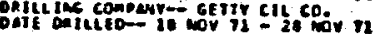

meti para

PEPTH Tat metens

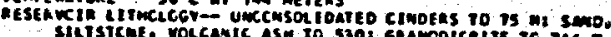

Mine oate bata avaliabie.

mezlocramac ears

scuices 70

axiet: 72 
ecceno in

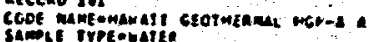

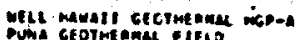
pabati comive mavalt, is

teter incoumarion

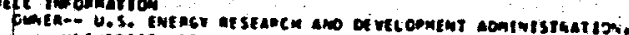

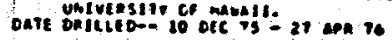

weic onta

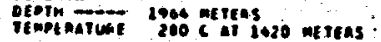

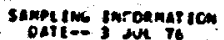

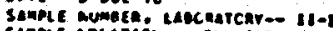

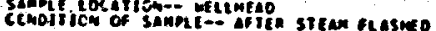

onsiscal vate

ename pare

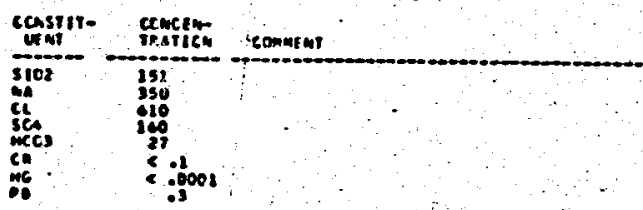

deren strian reasued

eses rockupmic oata

scunces $\rightarrow$

\section{- eccono 202}

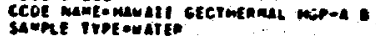

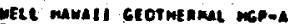

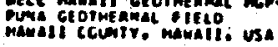

sampl fuc incosingrion

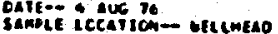

emstcal data

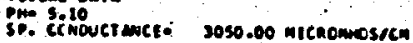

elme oara

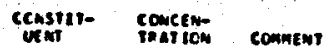

cil

relicaremic data

soupes in

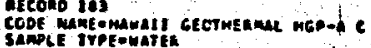

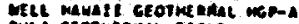

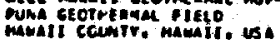

sampe Ine enfoumation

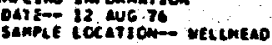

enrsical oats

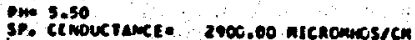

uine eata

constit- : concen connem

c1 $\frac{2100}{200}$

sisilockinmic anta souces 7
eEctap ist

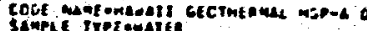

well muall Gt cTheanil rep-a

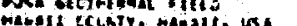

Sumping tapormat ten

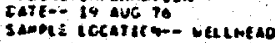

monsical oata

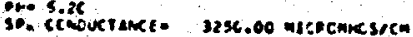

caipe ionsa

cexinit-

Censili- Clncen

S1 1000

ergitiognapmic oata
goukes

souces:-

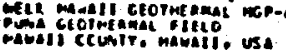

scoptins imoprayion

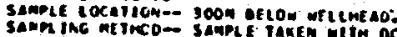

onssicm gata

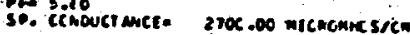

coime oara

unilyset ment

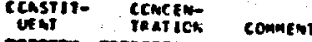

$\frac{31}{310}$

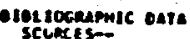

scuets

clccko ses

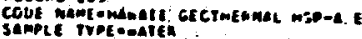




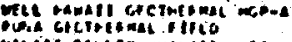

alcons ins

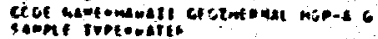

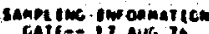

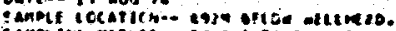

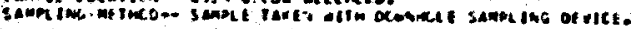
mosicm ocise

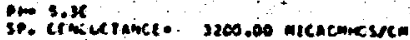

earpe oate mort

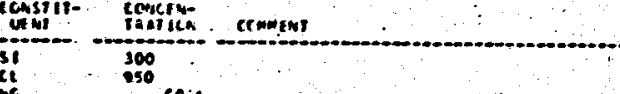

citer suspretro on saunet thi bevice.

- iocioconamic oata

soustion

secop 100. eCof mont -mawnit GeOInEmal MGe-A a

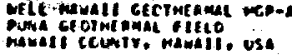

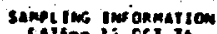

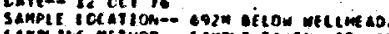

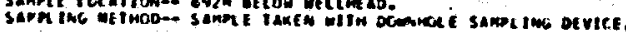

mpsicm oasin

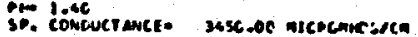

uane oula

Cons117- ERneth

esactockanic data

sourcess

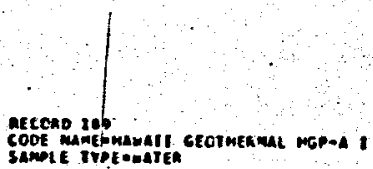

gell mamall geotikenmal igna

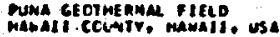

sampling informatich

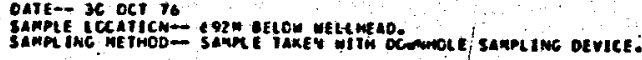

corrsicat oats

3p. Cinuverance= 3656.30 micnomosicen

came gata

Unirs-- MEN

ecmsili- concel-

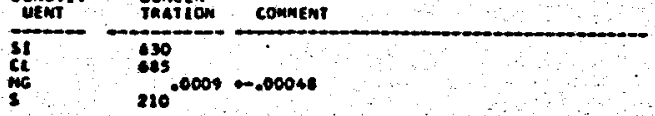

Ro: iocrapmic onta

souncts 77

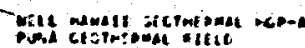

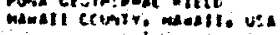

sempitus inrolnaition

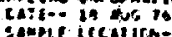

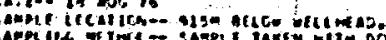

onvsical part

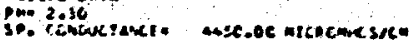

mine oale:

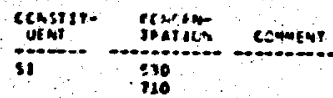

atginu amic ons

shupe ?"

accerio in

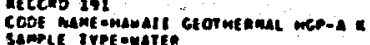

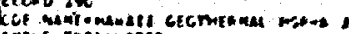

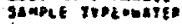

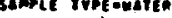

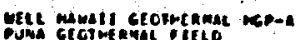

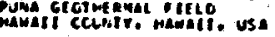

sampline smoanayion

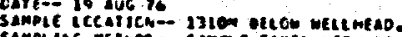

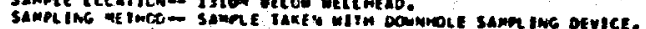

onysicat date

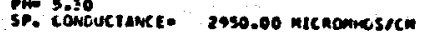

oaime outa

CCnstst- Cincem-

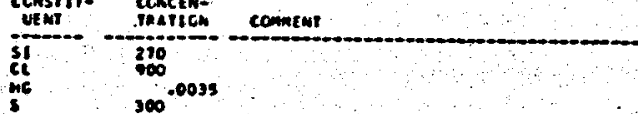

Leak susotected in sambing ofvict.

anezlograpuic oata.

sources:-

ret mavall getrutanat mor-e

aecono 102.

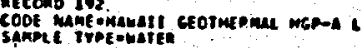

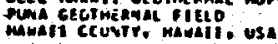

senpline ImFonpantom

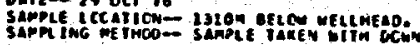

porsicat oata

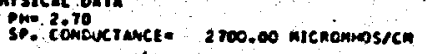

mine oATA

$\operatorname{mag}_{\text {Ma }}$

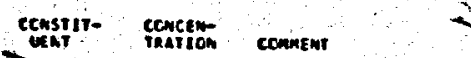

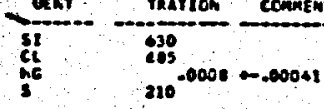

elgciognapuic oara

SCunces 7 


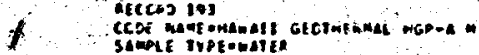

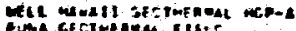

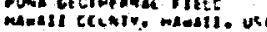

semeriting inomarion

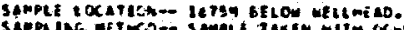

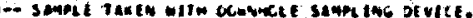

mosicar pate

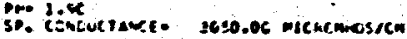

uing oala

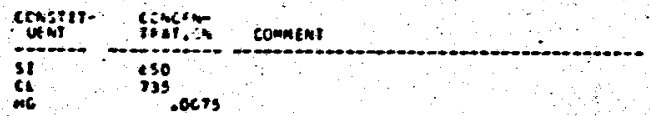

ilar sespetites in samblenc oevice.

onetrocasomic cata

scunters:-
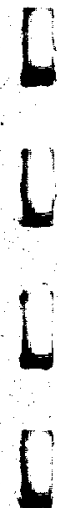

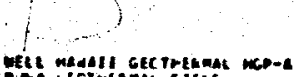

Mectes 196

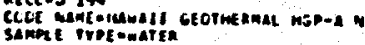

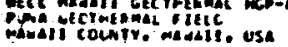

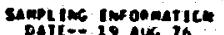

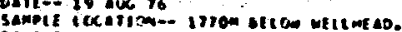

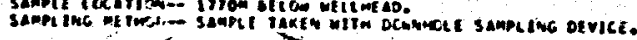

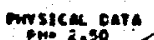

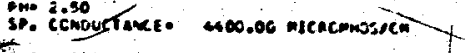

trine oats

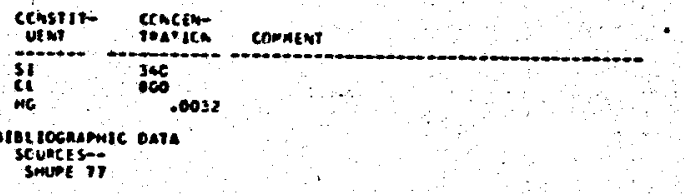

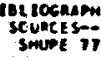

\section{OlCEAD 2 CS}

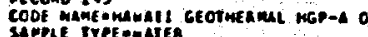

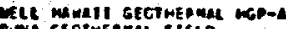

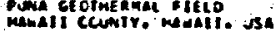

sapprine Mafokantion

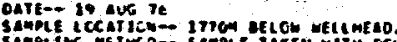

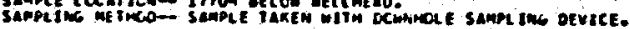

oursicat oate

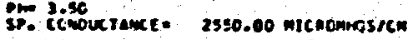

ensue oxta.

Mirs- mart

Consyir- remeen

COMnENT

ci

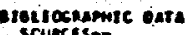

scunces $\sin$

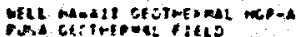

secons ise.

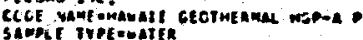

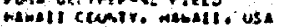

samping Im charation

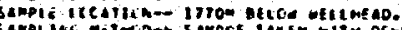

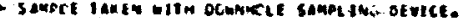

emsicas oara

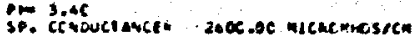

onike gaz:

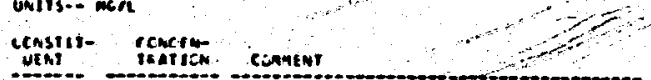

consilt- rencion

a

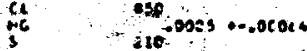

oleclochepatc oats

Sound es 17

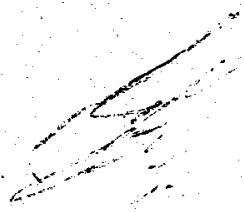

AECCOD 15T

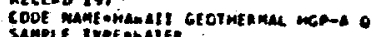

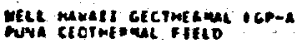

maxalt ceurty mavati. usa

simpigue enfcamanion

PAif 19.94678

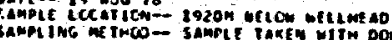

mrsica oate.

Spo. Cinouc rance. 2000.00 nicremesosoten

oriue oala

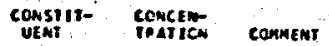

${ }_{\text {ct }}^{31}{ }_{\text {.001 }}^{100}$

- Meciochionic olta

Soukces:-

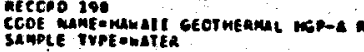

MELL Meyall GECTHERrel MGP-A

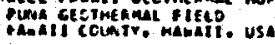

SAkpl INS ImFoannitich

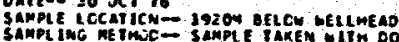

mivsical oata

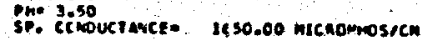

exime onth

Const11- CEMCEN-

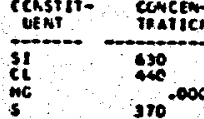

agurocasmic cata

scuects 


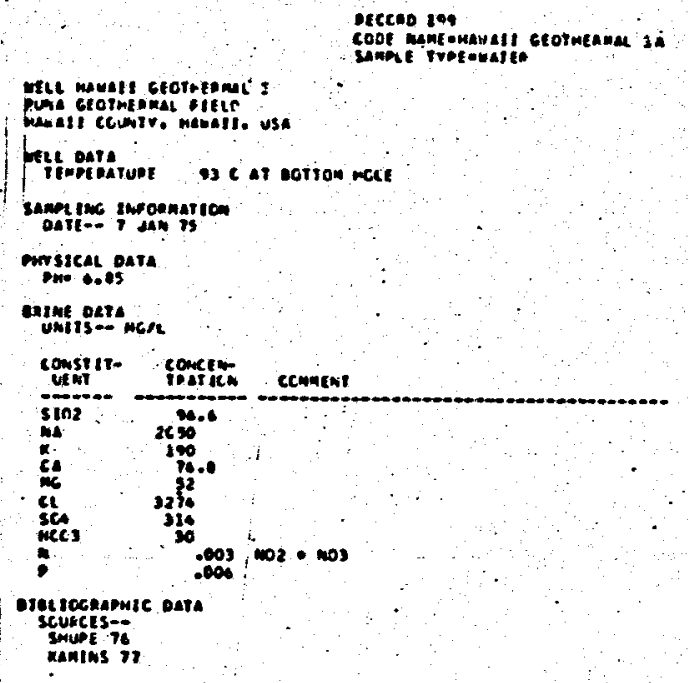

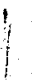

orte Matil cactorome 3

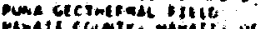

sampang imosmatice

carte- 21 ise 13

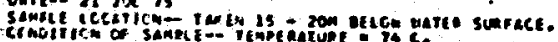

onrsien oure

cazniouts

consyit- cenceln

cinnewr

atcono 203

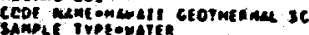

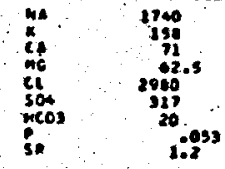

- otglockamir oața

soupt? atcero 200

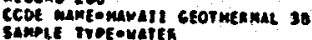

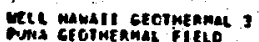

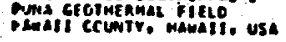

samoling imponarision

arine onst

censyly- ccancion

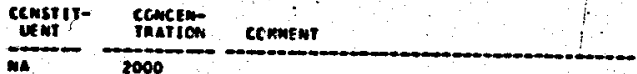

ca

$\mathrm{ca}$
$\mathrm{ck}$
$\mathrm{ck}$

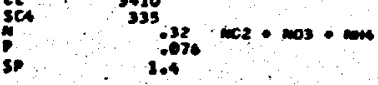

etgliochapmic eata

smope io 


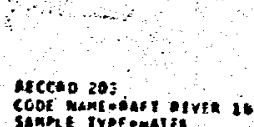

Hit pase

iccarien- niss. 201. set 33, 3

GeOF Mang

wefe is onenstion

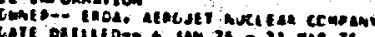

mearia

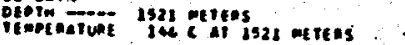

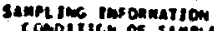

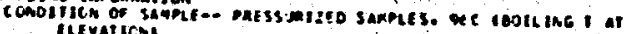

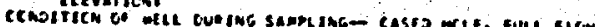

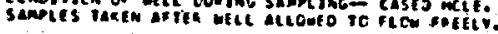

purstest oita

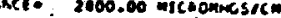

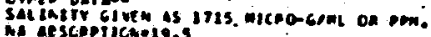

saine osta

unirs-ond units ocra cases- mear at sto

Censitr- ancen- antich

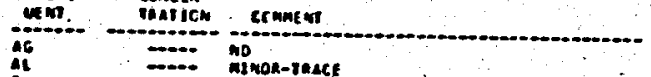

at

ci

co

Cy

(and

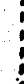

sin

in

in

$$
=
$$

mo

ingos-rence

$+00^{\circ .1}$

$\frac{3.1}{1.08}$

$\frac{4.014}{1.44}$

nace

ב.:-

$=$

rouce

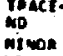

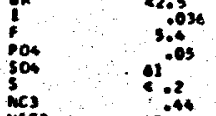

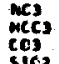

serouis

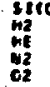

of

CC2

rork ass ar swe - 26.7 merl.

enel lockapuic onta

sounces? is
65

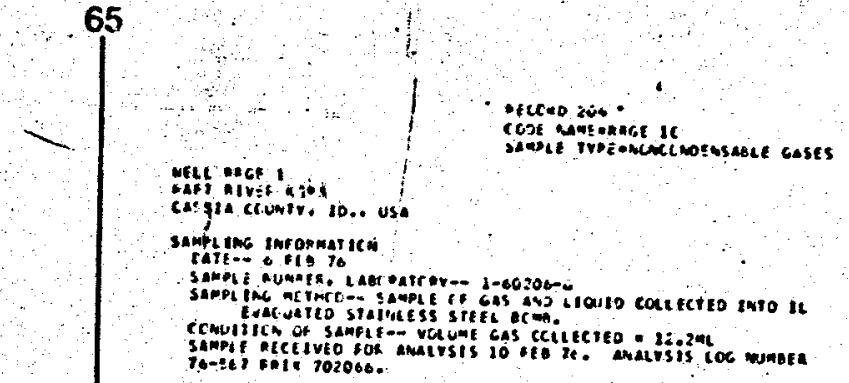

menime pata

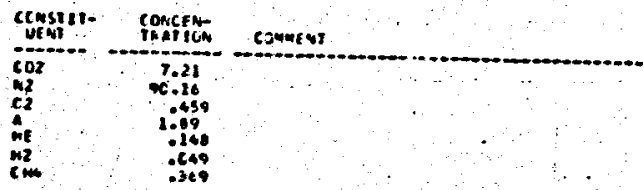

elgutrocanomic onte

matef in

(anle")

Iit aryes ${ }^{2}$

Cassiá ccimsty. 10.. usa

Sampling gmsonation

SAKPLE NUMBSF. LABC CATC RV - 1-60206-8

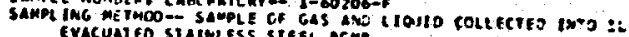

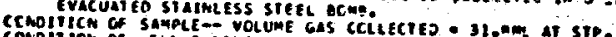

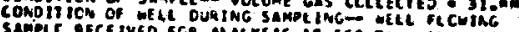

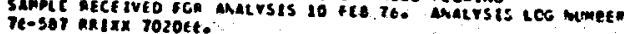

enime oate

Units ror cases-- vClune reacent

CCASTI1- CrMCEN-

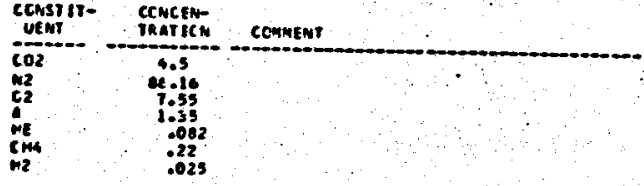

SAMPLE MOSSTELY CONIAMIMATED or AlR.

oroplocinaptic dana

scroctsist 
Alt :BGF'

\section{$f$ \\ olcono ios

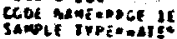

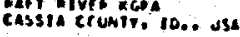

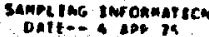

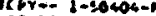

3. enecrstsices numste

emrsicat oats

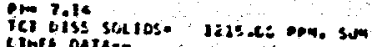

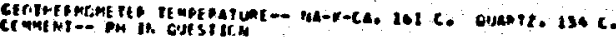

enjue oare

consill- coneris

uthi vatitio

sit.

it

It 353.0

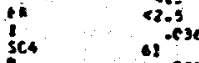

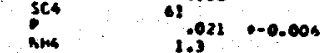

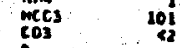

if ${ }_{31}^{2.2}$

5 i.

souktes-in

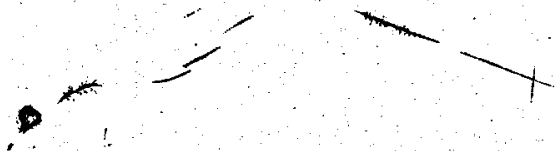

accoko 207

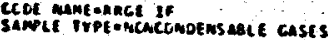

Meli nage i

Cass in ccuntr. 10.. use

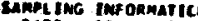

DATE-- Z3 MAR is

SARPLE RUMAEP, LACCRATORY-- 1-s0323-C

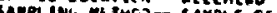

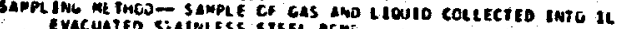

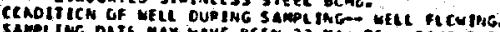

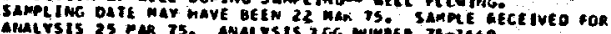

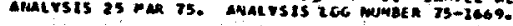

Mine Data

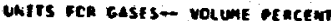

consite- Cencen-

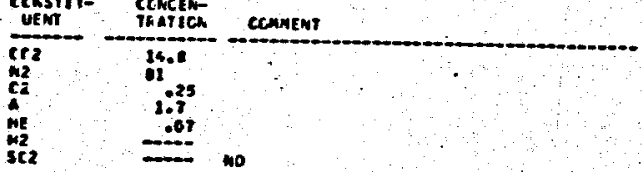

melocenphic onte

SCUACES-
Hitt stixting

chot hawe en'st to

cessia couniti. to., use

sempling the cemalien

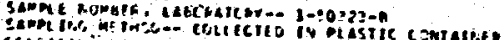

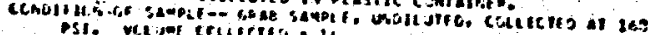

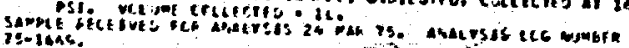

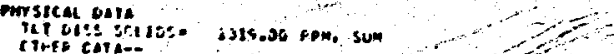

CHeEt tara

Cuine Dats.

\section{censity-}

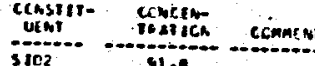

:

si.e

en

et it

S14 $\because 3^{.035}$

and 403

$\operatorname{lics}_{\text {ind }}^{\operatorname{lind}}$

IE

st

erezrograpnic bate

maite in exccino 200

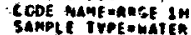

wets ato 1

CAssis clumirn. 10.. usa

Samiging tow Opwaylicm

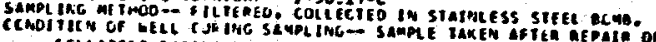
cricepser casins.

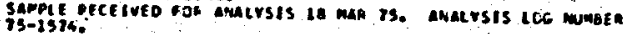

misteat on

IOT oIss sou 10s- 10iz.00 opd, sum

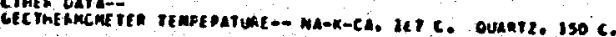

ealue oara

consit1- cchcen

Uemi Cancen-

:

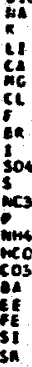

eteliograenic cat

SOUTEE 


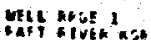

tessin cetintro. 10.. use

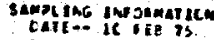

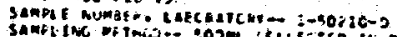

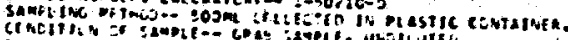

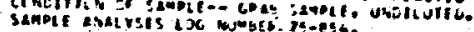

misical any

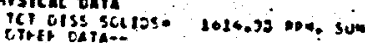

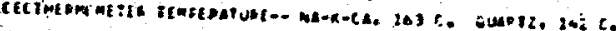

enine gare

- iselichonapric dasa

scukers-it

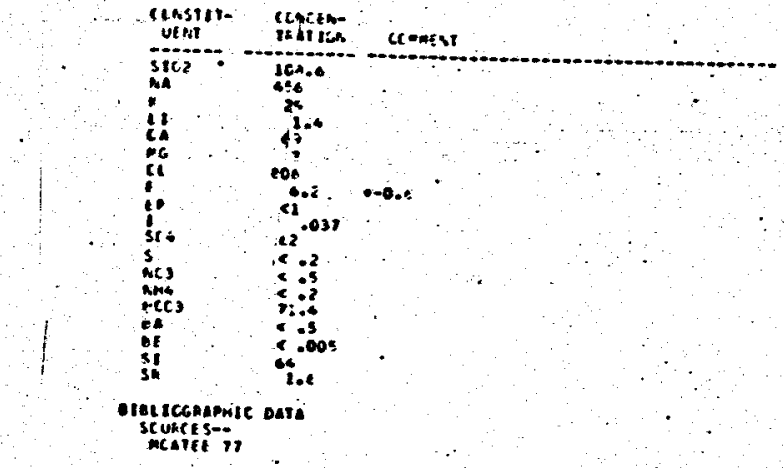

$\arccos 210$

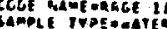

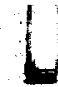

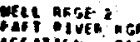

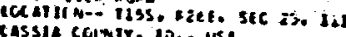

Cassir chinst, io... usa

trie jefrematica

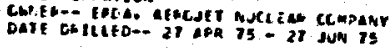

utic oenta

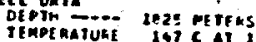

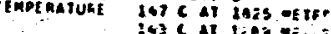

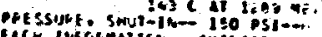

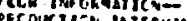

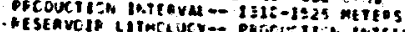

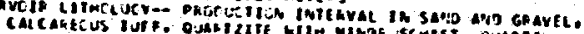

COMAE WCAICVIIE

sakpliak inzonatich

ompstch part

ORtME DAAA

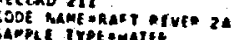

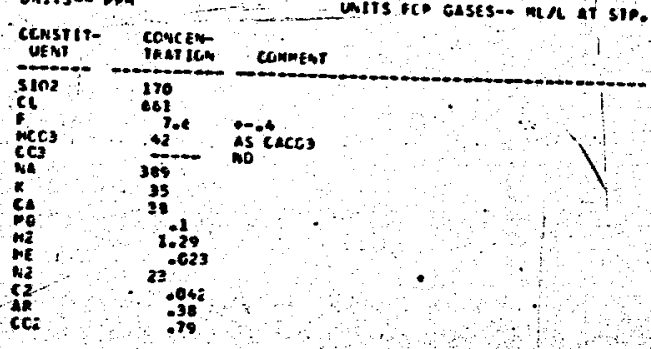

mistsographic tata

SOURCES 750
MELt toif 2

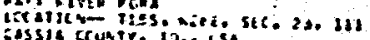

celi imormazican

nazat gave

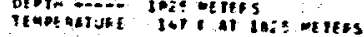

- sampitimg jassomatican

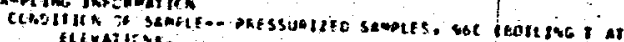

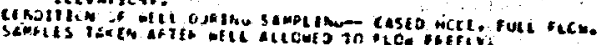

sursicat cape

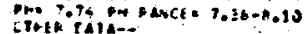

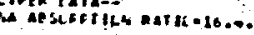

cajok tatio

consist- conoter

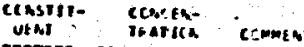

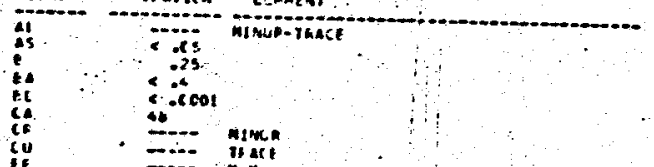

if $360^{3}$ no

it

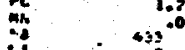

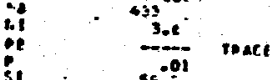

sil 250.01

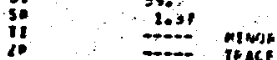

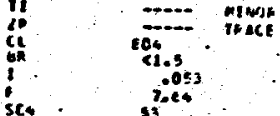

set.

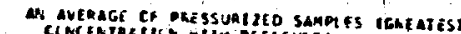

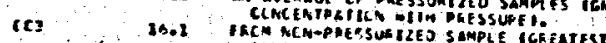

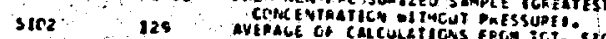

Silcowis $25: 03$

- preciccraphic oata

NuARE is
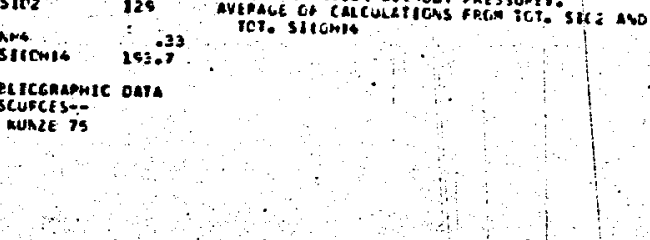

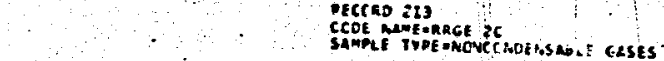

WELL REGE 2 .

senta cevisir. 10.. use

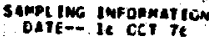

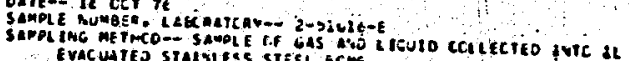

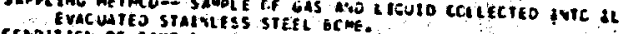

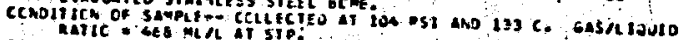

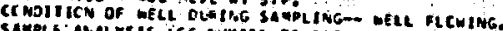

SRIME DaTA

Units rie Gaseg- Vistume tercent

consi11- croctu-

Uent IFAries Cenvent

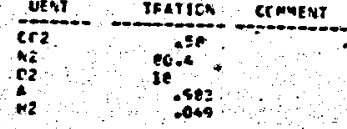

electocanpmac oate

scufects-is 
Helt ance 2 t

Cassia ctmitre io.. use

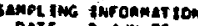

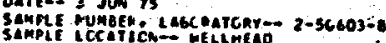

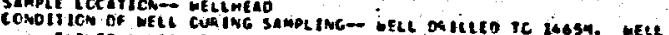

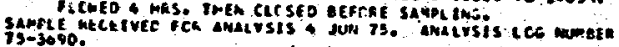

omsical oart

ict oiss sccios. 15es.or rom. sum

CTHER DAn

exine onja.

Ceastit- concen-

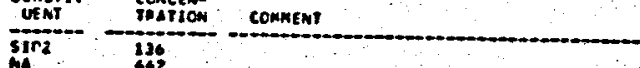

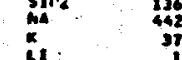

at

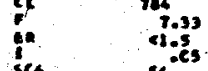

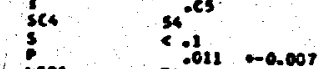

$\int_{103}^{0.023}-0$

if $: 3$

st

1 oseclocramic onta

Sounces?

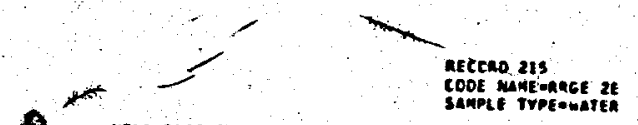

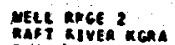

Cassin ceurstr. $10 .$. use

songting insormaticm

sape Dut Ps

Sanfing ing Inoo

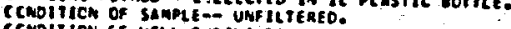

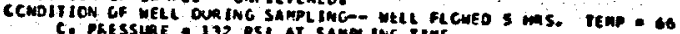

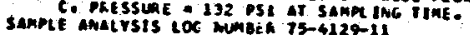

mrsicat DATA

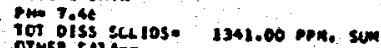

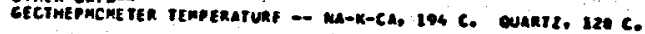

colime pata.

Conssir- cencelo-

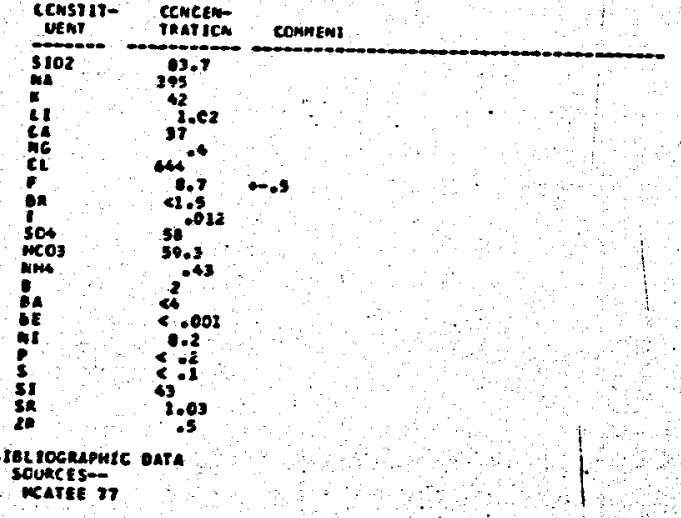

MHit motez 2

cossia recuivio 10:c use

sanplimi imponatico

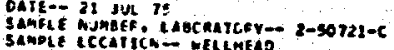

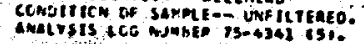

mersical onsa

ctrition.

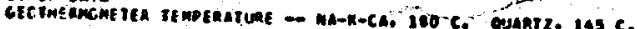

orine onta

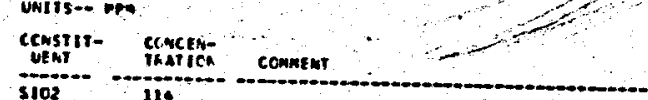

${ }_{11}^{3102} 397$

if 37

il $\quad 40.12 \quad \ldots .13$

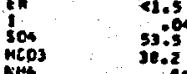

ixt.

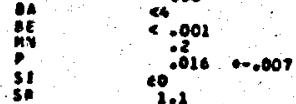

metrocranmic onta

meate in

aetcoso 210

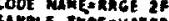

ascono 311

SAMPLE TYPE

QEeL RAGE 2

Cassis crimir. 10... uss

samprive im onmation

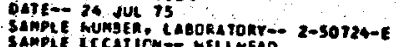

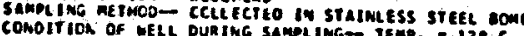

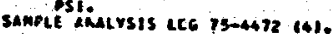

orissicar oatia

specific Gandive .

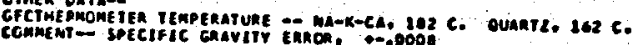

artige ouse. on

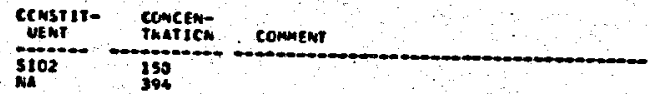

it 34

if 30

$c^{n G} \quad 752^{-2} 10.5$

$\underset{504}{5003}+\frac{38.01}{44.6}$

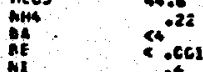

sid

ireifocraphic onta

SGURCES-" 
necono 210

CCEE MMER -RKGE $2 M$

CELt aRE 2

cassin ccunit, 10... usa

sampien insonnarioi

oct 73

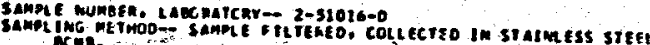

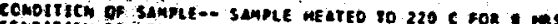

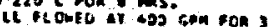

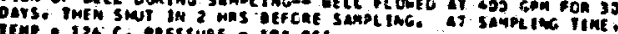

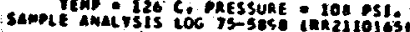

enrsical onta

OTwE o-gota-

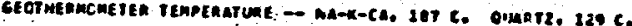

corine oata

cchstr1 - conctor

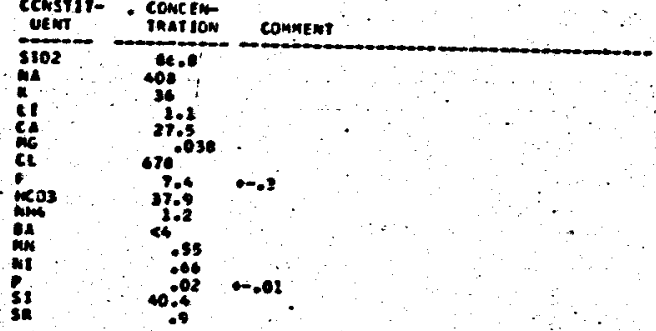

azec jogmaphic oata

watee ir econo 210

\section{COOE MAMEARGE $3 A$}

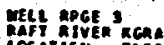

T15s, a2se, sec, 25,10

DEL Imforinarion

wicl oura

TEmónature 1009 metens

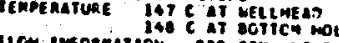

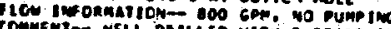

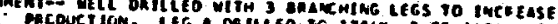

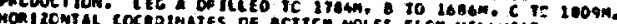

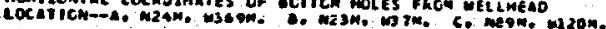

sempl tina jur oumation

Sanple cooly

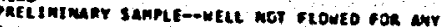

enine para

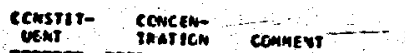

sectis

s!

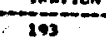

rera st

an: 293
-48

CA

Ce $\quad \operatorname{sen}_{303}^{203}$

$\operatorname{sen} 32^{-24}$

is ${ }_{6.4}^{53}$ verat -

tButiocramic exti scuices
69

MFLt PREE?

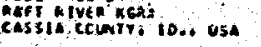

siangling in ranar tow

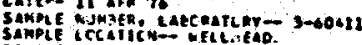

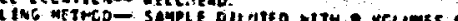

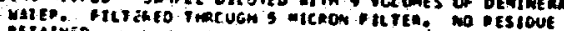

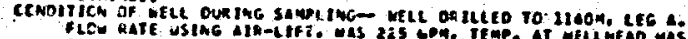

10 c.

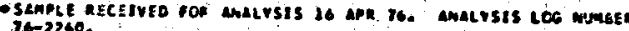

arsicat oat

iet biss sotios. sect.00 pon, sum

Carine oara.

CCWSZ1T- CONEEN-

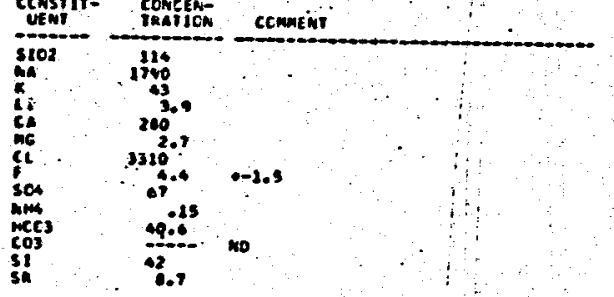

ergzocuaphic aris

SCAREE it

Heti Rige 3

Cassia countr. 10.: usi

SAMPL ING INF ORMATion

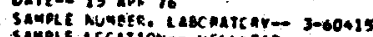

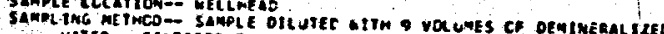

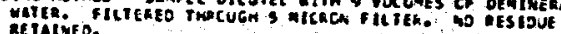

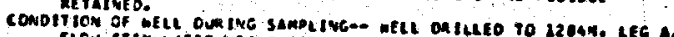

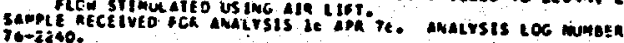

omsrcac oara

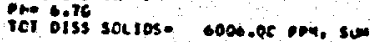

unime pare

constit- cencen

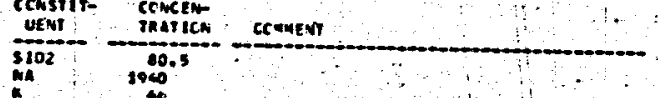

is $C$

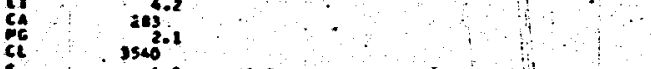

sot 4.5

$\underset{103}{\operatorname{coc}_{103}} \quad 32,5$

31 40

Ireloginentc data SCUPCES--

atcond 221

cce mamtonege 35 
Fafl aice

cassis crimitre 10., usa

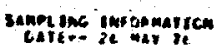

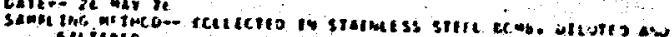

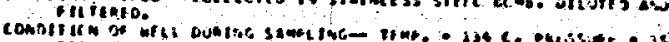

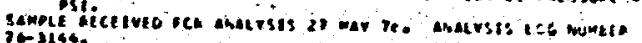

onysicat ouftanc.

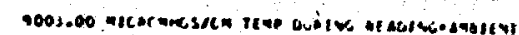
reink onta

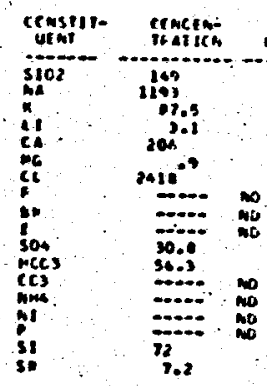

no mans neni bilicito.

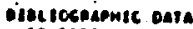

sounces:- acesc 297

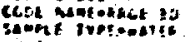
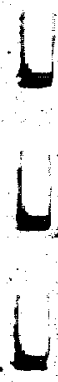

kati ance 3's

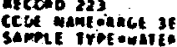

chssif comingro 10. use

SAMri ING INFognation

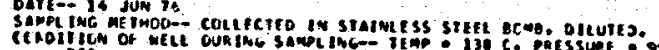

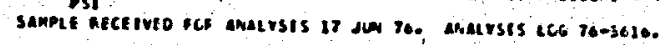

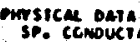

mesui oare

ccussure cencen-

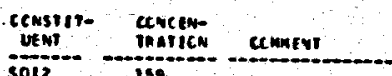

${ }_{11}^{3012} \quad 1250$

${ }_{\text {Ci }}^{C A} \quad 2330^{\circ 5}$

c $\quad=$

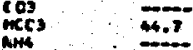

cu

it.

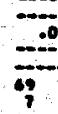

WO MEAMS MCNE DETECTEO.

-igl rographic oata

meatein

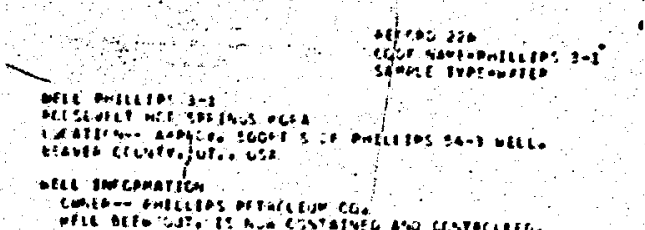

wilode

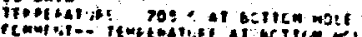

semplater ane comatien

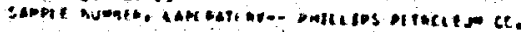

enrsical vere

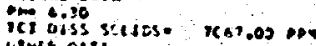

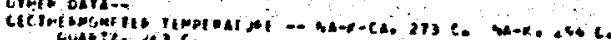

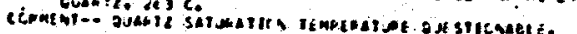

carne oare.

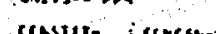

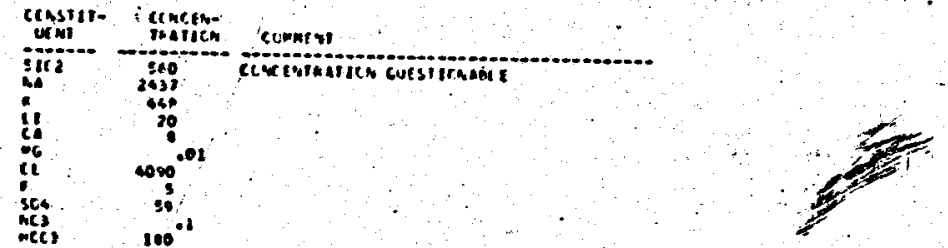

mec) $100^{\circ}$

orei rocksomic bata

srepcesso.

miteitos 18

misk too

necceo $2: 5$

CCQE AANE OPHILLOS S-I

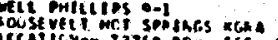

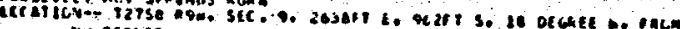
oeavie countr. vi., usa

ofle imfoamallost

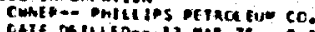

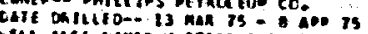

meic oats

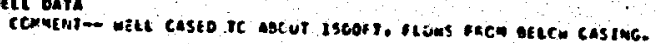

sanpr snc imroamation

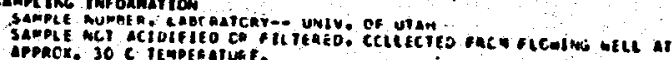

misicat oaya

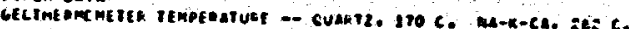

onime oArin

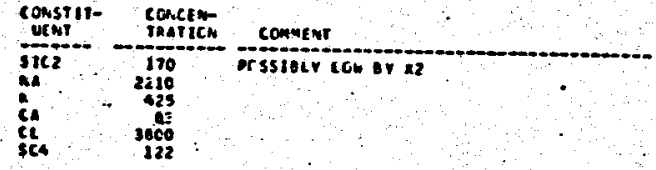

slgejocanphic oata

scuncese-

Hination

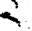




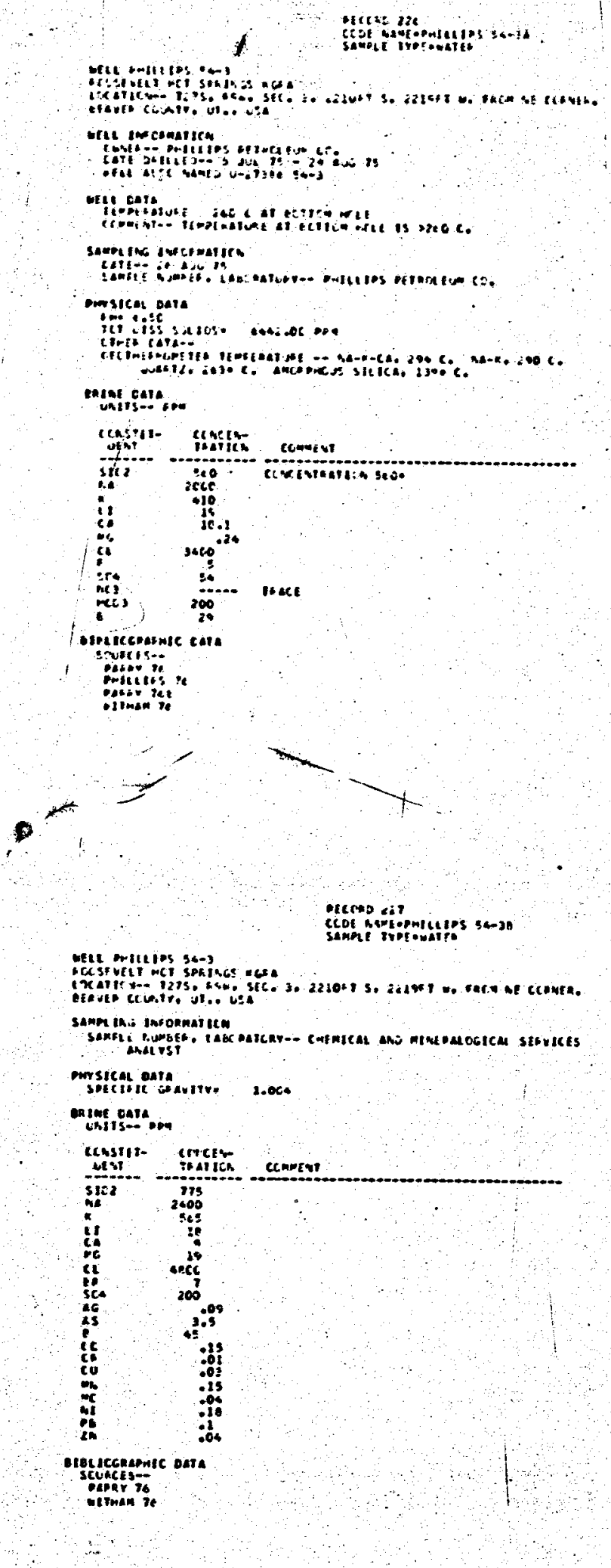

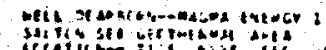

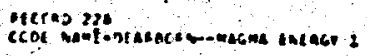

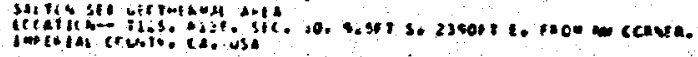

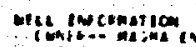

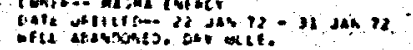

mitim mat:

elepuniopomic onta

scyactise-

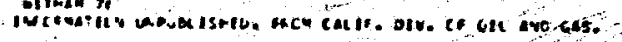

omsicer bale

icitisis

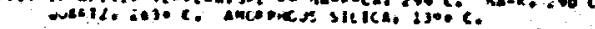

uningstosion

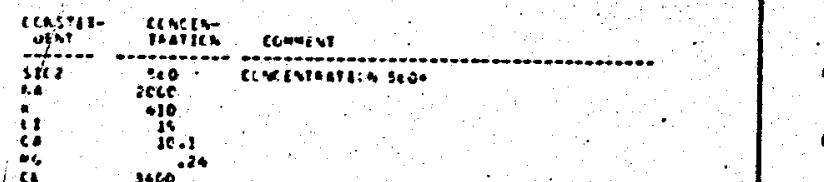

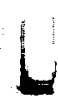

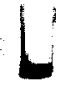

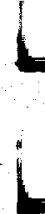

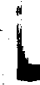

Het prlteitos so-3

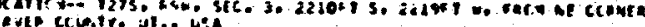

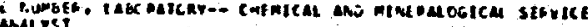

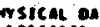

chists-e taid

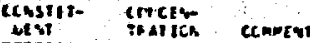

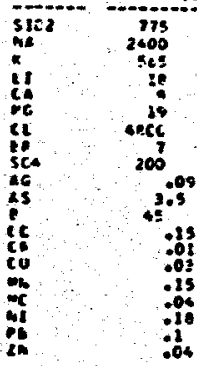

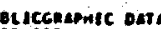

arcono 230

ctok Muntorinces:

aiccono ass

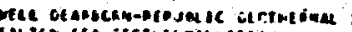

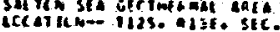

witi intomation

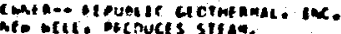

meine ouph

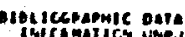

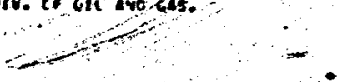

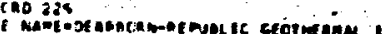

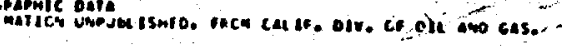

Eete tencine 1

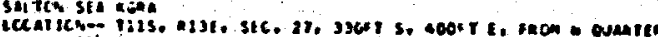

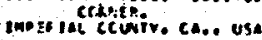

uete tim oematron

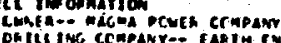

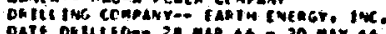

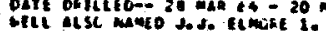

Mretoats

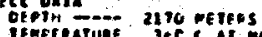

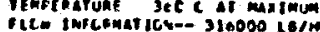

sampe inc informaticen

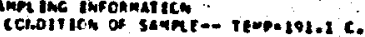

onrstcat pare

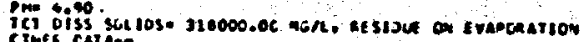

entmatorezes casica

onsuz pata

uAlls-- mon

ccaspit- cencen-

in

li. $\quad 31300$

it 201050

$\sin _{\text {ites }}$

:

in

in $\quad \begin{gathered}3500 \\ 570 \\ 1050\end{gathered}$

gats net venIrieo.

- igzichespule oata

pile

mimat $2 e$

Himan $7 e$

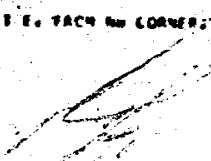




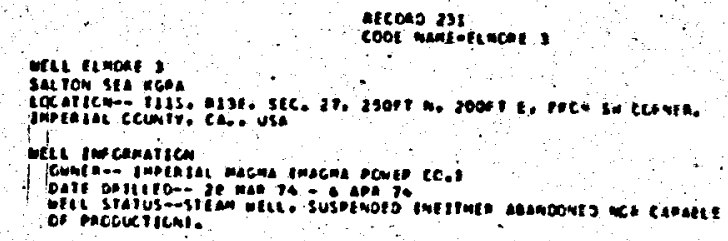

ure oura

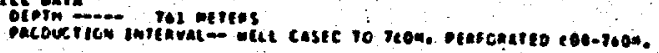

inese oate

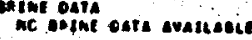

eietsogeamic outs

Hiture 70.

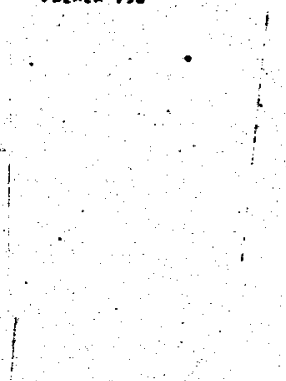

atcoso 232

cauk murfomoson is

Eet mosoin 1

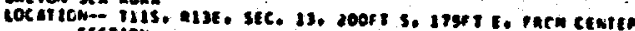

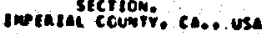

cele ingomation

cumte-- magma icuea co.

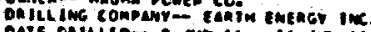

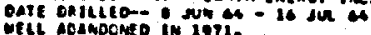

meteara

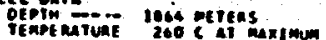

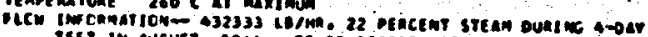

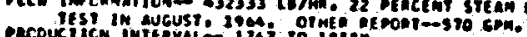

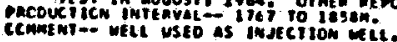

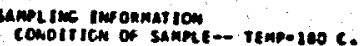

onssycal oasa

in 2.30

rime

eminaboryozis calse

unue onit

constii- coneten

UEMT COMATIL COMKENT

is

a

$-45500$

${ }_{\text {SL }} \quad 17000$

12 $200-1$

ce.

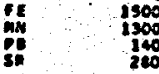

OATA MCT VERIFIED.

czaszcganutc oate

scunces-

mat 16

merping

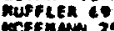

acerenam 75 niccsio 233

ccor anazemiosen 10

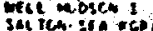

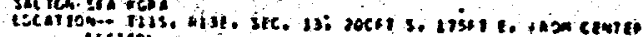

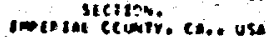

veli iromation

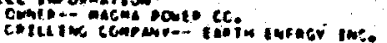

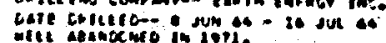

uigang

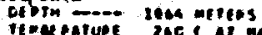

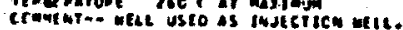

en Int oars

insisy- mojs

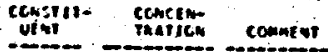

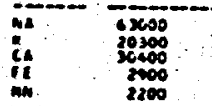

oeta nct venifteo.

- igliccrabmic oara

scuices--

merermanis

ancosthes

callot it 
vecreo 234

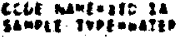

tele its $t$ ache

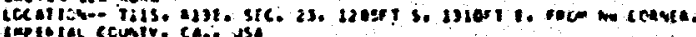

wese tercanatice

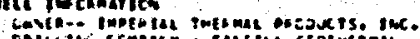

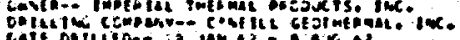

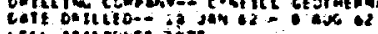

otci anaroints jorn.

witesla

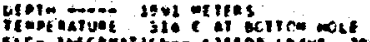

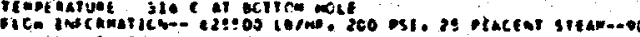

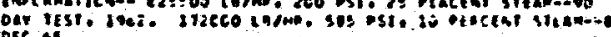

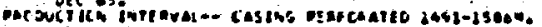

sampling imormestion

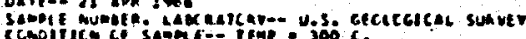

misicet oeta

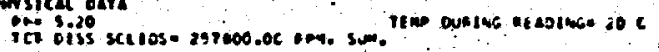

mine oara.

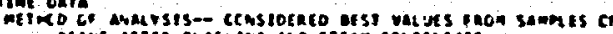

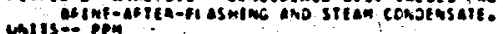

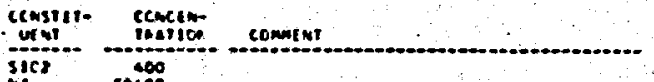

ca

it

as.

son

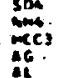

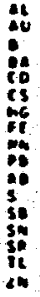

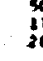

238000

15

$\therefore$

:
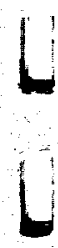

2100

SOME Acpoats Cine 2000

retac sulf ioe as wis

$$
{ }_{140}^{400}
$$

oleitreganowic cata

sowklss:-

seinuted?

ouster iso

onf 78

climen it:

iflutite 3

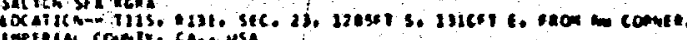

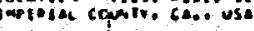

vili in conalica

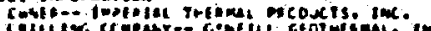

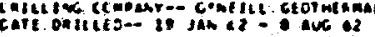

mutgen

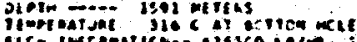

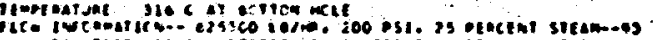

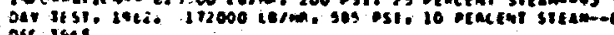

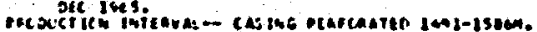

mysicas outs.

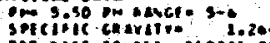

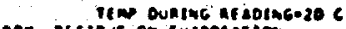

$-110000.000004 .150$

meinf ouratson

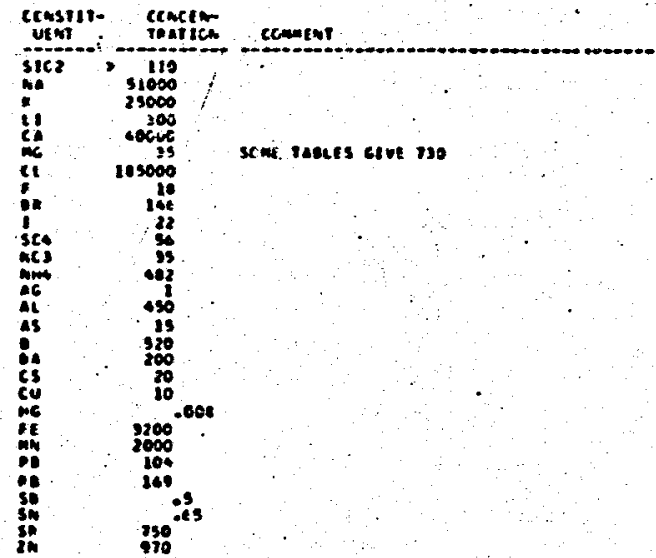

Data no conactito bon sitan loss.

eleztoganowic onta

scusctss-9

AIImER 35 
othe wo! Piveso ase

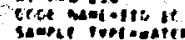

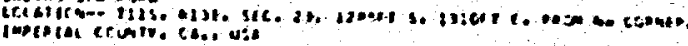

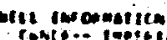

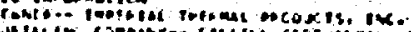

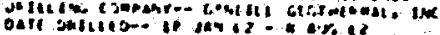

wete care

... 1991 enciss

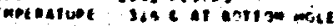

sanpigne iwsomaticn

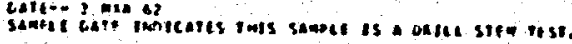

orpsical ony.

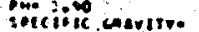

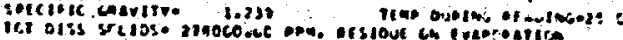

entart onle

consille cumcen-

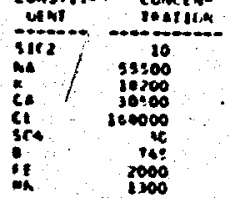

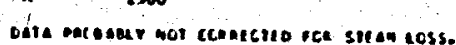

Dogiccanomic oala

scisentsion

Cemonin

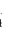
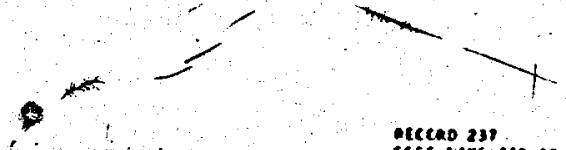

etceno 231

ceatinancillo ito

Het 1103

imptinec coumint ca.o usa

micl extecemailon

cumetr- imothial imeamal opcuscrs. ime.

mell oate

1301 merces

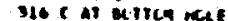

samplenc ins opmation

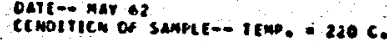

mrsicat onta

Specititi consitra

2.235.

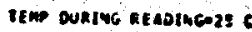

megine oars

Censisi- concin- conatery

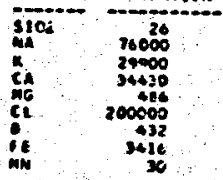

oATA monablY mot coratejeo rom stean loss.

meztognaphic oata

Sources:- mit il:?

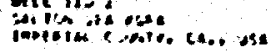

$\operatorname{cocos} 230$

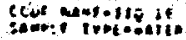

tils imsonation

whition:

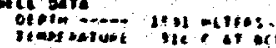

sseritis encomaticen

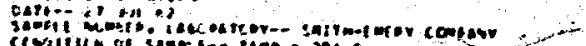

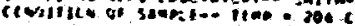

mosicm cate

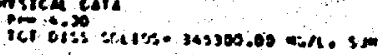

ensme ours

censist- ecrater

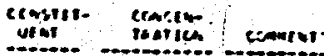

$\operatorname{sing}$

in 31090

id 36

a 12.8000

if 2905

satia nut verielfo.

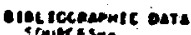

Solit is

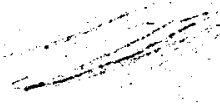

\section{0.}

eecond 239

ccok natiolio if

sat in'si ecos ca.. usa

mele encosarion

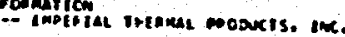

wetionte

Otorn-... 1901 petees

ictisen nout

sumptem infoamalice

mossicu oars

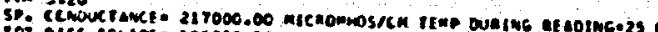

Wue oald:

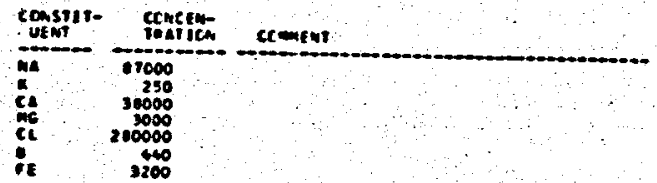

DATA nOT VEaliLE.

orectochaputc olta

$\operatorname{seccupes} 3$ 

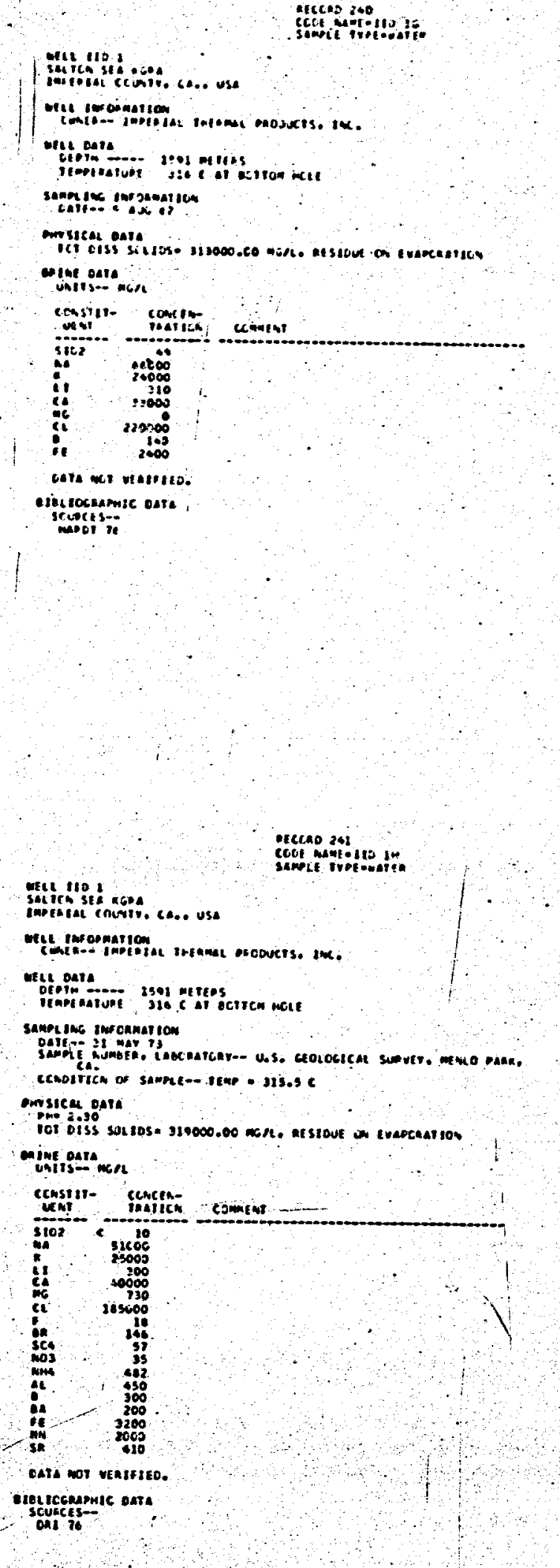

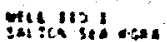

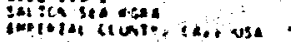

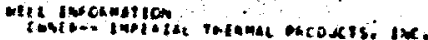

whe pere.

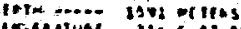

"toc cit ocition nace

sampicios promast ice

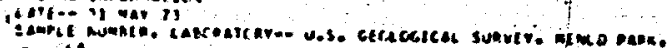

itanititin ch searece ine e sis.s c

Mine cale mols

talls-e mont

ceasili- concers

ust resters

it

unce nct venicitio.

- elsclocmaemic salá

scupess-o

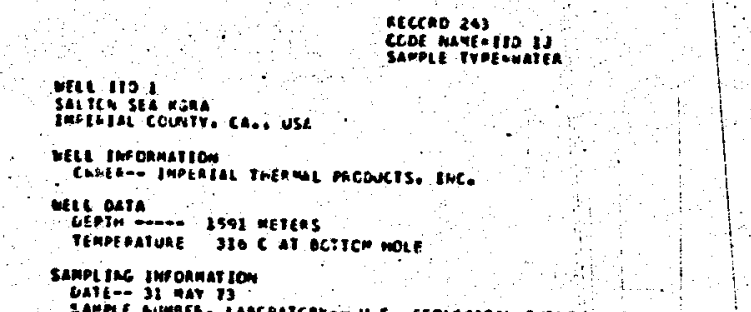

Galk- M3 WAy 13

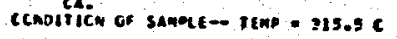

calme oatra

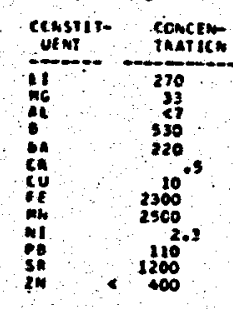

Date XCI venIfIED.

eiegiccenonic enta

scusctes- 


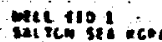

necces jos.

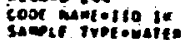

iebitilas coverve ce.. use

metringonartion

wizoars

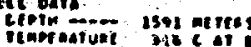

sunnaice toroonayicon

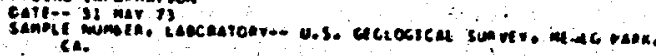

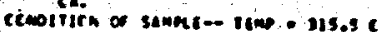

mosicas oars

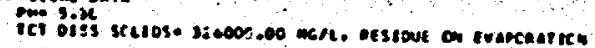

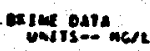

consill- cancers

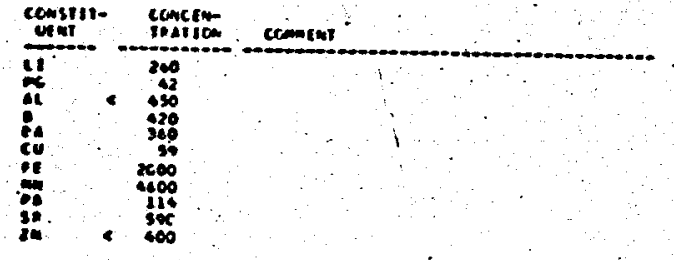

cara not veasisito.

enezrocinomic oats

scuectis-

76

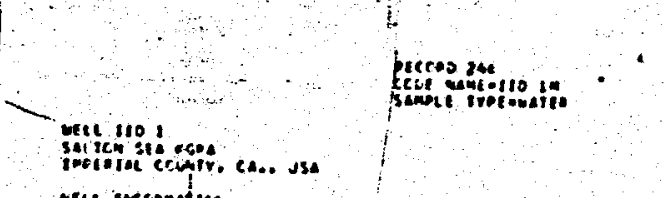

wrie incomaticm

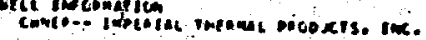

neti oull

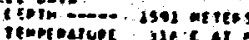

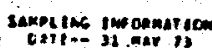

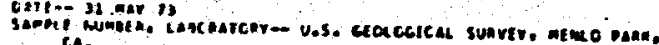

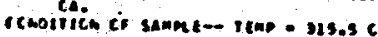

onsslect bara.

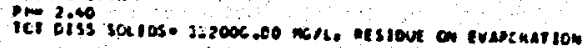

ongre date

Ccustir- cuacen

oute net bearerio.

oriol rocrapmic anta

scunces-o.

arcono 245

cook maif-iro it

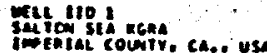

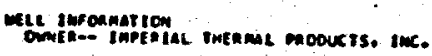

cree oura

ilprie

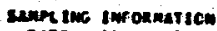

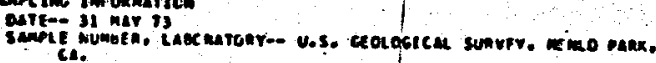

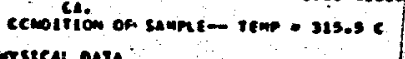

minsteas oult.

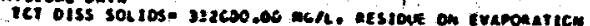

calcesene

units- Most

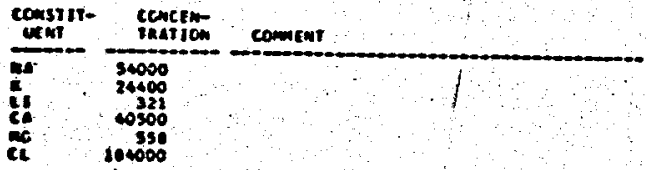

oxta not veairillo.

trgijociumic oata

sogenctse

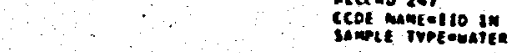

Salt ilow sít

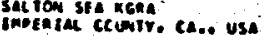

velle pesonuation

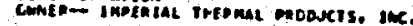

uste para

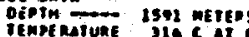

samplime imfonastica

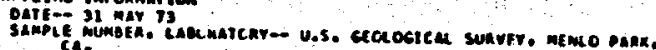

- Cenotilich of sunne-- rewp - 113.35

oursical data

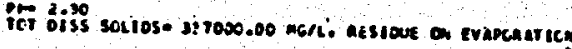

oximz oatan

censiti- cemetur

We nit imalice coment

(i) 201000

Dava nor venifleo.

cheluocenmic oata

scuncess-- 


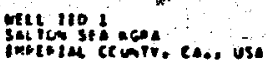

mentifeserition

mestosesta

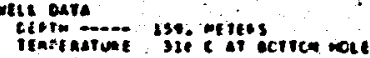

sempe juc ins canassion

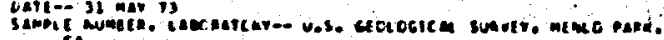

ccostifit ct senent- itmo - 319.se

morsical oate

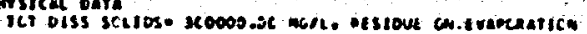

entice conta

unirs-ancen

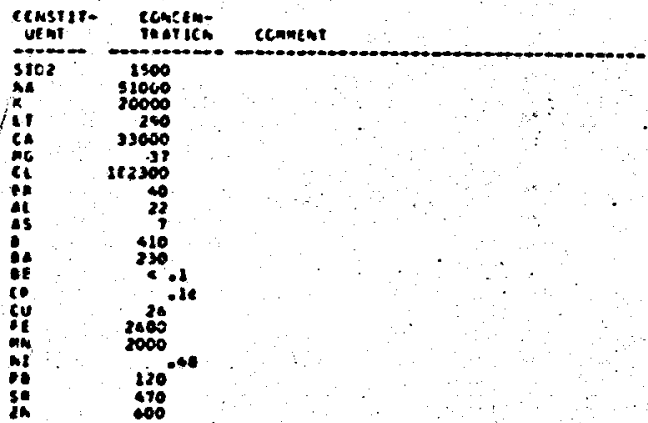

Data set reteristo.

Diblsocanoxic serte

scuks:-<smiles>C1CCC1</smiles>

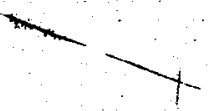

\section{atchao 241}

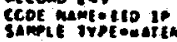

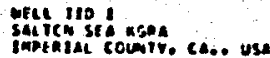

vell emronination

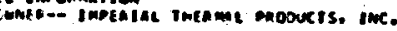

vitt oeria

sop menes

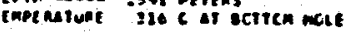

semplytic por onnayica

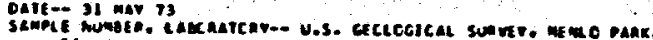
compifition of semoti-- reme - s13.s

Onrsical onja

MTME OAY

censitt- cencen-

censint Cencent

in $-\frac{11100}{34000}$

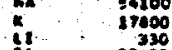

$30+05$
140
14

Sct $\begin{array}{r}263160 \\ \text { ict } \quad 2600\end{array}$

Data mor veritfito.

oreliccreanitic oars

scuptess-:

\section{coct manti-110 18}

seteis 118

saitestidisen.

mete inconarton

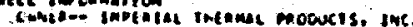

itet ony

Perme sovineteas

atert mate

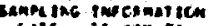

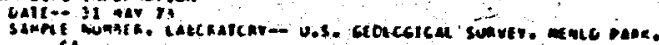

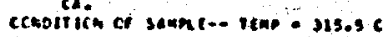

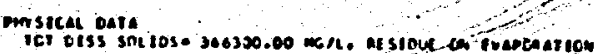

uaset pente

casti- cancits.

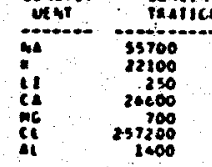

tath wor verifito.

olochoceamic oals

oni is artcod 250 comont

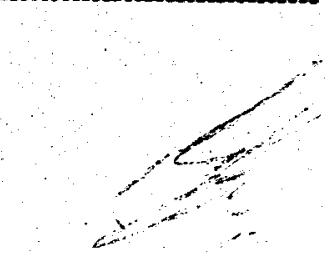

ifcoso 252

cook mantiso 18

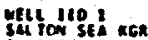

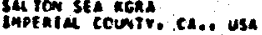

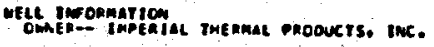

mete oare

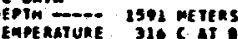

sumpresuc imforanation

at action nele

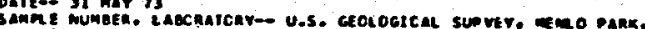

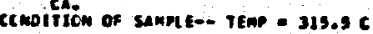

omrsicm onta

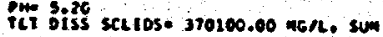

oxime outa

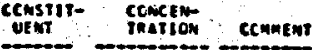

1108

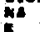

it

ci

is $\begin{gathered}250000 \\ 513.4 \\ 3073\end{gathered}$

onta nat ventrito.

moliocmapmic tata

scurc to $^{-0}$ 
accos 252

cod wenterto in

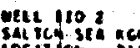

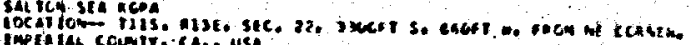

weice incentron

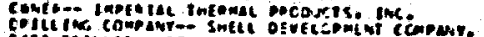
care on ilieo- 25 nor

mecl aesnochto iev?.

cric oara

1769 weres

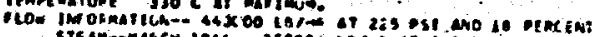

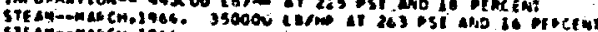

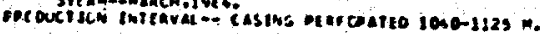

sandime intenmation

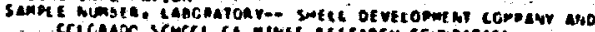

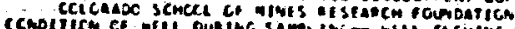

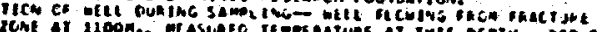

oinstical bata

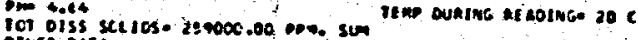

enimatorezss calce

CCNME NT -- On is cencunateo.

caine oare

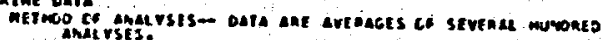

unitis- pipse

CCAsjirt concem

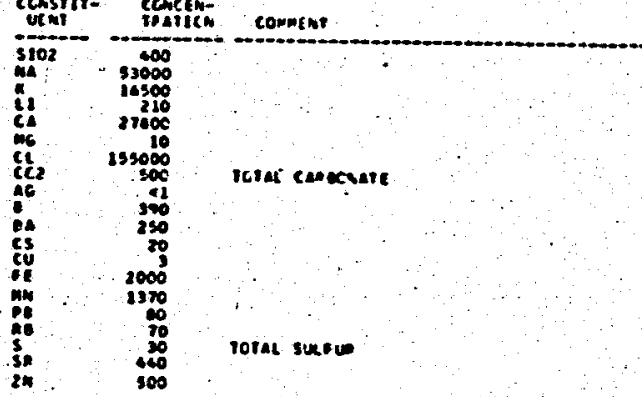

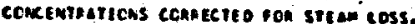

etsliocnspulc outa

sclaces-:

mitcescen of

SArinuth

mile 60

acerimani 75

cring is

ont $7 c$

Merol 1

iamoe it itechosis?

cege werctic ze

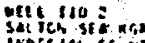

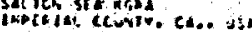

melt oars

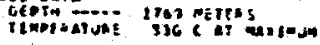

pompizes inoaksitem

seapse.. Trop - 348.0

Dersica oara

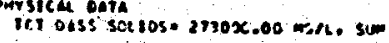

cine carte

Uw115:- MG/

conssit- ccucen-

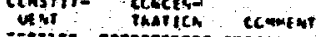

3162

s.

is

if 170

Ci $200 e^{\circ}$

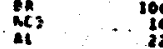

is $\quad \frac{2120}{254}$

iv $\quad \begin{aligned} & 10.00 \\ & 1320\end{aligned}$

in 30.132

Dale mot yeplitico.

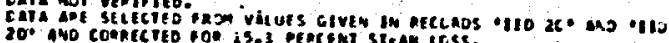
alge locpapme onta

oi ie

\section{efcrad 234}

GoDe noterilli ze

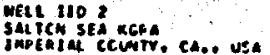

vit outa

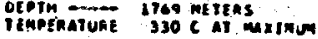

sampl the Imforinaticn

omsicat onta

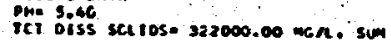

earkz ouYs.

censtom

CenstrT- CenCEN

sion

$3102 \quad 100$

is

cl. $\quad 32600$

If 100000

sits

necs

in

38 1780

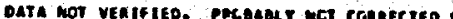

Hel HOCMapHIC OATA

sources- 


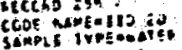

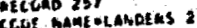

$\operatorname{men} 1132$

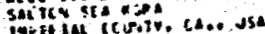

wet oay:

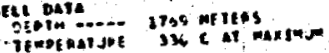

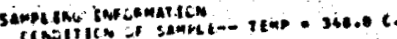

inine sata

$\operatorname{limine}_{\text {ond }}$ sate

sinistito cencts-

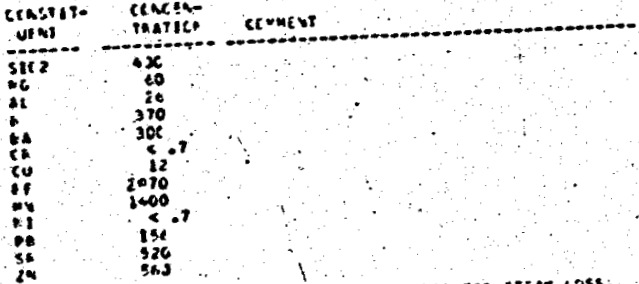

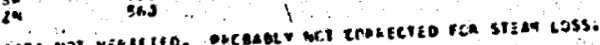

esticananice parta

twitis

inclao 250

CCOE

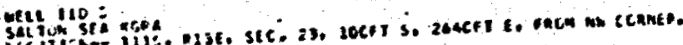

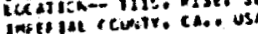

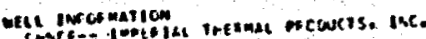

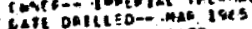

IIIC \&ANQWBO 1971.

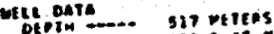

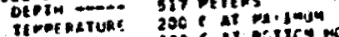

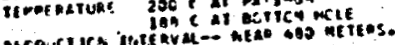

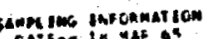

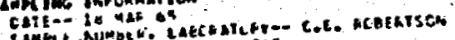

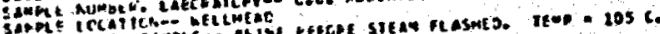

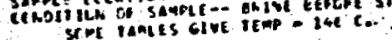

morsica eats

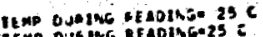

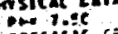
TEx:

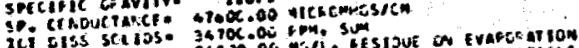

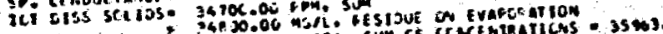

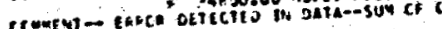

urixe tave

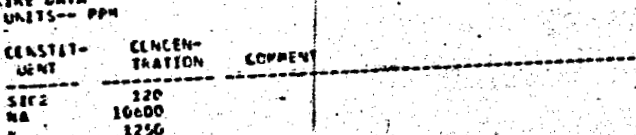

a

val ues conRtcrio erR stean coss.

- Tolicchapuic cata

scukces-

con 70

arimants

intigescin te

matos 76

PAI Mer 230

IAMDE 72

melie Laverns?

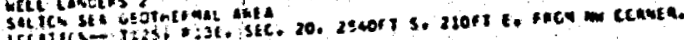
imether civitr. cia.e us

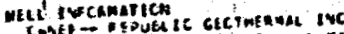

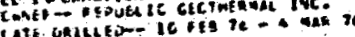

whis gath

2ane mistas

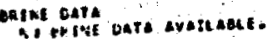

elleviccosapnic onte

3 custes 50

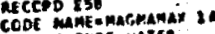

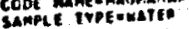

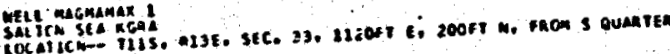

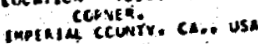

WEL INF Dimarton

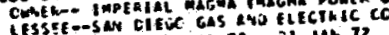

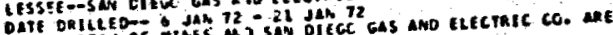

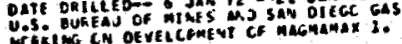

Wret QRYA a

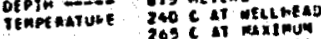

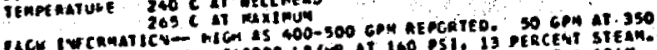

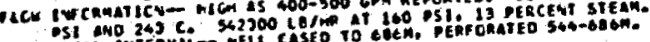

moxucticn imitavas

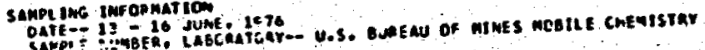

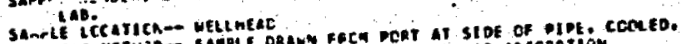

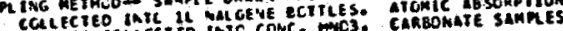

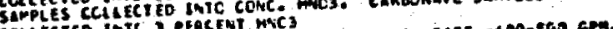
cancere

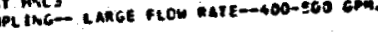

mrsical oata

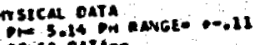

TEMP OUARE RELOTNE* AM LEM

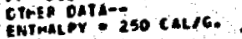

QRzNE DATA

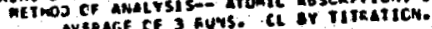

units- ory

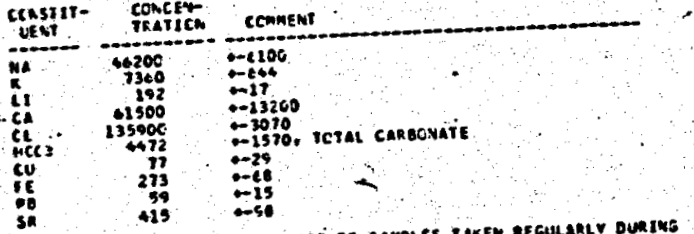

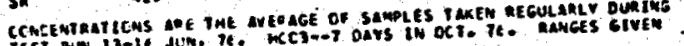

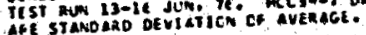

engucanomic odra

uste 108

CCIFAENM 23 .

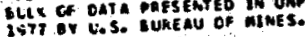


etcons 261

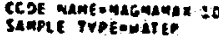

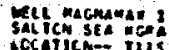

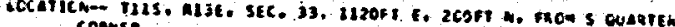

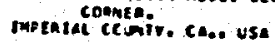

utc Imcemation

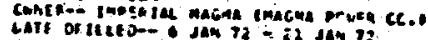

mele pata

Itrplkatute atg metites.

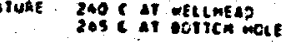

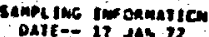

minsicai cata

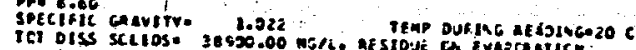

onthe oara

ccustir- ctimcen-

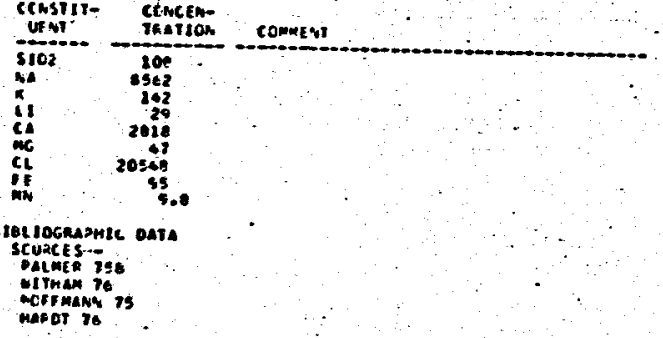

ieccors zez

teo

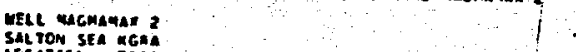

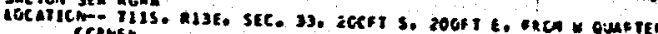
incenclal cCunity. ca.. usa

wetl theorination

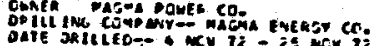

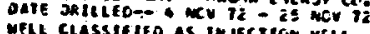

wte oara

PEETM 1329 EETERS

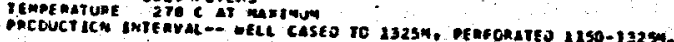

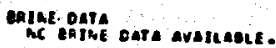

etol loginphic unte

unimen re

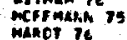

manctic it

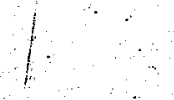

81

\section{mats}

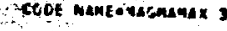

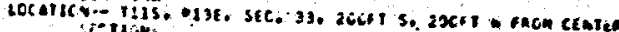

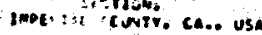

wele renkajtom

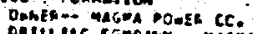

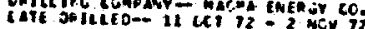

- C cenverseb to injectic 2 mer

- velt octa

OENPE

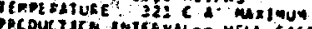

onjue on

ac ollat cata svalliable.

- Hol tocanemic onta

souces-its

Nitranis is

usw

Batrek ist

Hetl mamsunaz:

Recono zos

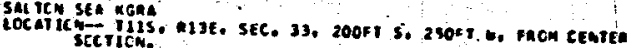

Iaperial colvtr. can usa

wete inforasison

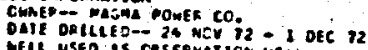

well useo es ceservation tella.

Gel aca

INo meTeks

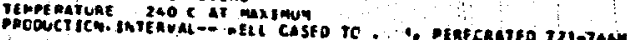

erime onse

ugrogrimic onsa

irmain io

merinani is 


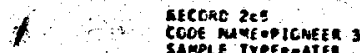

EEt romtri 3

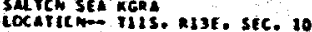

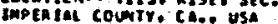

urel MaroRmation

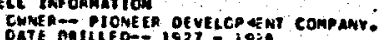

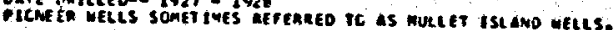

mite gave

PEPTM

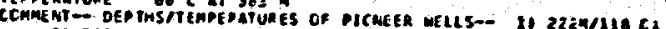

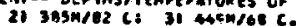

SAMPLine IMFORMAIION,

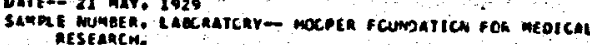

mrsical oata

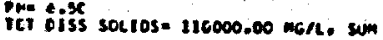

mine oara
untrs - ware

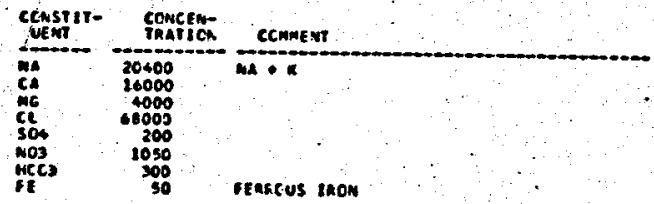

etBL iocaupuic oata

coukcts-s.

chinatinn

$\lim _{\operatorname{cosil}} \lim _{i 6}$

atcono 260

CODE MAMEGR IUER RLNCH gh

MELe River aunen 1

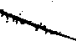

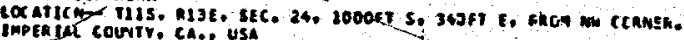

- meli imormation

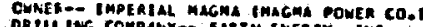

Dafe

wetl outh

OEPTH 24at Meters

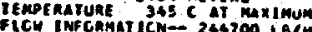

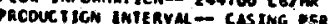

morsicas oara

The 5.20 siss sol

CMER DAT A-

mine oata

UKits- MG/4

CONSYIT- CONCEN- PATICN

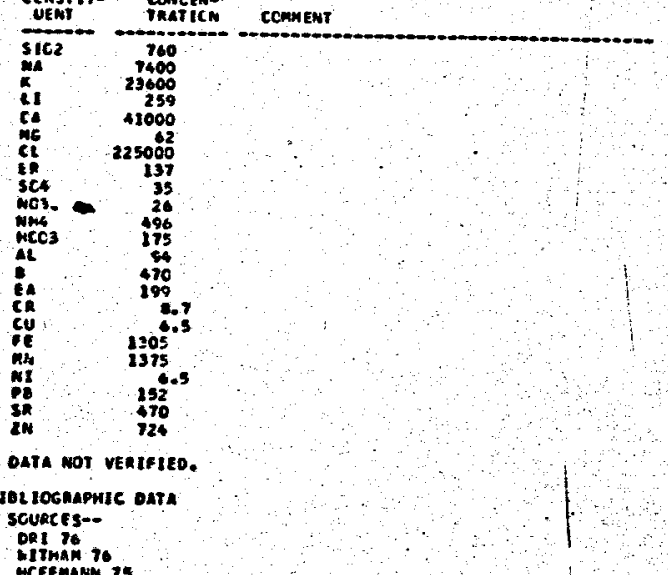

\section{accea zor \\ crog mangarivgr enen it}

meteriyes Bain

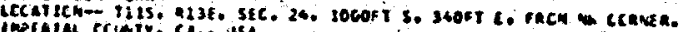

utic unfopmation

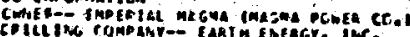

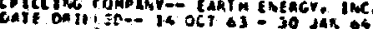

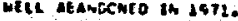

vere cars

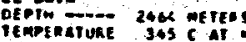

mirsical oarh

ime biocs sx

eaime pexne

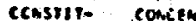

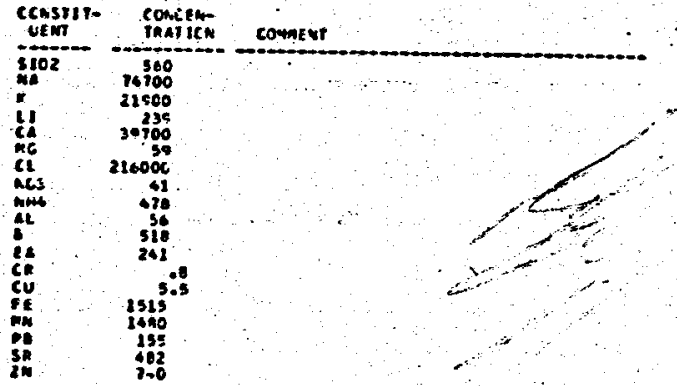

Cath mot yenirito.

oreclocreapuic eate.

scurcess-

ciman in,

AECrad 260

Mete simetate.

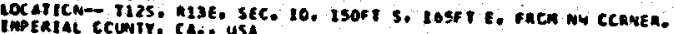

vete smformaticm

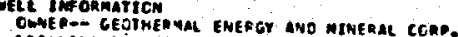

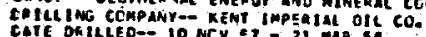

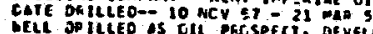

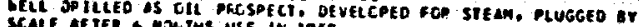

weil on

oxime oate

Degine OAI Avallabie.

ergegegaphic oata

seurcest-

itrian is?

PANDE 76 
actues 260

CCut mancestriecall :

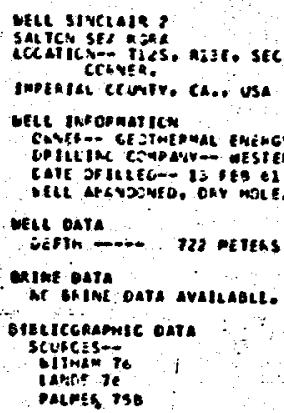

\section{$\operatorname{coc} 200$}

Cece mantesinciain 34

mete sincente

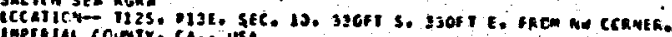
couvr. ca.e usa

wit Ineconitich

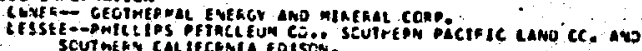

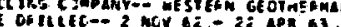

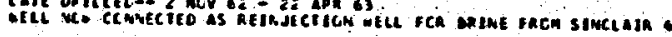
vete oave

JEOTH 231 meteks

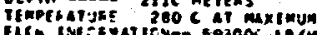

HCW INE

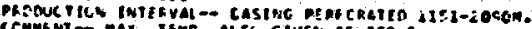

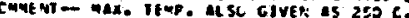

Sanbr tws Enrof mation

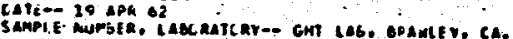

oursical bata

prosict

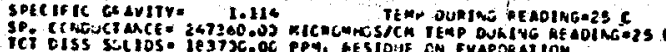
c 153300.00 opy, Sum

CThE cara-e

Wine para.

CENSIIT- CONCEN- TCMAENT

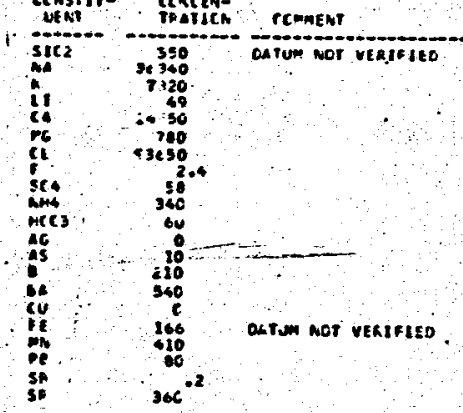

VALUES ARCBABtY AGT COKRECYE O EED STEAD LCSS. etei lographic oata

SCufCe $5-$ -

MCFMAR to

neligesr 7 to

Patmét te

cutiles es

OOE 76

MARET $P$
83

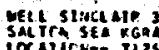

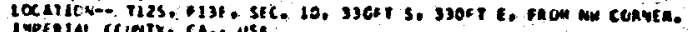
cc cintr: CA.., usa

wete ineornstion

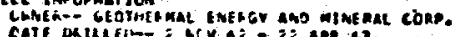

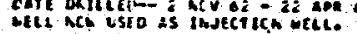

Metronta

al10 METEHS

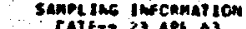

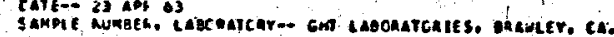
misicat oare

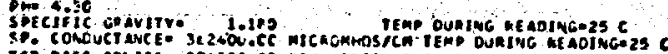

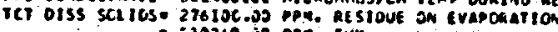

oatine carta

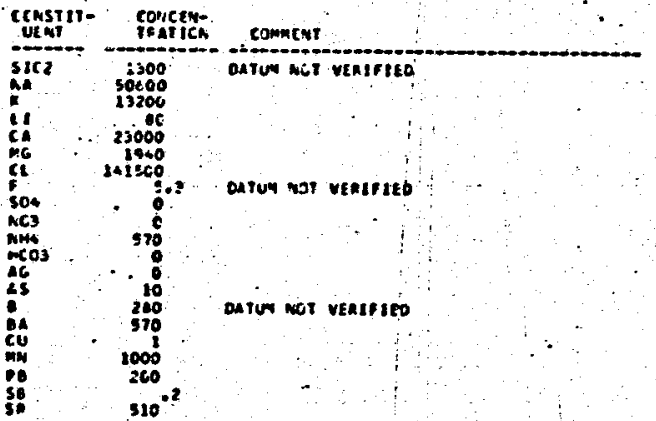

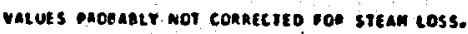

orec tograpmic oata

conk 70

of I is

AECORo 272 CCOE MAMESSINCL2IE IC

SELL Sinceain

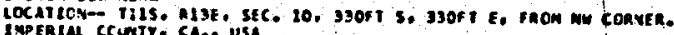

weLl enfopmajicin

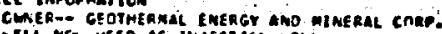

AS INJECIICA MELL.

WetL DaTA DEPIM 2110 METEAS

SAMPL THE JW ORMATICM

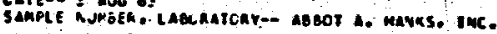

purstcis onta

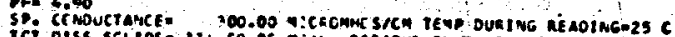

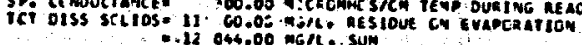

okine Data

censtir- coscen-

me 10409

${ }_{0 .}^{20306}$

CC
SC4
MHE

in ouestion
in euestion,

teclockapwic oATa

SCURCES-

Op 176

mitran te 


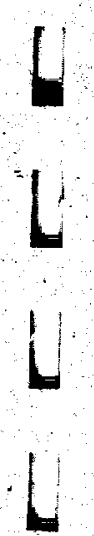

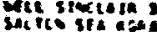

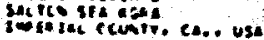

semic inconation

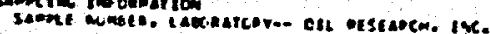

minsece gare

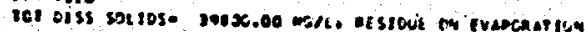

mine seiv

cessit- cenefon

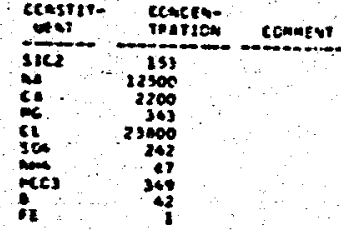

ogie wo veraterto.

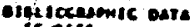

setisen

-icces an

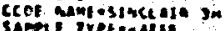
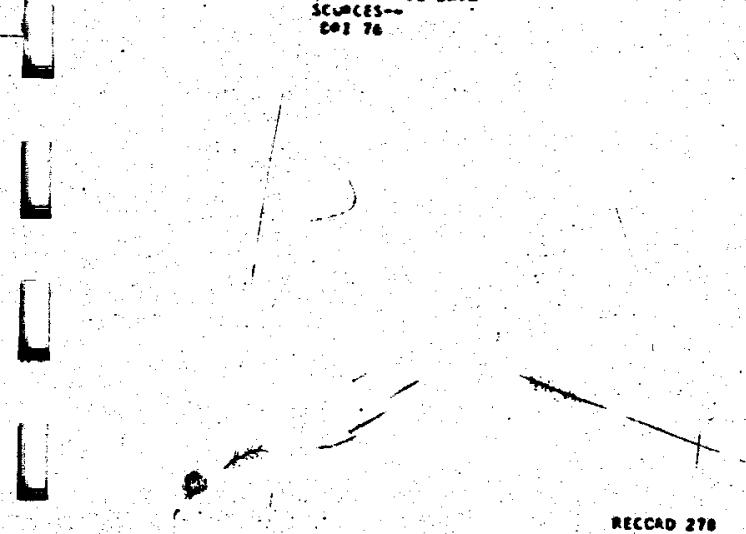

AeCCAD 270 cook mank osinciata 3

vete stwieras

iefifin ecurity. ca.e us

sempicic inoprartioes

mysicareats

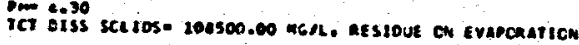

colike cara

censrit-

censti:- conctor-

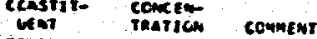

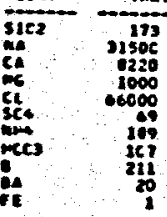

Bata ot venifjeo.

orgercerimic onta

Der 30

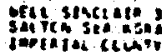

ccot uniestricento

sanglino incenarica

mosteat vare

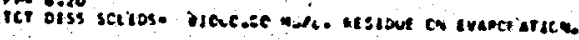

eagne onta.

UNITS MES

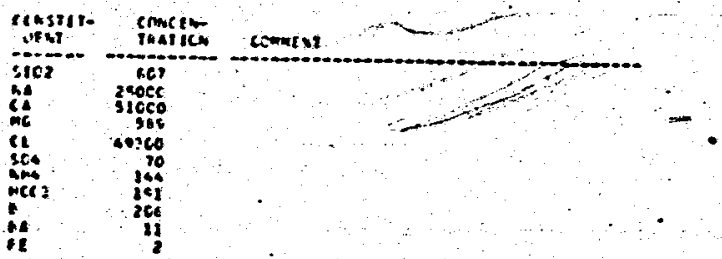

para vor venterto.

ereticgabmic oata

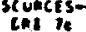

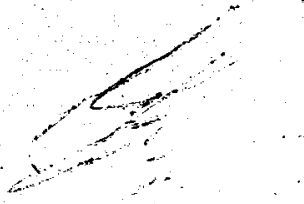

aeceno 200

CCOE nank osincLate in

Mete simerasks

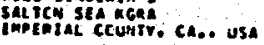

samplasac imsonmartom

政

TuY oiss sol

CunE pata

ccusstit- concen

Paetica

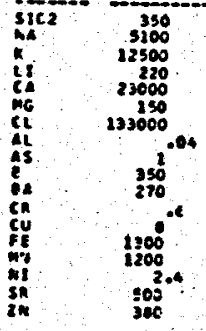

oate nat venifito.

iegrocrepnac oata

D*1 it 
notos an:

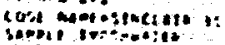

Hit swals:

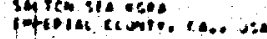

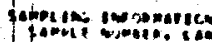

mosicu asts

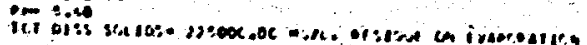

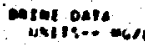

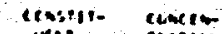

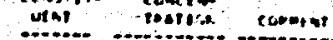

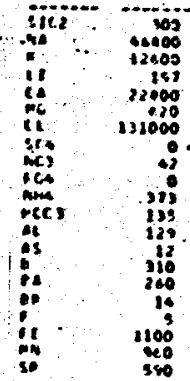

gais not vesiorio.

etectoceapric oata

secuet is is-

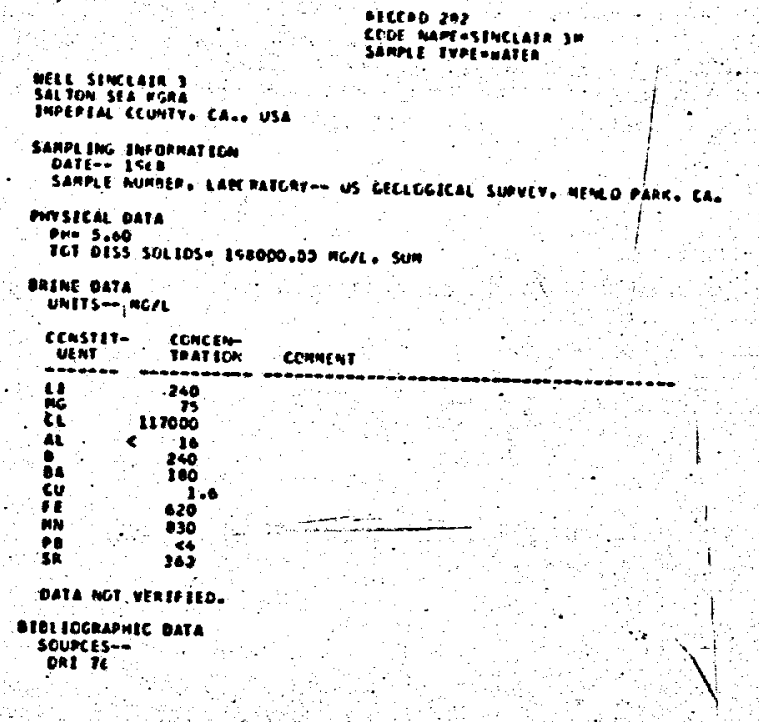

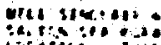

icress?

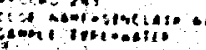

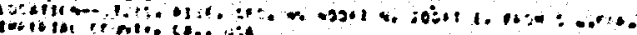

urce juchention

nets

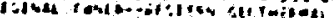

mith cat:

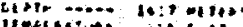

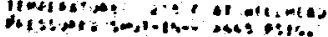

sampins insomentem

antie acot:

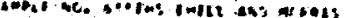

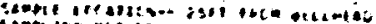

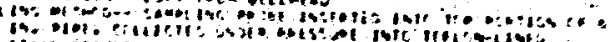

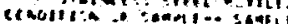

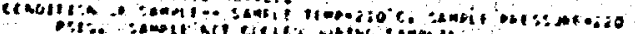
conopif

mrsecas eate

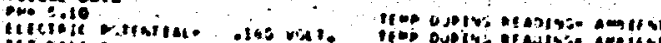

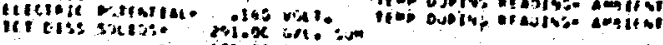

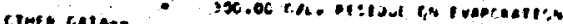

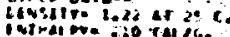

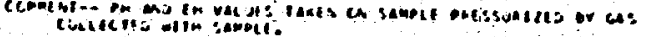

Ringe cala

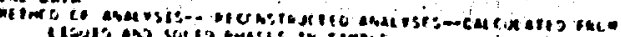

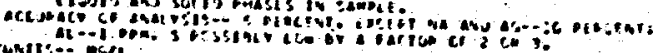

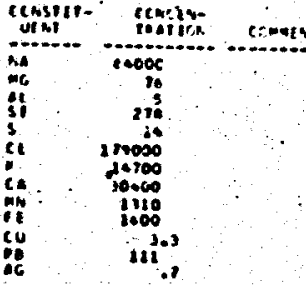

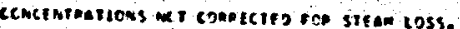
elezpociansic oale

srobets is

mimen

meraming is

murcesen..." 


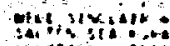

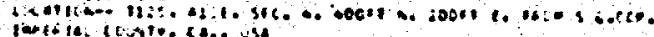

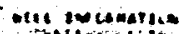

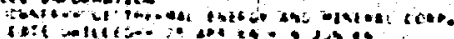

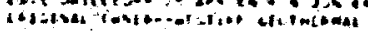

otic oen.

Non

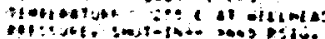

sempinc the comentste

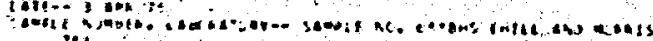

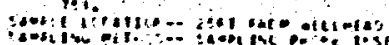

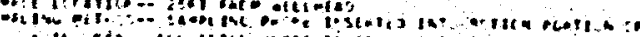

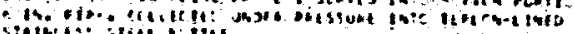
corolifo

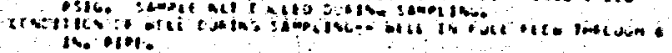
$\operatorname{mosick}(a)$

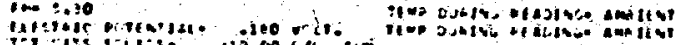

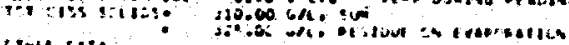

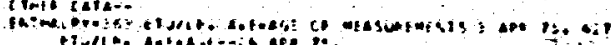

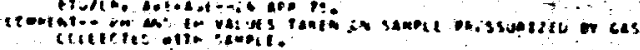

minterte

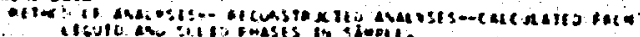

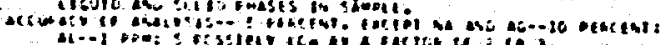

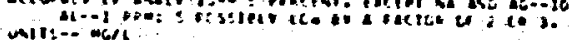

rgsise.

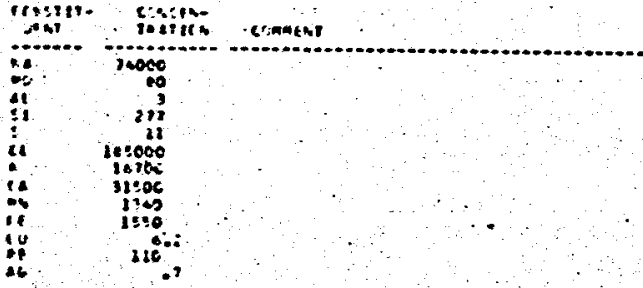

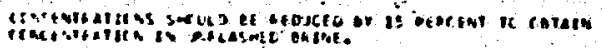

oleciocenomic Data

sestitis is

itima ie

mastinges

ats

atches

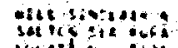

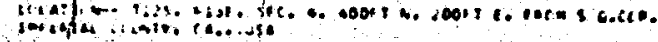

Heci inconorson

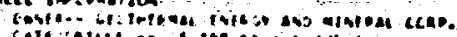

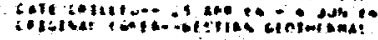

matcants

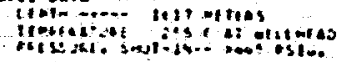

sauritat itsonatein

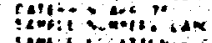

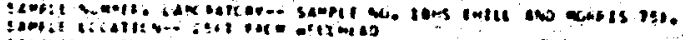

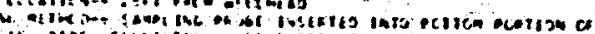

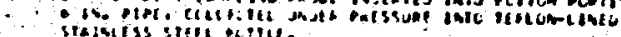

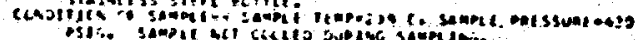

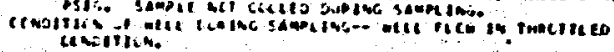

\section{onsticat sati}

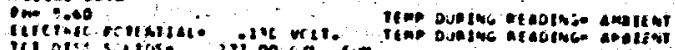

comors.

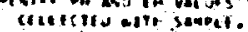

ming pare

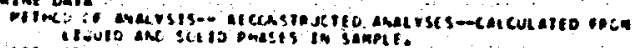

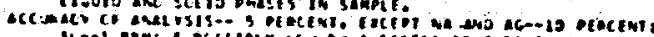

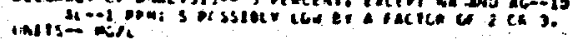

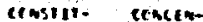

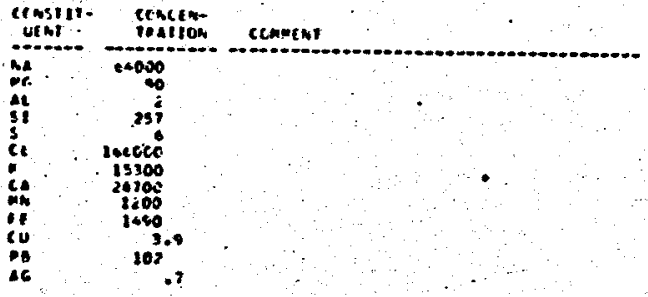

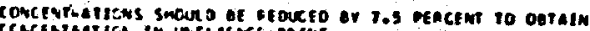

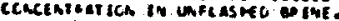

orgejecceumic oata

niti is

netsme- 30

merraches,

mackes its 


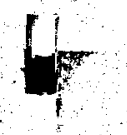

mat sum sta.t:.:

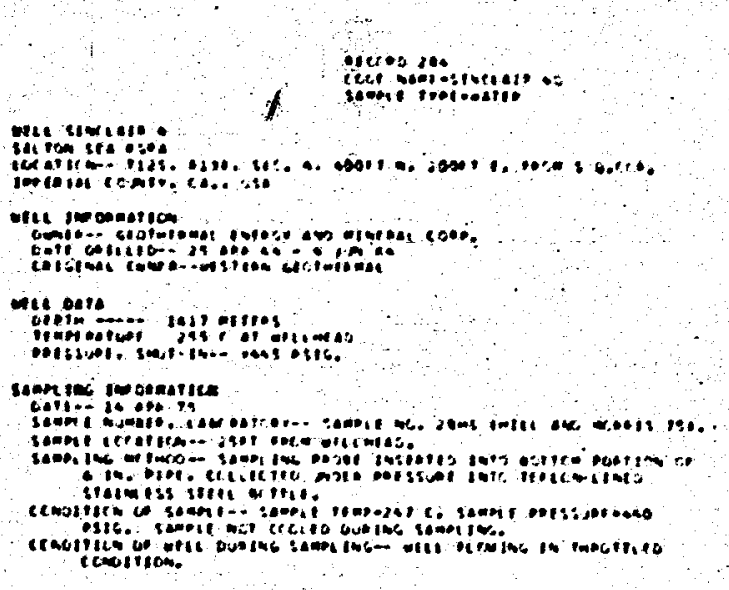

monsiea oare

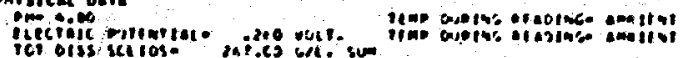

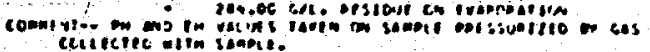

onjere pate.

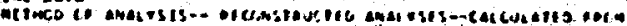

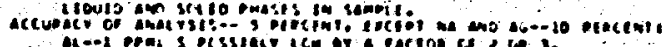

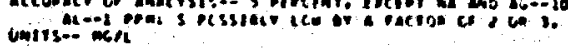

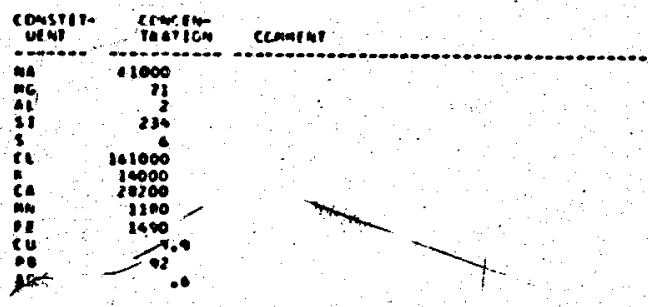

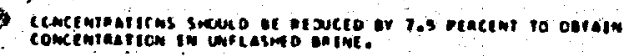

ereciocenomic bate

souncis-

mitman is

morement is

rectson"
Fingen:

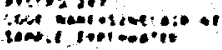

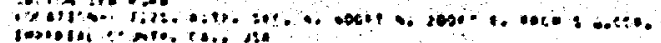

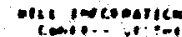

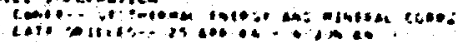

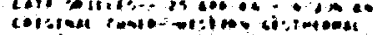

n!! ton?

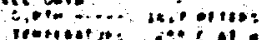

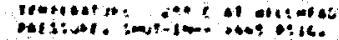

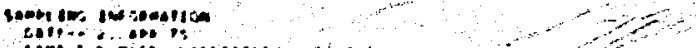

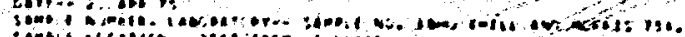

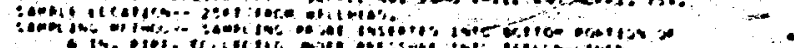

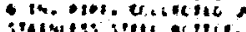

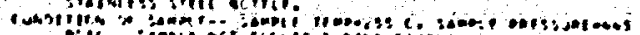

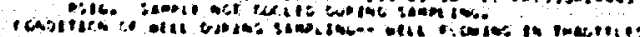
celoving

morsica gats

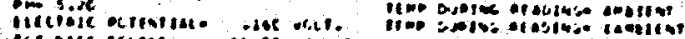

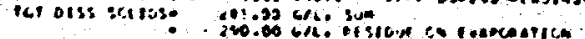

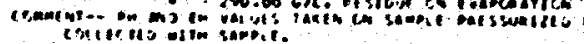

mine bate.

2

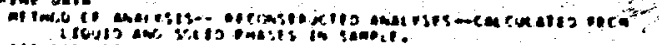

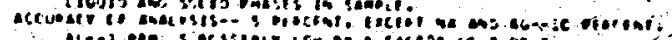

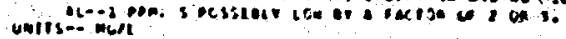

cansill- ciarions

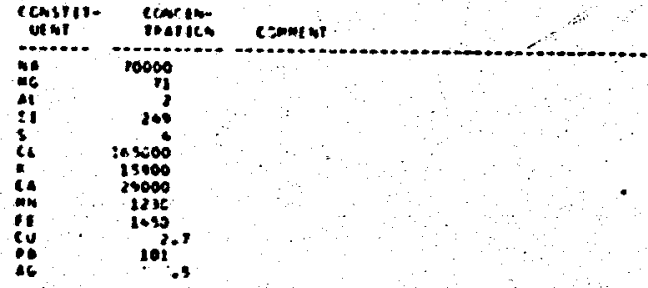

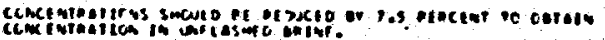

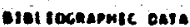

scuricts-s

cusing iso

mescests

nuthetien 


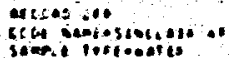

ats state.

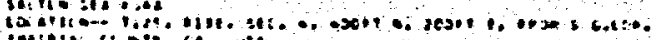
ofie tomatica

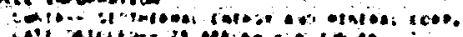

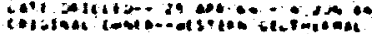

nil one

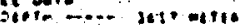

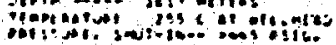

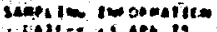

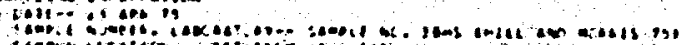

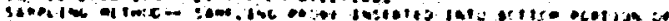

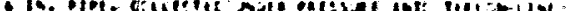

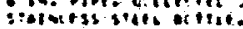

ace oure

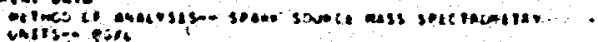

restil. concin

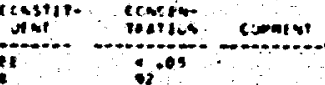

it

is

is

$\operatorname{lin}_{i=1}$

ii

si

i.

a

10

in e

if

is

î

8

su

if

In

${ }_{\mathrm{N}}$

M.

if

if

of

if so

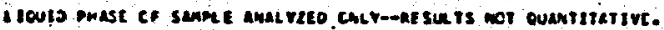

eleziochapric onta

Soukce s-

milt is

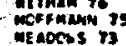

Metcescus

asco cansingent

mase constivent

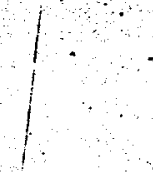

$-1$
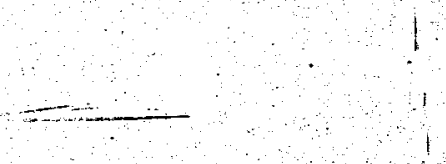$$
\text { (1) }
$$

69

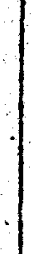

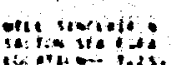

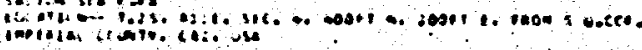

ofit irconition

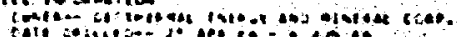

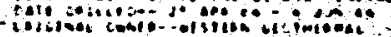

Wil oute

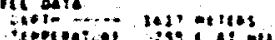

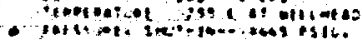

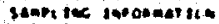

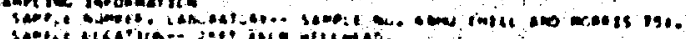

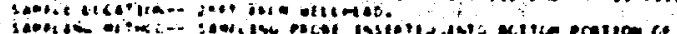

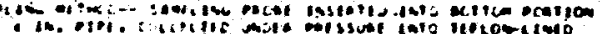

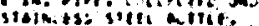

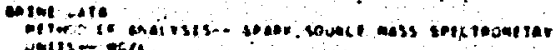

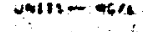

cinstite cincets

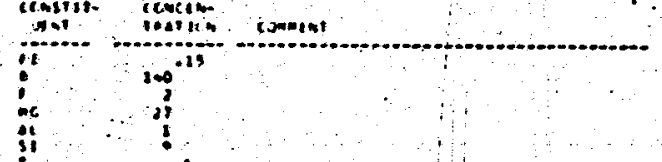

if $\cdot$

if $\quad 304$

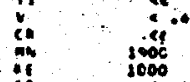

ii. $\quad .0900$

in

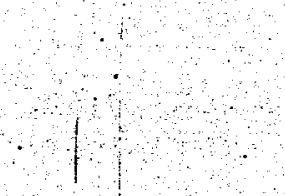

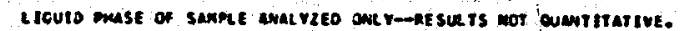

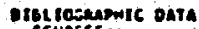

scuaces-

mitren 76

merpous is

MELCEsen 60 


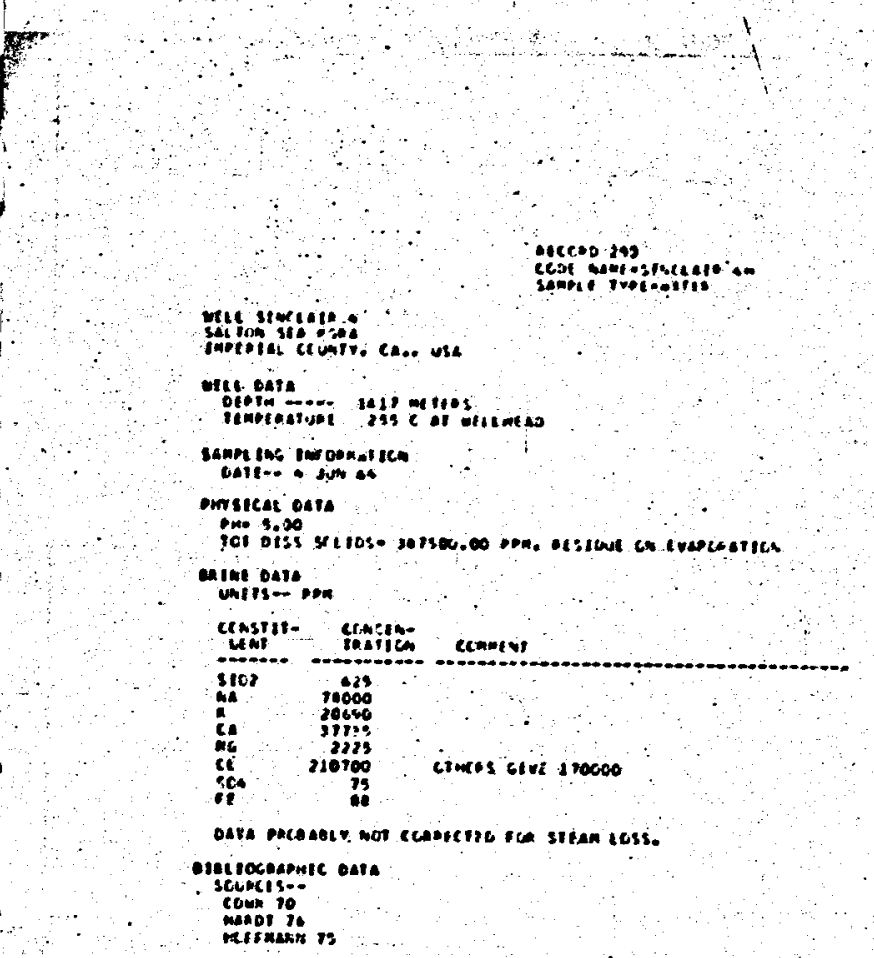


(c)ixtelt.

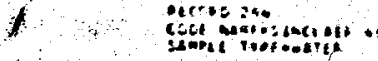

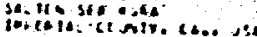

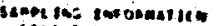

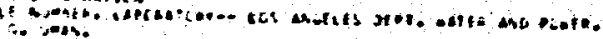

esiar gite

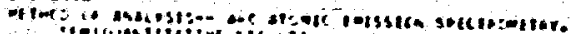

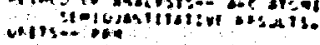

topste- crobio scoms

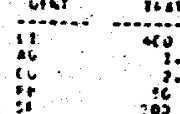

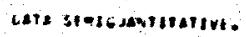

ithisckoamic oart

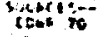

1
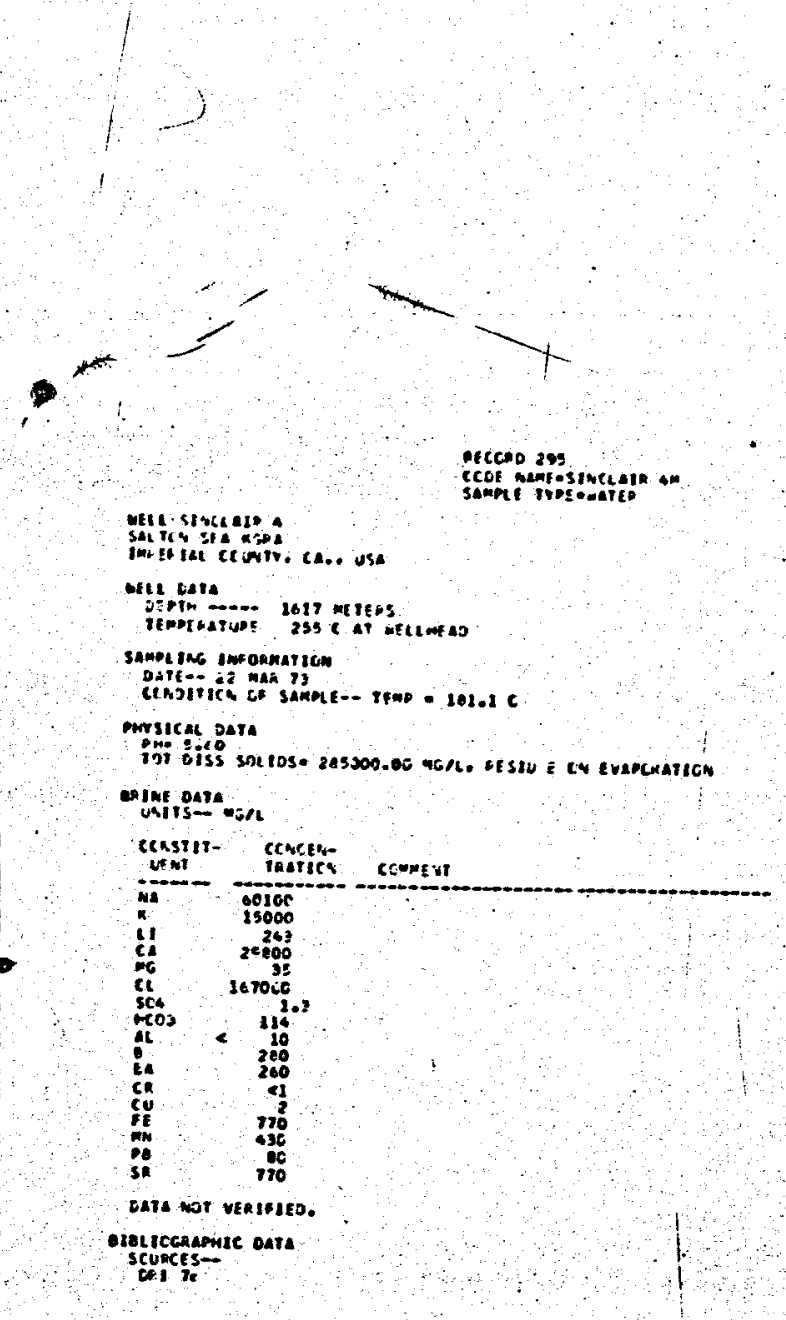

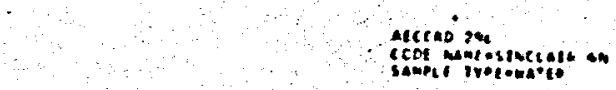

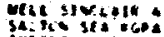

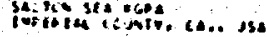

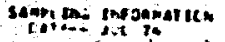

minivinge

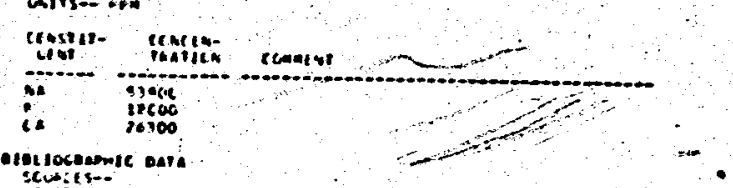

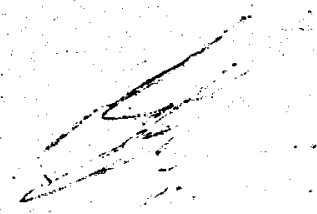

mete sixisen.

impenisc cowiviti. ca.. usa

sinnivic mecanalion

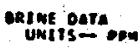

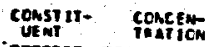

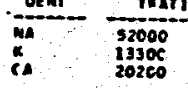

Hesurocenomic onsa

scuncers-7s 
semat irrionatis

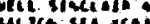

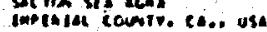

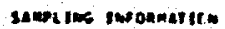

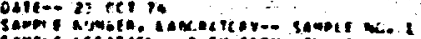

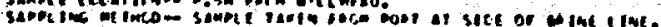

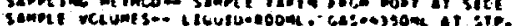

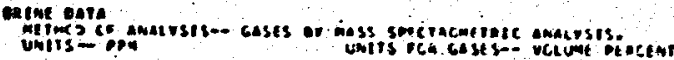

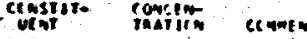

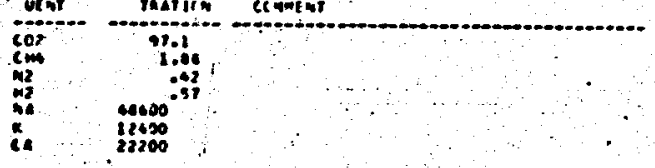

Gas andirsis oces not incluof olsscivio casts.

erenicciamic bata scuacts-;

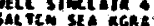

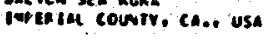

\section{$\operatorname{cotcoso} 200$}

ccot maxtes inceeln of

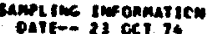

Patr- 23 aci 13

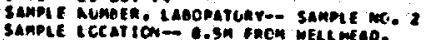

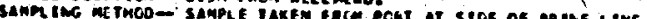

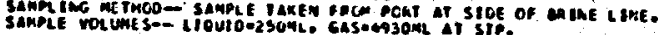

\section{eximgibars.}

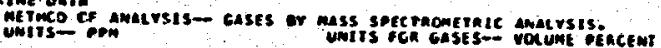

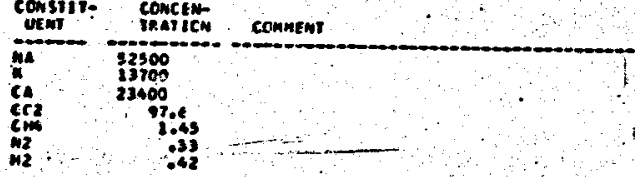

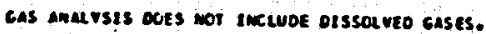

elezgcienomic Data souncessis is 


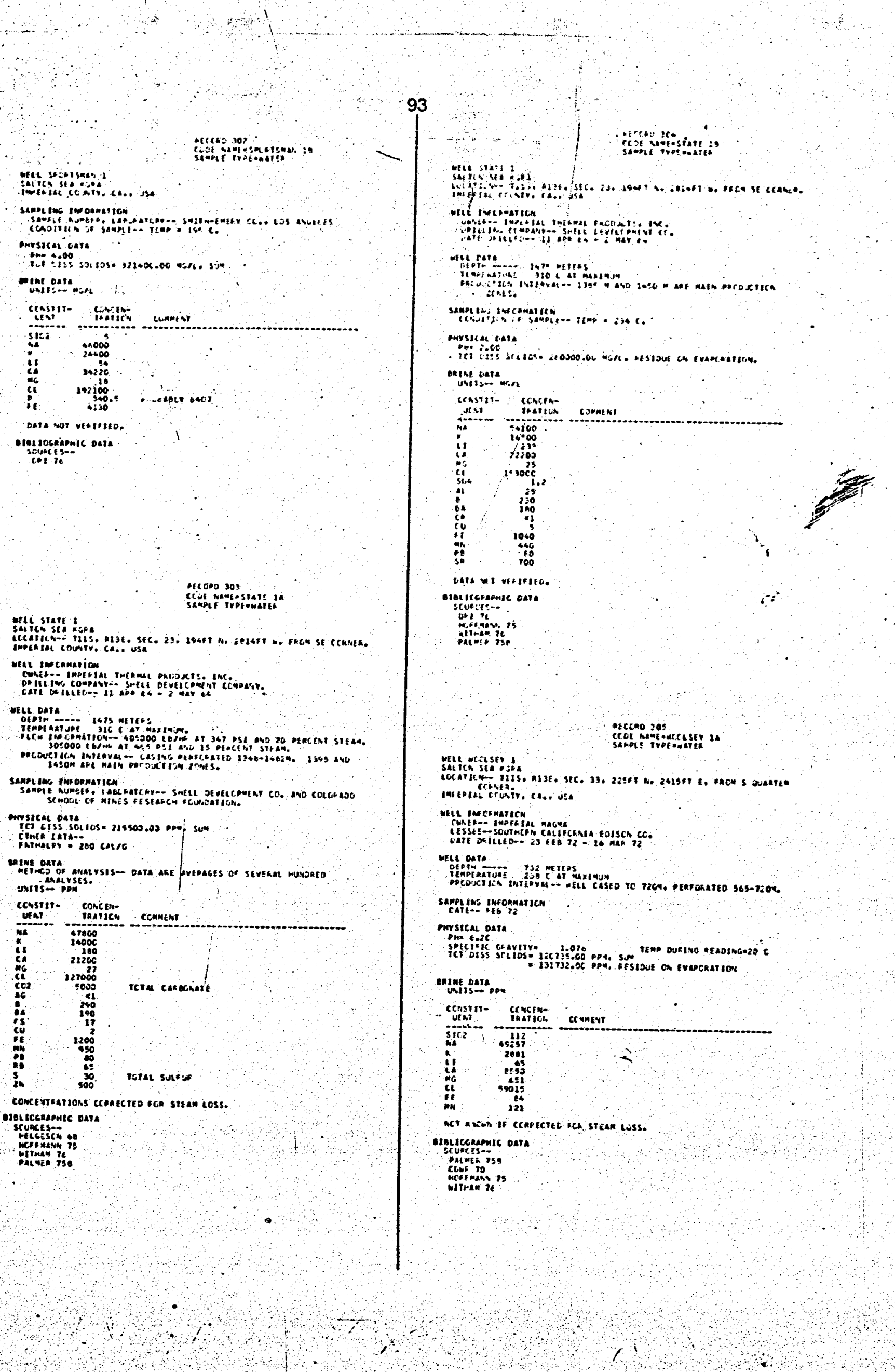




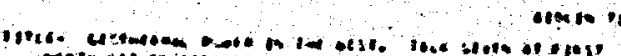

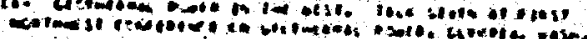

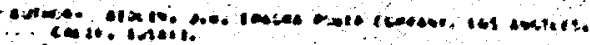

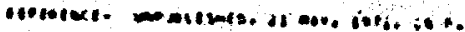

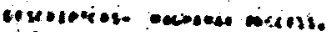

arention is

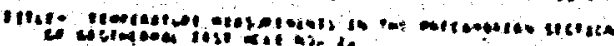

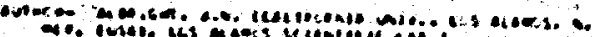

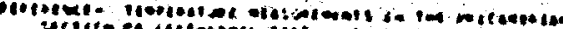

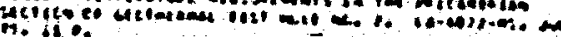

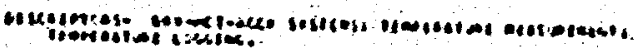

aivin

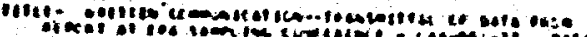

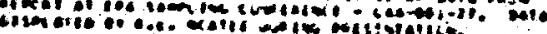

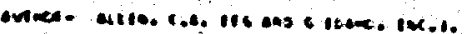

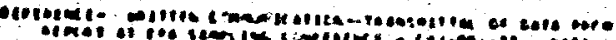

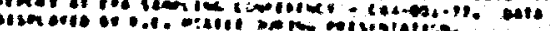

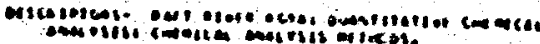

enisa $n$

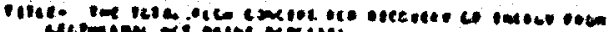

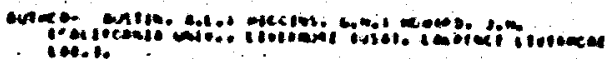

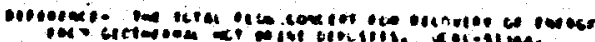

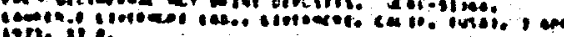

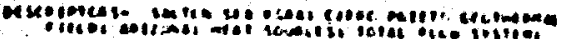

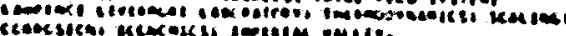

ine ife is

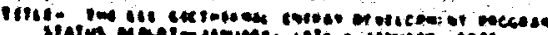

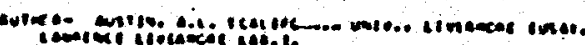

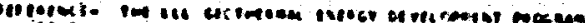

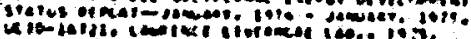

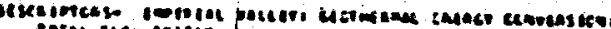
cata nce sosite

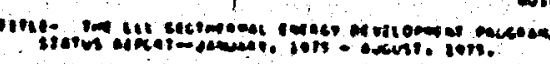
wition ne

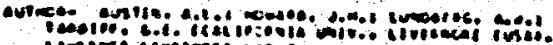

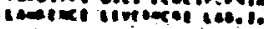

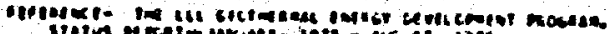

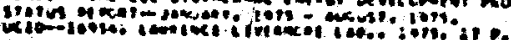

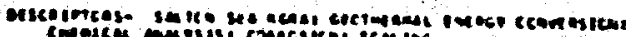

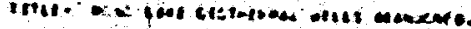

antal

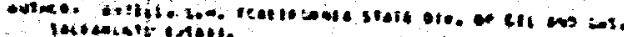

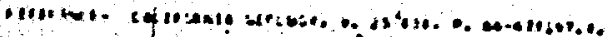

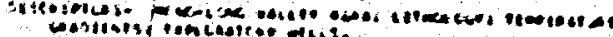

O coment n

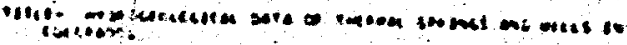

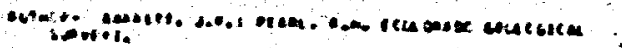

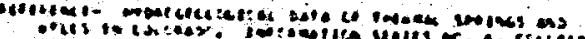

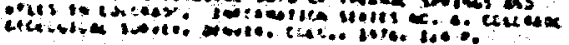

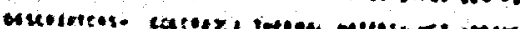

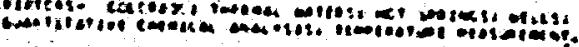

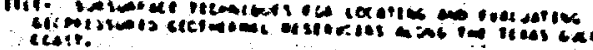

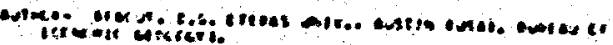

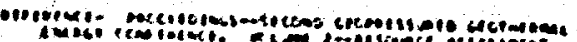

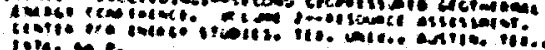

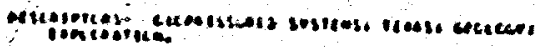

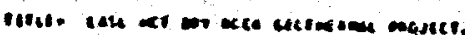

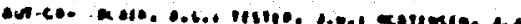
icavienie

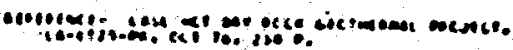

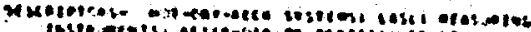

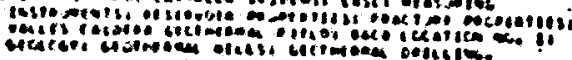

nest is

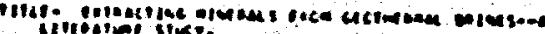

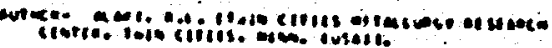

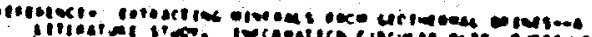

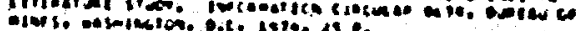

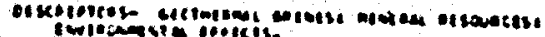

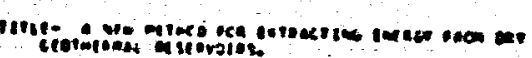
encos is

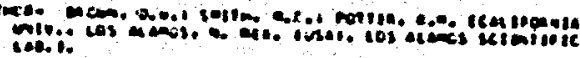

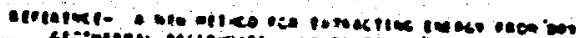

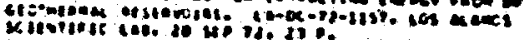

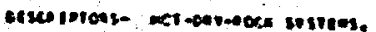




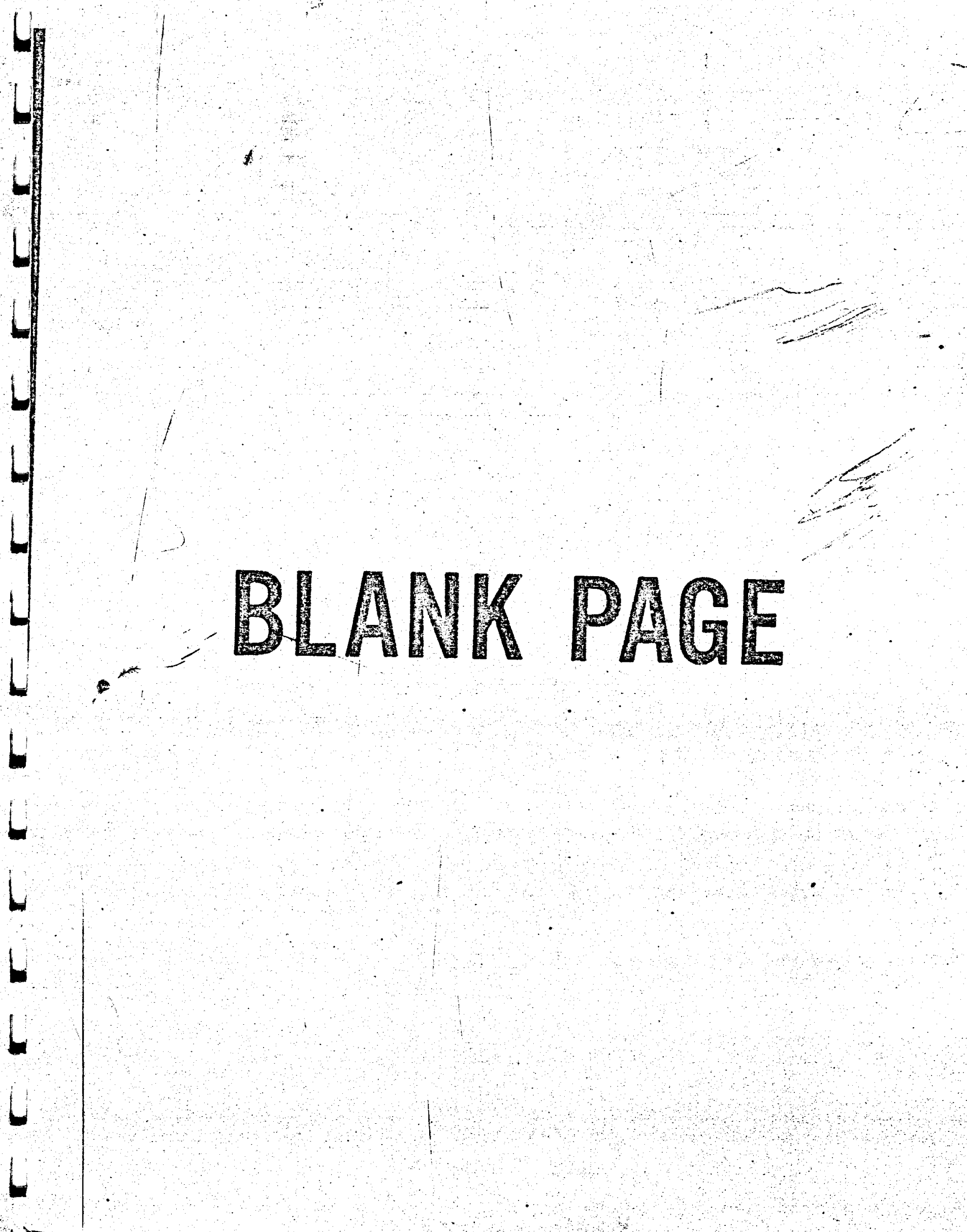

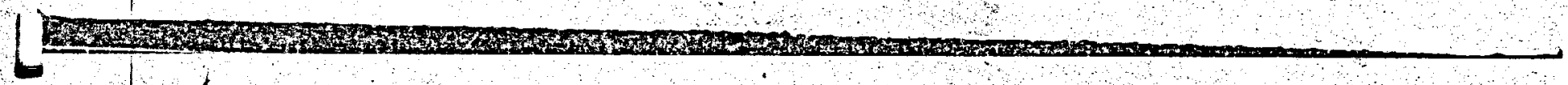
) 


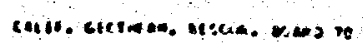

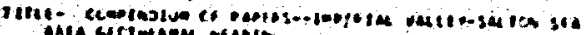

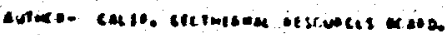

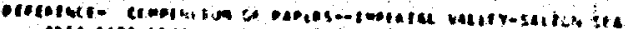

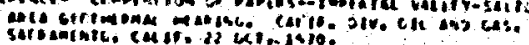

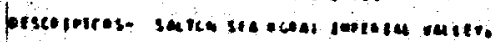

1

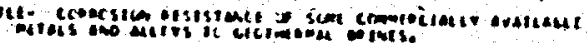

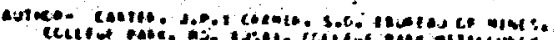

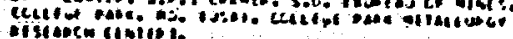

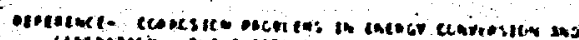

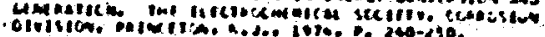

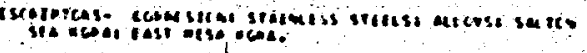

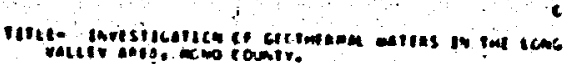
cointior

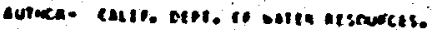

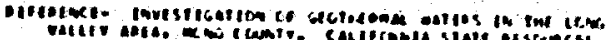

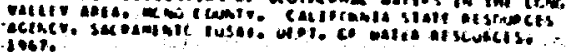

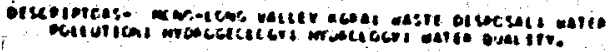

cone is

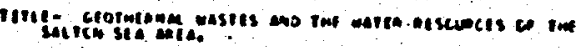

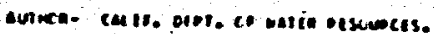

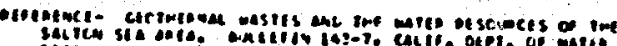

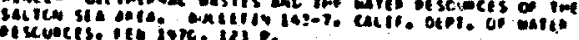

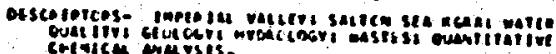

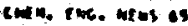

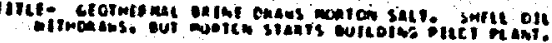

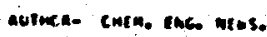

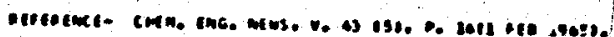
orscojotcas- saren ste agas:

cotemin :

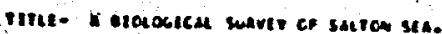

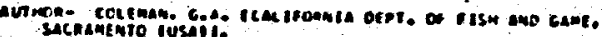

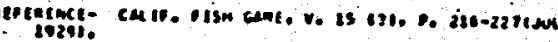

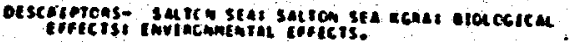

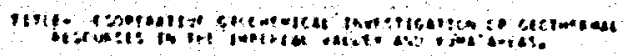

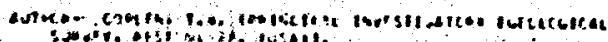

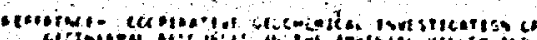

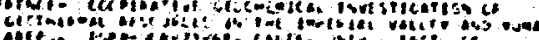

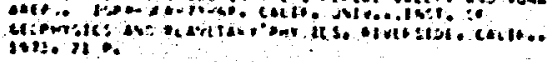

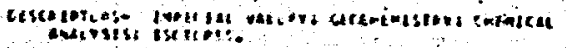

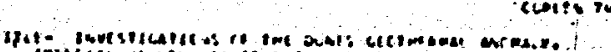

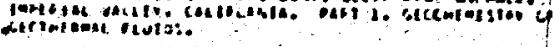

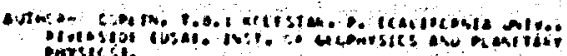

$$
\text { onvilest. }
$$

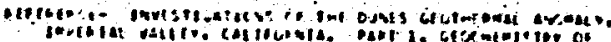

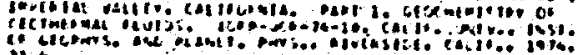

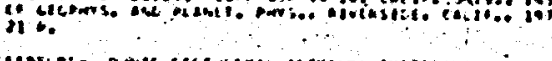

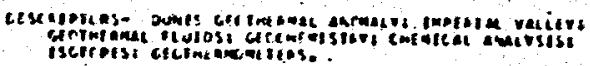

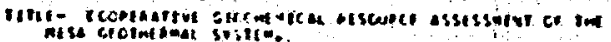

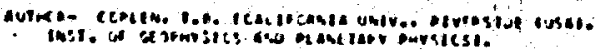

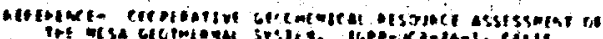

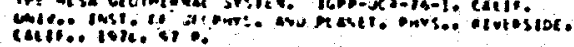

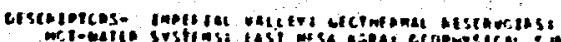

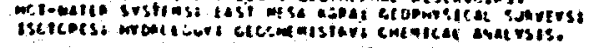

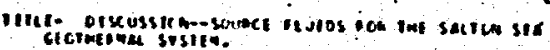
Costc so

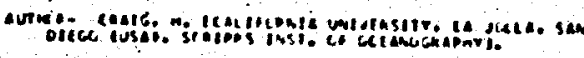

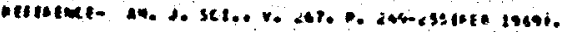

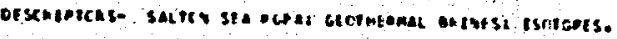

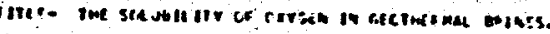

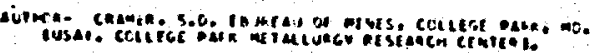

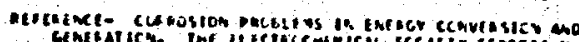

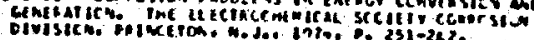

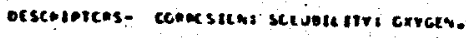

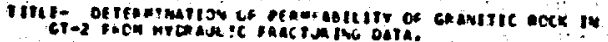

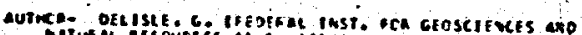

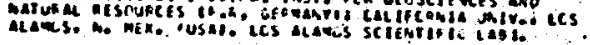

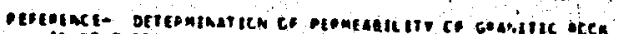

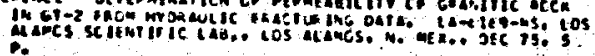

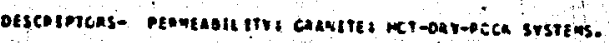




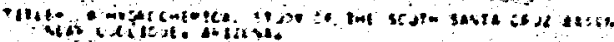

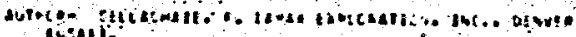

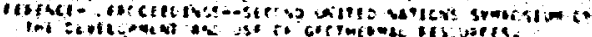

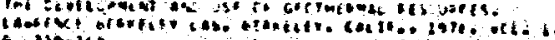

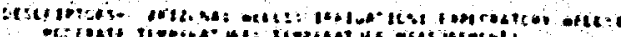

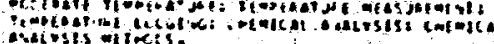
its micess.

tives to

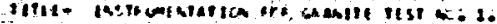

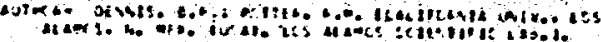

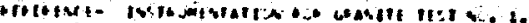

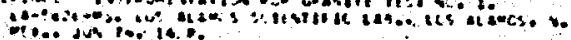

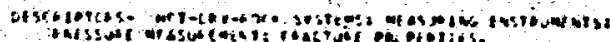

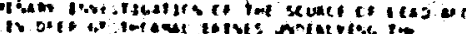

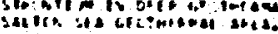

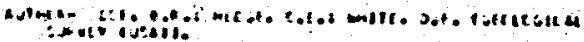

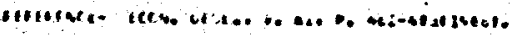

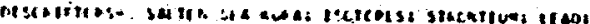

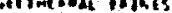

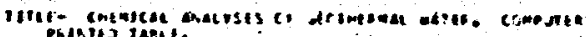
endanis ineat.

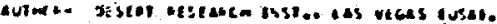

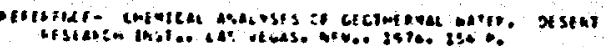

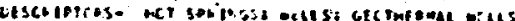

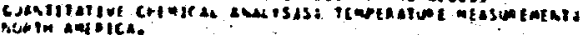

$\therefore$ vitenga 12

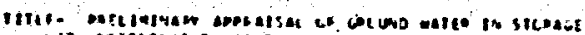

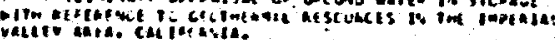

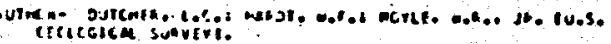

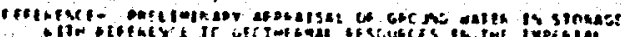

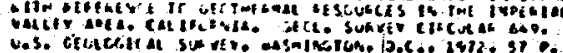

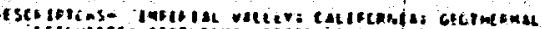

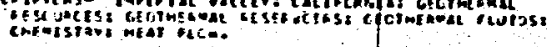

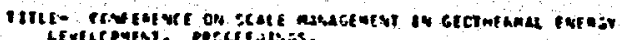

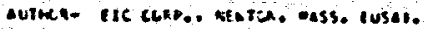

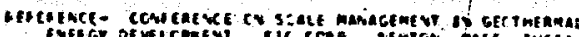

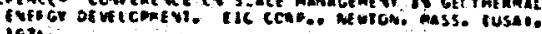

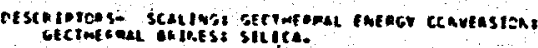

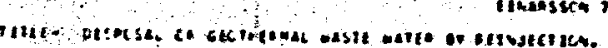

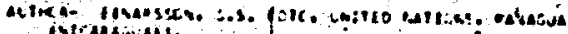

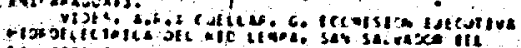
saliatel

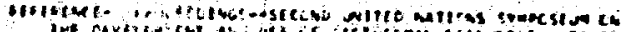

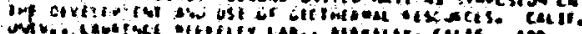

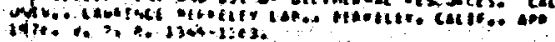

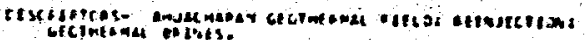

licis of

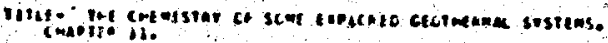

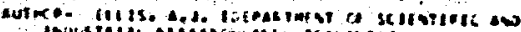

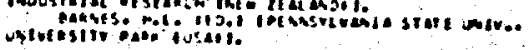

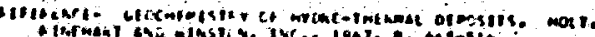

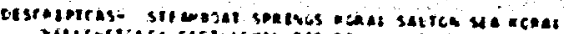

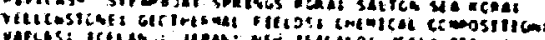

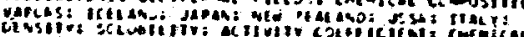
cullicalivin.

Eac. alk, Jo es

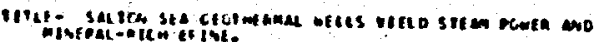

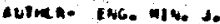

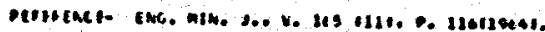

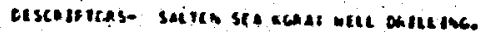

\section{ton to}

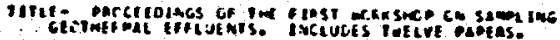

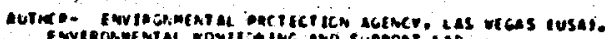

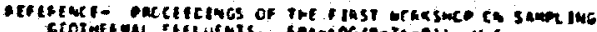

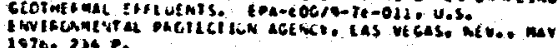

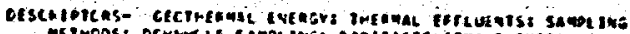

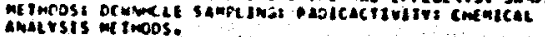

torel zea

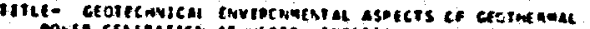

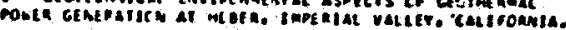

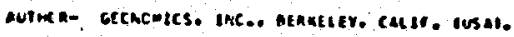

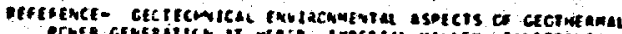

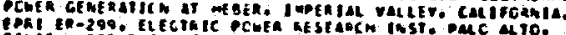

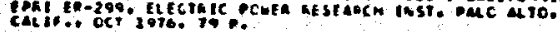

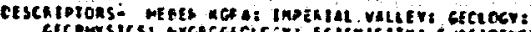

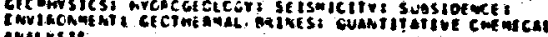
avitisis.

EoAl 700.

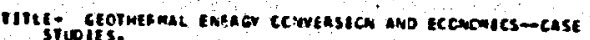

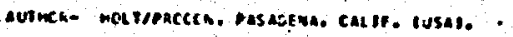

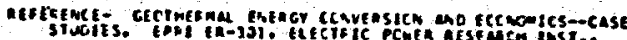

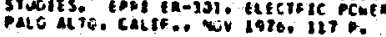

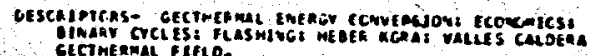




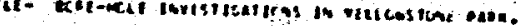

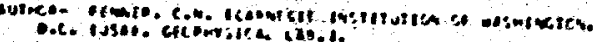

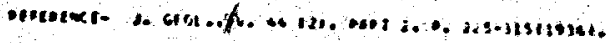

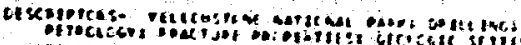

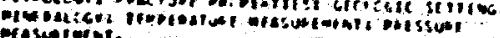

ecuanter es

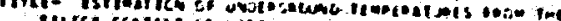

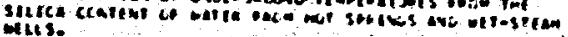

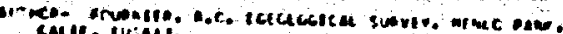

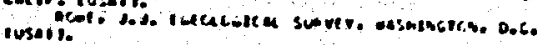

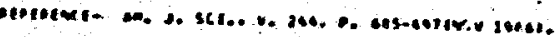

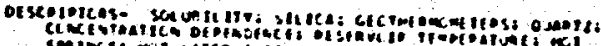

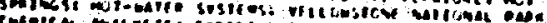

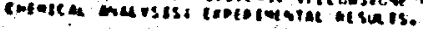

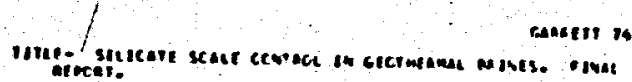

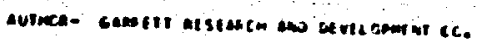

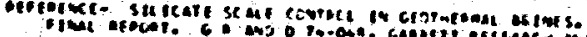

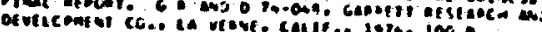

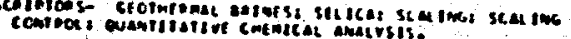

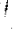

Gans 1007

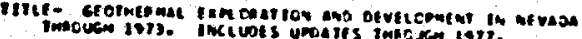

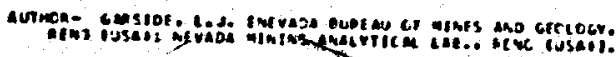

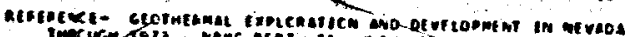

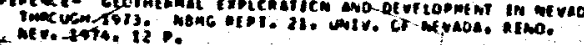

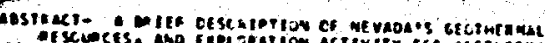

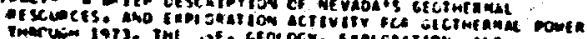

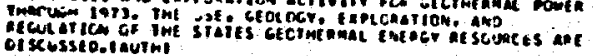

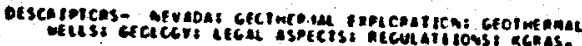

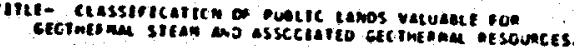

coosing it

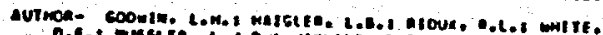

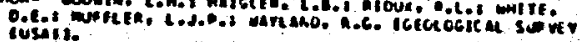

ateceence

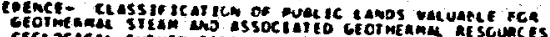

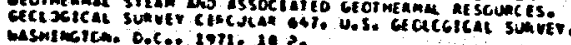

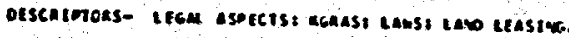

cotositin it

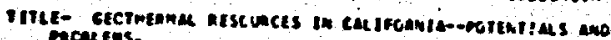

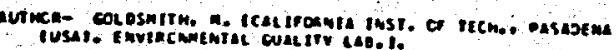

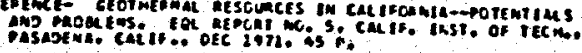

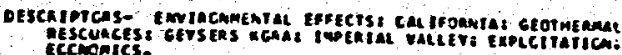

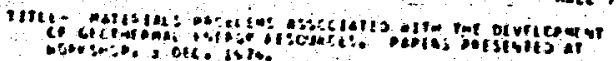

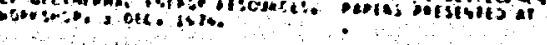

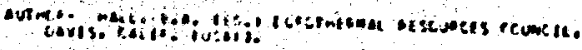

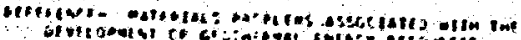

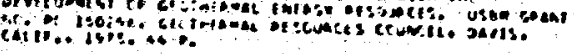

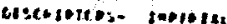

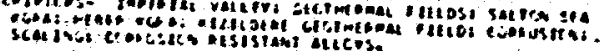

and 10

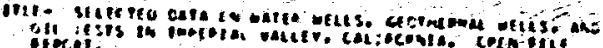

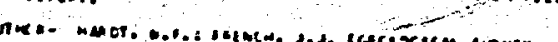

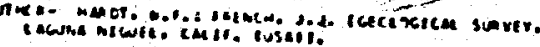

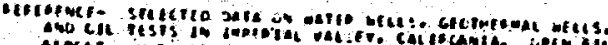

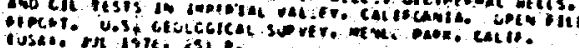

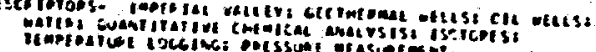

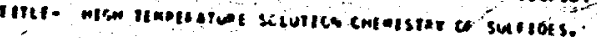
auinca- melceseno moc. . . . .

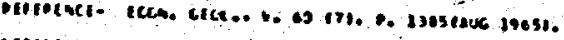

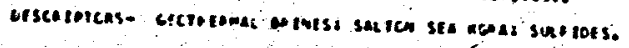

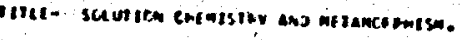

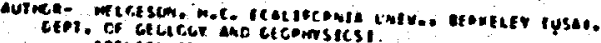

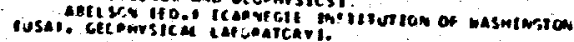

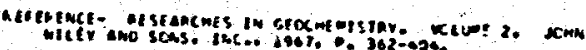

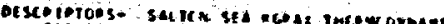

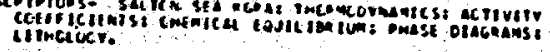

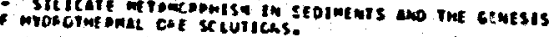

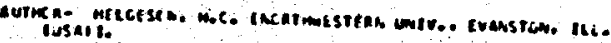
eirun. sos, deto.t

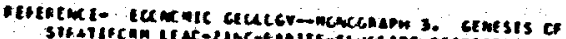

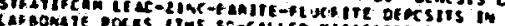

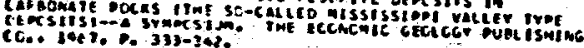

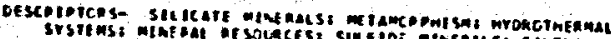

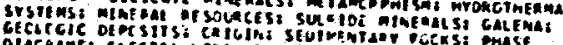

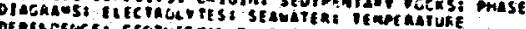

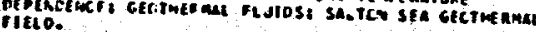

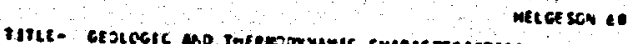

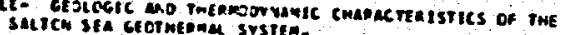

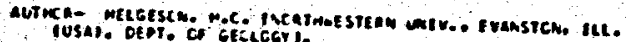

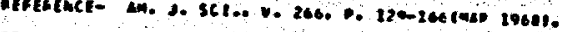

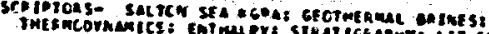




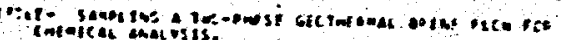

isi. is

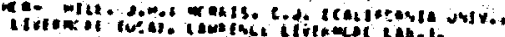

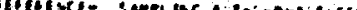

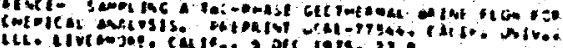

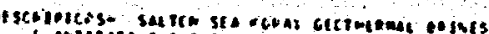

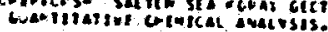

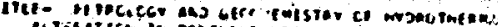

mricesso to

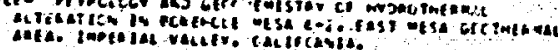

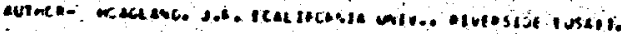

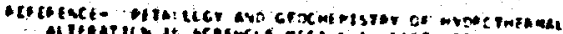

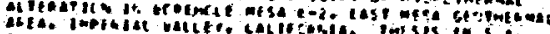

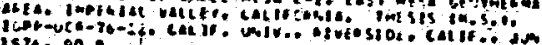

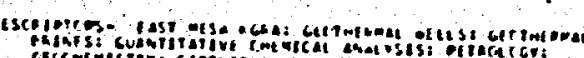

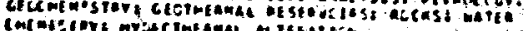

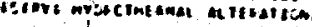

Mablayo vel

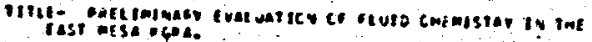

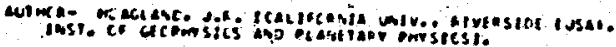

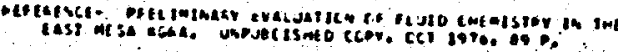

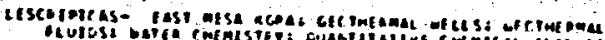

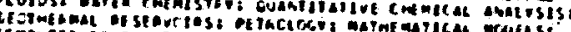
cconite cackaricas.

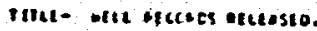

$\operatorname{mogsen} 14$

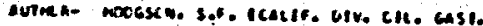

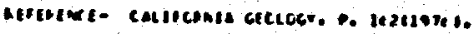

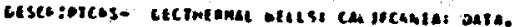

mefoinater is

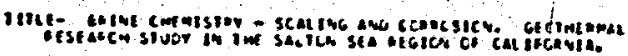

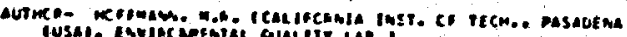

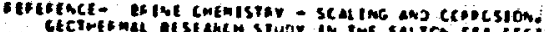

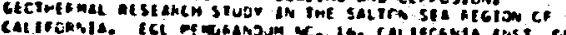

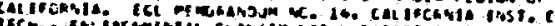

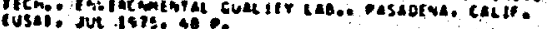

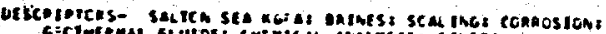

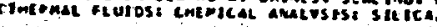

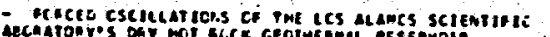

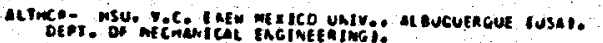

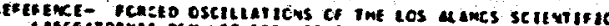

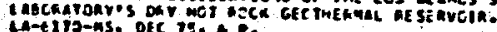

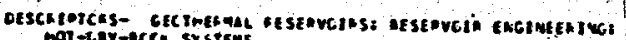
mot - Lar-ecch, srstems. 


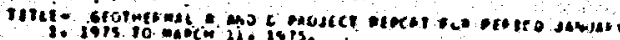

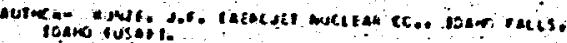

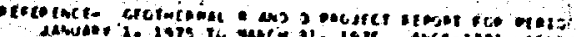

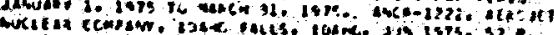

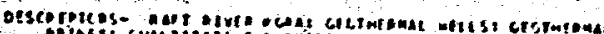

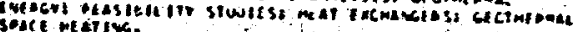

aviet to

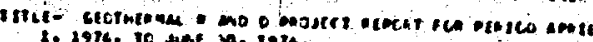
1. 1976. Th that 30. 1975.

autuce- Mimite det. Ho.t

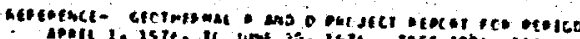

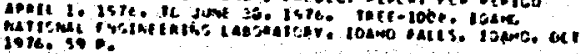

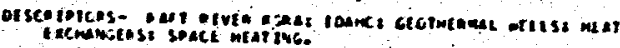

Ant: 10

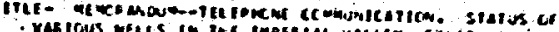

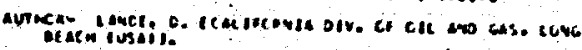

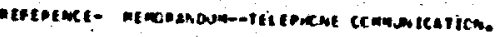

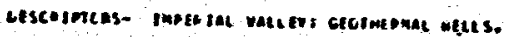

cater it

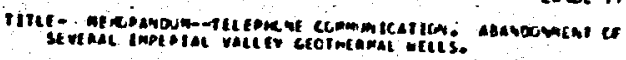

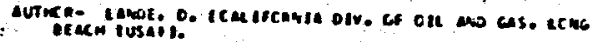

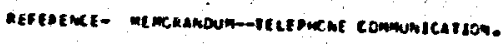

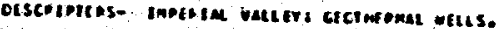

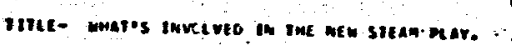
cawatuce

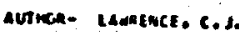

Ref ERf uce- OIl cas do. P. e0-t 3creb 10, 19641.

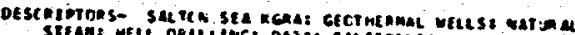

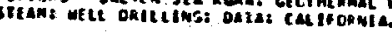

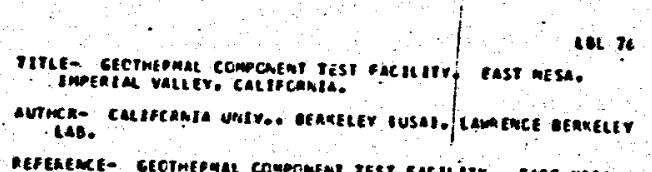

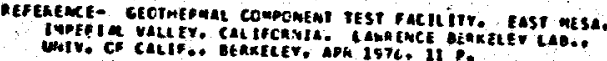

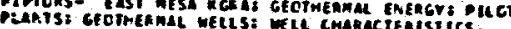

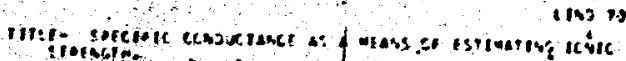

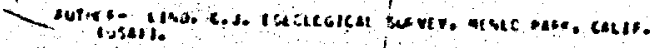

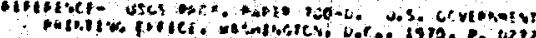
$062+2-6243$.

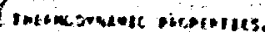

cesantes es

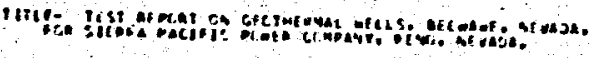

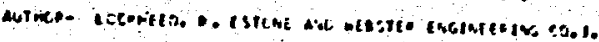

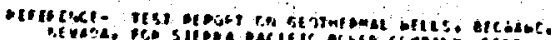

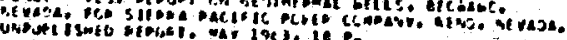

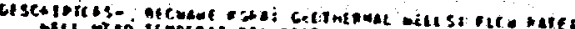

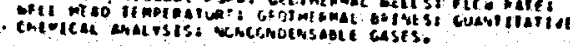

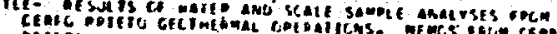

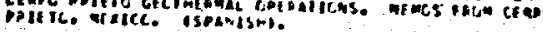

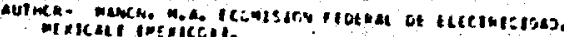

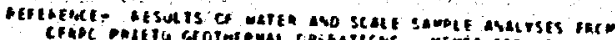

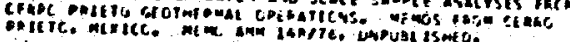

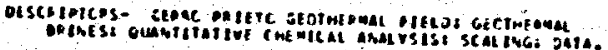

maturias $x$

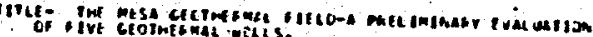

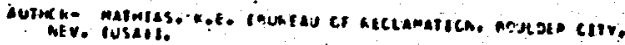

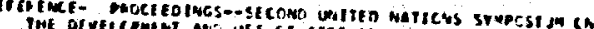

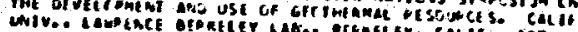

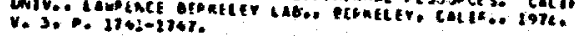

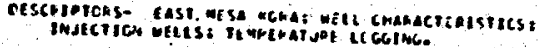

mating as nea

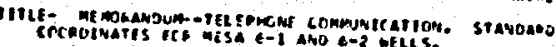

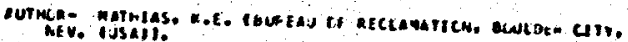

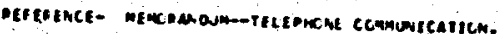

Descaintons- casi wesa remi.

arise 7

Ditlf- cclefsticin ce oata sampl ing smetis, Reft ange

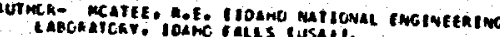

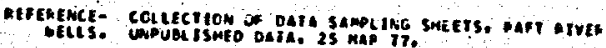

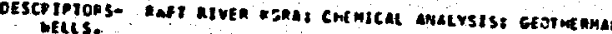
wetis. 
mucta is

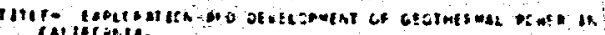

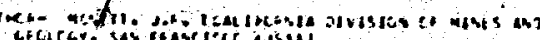

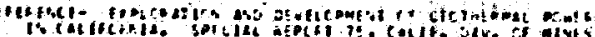

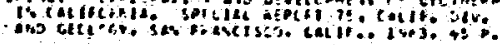

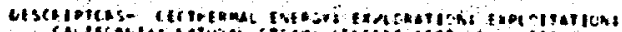

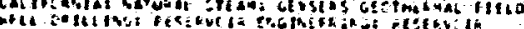

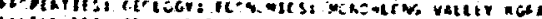

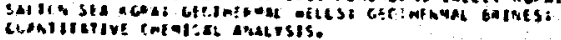

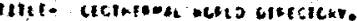

waces is

A.7mese meaves, 0.1. 180.0

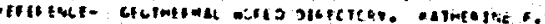

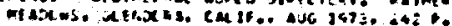

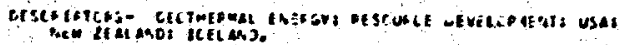

$j^{2}$

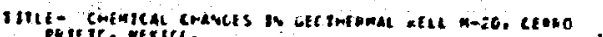

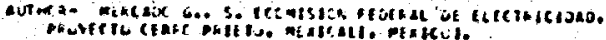

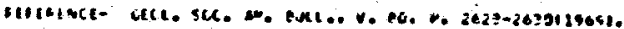

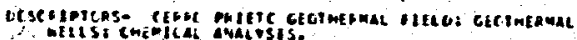

NE HeCACE is

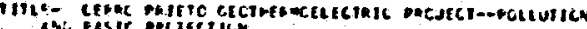

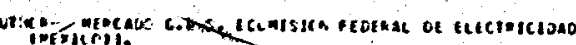

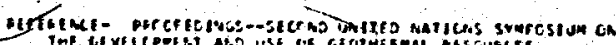

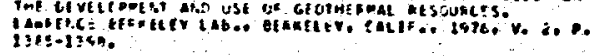

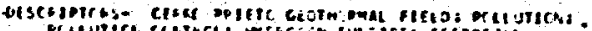

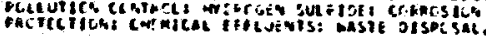

ag acADS is

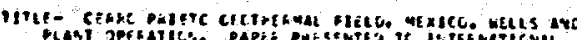

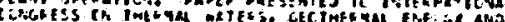
wilcoils if to

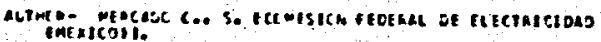

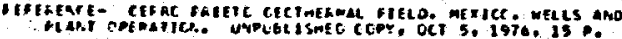

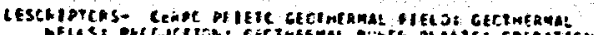

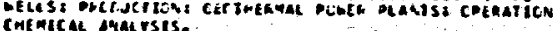

Nigoreten al

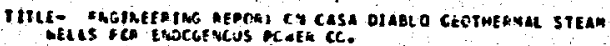

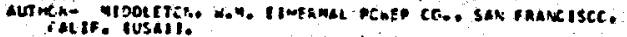

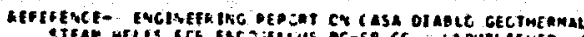

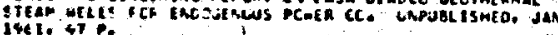

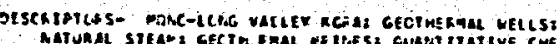

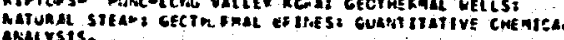

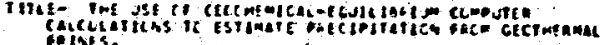

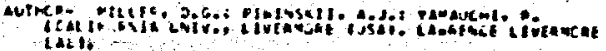

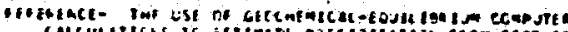

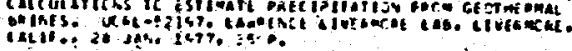

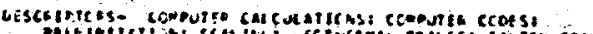

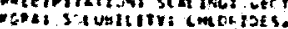

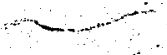

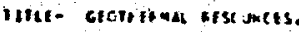

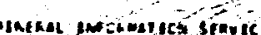

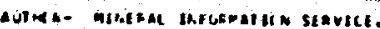

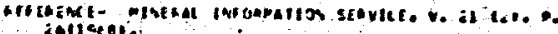
inlicento

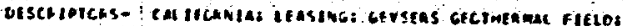

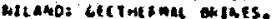

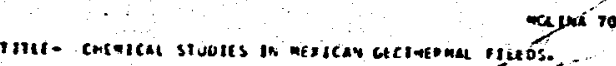

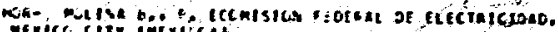

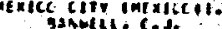

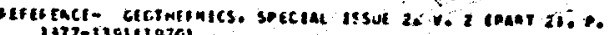
II1-1391119701.

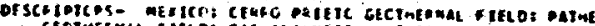

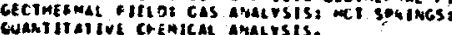

methen b9

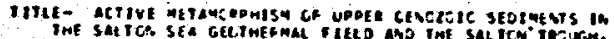

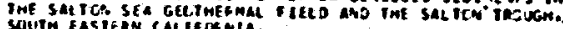

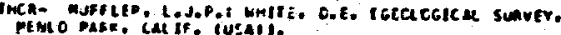

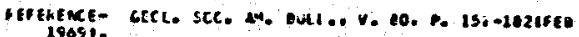

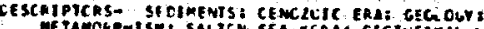

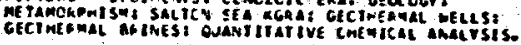

cuen to

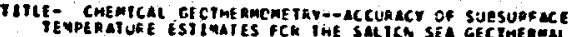
mato.

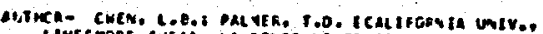

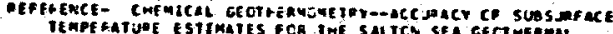
THPF FAT J E ESTIMATES FCR THE SALTCA SEA GECTHERMAL

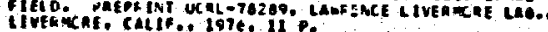

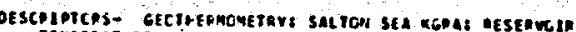

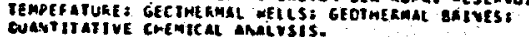

Datmen isa

TITIE- CECIHETMAL CEVECCOMEMI CF IME SALICA TRCUCM.

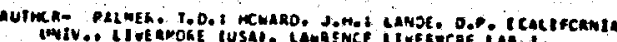

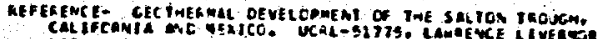

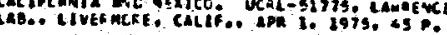

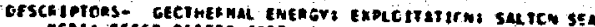

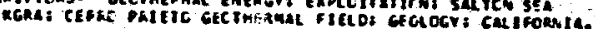




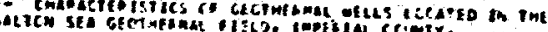

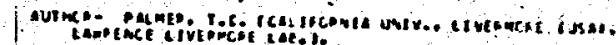

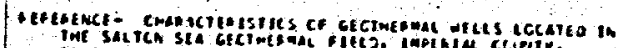

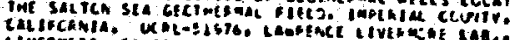

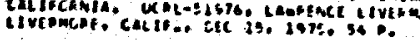

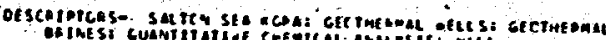

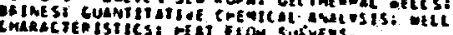

bazara nec

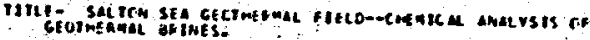

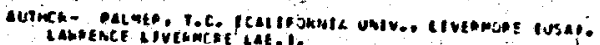

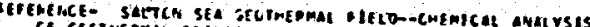

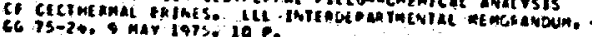

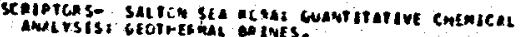

iinic marazan vol

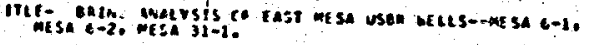

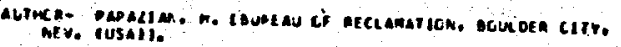

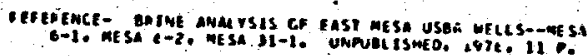

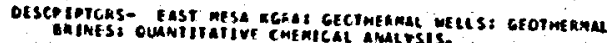

caprazian vos

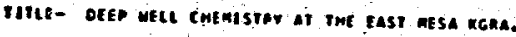

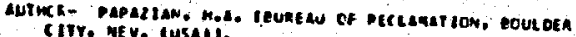

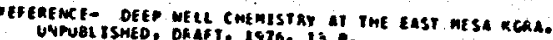

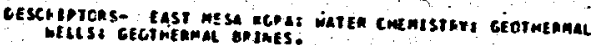

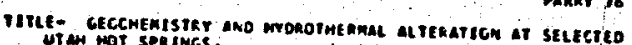

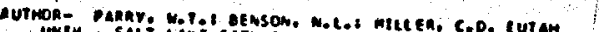

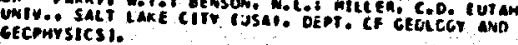

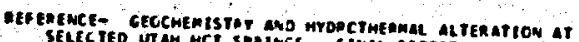

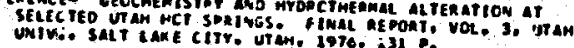

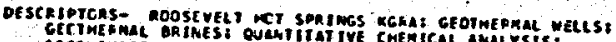

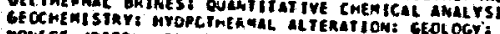

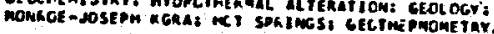
manar rea

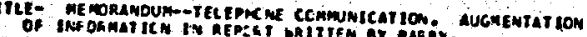

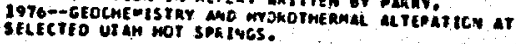

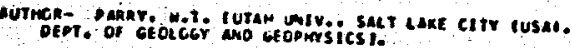

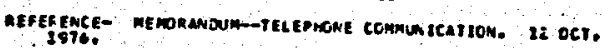
3sto.

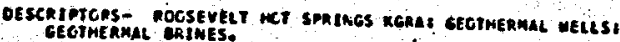

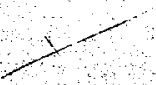

Demill 78 omim 2

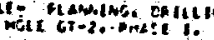

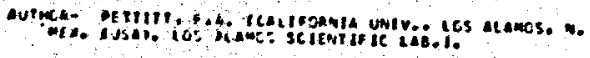

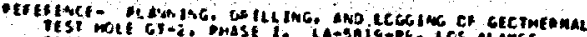

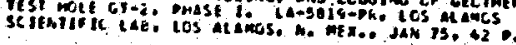

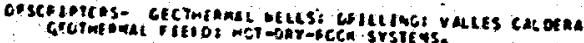

ottitif 75

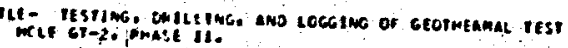

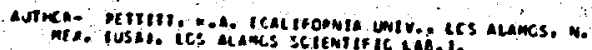

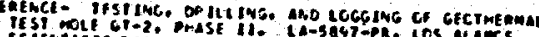

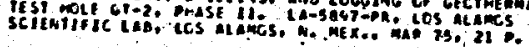

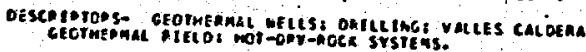

mittios 10

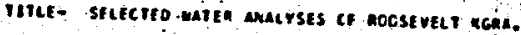

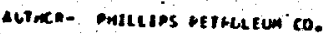

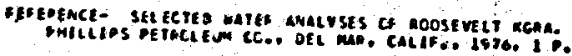

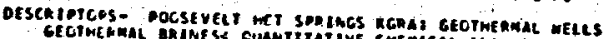

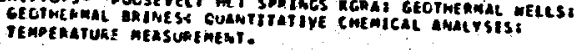

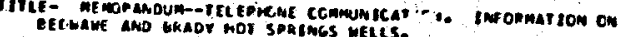

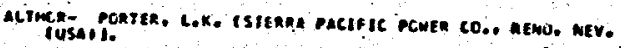

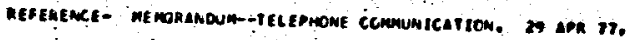

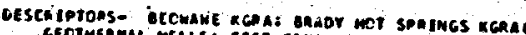
GEOTMEOMAC WELSi GeCTHERMAL ORINES.

Dusrrmun 74

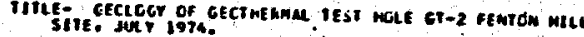

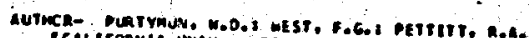

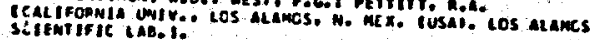

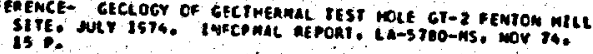

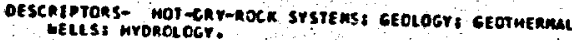

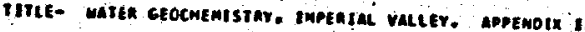
AUTHOR- REEO, Mod.

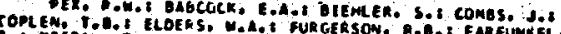

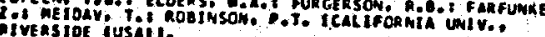

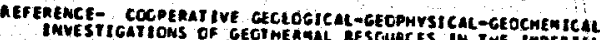

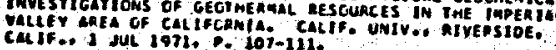

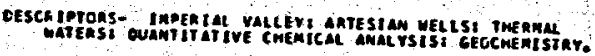




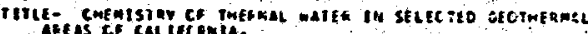

sets $\mathrm{re}$

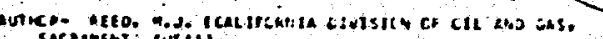
azmenti cusali.

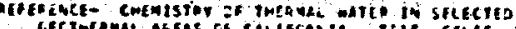

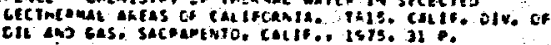

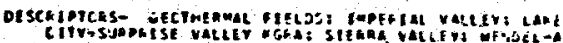

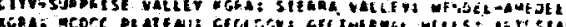

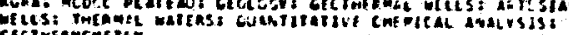

ta:0 re

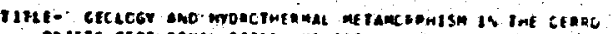
on ie ic ce ctmesmai dietro, we $x$ ice.

alraca- Aeto. mode trablicaris wive alveaside cusas:

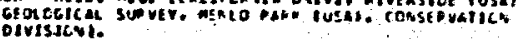

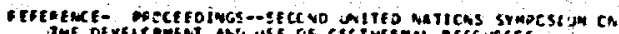

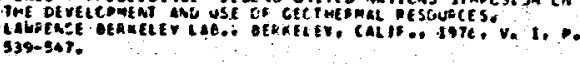

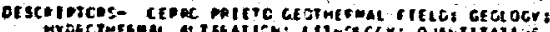

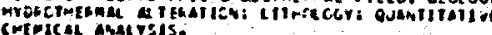

ą $\times 7$

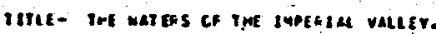

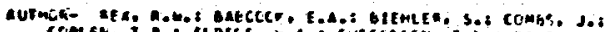

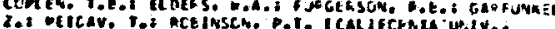
Rivisiog iusaid.

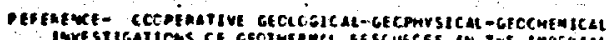

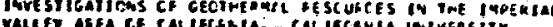

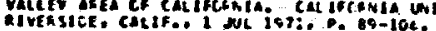

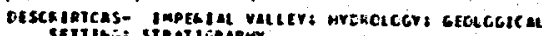

SAMOERS 71

TIILE- MINEPA CCATENT OH SELEC TO GECTMERMAL wATERS.

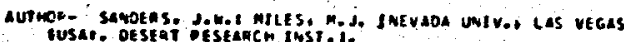

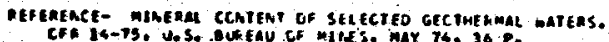

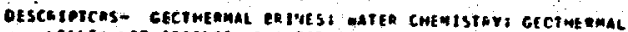

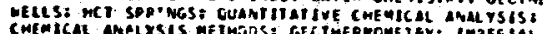

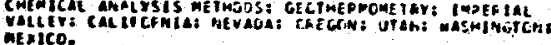

seimirt it

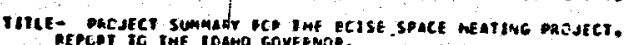

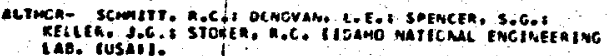

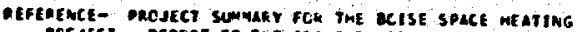

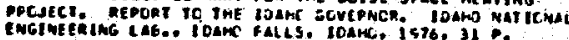

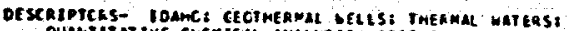

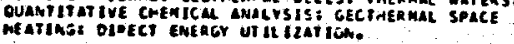

SAUNE 76

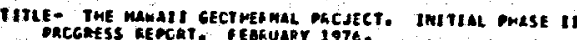

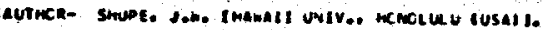

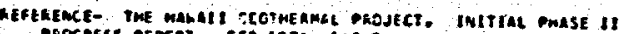

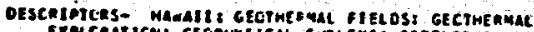

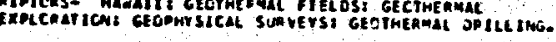

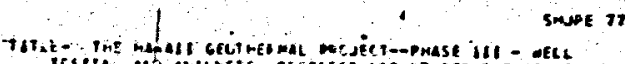

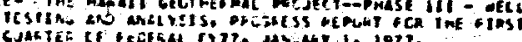

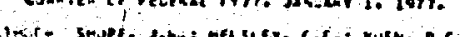

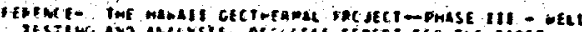

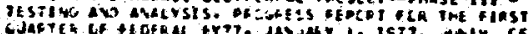

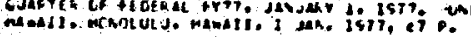

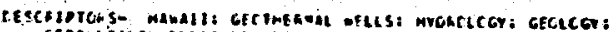

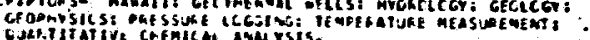

Sanvere es

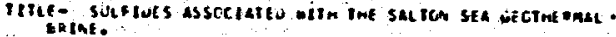

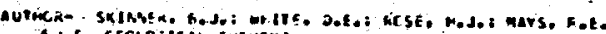
1 J.S. CECLOZICAL SUEVETI.

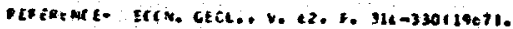

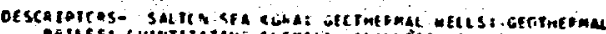

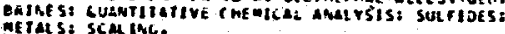
i

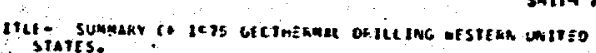

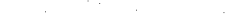

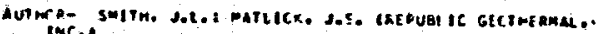

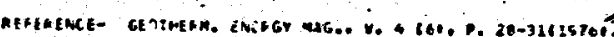

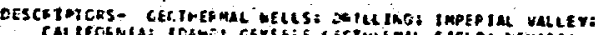

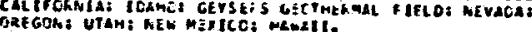

Sniten 7

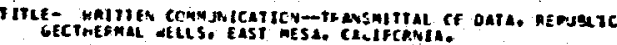

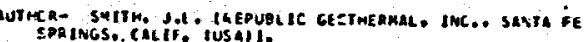
ECIREACE- DPITIEN CCAYUNICATIEA-TRAR.SMITTAL CF CATS.

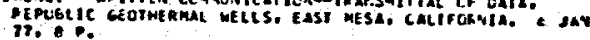

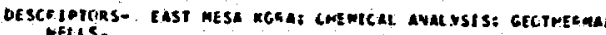

Soencek 7

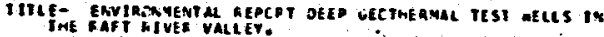

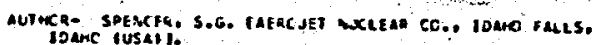

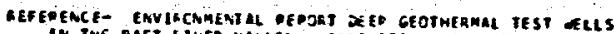

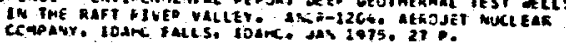

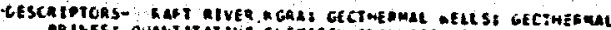

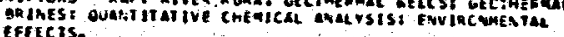

sivineas is

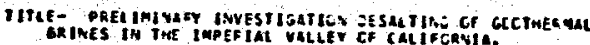

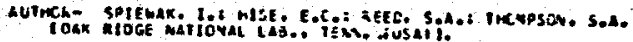
AEFEREM E- - PELLIMIMAEY IAVESTIGATIEM OFSALTING CE

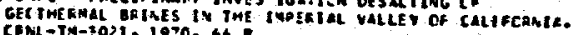

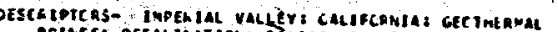

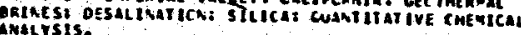




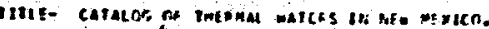

actira- suruestit bot.

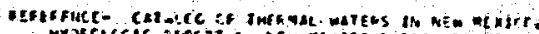

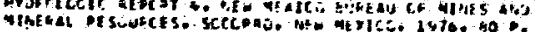

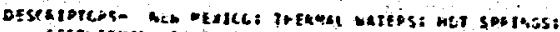

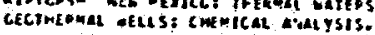

Sanstait ta

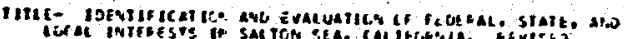
toriby.

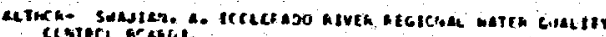

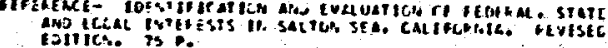

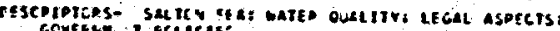
st receries.

1

vitr is

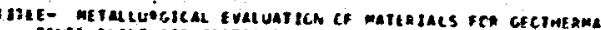
DCMTK PLANB AREL ReTJUAS.

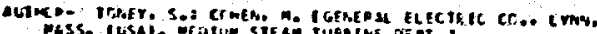

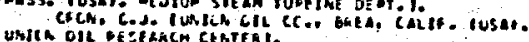

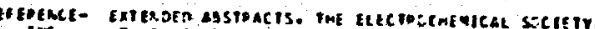

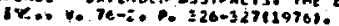

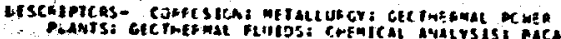

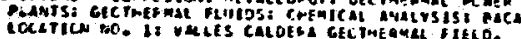

ICoet ris

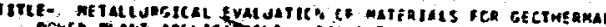

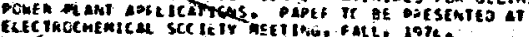

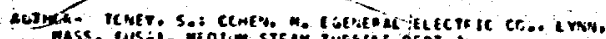

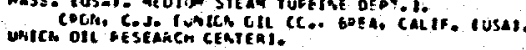

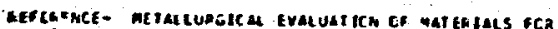

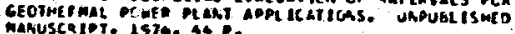

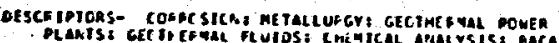

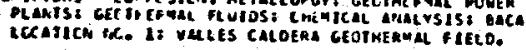

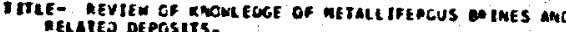

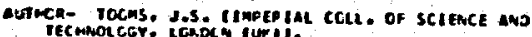

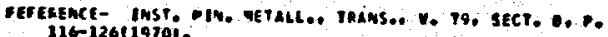
116-126119701.

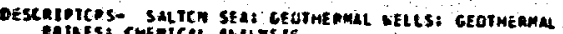

Thus sotil 74

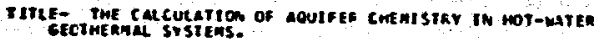

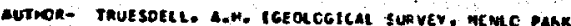

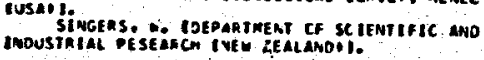

EEFEEACE - A. RES. U.S. CEOL S SUFV., V. 2 131, D. 72-27e 11974t.

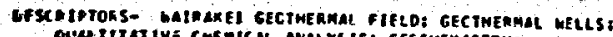

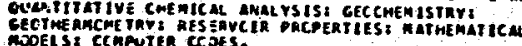

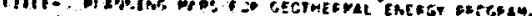

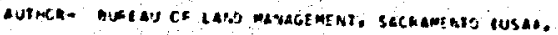

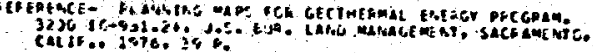

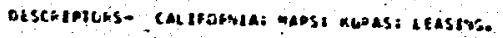

17ic- secono usan

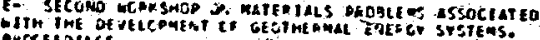
Puceedolacs.

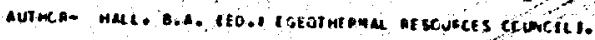

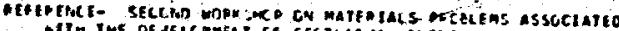

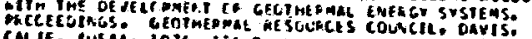

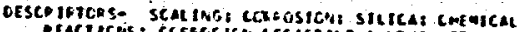

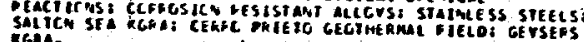
uses -16

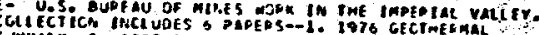

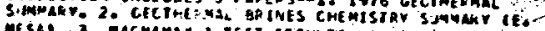

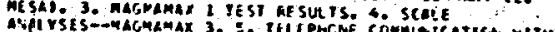

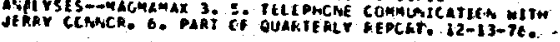

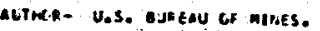

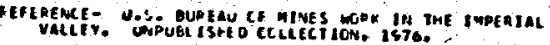

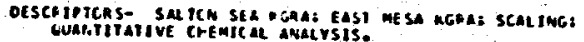

usen $n$

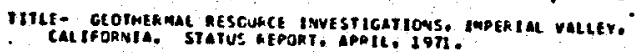

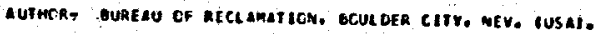

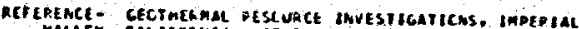

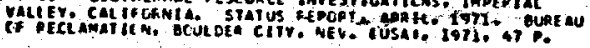

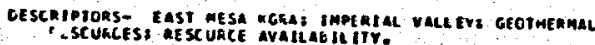

usap ne

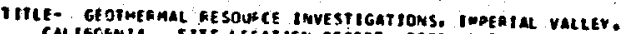
CALIFONMJA. SIIE LCCATICN AEPCRT. OEEP CECTMEAMAL IEST

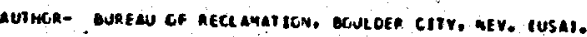

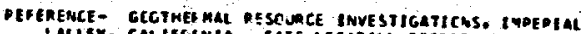

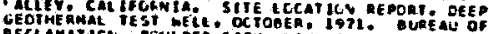

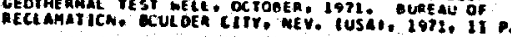

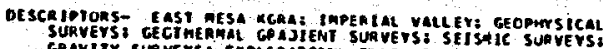

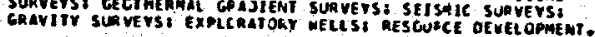

USBe 72

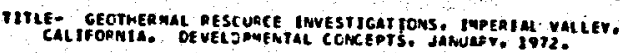

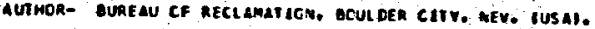
REREDENCE- CECTMERMAi AESTURCE INVESTIGATICNS. IMPERIAL VALLEY CaL IFOFAIA. CEEVEOPMENTAL CGNEPIS. JANUARY,

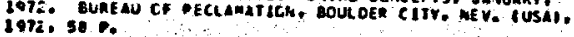

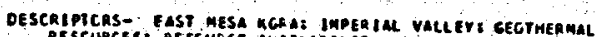

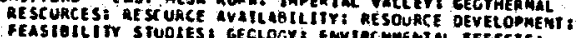
aESERVES. 


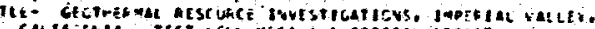

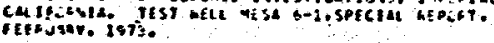

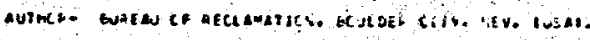

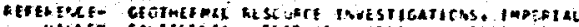

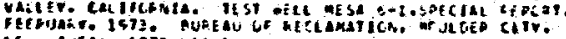

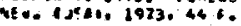

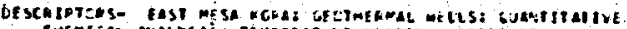

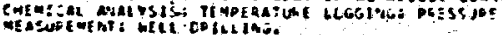

user 76

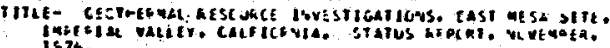

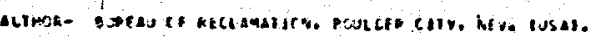

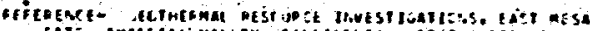

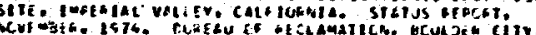

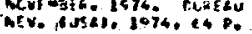

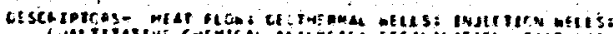

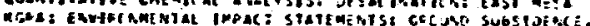

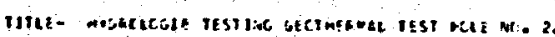
usst 7*a

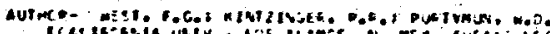

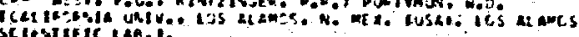

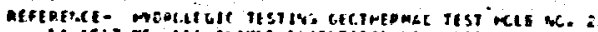

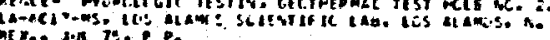

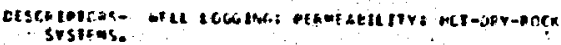

w5 58

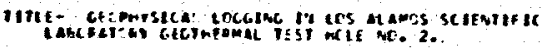

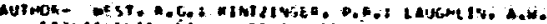

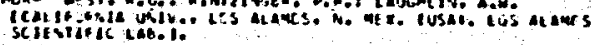

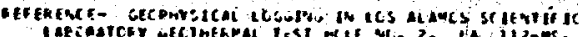

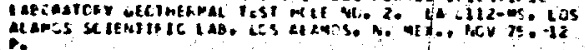

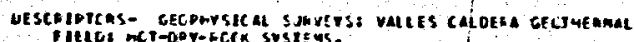

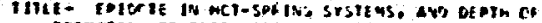

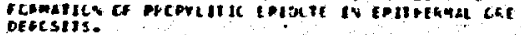

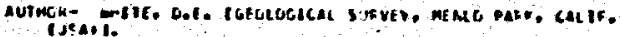

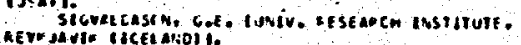

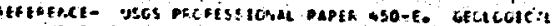

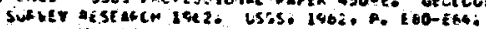

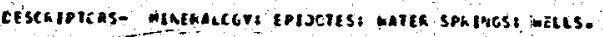

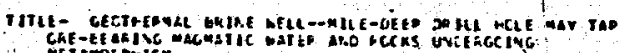

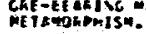

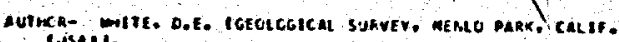

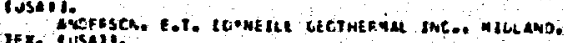

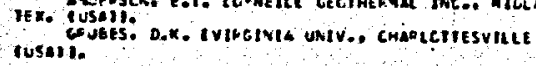

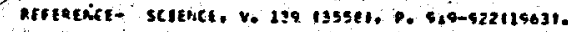

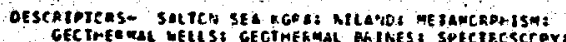

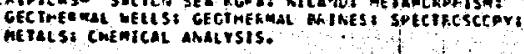




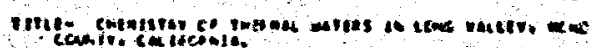

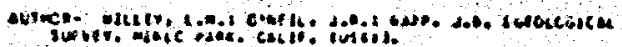

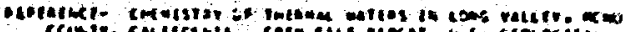

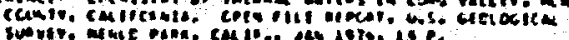

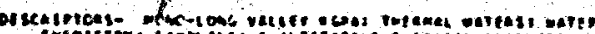

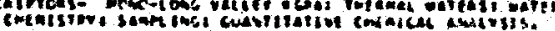

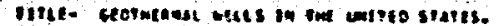

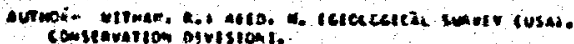

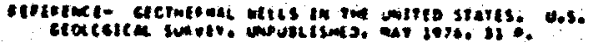

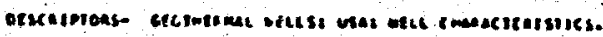

$$
\therefore
$$

$\operatorname{sen} n$

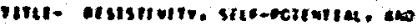

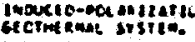

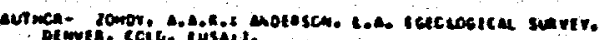

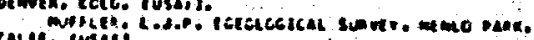
f caste. cussigi.

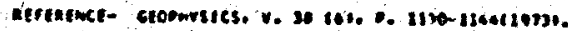

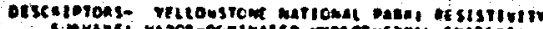

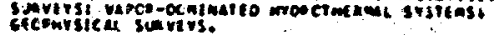
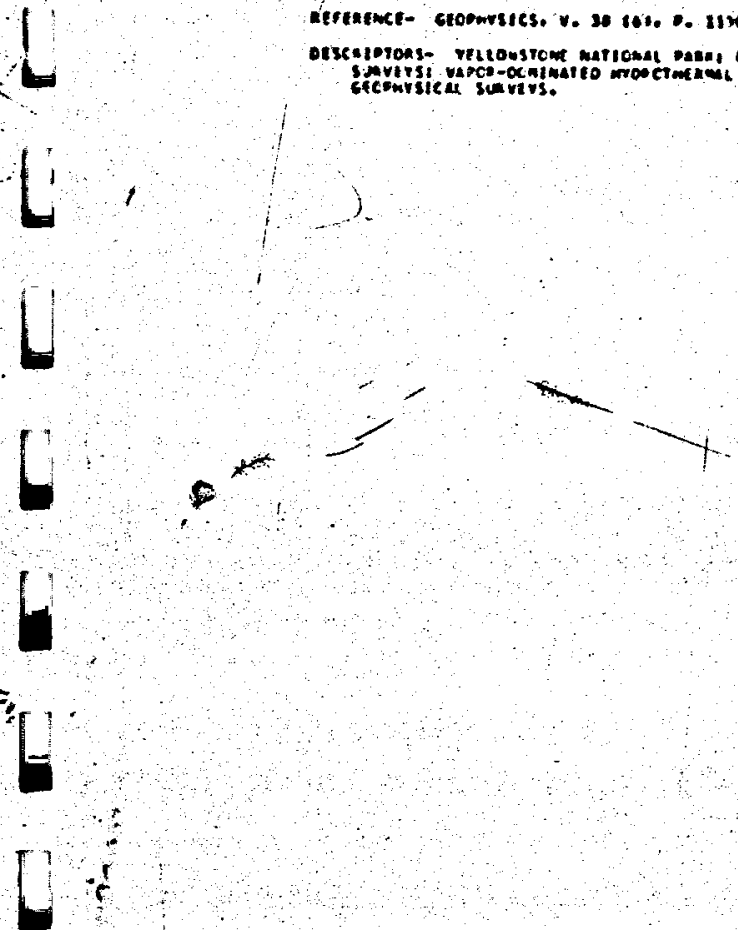